\title{
Rehabilitation of patients with a moderately to severely affected arm-hand in the sub-acute phase after stroke
}

Citation for published version (APA):

Franck, J. A. (2020). Rehabilitation of patients with a moderately to severely affected arm-hand in the subacute phase after stroke. [Doctoral Thesis, Maastricht University]. ProefschriftMaken Maastricht. https://doi.org/10.26481/dis.20200305jf

Document status and date:

Published: 01/01/2020

DOI:

10.26481/dis.20200305jf

Document Version:

Publisher's PDF, also known as Version of record

Please check the document version of this publication:

- A submitted manuscript is the version of the article upon submission and before peer-review. There can be important differences between the submitted version and the official published version of record.

People interested in the research are advised to contact the author for the final version of the publication, or visit the DOI to the publisher's website.

- The final author version and the galley proof are versions of the publication after peer review.

- The final published version features the final layout of the paper including the volume, issue and page numbers.

Link to publication

\footnotetext{
General rights rights.

- You may freely distribute the URL identifying the publication in the public portal. please follow below link for the End User Agreement:

www.umlib.nl/taverne-license

Take down policy

If you believe that this document breaches copyright please contact us at:

repository@maastrichtuniversity.nl

providing details and we will investigate your claim.
}

Copyright and moral rights for the publications made accessible in the public portal are retained by the authors and/or other copyright owners and it is a condition of accessing publications that users recognise and abide by the legal requirements associated with these

- Users may download and print one copy of any publication from the public portal for the purpose of private study or research.

- You may not further distribute the material or use it for any profit-making activity or commercial gain

If the publication is distributed under the terms of Article $25 \mathrm{fa}$ of the Dutch Copyright Act, indicated by the "Taverne" license above, 


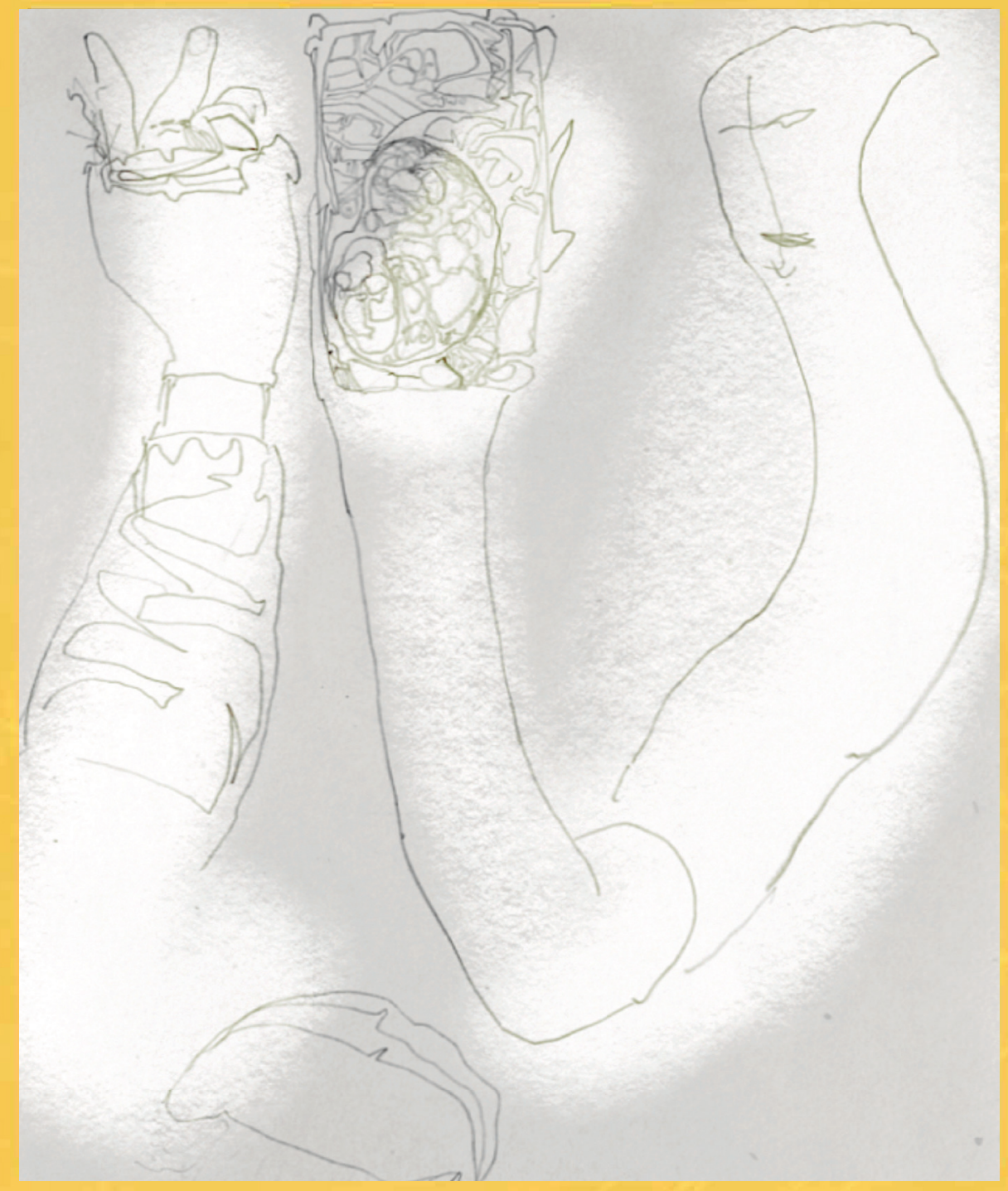

REHABILITATION OF PATIENTS WITH A MODERATELY TO SEVERELY AFFECTED ARM-HAND IN THE SUB-ACUTE PHASE AFTER STROKE

Johan Anton Franck 

Rehabilitation of patients with a moderately to severely affected arm-hand in the sub-acute phase after stroke

Johan Anton Franck 
(C) copyright Johan Anton Franck, Maastricht 2020

Cover design: Joop Noordkamp

Printing: ProefschriftMaken || www.proefschriftmaken.nl

ISBN 9789463807029

All rights reserved. No part of this publication may be reproduced, stored in a retrieval system or transmitted, in any form or by any means, electronic, mechanical, photocopying, recording or otherwise, without prior permission of the author or the copyright-owning journals for previous published chapters. 


\title{
Rehabilitation of patients with a moderately to severely affected arm-hand in the sub-acute phase after stroke
}

\author{
Proefschrift \\ Ter verkrijging van de graad van doctor \\ aan de Universiteit Maastricht, \\ op gezag van de Rector Magnificus, \\ Prof. dr. Rianne M. Letschert, \\ Volgens het besluit van het College van Decanen, \\ In het openbaar te verdedigen \\ op donderdag 5 maart 2020 om 16.00 uur \\ door \\ Johan Anton Franck
}




\section{Promotor}

Prof. dr. R.J.E.M. Smeets

\section{Copromotor}

Dr. H.A.M. Seelen

\section{Beoordelingscommissie}

Prof. dr. R.J. van Oostenbrugge (voorzitter)

Prof. dr. ir. J.H Buurke (University of Twente)

Prof. dr. A.M.C.F. Verbunt (Maastricht University)

Prof. dr. A.C.H. Geurts (Radboud University Medical Centre)

Prof. dr. A.T. Sack (Maastricht University) 


\section{Table of Contents}

\section{Chapter 1}

General Introduction

\section{Chapter 2}

Concise Arm and hand Rehabilitation Approach in Stroke (CARAS): A practical and evidence-based framework for clinical rehabilitation management

\section{Chapter 3}

Changes in arm-hand function and arm-hand skill performance in patients after stroke during and after rehabilitation

\section{Chapter 4}

Changes in actual arm-hand use in stroke patients during and after clinical rehabilitation involving a well-defined arm-hand rehabilitation program: A prospective cohort study

\section{Chapter 5}

Added-value of early post-stroke spasticity reduction during arm-hand rehabilitation in improving functional arm-hand skill performance: A multiple baseline single case experimental design study

\section{Chapter 6}

Added-value of spasticity reduction to improve arm-hand skill performance in sub-acute stroke patients with a moderately to severely affected arm-hand

\section{Chapter 7}

Evaluation of a functional hand orthosis combined with electrical stimulation adjunct to arm-hand rehabilitation in sub-acute stroke patients with a severely to moderately affected hand function

\section{Chapter 8}

Effects of a dynamic hand orthosis for functional use of the impaired upper limb in sub-acute stroke patients: A multiple single case experimental design study

\section{Chapter 9}

General discussion

\section{Chapter 10}

Valorization 
Summary

Samenvatting

299

Dankwoord

309

About the author

315 


\section{Chapter}

Rehabilitation of patients with a moderately to severely affected arm-hand in the sub-acute phase after stroke 

Stroke, also known as cerebrovascular accident (CVA) is the sudden death of brain cells due to lack of oxygen caused by blockage of blood flow or rupture of an artery to the brain [1]. There a two types of stroke, ischemic and hemorrhagic. In ischemic stroke, severe disturbance of the blood supply to brain tissue leads to a deprivation of oxygen supply to brain tissue and causes neuronal death [2]. A hemorrhagic stroke is either a brain aneurism burst or a weakened blood vessel leak. The bleeding may cause lesions to migrate and dissect through less-dense white matter and into the ventricles, causing swelling and (intracranial) pressure, damaging cells and brain tissue [3].

Worldwide, stroke is the second leading cause of death and the third leading cause of disability [4]. Fifteen million people suffer a stroke each year and almost 6 million people die from it. During the last four decades, stroke incidence declined by $42 \%$ in high-income countries [5], likely because of the increased use of specific prevention medication such as statins and anti-hypertensives [6]. During this time period, in lowincome countries stroke however, incidence has more than doubled [5]. A further increase in the global burden of stroke is expected, mainly due to the increase in disability-adjusted life years in developed countries and the ageing population [7, 8]. In addition, compared to high-income countries, stroke, on average, occurs 15 years earlier in low-and middle income countries and affects individuals at the peak of their productive life, thus creating an enormous impact on countries' socio-economic development [1]. In the Netherlands, in 2017, stroke incidence (excluding TIA (transient ischemic Attack)) amounted to 38,800 , whereas stroke year prevalence was 341,100 and mortality was 9,180 . In 2011 , in the Netherlands, costs related to care for patients suffering from a stroke amounted to almost 2.3 billion Euro [9].

The most common and widely recognized impairment caused by stroke is motor impairment, often stated as a loss or limitation in mobility typically affecting movement in the face, arm and leg of one side of the body [10,11]. Loss of movement in the arm and hand is very common after stroke and is often persistent and disabling [12]. About $33 \%$ to $66 \%$ of the stroke survivors are left with a severely impaired, non-functional arm-hand [13], and, depending on the outcome measures used, only $5 \%$ to $20 \%$ achieve full recovery of arm-hand function at 6 months [13]. Patients, after stroke, rate 
improving their arm-hand function and incorporating their paretic hand in daily activities as a top priority in their rehabilitation [14-16].

Even when stroke survivors experience some return of arm-hand function, it remains a common and significant source of long-term disability [17]. Symptoms like loss of strength and sensation, pain and spasticity in the affected arm-hand often lead to numerous restrictions in the daily lives of those affected. Their capacity to perform basic self-care and household activities and their ability to regain self-confidence in participation and taking up societal roles are reduced. Ultimately, this has a negative impact on the patient's emotional and psychological well-being [18, 19], and is associated with a poor perception of health-related quality of life [20].

Arm-hand rehabilitation in the post-stroke phase is continuously subject to changes and developments from both a research and a clinical perspective [21, 22]. Whereas acute treatment options after hemorrhagic stroke remain limited [23], in ischemic brain injuries acute stroke therapies such as reperfusion or neuroprotection have been developed to limit the severity of the injury [24]. Both existing and novel rehabilitation treatments need to take these new developments into account. As rehabilitation treatments aim to maximize function in brain areas that survived the stroke, or provide compensatory approaches to improve overall function [21], here at least two important domains regarding arm-hand recovery and arm-hand interventions in the post-stroke phase can be identified, i.e.:

1. Characterization of arm-hand recovery processes in association with poststroke brain plasticity, outcomes of which may support stratification and selection of those patients who are likely to positively respond to certain armhand rehabilitation programs [25], and

2. Development of novel interventions aimed at improving arm-hand performance during rehabilitation [7].

Many rehabilitation interventions have been developed over the last decades, aimed at maximizing arm-hand recovery in order to improve functional ability and quality of life [11, 26-28]. These interventions cover a wide range of different exercises or training methods, the use of equipment or techniques, and/or pharmacological interventions 
targeting neural mechanisms in order to enhance motor recovery [29]. This myriad of possibilities, in turn, makes it difficult for clinicians to decide on the most appropriate (combination of) therapy elements. In order to aid clinicians in their choices during clinical decision making, several authors have provided extensive overviews with respect to rehabilitation techniques and interventions aimed at arm-hand improvement [30-35]. Yet another difficulty for clinicians lies in the fact that, although arm-hand treatment delivered in stroke rehabilitation settings normally consist of a complex array of interventions, adjusted to the patient's individual needs, the set-up of most evaluation studies with respect to arm-hand interventions, reported in literature, deviates from these real-life arm-hand treatment circumstances. For example, the majority of the evaluation studies consist of a single intervention. Often, such intervention is firmly protocol-based to serve a narrow band of well-described patients, and is less adaptable to accommodate patients with different levels of impairment after stroke or patients with specific needs [37, 38] as commonly seen in daily clinical practice. A third source of difficulty for clinicians concerns the fact that, although it is generally acknowledged that task-specific training should be at the heart of treatment, it is still unclear how task-related interventions should be tailored to (reduce the consequences of) the specific underlying impairments.

\section{Arm - hand rehabilitation in sub-acute stroke patients with a moderately to severely affected arm-hand}

Whereas in patients with a mildly affected arm-hand improvements in arm-hand function (AHF) and arm-hand skill performance (AHSP) occur more quickly, the relearning of motor abilities during arm-hand rehabilitation for stroke patients with a moderately to severely affected arm-hand is slower, or even absent.

In $50 \%$ of patients with a severe paresis no recovery of the affected arm and hand is observed [39], due to the absence in the ipsilesional corticomotor excitability [25]. Immediately after admission to rehabilitation, these patients often display a combination of no hand function and a low motor ability level due to severe muscle weakness [40], while their prospect regarding functional recovery is limited [41, 42]. 
Stroke patients with a moderate to severely affected arm hand often experience comorbidities like cognitive deficits [43], depression [44], sensomotor deficits [45], shoulder pain [46, 47], spasticity [48] or edema [49] which even further hamper progressions in AHF and AHSP. Also, experiencing serious arm-hand capacity problems may have a negative impact on a patient's sense of self-efficacy. A low level of self-efficacy may lead to diminished patient's beliefs and self-perceived performance which, in turn, is associated with sub-optimal use of the affected arm-hand, thus leading to a vicious circle. Given the above, to both regain and maintain AHF and AHSP in patients with a moderately to severely affected hand is challenging.

In order to re-use the affected arm-hand in task-performance situations, a stepwise clinical management procedure must be developed to identify the nature of interacting constraints (among others motor control problems) that influence skill acquisition in the affected arm-hand [51]. Subsequently, a treatment plan must be developed in order to enable the patient to choose the most efficient strategy towards successful taskperformance.

A small change in AHF may represent a clinically meaningful change in AHSP and/or arm-hand performance. For example: being able to use the affected arm-hand for simple passive and active stabilization tasks leads to the patient being able to perform bimanual activities, like fixating bread while making a sandwich. However, it is quite difficult to recognize (subtle) improvements in AHF and AHSP, to encourage moderately and severely affected stroke patients to push their boundaries, and to create perspectives for these patients to re-use their affected hand more quickly in daily tasks outside the rehabilitation setting $[52,53]$. For this reason, AHF and/or AHSP improvements gained during therapy must be used to stimulate the patients' beliefs about their (growing) ability to influence their level of arm-hand performance. The transferability of learned skills to other (non-trained) skills must be enhanced [54, 55] and optimal therapy conditions (i.e. sufficient resources, state-of-the-art equipment, therapy time and therapy intensity) must be provided [56].

The need for comprehensive arm-hand rehabilitation programs, featuring essential aspects like adequately well-described content, dose and duration, has been acknowledged [38, 58, 59]. These rehabilitation programs should accommodate a broad spectrum of hand severity levels, including patients with a moderately to severely affected arm-hand, typically seen in daily medical rehabilitation practice. Nevertheless, currently, the majority of interventions targeting the affected arm-hand are protocolled 
interventions targeting stroke patients with a mildly impaired arm-hand using strict inclusion criteria. For this reason, these treatment protocols are less applicable in heterogeneous patient populations as commonly seen in rehabilitation practice, also holding patients with a moderately to severely affected arm-hand. Taking into account the abovementioned factors, the decision-making process in order to select the most appropriate intervention for a particular patient is challenging for clinicians in day-today clinical practice.

To understand the way in which arm-hand rehabilitation influences the neurobiology of the central nervous system regarding therapy content, timing of treatment and intensity of treatment is of considerable importance [60]. Knowledge with respect to these aspects should be leading in the design of the most appropriate arm-hand rehabilitation strategy, fitting the needs of the individual patient. Another important aspect of armhand rehabilitation is to make patients recognise their improved performance, and experience successful task performance in daily activities. Recognizing improvements may encourage stroke patients to push their boundaries and create perspectives to start re-using their affected hand more quickly in daily tasks. Ultimately, patients facilitate themselves in creating an optimal retention of how to use the full potential of their affected hand by also using the affected hand outside therapeutic situations, i.e. in their own daily life pursuits.

\section{Changes in arm-hand function, arm-hand skill performance and actual arm-hand use during and after rehabilitation}

Applying assessments to determine the effectiveness of arm-hand rehabilitation treatments and to monitor the patients' progressions during or after rehabilitation are indispensable both in clinical practice and in research. The application of the International Classification of Functioning, Disability and Health (ICF) framework [61] is supportive in differentiating three levels of human functioning, i.e. function level (body structures and function), activity level (task execution) and participation level (taking up societal roles). The term Arm Hand Function (AHF), used throughout this thesis, refers to the ICF function level. Arm-Hand Skill Performance (AHSP) refers to 
the ICF activity level, which is sub-classified into capacity and performance [62]. Performance can be sub-classified into perceived performance and actual performance. Perceived performance concerns the level of manual skill ability as observed by therapists in patients with arm-hand impairments or as perceived by patients themselves in daily life situations. Actual performance reflects the patient's objectively detectable level of AHSP as performed in his or her current environment [62] and can be subdivided into, a) amount of arm-hand use, i.e. how frequent and/or how much the arm-hand is used, and b) the quality with which the arm-hand is used during tasks or skills [62]. The application of the ICF model in rehabilitation allows clinicians to gain insight into the specific functional profile of each patient. Being able to objectively quantify patient's performance at the level of function, activity and participation is essential in identifying the relationships between these three levels and to fine-tune each patient's individual arm-hand rehabilitation treatment [63].

To improve AHF and AHSP in a therapeutic environment is done in order to achieve actual use of the affected hand in daily life performance situations outside the rehabilitation setting. Actual use of the affected hand in daily life performance strongly depends on the severity of the arm-hand impairment in the initial phase post-stroke [31, 64, 65]. Furthermore, it is known that after having been discharged from rehabilitation, arm-hand capacity may deteriorate and actual arm-hand use of the paretic arm and hand in daily life may decrease $[34,66,67]$. Rand et al. reported that in patients with a moderately or severely affected hand, the intensity of arm-hand use on the affected side sharply dropped during the rehabilitation phase [68]. De Niet et al. and Michielsen et al. found that chronic stroke patients hardly use their affected armhand in daily performance $[69,70]$. The rationale behind these findings related to the limited and even decreased use of the affected arm-hand is still poorly understood. It neither well understood at what rate such deterioration (or improvement) occurs, nor in which patient categories, i.e. patients with a certain level of arm-hand severity, this is most prominent. Taub and colleagues found substantial improvements on real armhand use outside the clinical setting in chronic stroke patients with a mildly affected arm-hand when using a behavioural strategy in combination with an intensive armhand training paradigm [71].

On may assume that, in order to use the affected hand in performing daily activities after rehabilitation, patients need to achieve their maximum motor ability level during 
rehabilitation. However, as already reported by others, the motor ability levels as measured in the clinical setting are considered to be different from the actual real-world arm-hand use [72-74]. The lack of information about the proportional improvement or deterioration to be expected in stroke survivors in the sub-acute phase after stroke may lead to difficulties for clinicians to make decisions about arm-hand treatment objectives and concomitant prognostics regarding arm-hand skill performance and actual arm-hand use, in particular in patients with a moderately to severely affected hand. It would, however, be of great value to have an insight in a more detailed level of retention of training effects in the time after cessation of the training, in order to assess whether arm-hand performance has stabilized or maybe even improved beyond clinical discharge values. Clarification of these issues is essential in order to develop more effective arm-hand rehabilitation interventions that may augment and/or maintain arm-hand AHF, AHSP and actual arm-hand use levels in stroke patients with a moderately to severely affected arm-hand.

\section{Evaluation of technological assistive devices and pharmacological interventions adjunct to arm-hand rehabilitation therapy in sub-acute stroke patients with a moderately to severely affected arm-hand}

Both the use and the impact of technological and pharmacological interventions that may enhance functional recovery or restoration of arm-hand function and arm-hand skill performance are growing [75]. The majority of such interventions is designed in order to augment existing therapies delivered by clinicians.

With respect to arm-hand rehabilitation in post-stroke phase, modest, though significant results are found for robotic-assistive arm training on arm function, strength and activities of daily living [35]. Regarding pharmacological trials aimed at enhancing motor recovery, significant results have been found in the application of selective serotonin reuptake inhibitor (SSRI) and L-dopa [29]. However, pharmacological interventions like, for instance, the application of botulinum toxin targeting improvements on arm-hand use, have not yet been observed [77]. Nevertheless, even when new developments are proven effective, clinicians may still experience difficulties to implement such new interventions swiftly into current and future therapy regimes 
$[28,78]$. First, it is challenging to keep up with the latest evidence, especially given the speed of developments in e.g. technology [79, 80].

Secondly, clinicians may face organisational issues when trying to adopt and implement new insights into daily clinical practice. New insights and techniques are introduced into practice in order to optimize a clinical pathway. However, two aspects are important here. Firstly, it is important to determine the added-value of new developments before leaving conventional treatment concepts. Secondly, it is important to determine the level of efficacy of the conventional therapy performed before novel treatment elements are introduced. Data obtained from stroke patients who received a well-documented form of therapy-as-usual can then be used as reference.

Most arm-hand rehabilitation programs lack transparency, which makes it difficult to determine the actual status of the content and dose of arm-hand training interventions delivered and how this influences AHF, AHSP and actual arm-hand use in a heterogeneous population typically seen in stroke rehabilitation $[58,76]$. To contrast newly developed training interventions in the absence of a well-defined form of armhand training is difficult. It may hamper the evaluation of novel developments like, for instance, the use of technological assistive devices or pharmacological interventions aimed at AHF and AHSP improvement in rehabilitation.

A critical factor contributing to successful rehabilitation of the affected hand after stroke is the provision of sufficient training intensity $[52,56,81]$ within the limited time window of spontaneous recovery [82-84]. Patients with a moderately to severely affected armhand are often unable to generate sufficient voluntary force in order to execute grasp movements independently $[85,86]$. For this reason they are often not engaged in highintensive, task-oriented training programs within the critical window of opportunity [87], which may influence improvements in AHF and AHSP negatively, especially in the subacute phase.

Several technological and pharmacological interventions are available in order to facilitate grasp and release movements independently in moderately to severely affected stroke patients. Though, the application of these interventions in clinical practice is limited due to several barriers like costs, questionable superiority over standard care, and aspects of usability $[80,88]$. Translation of these interventions to 
the clinical setting may also be hampered by the manner in which the intervention has been evaluated. The majority of these interventions are investigated using strict methodological considerations and results are often expressed in group averages, making it more difficult to translate such results to specific individual cases.

To evaluate the added-value of technological and pharmacological interventions within the complex nature of arm-hand rehabilitation could lead to more generalizable and translatable findings for the practicing clinician to use.

In contrast to group-related research, single-case experimental designs (SCED) provide an evaluation method that may be more suitable in heterogeneous populations like sub-acute stroke patients with a moderately to severely affected hand [89]. The application of the SCED, in conjunction with a well-documented form of arm-hand therapy used as standardized rehabilitation approach to contrast novel interventions, may provide opportunities to systematically investigate such novel interventions.

\section{Aim of the thesis}

The overall aim of this thesis is to provide a perspective on clinical management of the moderately to severely affected arm-hand in a heterogeneous group of sub-acute stroke patients commonly seen in stroke rehabilitation.

This thesis is divided in three parts: The first part outlines the Concise Arm and hand Rehabilitation Approach in Stroke (CARAS), a practical and evidence-based framework developed to support clinicians to structure arm-hand rehabilitation interventions in post-stroke phase. The second part reports on a prospective cohort study into the systematic evaluation of the proportional improvement or deterioration in arm-hand performance during and after rehabilitation in a broad group of stroke survivors typically seen in the rehabilitation clinic, who participated to CARAS. The third part encompasses three systematic evaluations, two of which focus on the (functional) added value of dynamic orthoses, and one on the application of botulinum toxin, all adjunct to CARAS provided to patients with a moderately to severely affected arm-hand in the sub-acute phase after stroke. 


\section{Outline of the thesis}

Chapter 2 explains the development and rationale of CARAS. In chapter 3 we investigated differences in the rate of improvement or deterioration in arm-hand function, arm-hand capacity and self-perceived arm-hand skill performance, during the rehabilitation period and up till 12 months after patients' protocolled rehabilitation treatment. Furthermore, differentiations in arm-hand function, arm-hand capacity and self-perceived arm-hand skill performance between three subgroups of stroke patients, i.e. patients with either a severely, moderately or mildly affected functional arm-hand have been investigated. In line with chapter 3, chapter 4 reports on the actual use of the arm-hand during daily unimanual and bimanual tasks, measured using accelerometry and expressed as 'Intensity-of arm-hand-use' and 'Duration-of-armhand-use'. Actual use has been measured during the rehabilitation phase and until one year post-discharge in sub-acute stroke patients with a severely, moderately or mildly affected arm-hand. Chapter 5 outlines a design protocol comprising three methodological approaches in order to assess the rate of improvement in arm-hand performance that may be attributable to a spasticity reducing treatment relative to 'therapy as usual only' in sub-acute stroke patients. In Chapter 6 the added-value of early spasticity reduction to improve arm-hand skill performance in sub-acute stroke patients with a moderately to severely affected hand is evaluated.

Chapter 7 and chapter 8 describe the usability and the effectiveness of two dynamic hand orthoses, adjunct to therapy-as-usual, on functional use of the moderately/severely impaired hand in sub-acute stroke patients. Finally, Chapter 9 summarizes the results of the studies performed, and discusses clinical implications, methodological considerations and future perspectives in clinical practice and research. 


\section{References}

1. Johnson W, Onuma O, Owolabi M, Sachdev S. Stroke: a global response is needed. 2016; 21(94):[634-5 pp.].

2. Su H. Inflammation and genetic factors in stroke pathogenesis. Neurology Neuroimmunology \& Neuroinflammation. 2017;4:260-2. doi: 10.20517/2347-8659.2017.22.

3. Rymer M. Hemorrhagic Stroke: Intracerebral Hemorrhage. Missouri Medicine. 2011;(108):50-4.

4. WHO. Global Health Estimates. 2016.

5. Owolabi M, Akarolo-Anthony S, Akinyemi R, Arnett D, Gebregziabher M, Jenkins C. The burden of stroke in Africa: a glance at the present and a glimpse into the future. Cardiovasc J Afr. 2015;26(2):27-38. doi: org/10.5830/CVJA-2015-038. PubMed Central PMCID: PMC25962945.

6. Guzik A, Bushnell C. Stroke Epidemiology and Risk Factor Management. Cerebrovascular Disease. 2017;23(1):15-39. doi: doi:10.1212/CON.0000000000000416.

7. Bernhardt J, Hayward KS, Kwakkel G, Ward N, Wolf S, Borschmann K, et al. Agreed definitions and a shared vision for new standards in stroke recovery research: The Stroke Recovery and Rehabilitation Roundtable taskforce. international journal of Stroke. 2017;12(5):444-50. doi: 10.1177/1747493017711816.

8. Feigin VL, Krishnamurthi RV, Parmar P, Norrving B, Mensah GA, Bennett DA. Update on the global burden of ischemic and hemorrhagic stroke in 1990-2013. Neuroepidemiology. 2015;45(3):161-76. doi: 10.1159/000441085.

9. (RIVM). RvVeM. Stroke, Figures \& Context, Burden of Disease 2019. Available from: https://www.volksgezondheidenzorg.info/onderwerp/beroerte/cijfers-context

10. Wade DT. Evaluating outcome in stroke rehabilitation (quality control and clinical audit). Scand J Rehabil Med Suppl. 1992;26:97-104. PubMed PMID: 1488644.

11. Langhorne P, Coupar F, Pollock A. Motor recovery after stroke: a systematic review. Lancet Neurol. 2009;8:741-54.

12. Lai SM, Studenski S, Duncan PW, Perera S. Persisting consequences of stroke measured by the Stroke Impact Scale. Stroke; a journal of cerebral circulation. 2002;33(7):1840-4. PubMed PMID: 12105363.

13. Kwakkel G, Kollen BJ, van der Grond J, Prevo AJ. Probability of regaining dexterity in the flaccid upper limb: impact of severity of paresis and time since onset in acute stroke. Stroke; a journal of cerebral circulation. 2003;34(9):2181-6. PubMed PMID: 12907818.

14. Barker RN, Brauer SG. Upper limb recovery after stroke: the stroke survivors' perspective. Disability and rehabilitation. 2005;27(20):1213-23. Epub 2005/11/22. doi: 10.1080/09638280500075717. PubMed PMID: 16298923. 
15. Dobkin $\mathrm{BH}$. Clinical practice. Rehabilitation after stroke. $\mathrm{N}$ Engl $\mathrm{J}$ Med. 2005;352(16):1677-84.

16. Barker RN, Gill TJ, Brauer SG. Factors contributing to upper limb recovery after stroke: a survey of stroke survivors in Queensland Australia. Disability and rehabilitation. 2007;29(13):981-9.

17. Broeks JG, Lankhorst GJ, Rumping K, Prevo AJ. The long-term outcome of arm function after stroke: results of a follow-up study. Disability and rehabilitation. 1999;21(8):357-64. PubMed PMID: 10503976.

18. Keppel CC, Crowe SF. Changes to body image and self-esteem following stroke in young adults. Neuropsychol Rehabil. 2000;10:15-31.

19. Wyller TB, Sveen U, Sødring KM, Pettersen AM, Bautz-Holter E. Subjective well-being one year after stroke. Clinical rehabilitation. 1997;11(2):139 - 45.

20. Franceschini M, La Porta F, Agosti M, Massucci M. Is health-related-quality of life of stroke patients influenced by neurological impairments at one year after stroke? European journal of physical and rehabilitation medicine. 2010;46(3):389-99. Epub 2010/10/12. PubMed PMID: 20927005.

21. Cramer S, Wolf S, Adams H, Chen D, Dromerick A, Dunning K, et al. Stroke Recovery \& Rehabilitation Research: Issues, Opportunities, and the NIH StrokeNet. Stroke 2017;48(3):813-9. doi: 10.1161/STROKEAHA.116.015501.

22. Bernhardt J, Borschmann K, Boyd L. Moving rehabilitation research forward: developing consensus statements for rehabilitation and recovery research. International Journal of Stroke. 2016;11:454-8.

23. Adams HP, Nudo RJ. Management of patients with stroke: Is it time to expand treatment options? Ann Neurol. 2013;74:4-10.

24. Bhaskar S, Stanwell P, Cordato D, Attia J, Levi C. Reperfusion therapy in acute ischemic stroke: dawn of a new era? BMC Neurology. 2017;18(8):1-26.

25. Byblow W, Stinear C, Barber P, Petoe M, Ackerley S. Proportional Recovery After Stroke Depends on Corticomotor Integrity. ANN NEUROL 2015;78:848-59.

26. Johansson BB. Current trends in stroke rehabilitation. A review with focus on brain plasticity. Acta Neurologica Scandinavica. 2011;123(3):147-59. doi: 10.1111/j.16000404.2010.01417.

27. Arya KN, Pandian S, Verma R, Garg R. Movement therapy induced neural reorganization and motor recovery in stroke: a review. Journal of Bodywork and Movement Therapies. 2011;15(4):528-37. doi: 10.1016/j.jbmt.2011.01.023.

28. Brewer L, Horgan F, Hickey A, Williams D. Stroke rehabilitation: recent advances and future therapies. An International Journal of Medicine. 2013;106(1):11-25. doi: 10.1093/qjmed/hcs174. 
29. Cramer S. Drugs to Enhance Motor Recovery After Stroke. Stroke 2015;46(10):29983005. doi: 10.1161/STROKEAHA.115.007433.

30. Veerbeek JM, van Wegen E, van Peppen R, van der Wees PJ, Hendriks E, Rietberg M, et al. What is the evidence for physical therapy poststroke? A systematic review and metaanalysis. PloS one. 2014;9(2):e87987. Epub 2014/02/08. doi: 10.1371/journal.pone.0087987. PubMed PMID: 24505342; PubMed Central PMCID: PMCPMC3913786.

31. Pollock A, Farmer SE, Brady MC, Langhorne P, Mead GE, Mehrholz J, et al. Interventions for improving upper limb function after stroke. The Cochrane database of systematic reviews. 2014;11:CD010820. Epub 2014/11/12. doi: 10.1002/14651858.CD010820.pub2. PubMed PMID: 25387001.

32. French B, Thomas LH, Coupe J, McMahon NE, Connell L, Harrison J, et al. Repetitive task training for improving functional ability afterstroke (Review). Cochrane Database of Systematic Reviews. 2016;(11). doi: 10.1002/14651858.CD006073.pub3.

33. Coupar F, Pollock A, Legg LA, Sackley C, van Vliet P. Home-based therapy programmes for upper limb functional recovery following stroke (Review). Cochrane Database of Systematic Reviews 2012;(5). doi: 10.1002/14651858.CD006755.pub2.

34. Hayward K, Barker R, Brauer S. Interventions to promote upper limb recovery in stroke survivors with severe paresis: a systematic review. Disability and rehabilitation. 2010;32(24):1973-86. PubMed PMID: 20964563.

35. Mehrholz J, Pohl M, Platz T, Kugler J, Elsner B. Electromechanical and robot-assisted armtraining for improving activities of daily living, armfunction, and arm muscle strength after stroke (Review). Cochrane Database of Systematic Reviews. 2018;(9). doi: 10.1002/14651858.CD006876.pub5.

36. Winstein C, Lewthwaite R, Blanton S, Wolf L, Wishart L. Infusing Motor Learning ResearchInto Neurorehabilitation Practice: A Historical PerspectiveWith Case Exemplar From the Accelerated Skill Acquisition Program. JNPT. 2014;38:190-200.

37. French B, Leathley M, Sutton C, McAdam J,Thomas L, Forster A, et al. A systematic review of repetitive functional task practice with modelling of resource use, costs and effectiveness. Health Technology Assessment 2008;12(30).

38. McDonnell M, Hillier S, Esterman A. Standardizing the Approach to Evidence-Based Upper Limb Rehabilitation after Stroke. Topics in stroke rehabilitation. 2013;20(5):432-40.

39. Zarahn E, Alon L, Ryan S, Lazar RM, Vry MS, Weiller C, et al. Prediction of motor recovery using initial impairment and fMRI 48 h poststroke. Cereb Cortex. 2011;21(12):2712-21.

40. Francis HP, Wade DT, Turner-Stokes L, Kingswell RS, Dott CS, Coxon EA. Does reducing spasticity translate into functional benefit? An exploratory meta-analysis. Journal of Neurology Neurosurgery Psychiatry. 2004;75:1547 - 51. 
41. Winters C, Kwakkel G, Nijland R, van Wegen E. When Does Return of Voluntary Finger Extension Occur Post-Stroke? A Prospective Cohort Study. PloS one. 2016;11(8):1-12. doi: 10.1371/journal.pone.0160528.

42. Stinear CM, Byblow WD, Ward SH. An update on predicting motor recovery after stroke. Annals of physical and rehabilitation medicine. 2014;57(8):489-98. Epub 2014/09/10. doi: 10.1016/j.rehab.2014.08.006. PubMed PMID: 25200094.

43. Mullick AA, Subramanian SK, Levin MF. Emerging evidence of the association between cognitive deficits and arm motor recovery after stroke: A meta-analysis. restorative Neurology and Neuroscience. 2015;33(3):389 - 403.

44. Kong K, Loh Y, Thia E, Chai E, Yin C. The Relationship between Poststroke Depression and Upper Limb Recovery in Patients Admitted to a Rehabilitation Unit Physical medicine and Rehabilitation. 2017;1(1):1-7.

45. Tyson SF, Hanley M, Chillala J, Selley AB, Tallis R. Sensory Loss in Hospital-Admitted People with Stroke: Characteristics, Associated Factors, and Relationship with Function. Neural Rehabilitation and Neural Repair. 2008;22(2):166-72. doi: 10.1177/1545968307305523.

46. Lindgren I, Jonsson AC, Norrving B, Lindgren A. Shoulder pain after stroke: a prospective population-based study. Stroke; a journal of cerebral circulation. 2007;38(2):343-8.

47. Ratnasabapathy Y, Broad J, Baskett J, Pledgern M, Marshallm J, Bonita R. Shoulder pain in people with a stroke: a population-based study. Clinical rehabilitation. 2003;17:304-11.

48. Wissel J, Verrier M, Simpson D, Charles D, Guinto P, Papapetropoulos S, et al. Poststroke spasticity: predictors of early development and considerations for therapeutic intervention. Physical medicine and Rehabilitation. 2015;7:60 - 7.

49. Boomkamp-Koppen HG, Visser-Meily JM, Post MW, Prevo AJ. poststroke hand swelling and oedema: prevalence and relationship with impairment and disability. Clinical rehabilitation. 2005;19(5):552-9.

50. Nijland RH, van Wegen EE, Harmeling-van der Wel BC, Kwakkel G. Presence of finger extension and shoulder abduction within 72 hours after stroke predicts functional recovery: early prediction of functional outcome after stroke: the EPOS cohort study. Stroke; a journal of cerebral circulation. 2010;41(4):745-50. Epub 2010/02/20. doi: 10.1161/strokeaha.109.572065. PubMed PMID: 20167916.

51. Davids K. The constraints- based approach to motor learning: implications for a non- linear pedagogy in sport and physical education. In: Ian Renshaw KD, Geert J.P. Savelsbergh, editor. Motor Learning in Practice: A Constraints-Led Approach. 1th. London: Routledge; 2010. p. 272.

52. Daly J, McCabe J, Holcomb H, Monkiewicz M, Gansen J, Pundik S. Long-Dose Intensive Therapy Is Necessary for Strong, Clinically Significant, Upper Limb Functional Gains and 
Retained Gains in Severe/Moderate Chronic Stroke. Neurorehabilitation and neural repair. 2019. doi: $10.1177 / 1545968319846120$.

53. Hayward KS, Kuys SS, Barker RN, Brauer SG. Can stroke survivors with severe upper arm disability achieve a clinically important change in arm function during inpatient rehabilitation? A multicentre, prospective, observational study. NeuroRehabilitation. 2014;35(1):17-23. Epub 2014/07/06. doi: 10.3233/nre-141096. PubMed PMID: 24990005.

54. Magill R. Transfer of Learning. Motor Learning and Control Concepts and Applications. Boston: Mc Graw Hill; 2007. p. 290-306.

55. Wulf $\mathrm{G}$, Shea C, Lewthwaite R. Motor skill learning and performance: a review of influential factors. Med Educ. 2010;44(1):75-84. PubMed PMID: 20078758.

56. Hayward KS, Brauer SG. Dose of arm activity training during acute and subacute rehabilitation post stroke: a systematic review of the literature. Clinical rehabilitation. 2015;29(12):1234 - 43.

57. Winters C, van Wegen EE, Daffertshofer A, Kwakkel G. Generalizability of the Proportional Recovery Model for the Upper Extremity After an Ischemic Stroke. Neurorehabilitation and neural repair. 2015;29(7):614-22. Epub 2014/12/17. doi: 10.1177/1545968314562115. PubMed PMID: 25505223.

58. Demetrios M, Gorelik A, Louie J, Brand C, Baquley IJ, Khan F. Outcomes of ambulantory rehabilitation programmes following botulinum toxin for spasticity in adults with stroke. $J$ Rehabil Med. 2014;46:730-7.

59. Ward NS, Brander F, Kelly K. Intensive upper limb neurorehabilitation in chronic stroke: outcomes from the Queen Square programme. cerebrovasc Dis. 2019;90:498-506. doi: 10.1136/jnnp-2018-319954.

60. Wahl A, Schwab M. Finding an optimal rehabilitation paradigm after stroke: enhancing fiber growth and training of the brain at the right moment. Frontiers in human neuroscience. 2014;8:1-12. doi: 10.3389/fnhum.2014.00381.

61. World Health Organization. International Classification of Functioning, Disability and Health: ICF. Geneva: World Health Organization, 2001.

62. Lemmens RJ, Timmermans AA, Janssen-Potten YJ, Smeets RJ, Seelen HA. Valid and reliable instruments for arm-hand assessment at ICF activity level in persons with hemiplegia: a systematic review. BMC Neurol. 2012;12:21. Epub 2012/04/14. doi: 10.1186/1471-2377-12-21. PubMed PMID: 22498041; PubMed Central PMCID: PMCРMC3352056.

63. Silva S, Corrêa F, Coelho de Morais Faria C, Buchalla M, Silva P, Corrêa J. Evaluation of post-stroke functionality based on the International Classification of Functioning, Disability, and Health: a proposal for use of assessment tools. Journal of Physical Therapy Science 2015;27:1665-70. 
64. Sveen U, Bautz-Holter E, Sodring KM, Wyller TB, Laake K. Association between impairments, self-care ability and social activities 1 year after stroke. Disability and rehabilitation. 1999;21(8):372 - 7.

65. Kwakkel G. Impact of intensity of practice after stroke: issues for consideration. Disability and rehabilitation. 2006;28(13-14):823-30. PubMed PMID: 16777769.

66. Gebruers N, Vanroy C, Truijen S, Engelborghs S, De Deyn PP. Monitoring of physical activity after stroke: a systematic review of accelerometry-based measures.

Archives of physical medicine and rehabilitation. 2010;91:288-97.

67. Taub E, Uswatte G, Mark VW, Morris DM. The learned nonuse phenomenon: implications for rehabilitation. Eura Medicophys. 2006;42(3):241-56. PubMed PMID: 17039223.

68. Rand D, Eng JJ. Disparity between functional recovery and daily use of the upper and lower extremities during subacute stroke rehabilitation. Neurorehabil Neural Repair. 2012;26:76-84.

69. Niet M, Bussmann JB, Ribbers GM, Stam H.The Stroke Upper-Limb Activity Monitor: Its Sensitivity to Measure Hemiplegic Upper-Limb Activity During Daily Life. Archives of physical medicine and rehabilitation. 2007;88:1121 - 6 .

70. Michielsen ME, Selles RW, Stam HJ, Ribbers GM, Bussmann JB. Quantifying nonuse in chronic stroke patients: a study into paretic, nonparetic and bimanual upper limb use in daily life. Archives of physical medicine and rehabilitation. 2012;93:1975-81.

71. Taub E, Uswatte G. Constraint-Induced Movement Therapy: A Family of Neurorehabilitation Treatments that Harnesses the Plasticity of the Central Nervous System. Neurol Rehabil 2013;19(3):161-75.

72. Thrane G, Emaus N, Askim T, Anke A. Arm use in patients with subacute stroke monitored by accelerometry: association with motor impairment and influence on self-dependence. J Rehabil Med. 2011;43:299-304.

73. Uswatte G, Foo WL, Olmstead H, Lopez K, Holand A, Simms LB. Ambulatory monitoring of arm movement using accelerometry: an objective measure of upper-extremity rehabilitation in persons with chronic stroke. Archives of physical medicine and rehabilitation. 2005;86(7):1498-501. PubMed PMID: 16003690.

74. Lang CE, Wagner JM, Edwards DF, Dromerick AW. Upper extremity use in people with hemiparesis in the first few weeks after stroke. Journal of Neurologic Physical Therapy. 2007;31:56-63.

75. Dietz V, Ward NS. Oxford Textbook of Neurorehabilitation.Oxford:Oxford university Press; 2015.

76. Rymer W, Jayaraman A. Promises and challenges of neurorehabilitation technology. In: Christopher K, editor. Oxford Textbook of Neurorehabilitation.Oxford:Oxford University Press; 2015. 
77. Andringa A, Port I, van Wegen E, Ket J, Meskers C, Kwakkel G. Effectiveness of Botulinum Toxin Treatment for Upper Limb Spasticity Poststroke Over Different ICF Domains: a Systematic Review and Meta-Analysis. Archives of physical medicine and rehabilitation. 2019. doi: doi.org/10.1016/j.apmr.2019.01.016.

78. Langhorne P, Bernhardt J, Kwakkel G. Stroke rehabilitation. Lancet. 2011;377:1693 - 702.

79. Herzlinger RE. Why innovation in health care is so hard. Harv Bud Rev. 2006;84(5):58-66.

80. Hughes AM, Burridge JH, Demain SH, Ellis-Hill C, Meagher C, Tedesco-Triccas L, et al. Translation of evidence-based Assistive Technologies into stroke rehabilitation: users' perceptions of the barriers and opportunities. BMC health services research. 2014;14:124. Epub 2014/03/14. doi: 10.1186/1472-6963-14-124. PubMed PMID: 24620739; PubMed Central PMCID: PMCPMC4007558.

81. Bell A, Wolke M, Ortez R, Jones T, Kerr A. Training intensity impacts motor rehabilitation efficacy following unilateral ischemic insult of the sensorimotor cortex in C57BL/6 mice. Neurorehabilitation and neural repair. 2014;29(6):590-8. doi: 10.1177/1545968314553031.

82. Buma F, Kwakkel G, Ramsey N. Understanding upper limb recovery after stroke. Restorative Neurology and Neuroscience. 2013;31:707 - 22.

83. Levin MF, Kleim JA, Wolf SL. What do motor "recovery" and "compensation" mean in patients following stroke? Neurorehabilitation and neural repair. 2009;23(4):313-9. PubMed PMID: 19118128.

84. Zeiler S, Krakauer J. The interaction between training and plasticity in the poststroke brain. Current opinion in neurology. 2013;26(6):609-16. doi: 10.1097/WCO.0000000000000025.

85. Canning CG, Ada L, Adams R, O'Dwyer NJ. Loss of strength contributes more to physical disability after stroke than loss of dexterity. Clinical rehabilitation. 2004;18(3):300 - 8.

86. Taub E, Miller NE, Novack TA, Cook EW 3rd, Fleming WC, Nepomuceno CS, et al. Technique to improve chronic motor deficit after stroke. Archives of physical medicine and rehabilitation. 1993;74(4):347-54. Epub 1993/04/01. PubMed PMID: 8466415.

87. Chae J. A critical review of neuromuscular electrical stimulation for treatment of motor dysfunction in hemiplegia. Assist Technol 2000;12(1):33-49.

88. Harvey RL. NeuroRehabilitation: New concepts in stroke rehabilitation. NeuroRehabilitation. 2018;43:1-2.

89. Tate RL, Rosenkoetter U, Wakim D, Sigmundsdottir L, Doubledaty J, Togher L. The Risk of Bias in N-of-1 Trials (RoBiNT) Scale: An expanded manual for the critical appraisal of single-case reports. Syndey 2015. 
CHAPTER 1 


\section{Chapter}

Concise Arm and hand Rehabilitation Approach in Stroke (CARAS): A practical and evidence-based framework for clinical rehabilitation management

Johan Anton Franck, Jos H.G. Halfens, Rob Johannes Elise Marie Smeets,

Henk Alexander Maria Seelen 


\section{Abstract}

The volume of information on new treatment techniques supporting the restoration of arm-hand function (AHF) and arm-hand skill performance (ASHP) in stroke survivors overwhelms therapists in everyday clinical practice when choosing the appropriate therapy.

The Concise Arm and hand Rehabilitation Approach in Stroke (CARAS) is designed for paramedical staff to structure and implement training of AHF and AHSP in stroke survivors.

CARAS is based on four constructs: a) stratification according to the severity of armhand impairment (using the Utrecht Arm-hand Test (UAT), b) the individual's rehabilitation goals and concomitant potential rehabilitation outcomes, c) principles of self-efficacy, and d) possibilities to systematically incorporate (new) technology and new evidence-based training elements swiftly.

The framework encompasses three programs aimed at treating either the severely (UAT 0-1), moderately (UAT 2-3) or mildly (UAT 4-7) impaired arm-hand. Program themes are: 'taking care of the limb and prevention of complications' (program 1), 'taskoriented gross motor grip performance' (program 2) and 'functional AHSP training' (program 3).

Each program is preceded and followed by an assessment. Training modularity facilitates rapid interchange/adaptation of sub-elements. Proof-of-principle in clinical rehabilitation has been established. CARAS facilitates rapid structured design and provision of state-of-the-art AHF and AHSP treatment in stroke patients.

Keywords: arm, hand, self-efficacy, stroke, therapy, task-oriented training

Running title: Concise arm-hand rehabilitation approach in stroke 


\section{Introduction}

Approximately $50 \%$ of stroke survivors experience unilateral motor deficit that leads to chronic upper extremity impairment. This results in limited functional use of the affected arm as well as reduced engagement in community life [1-5] and a poorer quality of life overall [6]. Four years after stroke, $67 \%$ of stroke survivors with initial unilateral motor deficit still experience nonuse or disuse of the affected arm as a major problem [1].

Motor rehabilitation aimed at arm-hand performance after stroke has changed substantially over the last decades. Previously, treatment mainly targeted the International Classification of Functioning, Disability and Health (ICF) function level [7]. Researchers now focus instead on ICF activity and participation level.

Well-explored training approaches have emerged [8-11] that address paresis and impaired motor control [12-14]. These approaches feature training elements such as meaningfulness; challenge; specificity; feasibility; and, when some arm-hand dexterity emerges, task-oriented and high-intensity training [15]. Further, these treatment programs include a wide variety of exercises that stroke survivors may use in therapeutic and/or home-based situations [16-21].

Task-oriented training [22-24] and constraint-induced movement therapy [25] focus on both the ICF activity level and participation level. In task-oriented approaches, patients are trained in specific functional, skill related tasks, preferably using real-life objects [26], thereby teaching patients to solve specific problems related to issues such as anticipatory locomotor adjustments or cognitive processing by using efficient goaloriented movement strategies [27, 28]. The positive transfer of learned skills to other (non-trained) skills occurs when similarities with the learned skill are present [29]. Functional treatment outcome in task-oriented training approaches is higher than in muscle strength training [15].

The increasing amount of evidence and studies related to arm-hand performance after stroke creates a new problem for modern day therapists treating stroke survivors. The sheer volume of information on new treatment techniques and technologies that could enhance functional recovery or restoration of arm-hand function and arm-hand skill performance may overwhelm therapists in day-to-day clinical practice when they 
have to choose the appropriate therapy for a patient. This potentially leads to the implementation of a patchwork of training regimens.

Translating the latest scientific evidence and results from clinical trials into clinically useful treatments is difficult [30-32], and although formal (national) guidelines for training exist, these recommendations cannot always keep up with the latest evidence, especially given the speed of technological developments [33].

In order to guide therapists in systematically designing a stroke patient's arm-hand rehabilitation program, the authors address four issues:

- The heterogeneity of the patient population and the associated patterns and levels of recovery of arm-hand skill performance [34, 35].

- The lack of adequate description and adaptation of treatment protocols for stroke survivors experiencing a broad variety of problems in daily life related to an impaired arm-hand.

- The encouragement of patients' beliefs about their ability to influence their level of performance, thus enabling them to train at and maintain a certain skill level. This makes the patient the main stakeholder in his or her training [36-38].

- The difficulty of swiftly implementing new insights into current and future therapy regimes $[9,11]$.

Related to these four issues, four potential solutions are proposed:

a) The presence or absence of dexterity in the affected arm-hand is the most important variable. When selecting the potentially most effective treatment, the recommendation is to stratify persons with an impaired arm-hand into a limited number of dexterity levels [11,35].

b) A well-described program offering stepwise, comprehensible procedures may facilitate transparency and could lead to outcomes that are more predictable. A (modular) program should span the full range of arm-hand impairments and related functional problems experienced, from 'taking care and prevention' to 'high intensity task-oriented training'. 
c) The patient's lack of engagement with arm-hand treatment may be overcome by using self-efficacy principles, which could also facilitate optimal transfer and retention of learning.

d) To allow for quick adaptations to novel and effective innovations, the content of training should be based on simple, easy-to-replace schedules organized into time blocks. When necessary, other training methods can replace these schedules' content without having to rearrange treatment planning.

The aim of this paper is to present the Concise Arm and hand Rehabilitation Approach in Stroke (CARAS) that therapists can use to structure and implement treatment of arm-hand function and arm-hand skill performance in stroke survivors based on a) level of arm-hand impairment, b) detailed training descriptions, c) principles of selfefficacy, and d) swift implementation of interventions.

\section{A: Level of arm-hand impairment}

CARAS encompasses three modular, group-based training programs divided into two parts, namely 'taking care and prevention' (Part 1) and 'high intensity, task-oriented arm-hand performance training' (Part 2; see Figure 1). Based on the Utrecht Arm-hand Test (UAT) scores [39], patients enrol in one of the training programs, each of which consists of well-described and time-delimited building blocks. 


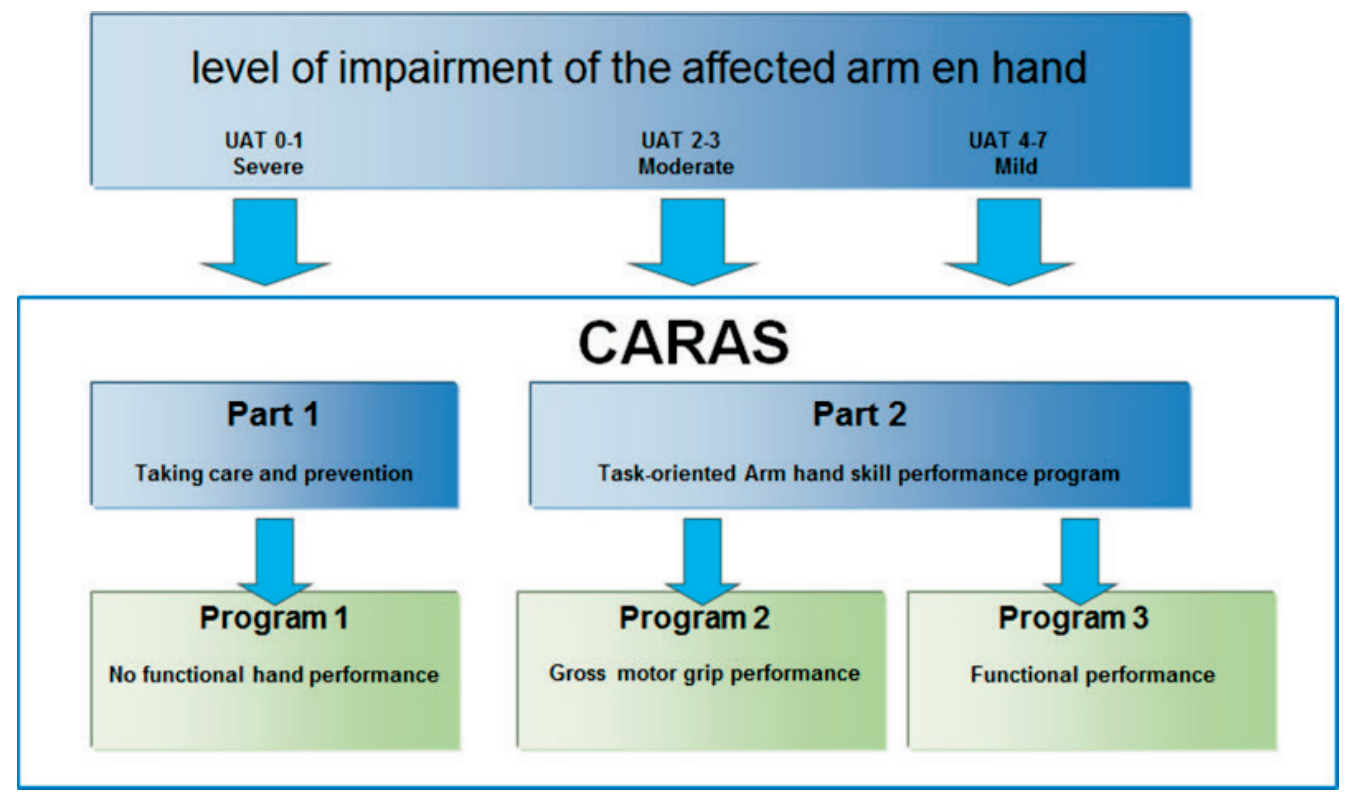

Figure 1. Schematic representation of CARAS and its constituent programs. UAT = Utrechtse Arm-hand Test

Part 1, encompassing program 1, is designed for stroke survivors who, due to the severity of the stroke, are not able to use their affected arm-hand for skill performance in daily life situations (non-functional arm-hand) because of inactivity, spasticity and/or stiffness. Eventually, this disuse can lead to secondary complications, such as pain, problems in performing basic activities of daily living, and hygienic issues [8, 40]. Therapists can manage these complications by coaching patients on how best to care for their impaired arm-hand.

Program 1 targets stroke survivors with an UAT score of 0-1. Interventions are directed towards enabling persons to keep the arm-hand in optimal condition, such as feeling comfortable in various postures both during resting and while performing daily life activities.

Part 2, encompassing programs 2 and 3, features high intensity, task-oriented armhand training in which patients learn to integrate their affected arm-hand into daily occupations to optimise their overall functional abilities in daily situations. In this part, a distinction is made between patients who have a moderately affected arm-hand (i.e. those who are able to use their affected arm-hand for passive and active stabilisation tasks like holding bread while making a sandwich) and patients with a mildly affected 
arm-hand (i.e. those who are able to use their affected arm-hand instantaneously in daily situations).

Program 2 targets stroke survivors with an UAT score of 2-3. These persons have to cope with a moderately impaired arm-hand and are able to use their affected hand to assist the non-paretic arm-hand during bimanual activities in daily life. This program is aimed at gross motor grip tasks, i.e. 'passive and active fixation' tasks, 'grasp and displace' tasks and simple bimanual daily life activities.

Program 3 targets stroke survivors with an UAT score of 4-7. These patients have the potential or are already able to spread the fingers and make isolated finger movements with the affected hand. From the perspective of motor learning, this allows them to use their arm-hand in functional tasks in daily life situations immediately from the start of rehabilitation. This program is aimed at 'grasp and displace' tasks, manipulation tasks and complex bimanual activities.

\section{B Training interventions}

Figure 2 shows the CARAS time schedule.

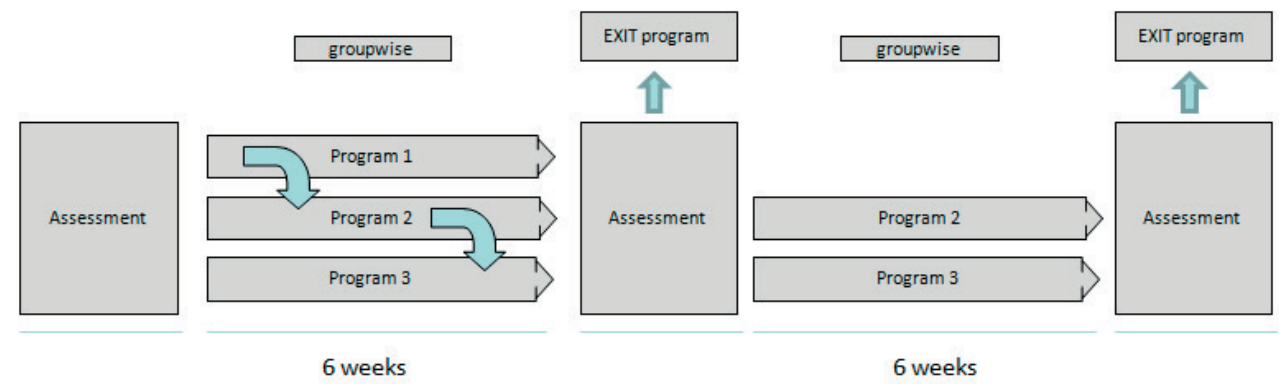

Figure 2. Time schedule of CARAS

In order to manage CARAS' group-based interventions adequately, groups should be limited to six patients. After establishing a baseline via an assessment, patients enrol 
in one of the three programs and start training for six consecutive weeks, followed by a second assessment. Progress is expressed in terms of functional goals reached, based on measures gauging performance levels (e.g. ABILHAND)[41] and capacity levels (e.g. Action Research Arm Test [42], or the Fugl-Meyer Motor Assessment [43]. Depending on these results, it is possible for the patient to choose a second 6-weeks period of training. Furthermore, depending on the progress made, a patient can switch from program 1 to program 2 when he or she has regained dexterity in the affected arm-hand (improving from UAT 1 to 2), or from program 2 to program 3 when he or she shows an increase in selectivity in the affected hand, resulting in isolated wrist and/or finger movements (UAT 3 to 4). This second 6-week period is followed by a (third) assessment.

\section{B 1. Training patients with a severely affected arm-hand}

The initial level of paresis is generally regarded as the most important predictor for motor recovery [35]. When neurophysiological recovery is absent, patients may be left with a non-functional arm-hand that cannot be used in daily activities. It is not useful to train patients in program 1 under the same practice conditions and as intensively as patients in programs 2 and 3.

Patients in program 1 spend about 4.5 hours a week on training. The training consists of the following topics: Education about the basic principles of how the affected arm is related to the body and mind, and why it is not moving adequately; education and exercises on how to position the arm-hand in different circumstances and postures (e.g., lying in bed, or in sport or vocational situations); exercises to avoid discomfort, maintaining joint mobility and maintaining muscles/tendons in an optimal condition; exercises to provoke voluntary movement where possible; learning strategies on what to do when discomfort nevertheless arises; and training in the use of supportive tools like static or dynamic splints, braces and/or slings. Every day during the 5 days per week, one of these topics will be discussed in a group.

A substantial proportion of patients with a severely affected arm-hand will not regain dexterity. The difficulty patients have in dealing with this poor prognosis in the post- 
stroke sub-acute phase may complicate their treatment and deter adherence. Diminished therapy adherence will hinder the learning process. Therapist may improve adherence by helping the patient to understand his or her reactions and constraints to learned skills, having the patient adopt similar strategies used by fellow patients to cope with their severely affected arm-hand, and by providing a valid prognosis based on the patient's individual characteristics. Program 1 is based on the Attitude-Social influence self-Efficacy (ASE) model [44], which assumes that attitudes, social influences and self-efficacy expectations determine intention and behaviour. These cognitive aspects are coupled with relevant topics for patients with a severely affected arm-hand.

\section{B2. Training patients with a moderately or mildly affected arm-hand}

Patients following the 'task-oriented arm-hand performance training' receive intensive exercise training spread across 5 days per week, covering approximately 7 hours per week. In contrast to program 1, program 2 and program 3 provide patients with more training because they are generally able to work more intensively due to the functional capabilities of their affected hand and their overall better condition. In general, the recommended duration of arm-hand treatment is about 1 hour per session $[4,45,46]$. With respect to general duration and frequency of training, phases of 6 to 12 weeks are advocated $[47,48]$.

Given the program's modularity, all interventions are embedded in 60 minute time blocks, during which the patient starts with the set-up of the training of a personal goal for at least 5 minutes, followed by 40-50 minutes of training exercises, related to the personal goal. Immediately after this training session, the patient works for at least 5 minutes towards the same personal goal again. An example of a personal goal may be to handle the garden hose to water the plants.

Programs 2 and 3 target patients with, respectively, a UAT score of 2-3 and a UAT score of 4-7. Patients from both these groups are eligible for task-oriented training. This involves training in functional (skill-related) tasks with a high level of repetition, assuming an interaction between the task or skill, the patient performing the task, and the context in which the task is performed [26, 49]. Scientific evidence of task-oriented 
training being associated with neuroplastic changes is increasing [50,51], although the variety of training content, combined with different durations and intensities reported, makes it hard to compare treatment effects between interventions [52].

Clinical management of motor control problems in task-oriented training uses the following five steps:

1) Perform a task analysis together with the patient and quantify functional abilities.

2) Check the strategies used to accomplish functional skills.

3) Consider which underlying sensory, motor, or cognitive factors constrain functional movement and which factors might be the most trainable.

4) Choose a suitable motor learning approach and appropriate practice conditions.

5) Train as functionally as possible to accomplish well-defined results, based on successful transfer of the learned task from a clinical environment to the home environment [53].

The application of these five steps in arm-hand rehabilitation practice is outlined below.

Step 1: task analysis. In the first week of programs 2 and 3 the therapist establishes the level of task performance by asking the patient to perform a meaningful and attainable functional task. The focus is on whether the patient can do the task and the degree of difficulty.

The therapist determines the degree to which the patient uses the affected arm-hand during the task.

Step 2: strategies used to accomplish functional skills. During the execution of the task, the therapist analyses the task performance strategies used by the patient. After examining the patient's problem-solving strategy, small adaptations to the task may be made. In these situations the therapist examines the underlying mental and physical capacities of the patient; the mental, cognitive and motor demands of the task; the strategies used by the patient to meet these demands, and their ability to choose the most efficient strategy for a given task [53].

Step 3: constellation of impairments. The multitude of underlying cognitive and/or sensorymotor deficits that contribute to the ability to use the affected arm-hand in real life activities are determined by using the Action Model of Goal-directed Movement [54]. Subsequently, 
the Hypothesis-Oriented Clinical Practice method $[55,56]$ is used to establish an adequate starting point for the interventions. Specific exercises for the patient will be set up in the first week in accordance with the patient's individual needs and the existing capabilities of the paretic arm-hand.

Step 4: practical applications of motor learning. Based on the outcome of the first three steps, a suitable learning approach and concomitant practice conditions are chosen, taking into account that a) the focus of practice lays on the execution of functional tasks [15], b) in most cases retraining motor skills demands large amounts of practice and a great deal of time spent on arm-hand therapy [57, 58], and c) the training starts in an early phase of rehabilitation, thereby avoiding learned nonuse and the development of abnormal movement patterns as well as preventing secondary symptoms $[11,59]$.

Step 5: transfer to real life performance. Transferring the goals set during the period of training to both a new task and the patient's situation is vital and strongly influences treatment success. Facilitating transfer is done by setting goals that are meaningful and feasible from the patient's perspective. In addition, there must be a need to execute these goal-related tasks regularly. This is done by setting up practice conditions that closely resemble the demands of the patient's personal situations in his or her daily environment. To further enhance these practice conditions, patients are encouraged to bring their own materials and instruments to the program. Homework assignments are listed and distributed on Fridays, before going on weekend leave, and evaluated the next Monday, thus providing new insights into the operational capabilities of the affected arm-hand in home situations.

In programs 2 and 3, the effects of task-specific training may generalize towards other, untrained tasks [60]. A way to support this generalization is to stimulate the patient's awareness of the operational capability of the affected hand in a positive way in order to increase spontaneous use of this hand in an early phase during rehabilitation [61]. To do this, patients are offered and employ a broad variety of frequently used and familiar tasks that they face in daily practice in their home environment and, in most cases, also during their rehabilitation period. For this purpose, six intervention modules have been developed. Each module contains a selection of tasks, specifically addressing personal goals (see Figure 3). 


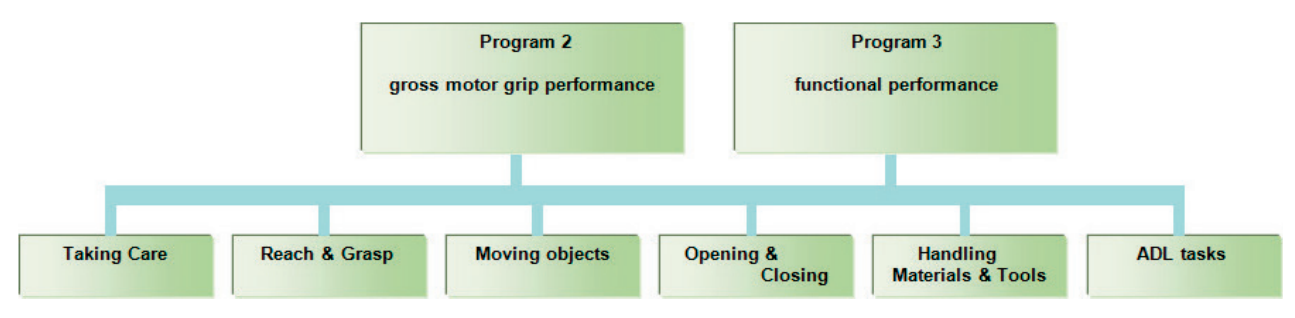

Figure 3. Six training modules used in programs 2 and 3 of CARAS

The first module ('taking care and prevention') focuses on improving and maintaining an optimal condition of the paretic arm-hand. Compared to the version in program 1, this module contains different exercises aimed at getting and keeping the affected shoulder and arm-hand supple and free of pain, which is also relevant for persons with a higher functional arm-hand. The other five modules are aimed at improving arm-hand skills performance, targeting reach and grasp tasks, moving objects, opening and closing items (e.g. door, drawer, zipper, buttons); handling materials and handoperated tools (like screwdrivers, hairdryer) and completing activities of daily living (e.g. using towel, toothbrush). The tasks are grouped into three levels of difficulty (easy, medium and hard) and are based on the stages described in the Fugl-Meyer Motor Assessment [43].

\section{Self-efficacy}

A contemporary method for improving patients' ability level is promoting their selfefficacy. Self-efficacy is described as confidence in one's ability to perform a task or exert a specific behaviour [36]. Many interventions that enhance self-efficacy may elicit positive effects on peoples' outcome after stroke $[37,62,63]$ as applied in task-oriented training methods [64] or group education interventions [65]. Therapists can incorporate principles of self-efficacy for improving patients' empowerment. By extracting and defining goals, patients are enabled to work toward their goals, boosting their belief in 
improvement. This may result in patients integrating their affected hand in daily activities. The self-efficacy principles, explained below, are essential to the success of Part 2 of CARAS and should be integrated in daily routine practice.

1. Mastery experiences: positive experiences with a task/skill

2. Vicarious experience: comparison of oneself to other patients

3. Verbal persuasion: encouragement of the patient during exercising

4. Physiological feedback: beliefs formed from feedback produced by the patient's own physiologic state [66].

\section{Mastery experience}

The development of efficacy beliefs through enactive experience creates effective performance [66]. Therefore, therapists should strive to creating patient involvement and motivation during the therapy programs [67, 68]. Prior to the training program, a semi-structured interview is performed to extract three to six activities that are both meaningful and challenging to that patient. The important characteristics of these activities are that they have to be used frequently and be directly related to homebased activities in daily life. These activities are converted into attainable and meaningful goals and are rated by the patient on a six-point ordinal (Likert) scale varying from very easy to perform to very difficult to perform.

Complex skills are often broken down into subskills that are easier to master. These subskills (part practice) are subsequently presented in a chronologic sequence to acquire or recover the complex skill (whole practice) [66]. Progression towards each goal is monitored in a personal training diary, rated by the patient three times a week using a quantitative measure such as a visual analogue scale or the time used to perform the goal. At the end of each week, progression in goal-attainment is visualised graphically. Identifying even small steps made towards the goals is done by patients themselves to stimulate confidence and to maintain a positive trend regarding their self-perceived ability level [69]. After six weeks, all goals set are re-evaluated and rated using a 6-point Likert scale. 


\section{Vicarious experience}

Observational practice can make unique and important contributions to learning, especially when the observation is combined with physical practice [70]. In CARAS' programs 2 and 3 , the patient works towards their individual goals in groups of patients who experience similar motor impairments. Each individual is able to observe the other while exercising. Working on an identical activity level facilitates learning through mutual observation and reduces insecurity through role modelling and dyad practices [71]. Another way to provide vicarious experiences is to have the patient observe himself as a model using video recordings taken during different time epochs, during one or more sessions.

To obtain persuasive model conditions it is important to create certain similarities in the training sessions (e.g., two patients who have a similar goal, like eating with knife and fork). The third method of providing vicarious experiences is to use the therapist as a role model, especially in situations that warrant giving extrinsic feedback as a way to increase knowledge of performance [53].

\section{Verbal persuasion}

Feedback that emphasizes successful performance and ignores less successful attempts benefits learning and may boost motivation [66]. During training, small progressions can be noticed and conveyed as positive feedback to the patient. Patients are also taught to identify these small progressions and to provide positive feedback to themselves and, when possible, to fellow patients. Positive family and social support may improve motivation and functional recovery [72]. CARAS encourages family to visit sessions. Furthermore, homework assignments are provided so that in homebased situations during weekends the family can notice and encourage skills mastered by the patient. 


\section{Physiological feedback}

Somatotopic maps (i.e., body schemes) are not rigid, but are subject to constant modification, depending on experience, and are updated during movement [73]. Besides the loss of voluntary movement in the affected arm-hand, in the first weeks post-stroke the loss of touch detection and proprioception has been noted in a high proportion of patients [74]. During this phase, patients often cope with a changed perception of the affected arm-hand [75], which influences their own judgement on the functional capability of the arm-hand. Enhancing patients' confidence in performing tasks can be achieved by adjusting perceptions [76].

Maintaining or improving these perceptions is done by: a) explaining (physiological) mechanisms underlying symptoms like co-contraction, lack of proprioception and/or voluntary movements, experienced by the patient during skill performance, b) providing interventions in situ fitted to the most relevant problem (e.g., providing additional muscle strength training during a functional task training like cutting meat); and $c$ ) relating progressions made to relevant tasks.

\section{The swift implementation of interventions}

In order to keep up with the state-of-the-art evidence and to test new developments, CARAS can be easily adapted without having to resort to major (systemic) alterations like the time of treatment or the (chronological) content of treatment.

CARAS consists of time blocks in which the type of training is defined. Other blocks with different content can easily replace parts of these blocks and their content. However, starting with setting personal goals and evaluating these goals again after a period of training should be maintained. Working in time blocks makes it easier to respond quickly to new developments, like the use of aids. Removing a single training component and replacing it by another component provides valuable insight into the added value of that component. Effectiveness of the newly implemented component can be evaluated by using the assessments on performance level and capacity level (presented in Section B), and the quantitative self-evaluation method (outlined in Section C). 


\section{Discussion}

The aim of this paper was to present a modular and clinically manageable arm-hand rehabilitation framework (called: CARAS) that can be used by paramedical staff to structure and implement their treatment of arm-hand function and arm-hand skill performance problems in stroke survivors. Four common problems clinicians face during the rehabilitation of stroke are tackled: the heterogeneity in dexterity of the affected armhand, the lack of the patient's engagement with therapy, the non-transparency of procedures, and the slow response to innovations [11, 20, 77, 78].To provide answers to these problems, self-efficacy principles and task-oriented training methods have been merged into in a modular program, stratified for level of arm-hand impairment, and designed to be easily adaptable in response to innovations.

CARAS induces at least three changes in the rehabilitation of stroke survivors. First, different patterns of recovery of arm-hand function imply that individual patients will have different rehabilitation needs [79]. With respect to the heterogeneity among these recovery patterns, the use of dexterity levels (based on UAT scores) is helpful for therapists to target specific (motor) problems related to the paretic arm-hand [11]. Stratification, based on the presence of dexterity and corresponding functional possibilities, facilitates a better focus and tailored therapy delivery.

Second, to create an optimal state of readiness in patients, self-efficacy principles are embedded in CARAS. The effectiveness of self-efficacy principles, however, is not clear [80]. In line with several other studies [65, 81], four sources of self-efficacy are applied in the three programs constituting CARAS. However, when adapting these sources of selfefficacy during training, it is vital to keep in mind that some patients may not able to understand all aspects of self-efficacy. As a result of their stroke, patients may experience cognitive and/or mood disorders. Mood states can bias attention and can affect how events are interpreted, cognitively organized and retrieved from memory [82, 83]. Cognitive disorders are a major exclusion criterion for most studies in which self-efficacy or self-management interventions are examined [80]. In CARAS, patients with cognitive disorders are included.

Mastery experiences is the most important source of efficacy information because successes build a robust belief in one's personal efficacy[36]. Therefore, this source is 
used constantly, independent of problems perceived and exercises performed. The other three sources - vicarious experience, verbal persuasion and physiological feedback - are used more intermittently, depending on the patient's cognitive status and mood status.

Third, the task-oriented training method combined with the self-efficacy principles used in Part 2 of CARAS lead to a condensed organisational structure that resolves several logistical issues. Patients commit themselves to be present during a minimum of three sessions weekly for six consecutive weeks and, in line with task-oriented training, a minimum of 40-50 minutes of training should be provided during each session. CARAS' modular structure, coupled with its clear timing and concomitant assessments, provides the patient, his or her family or partner, and the therapist with valuable insight into any progress made, the prognosis of the impaired arm-hand and (additional) therapy requirements.

Finally, following the implementation criteria for using technology in rehabilitation [84] facilitates the use of technology in CARAS. Its modular structure allows for quick implementation of new interventions. Stratification of patients into three groups (severely, moderately and mildly impaired) makes it easier to match quickly new technologies to patients' needs [85]. The added value of new technologies may be evaluated in the clinical setting by using standard, objective measures in the assessment phases.

There is a variety of existing arm-hand programs, each tackling one or more of the aforementioned problems. For example, the ICARE protocol [86] and the task-specific training method of [16] both provide a structured framework and customized therapy with challenging activities related to the real-world tasks chosen by the patient. The amount of training time in the BATRAC bilateral arm training [87] shows similarities with the training intensity of CARAS' programs 2 and 3 . The upper extremity treatment program of Wallace [88] contains training blocks and a stratification of patients with a mildly affected arm-hand into three levels, thereby accommodating graded functional training. McDonnell et al., (2013) incorporated a hypothetico-deductive framework in their arm-hand program [20].

To our knowledge, however, there is no program that targets all four problems and their concomitant solutions as presented above. CARAS merges experience-based clinical treatment with evidence-based interventions, targeting clearly defined populations and 
covering a substantial part of the stroke population with an affected arm-hand. Its theoretical framework makes it easier to identify clear targets or goals toward which the patients can train. CARAS provides a systematic approach to therapy and accommodates appropriate evaluation methods for evaluating novel developments to be implemented.

\section{Considerations}

Some considerations regarding this framework should be mentioned. Although the initial results relating to progressions made during and after therapy are hopeful, the added value of CARAS, compared to other existing arm-hand programs, has not yet been evaluated. It has, however, been successfully implemented at Adelante Rehabilitation Centre.

The second limitation is that the program requires a certain number of therapy hours for six consecutive weeks. This six-week schedule demands regular timing in a patient's therapy schedule, which may sometimes conflict with other therapy related to stroke rehabilitation in the sub-acute phase.

\section{Future Research}

Future research will focus on a) evaluating the outcomes of CARAS, comparing it to other arm-hand treatment programs in stroke, b) implementation in additional centres, and c) further optimisation of CARAS regarding the dose-response relationship and the optimization of the number of hours involved in 'face-to-face' therapy delivery.

\section{Conflict of interest}

The authors declare that they have no competing interests. 


\section{References}

1. Broeks JG, Lankhorst GJ, Rumping K, Prevo AJ. The long-term outcome of arm function after stroke: results of a follow-up study. Disability and rehabilitation. 1999;21(8):357-64. PubMed PMID: 10503976.

2. Johansson A, Mishina E, Ivanov A, Bjorklund A. Activities of daily living among St Petersburg women after mild stroke. Occup Ther Int. 2007;14(3):170-82. PubMed PMID: 17621375.

3. Lai SM, Studenski S, Duncan PW, Perera S. Persisting consequences of stroke measured by the Stroke Impact Scale. Stroke; a journal of cerebral circulation. 2002;33(7):1840-4. PubMed PMID: 12105363.

4. Pang MY, Harris JE, Eng JJ. A community-based upper-extremity group exercise program improves motor function and performance of functional activities in chronic stroke: a randomized controlled trial. Archives of physical medicine and rehabilitation. 2006;87(1):19. PubMed PMID: 16401430.

5. Wolfe CD. The impact of stroke. Br Med Bull. 2000;56(2):275-86. PubMed PMID: 11092079.

6. Nichols-Larsen DS, Clark PC, Zeringue A, Greenspan A, Blanton S. Factors influencing stroke survivors' quality of life during subacute recovery. Stroke; a journal of cerebral circulation. 2005;36(7):1480-4. PubMed PMID: 15947263.

7. WHO. International classification of functioning, disability and health. 2001.

8. Albert SJ, Kesselring J. Neurorehabilitation of stroke. J Neurol. 2012;259(5):817-32. PubMed PMID: 21964750.

9. Brewer L, Horgan F, Hickey A, Williams D. Stroke rehabilitation: recent advances and future therapies. An International Journal of Medicine. 2013;106(1):11-25. doi: 10.1093/qjmed/hcs174.

10. Hömberg V. neurorehabilitation approaches to facilitate motor recovery. 3rd ed. Amsterdam: Elsevier 2013.

11. Langhorne P, Bernhardt J, Kwakkel G. Stroke rehabilitation. Lancet. 2011;377:1693 - 702.

12. Dobkin BH. Strategies for stroke rehabilitation. Lancet Neurol. 2004;3(9):528-36. PubMed PMID: 15324721.

13. Shephard R, Carr J. Bridging the gap between theory and practice. In: Refshauge Kathryn, editor. Science-Based Rehabilitation-Theories into Practice. Edinburgh: ButterworthHeinemann; 2005. p. 1-13. 
14. Sterr A, Szameitat A, Shen S, Freivogel S. Application of the CIT concept in the clinical environment: hurdles, practicalities, and clinical benefits. Cogn Behav Neurol. 2006;19(1):48-54. PubMed PMID: 16633019.

15. Van Peppen RP, Kwakkel G, Wood-Dauphinee S, Hendriks HJ, Van der Wees PJ, Dekker J. The impact of physical therapy on functional outcomes after stroke: what's the evidence? Clinical rehabilitation. 2004;18(8):833-62. PubMed PMID: 15609840.

16. Arya KN, Verma R, Garg RK, Sharma VP, Agarwal M, Aggarwal GG. Meaningful taskspecific training (MTST) for stroke rehabilitation: a randomized controlled trial. Topics in stroke rehabilitation. 2012;19(3):193-211. PubMed PMID: 22668675.

17. Combs SA, Kelly SP, Barton R, Ivaska M, Nowak K. Effects of an intensive, task-specific rehabilitation program for individuals with chronic stroke: a case series. Disability and rehabilitation. 2010;32(8):669-78. PubMed PMID: 20205581.

18. Davis JZ. Task selection and enriched environments: a functional upper extremity training program for stroke survivors. Topics in stroke rehabilitation. 2006;13(3):1-11. PubMed PMID: 16987787.

19. Harris JE, Eng JJ, Miller WC, Dawson AS. A self-administered Graded Repetitive Arm Supplementary Program (GRASP) improves arm function during inpatient stroke rehabilitation: a multi-site randomized controlled trial. Stroke; a journal of cerebral circulation. 2009;40(6):2123-8. PubMed PMID: 19359633.

20. McDonnell M, Hillier S, Esterman A. Standardizing the Approach to Evidence-Based Upper Limb Rehabilitation after Stroke. Topics in stroke rehabilitation. 2013;20(5):432-40.

21. Platz T. Impairment-oriented Training (IOT) - scientific concept and evidence-based treatment strategies. Restorative Neurology and Neuroscience. 2004;22:301-15.

22. French B, Thomas LH, Leathley MJ, Sutton CJ, McAdam J, Forster A, et al. Repetitive task training for improving functional ability after stroke. The Cochrane database of systematic reviews. 2007;(4):CD006073. PubMed PMID: 17943883.

23. Timmermans $A$, Seelen $H$, Kingma $H$. Task-oriented training: An essential element in technology-supported rehabilitation of skilled arm-hand performance after stroke. IEEEEMBS Benelux Chapter Symposium; 2009 November 9-10, 2009; Twente.

24. Winstein CJ, Rose DK, Tan SM, Lewthwaite R, Chui HC, Azen SP. A randomized controlled comparison of upper-extremity rehabilitation strategies in acute stroke: A pilot study of immediate and long-term outcomes. Archives of physical medicine and rehabilitation. 2004;85(4):620-8. PubMed PMID: 15083439.

25. Wolf SL, Winstein CJ, Miller JP, Thompson PA, Taub E, Uswatte G, et al. Retention of upper limb function in stroke survivors who have received constraint-induced movement therapy: the EXCITE randomised trial. Lancet Neurol. 2008;7(1):33-40. PubMed PMID: 18077218. 
26. Timmermans AA, Seelen HA, Willmann RD, Bakx W, de Ruyter B, Lanfermann G, et al. Arm and hand skills: Training preferences after stroke. Disability and rehabilitation. 2009;31(16):1344-52. PubMed PMID: 19479535.

27. Timmermans AA, Seelen HA, Willmann RD, Kingma H. Technology-assisted training of arm-hand skills in stroke: concepts on reacquisition of motor control and therapist guidelines for rehabilitation technology design. Journal of neuroengineering and rehabilitation. 2009;6:1. PubMed PMID: 19154570.

28. Winstein CJ, Stewart JC. Textbook of Neural Repair and Rehabilitation Cambridge: Cambridge University Press; 2006. 89-102 p.

29. Magill R. Transfer of Learning. Motor Learning and Control Concepts and Applications. Boston: Mc Graw Hill; 2007. p. 290-306.

30. Berwick D. Dissiminating Innovations in Health Care. Journal of American Medical Association. 2003;289(15):1969 - 75.

31. Cheeran B, Cohen L, Dobkin B, Ford G, Greenwood R, Howard D, et al. The future of restorative neurosciences in stroke: driving the translational research pipeline from basic science to rehabilitation of people after stroke. Neurorehabilitation and neural repair. 2009;23(2):97-107. PubMed PMID: 19189939.

32. Nutley S, Walter I, Davies H. From Knowing to Doing: A Framework for Understanding the Evidence-into-Practice Agenda. Evaluation. 2003;9:125-48.

33. Herzlinger RE. Why innovation in health care is so hard. Harv Bud Rev. 2006;84(5):58-66.

34. Hayward K, Barker R, Brauer S. Interventions to promote upper limb recovery in stroke survivors with severe paresis: a systematic review. Disability and rehabilitation. 2010;32(24):1973-86. PubMed PMID: 20964563.

35. Nijland RH, van Wegen EE, Harmeling-van der Wel BC, Kwakkel G. Presence of finger extension and shoulder abduction within 72 hours after stroke predicts functional recovery: early prediction of functional outcome after stroke: the EPOS cohort study. Stroke; a journal of cerebral circulation. 2010;41(4):745-50. Epub 2010/02/20. doi: 10.1161/ strokeaha. 109.572065. PubMed PMID: 20167916.

36. Bandura A. Self-efficacy. Ramachaudran V, editor. New York: Academic Press (Reprinted in H. Friedman [Ed.]; 1994.

37. Jones F, Rianzi A. Self-efficacy and self-management after stroke: a systematic review. Disability and Rehabililation. 2011;33(10).

38. Kristensen H, Persson D, Nygren C, Boll M, Matzen P. Evaluation of evidence within occupational therapy in stroke rehabilitation. Scandinavian Journal of Occupational Therapy. 2011;18:11-25. 
39. Kruitwagen-van Reenen ET, Post MW, Mulder-Bouwens K, Visser-Meily JM. A simple bedside test for upper extremity impairment after stroke: validation of the Utrecht Arm/Hand Test. Disability and rehabilitation. 2009;31(16):1338-43.

40. Warlow C, Sudlow C, Dennis M, Wardlaw J, Sandercock P. Stroke. Lancet. 2003; 362(9391):1211-24. PubMed PMID: 14568745.

41. Penta M, Tesio L, Arnould C, Zancan A, Thonnard JL. The ABILHAND questionnaire as a measure of manual ability in chronic stroke patients: Rasch-based validation and relationship to upper limb impairment. Stroke; a journal of cerebral circulation. 2001;32(7):1627-34. PubMed PMID: 11441211.

42. Lyle RC. A performance test for assessment of upper limb function in physical rehabilitation treatment and research. Int J Rehabil Res. 1981;4(4):483-92. PubMed PMID: 7333761.

43. Fugl-Meyer AR, Jaasko L, Leyman I, Olsson S, Steglind S. The post-stroke hemiplegic patient. 1. a method for evaluation of physical performance. Scand J Rehabil Med. 1975;7(1):13-31. PubMed PMID: 1135616.

44. de Vries H, Dijkstra M, Kuhlman P. Self-efficacy: the third factor besides attitude and subjective norm as a predictor of behavioural intentions. Health education Research. 1988;3:273 - 82 .

45. Kwakkel G, Kollen B, Lindeman E. Understanding the pattern of functional recovery after stroke: facts and theories. Restor Neurol Neurosci. 2004;22(3-5):281-99. PubMed PMID: 15502272.

46. Duncan P, Studenski S, Richards L, Gollub S, Lai SM, Reker D, et al. Randomized clinical trial of therapeutic exercise in subacute stroke. Stroke; a journal of cerebral circulation. 2003;34(9):2173-80. PubMed PMID: 12920254.

47. Beachle TR, Earle RW, Wathen D. Essentials of strenght training and conditioning. 3th ed. Champaign: Human Kinetics; 2008.

48. Kisner C, Colby L. Strategies for effective exercise and task-specific instruction. Therapeutic exercise Foundations and techniques. Philadelphia: F.A. Davis Company; 2007. p. 29.

49. Shumway-Cook A, Woollacott M. Motor learning and recovery of function. In: Motor control Translating research into clinical practice. Philadelphia: Lippincott Williams \& Wilkins; 2007. p. 21-45.

50. Jang SH, Kim YH, Cho SH, Lee JH, Park JW, Kwon YH. Cortical reorganization induced by task-oriented training in chronic hemiplegic stroke patients. Neuroreport. 2003; 14(1):137-41. PubMed PMID: 12544845. 
51. Richards LG, Stewart KC, Woodbury ML, Senesac C, Cauraugh JH. Movementdependent stroke recovery: a systematic review and meta-analysis of TMS and fMRI evidence. Neuropsychologia. 2008;46(1):3-11. PubMed PMID: 17904594.

52. French B, Leathley M, Sutton C, McAdam J, L Thomas, Forster A, et al. A systematic review of repetitive functional task practice with modelling of resource use, costs and effectiveness. Health Technology Assessment 2008;12(30).

53. Shumway-Cook A, Woollacott M. Motor Control Translating Research into Clinical Practice. Third ed: Wolters Kluwer/ Lippincott Williams \& Wilkins; 2007. P. 576.

54. Smits-Engelsman BCM, Galen GP, Hulstijn RGJ. Motorisch onderzoek door de fysiotherapeut: Het belang van procesgeorienteerd, kwantitatief en kwalitatief motorisch onderzoek. Aufdemkampe GdD, J.B. van Ham, I. Smits-Engelsman, B.C.M. Vaes, P. editor. Diegem: Bohn Stafleu Van Loghum; 1997.

55. Elstein A, Shulman L, Sprafka S. Medical problem solving: An analysis of clinical reasoning. Cambridge, MA: Harvard University Press; 1978.

56. Arocha J, Patel V, Patel Y. Hypothesis generation and the coordination of theory and evidence in novice diagnostic reasoning. Medical Decision Making. 1993;13(3):198-211. doi: $10.1177 / 0272989 \times 9301300305$.

57. Kwakkel G, Wagenaar RC, Koelman TW, Lankhorst GJ, Koetsier JC. Effects of intensity of rehabilitation after stroke. A research synthesis. Stroke; a journal of cerebral circulation. 1997;28(8):1550-6. PubMed PMID: 9259747.

58. Kwakkel G, Wagenaar RC, Twisk JW, Lankhorst GJ, Koetsier JC. Intensity of leg and arm training after primary middle-cerebral-artery stroke: a randomised trial. Lancet. 1999;354(9174):191-6. PubMed PMID: 10421300.

59. Rodgers H, Mackintosh J, Price C, Wood R, McNamee P, Fearon T, et al. Does an early increased-intensity interdisciplinary upper limb therapy programme following acute stroke improve outcome? Clinical rehabilitation. 2003;17(6):579-89. PubMed PMID: 12971702.

60. Schaefer SP, C. Lang, C. Transfer of Training Between Distinct Motor Tasks After Stroke: Implications for Task- Specific Approaches to Upper-Extremity Neurorehabilitation. Neurorehabil Neural. 2013;27(7):602-12.

61. Taub E, Uswatte G. Constraint-Induced Movement therapy: answers and questions after two decades of research. NeuroRehabilitation. 2006;21(2):93-5. PubMed PMID: 16917156

62. Korpershoek C, van der Bij J, Hafsteinsdóttir T. Self-efficacy and its influence on recovery of patients with stroke: a systematic review. Journal of Advanced Nursing. 2011;67(9):1876-94. doi: 10.1111/j.1365-2648.

63. Jones F. Strategies to enhance chronic disease self-management: how can we apply this to stroke? Disability and rehabilitation. 2006;28(13-14):841-7. PubMed PMID: 16777771. 
64. Salbach NM, Mayo NE, Robichaud-Ekstrand S, Hanley JA, Richards CL, WoodDauphinee $S$. The effect of a task-oriented walking intervention on improving balance selfefficacy poststroke: a randomized, controlled trial. J Am Geriatr Soc. 2005;53(4):576-82. PubMed PMID: 15817001.

65. Kendall E, Catalanoa T, Kuipers P, Posnerb N, Buysa N, Charker J. Recovery following stroke: The role of self-management education. Social Science \& Medicine 2007;64:735 - 46.

66. Bandura A. Self-efficacy: the exercise of control. New York: W.H Freeman and Company; 1997.

67. Locke EA, Latham GP. Building a practically useful theory of goal setting and task motivation. A 35-year odyssey. Am Psychol. 2002;57(9):705-17. PubMed PMID: 12237980.

68. Sivaraman Nair KP. Life goals: the concept and its relevance to rehabilitation. Clinical rehabilitation. 2003;17(2):192-202. PubMed PMID: 12625660.

69. van de Laar KE, van der Bijl JJ. Strategies enhancing self-efficacy in diabetes education: a review. Sch Inq Nurs Pract. 2001;15(3):235-48. PubMed PMID: 11871582.

70. Shebilske WL, Regian JW, Arthur WJ, Jordan JA. A Dyadic Protocol for Training Complex Skills. The Journal of the Human Factors and Ergonomics Society. 1992;34:369-74.

71. Shea $\mathrm{CH}$, Wright DL, Wulf G, Whitacre C. Physical and observational practice afford unique learning opportunities. J Mot Behav. 2000;32(1):27-36. PubMed PMID: 11008269.

72. Tsouna-Hadjis E, Vemmos KN, Zakopoulos N, Stamatelopoulos S. First-stroke recovery process: the role of family social support. Archives of physical medicine and rehabilitation. 2000;81(7):881-7. PubMed PMID: 10895999.

73. Haggard P, Wolpert, DM. Disorders of Body Scheme. In: Freund HJJ, M. Hallett, M. Leiguarda, R, editors. Higher- order motor disorders from neuroanatomy and neurobiology to clinical neurology. 1. oxford: Oxford University Press; 2005.

74. Carey LM. Somatosensory Loss after Stroke. Physical and Rehabilitation Medicine. 1995;7(1):51-91.

75. Longo MR, Azanon E, Haggard P. More than skin deep: body representation beyond primary somatosensory cortex. Neuropsychologia. 2010;48(3):655-68. PubMed PMID: 19720070.

76. Souza $D$. The effects of sensation and motivation on regaining movement control following stroke. physiotherapy. 1983;69(238).

77. Kuipers K, McKenna K. Upper Limb Rehabilitation following Brain Injury: Complex, Multifaceted and Challenging. British Journal of Occupational Therapy. 2008;72(1):20-8.

78. Oujamaa L, Relave I, Froger J, Mottet D, Pelissier JY. Rehabilitation of arm function after stroke. Literature review. Annals of physical and rehabilitation medicine. 2009;52(3):26993. PubMed PMID: 19398398. 
79. Meldrum D, Pittock SJ, Hardiman O, Ni Dhuill C, O'Regan M. Recovery of the upper limb post ischaemic stroke and the predictive value of the Orpington Prognostic Score. Clinical rehabilitation. 2004;18(6):694-702. PubMed PMID: 15473121.

80. Boger EJ, Demain S, Latter S. Self-management: a systematic review of outcome measures adopted in self-management interventions for stroke. Disability \& Rehabilitation. 2013;35(16-17):1415-28.

81. Dixon G, Thornton EW, Young CA. Perceptions of self-efficacy and rehabilitation among neurologically disabled adults. Clinical rehabilitation. 2007;21:230-40.

82. Bower GH. Affect and cognition. B PTotRSoL, editor. London 1983. 387-402 p.

83. Eich E. Searching for Mood Dependent Memory. Psychological Science. 1995;6(2):67-75.

84. Hochstenbach-Waelen A, Seelen HAM. Embracing change: practical and theoretical considerations for successful implementation of technology assisting upper limb training in stroke. Journal of neuroengineering and rehabilitation. 2012;9(52):1-12.

85. Brewer BR, McDowell SK, Worthen-Chaudhari CL. Poststroke upper extremity rehabilitation: a review of robotic systems and clinical results. Top Stroke Rehabilitation. 2007; 14:22-44.

86. Winstein CJ, Wolf SL, Dromerick AW, Lane CJ, Nelsen MA, Lewthwaite R, et al. Interdisciplinary Comprehensive Arm Rehabilitation Evaluation (ICARE): a randomized controlled trial protocol. BMC Neurol. 2013;13:5. PubMed PMID: 23311856.

87. van Delden AL, Peper CL, Nienhuys KN, Zijp NI, Beek PJ, Kwakkel G. Unilateral versus bilateral upper limb exercise therapy after stroke: A systematic review. J Rehabil Med. 2011;44:106-17.

88. Wallace AC, Talelli P, Dileone M, Oliver R, Ward N, Cloud G, et al. Standardising the intensity of upper limb treatment in rehabilitation medicine. Clinical rehabilitation. 2010;24(5):471-8. 



\section{Chapter}

\section{Changes in arm-hand function and arm-hand skill performance in patients after stroke during and after rehabilitation}

Johan Anton Franck, Rob Johannes Elise Marie Smeets, Henk Alexander Maria Seelen 


\section{Abstract}

Background: Arm-hand rehabilitation programs applied in stroke rehabilitation frequently target specific populations and thus are less applicable in heterogeneous patient populations. Besides, changes in arm-hand function (AHF) and arm-hand skill performance (AHSP) during and after a specific and well-described rehabilitation treatment are often not well evaluated.

Method: This single-armed prospective cohort study featured three subgroups of stroke patients with either a severely, moderately or mildly impaired AHF. Rehabilitation treatment consisted of a Concise Arm and hand Rehabilitation Approach in Stroke (CARAS). Measurements at function and activity level were performed at admission, clinical discharge, 3, 6, 9 and 12 months after clinical discharge.

Results: Eighty-nine stroke patients (M/F:63/23; mean age:57.6yr (+/-10.6); poststroke time:29.8 days (+/-20.1)) participated. All patients improved on AHF and armhand capacity during and after rehabilitation, except on grip strength in the severely affected subgroup. Largest gains occurred in patients with a moderately affected AHF. As to self-perceived AHSP, on average, all subgroups improved over time. A small percentage of patients declined regarding self-perceived AHSP post-rehabilitation.

Conclusion: A majority of stroke patients across the whole arm-hand impairment severity spectrum significantly improved on AHF, arm-hand capacity and selfperceived AHSP. These were maintained up to one year post-rehabilitation. Results may serve as a control condition in future studies. 


\section{Introduction}

One of the most common deficits following stroke is a persistent impairment of the arm and hand due to a hemiparesis, which has a significant impact on performance in daily life activities [1]. Recovery of arm-hand function and skills is a major rehabilitation and health care challenge. Motor rehabilitation approaches for arm-hand performance after stroke has been changing substantially over the last decades. However, an integral arm-hand skill training approach, accommodating both the heterogeneity of the patient population and its associated patterns and levels of recovery directly post-stroke seems to be absent. A large number of well-explored and well-investigated examples of training approaches in specific (sub) populations have been identified [2] like, for instance, task-oriented training [3], mental practice [4] and constraint-induced movement therapy (CIMT) [5]. In task-oriented approaches specific functional, skillrelated tasks are trained. This is done preferably by using real-life objects [6], thereby teaching patients to solve specific problems related to, e.g., anticipatory motor adjustments or cognitive processing by using efficient goal-oriented movement strategies $[7,8]$.

Existing task-oriented arm-hand programs (e.g. [9-16]) are valuable contributions to rehabilitation practice and may offer a stable point of departure for clinicians to select the most appropriate therapy for a particular patient.

However, several aspects make it difficult for clinicians to choose the most appropriate arm-hand therapy intervention(s) for a particular patient: 1) Most studies or programs target specific populations (in particular those with some preservation of wrist and/or finger extension) and thus are less applicable for patients with a more severely affected arm-hand as seen in the heterogeneous populations of many rehabilitation centres [17]. 2) Programs are focused on either the arm or the hand alone. 3) Most of the current studies in research projects feature strictly protocolled interventions, which cannot be easily adopted in the clinicians' daily practice. 4) The lack of information about the proportional improvement or deterioration to be expected in stroke survivors in the sub-acute phase after stroke may lead to difficulties for clinicians to make decisions about arm-hand treatment objectives and concomitant prognostics regarding arm-hand skill performance. 
In order to overcome these four drawbacks a Concise Arm and hand Rehabilitation Approach in Stroke (acronym: CARAS) [18] was developed in order to guide clinicians, during their daily practice, in systematically designing a patient's optimal arm-hand rehabilitation program. CARAS is based on four constructs: a) stratification of the patient population is based on the severity of arm-hand impairment for which the Utrecht Arm-hand Test (UAT) is used [19], b) clear focus on the individual's rehabilitation goals and concomitant potential rehabilitation treatment outcomes, c) principles of self-efficacy, and d) possibility to systematically incorporate (new) technology and new evidence-based training elements swiftly. CARAS has proven to be feasible in a number of stroke units of rehabilitation centres throughout the Netherlands.

In the present study, the term 'arm -hand function' (AHF) refers to the ICF 'body function and structures level'. The term 'arm-hand skilled performance' (AHSP) refers to the ICF activity level, covering both capacity and performance [20].

The present paper focusses on two aspects.

Firstly, during rehabilitation AHF and AHSP may improve to a certain level. However, once a stroke patient has left the rehabilitation program, his arm-hand capacity and performance may deteriorate [21]. Whereas stroke patients with mild to moderate initial impairments show an almost fixed amount of recovery after stroke, ranging up to $70 \%$ $[22,23]$, stroke patients with a more severely affected arm-hand, i.e. absence of finger extension combined with large motor impairments, strongly lag behind this recovery percentage.

Four years after stroke, $67 \%$ of stroke survivors still experience non-use or disuse of the moderately or severely affected arm-hand [24].

However, it is neither well understood at what rate such deterioration (or improvement) occurs, nor in which patient categories, i.e. patients with a certain level of arm-hand severity, this is most prominent. Answers to these questions are essential for the development of more adequate, personalised and cost-effective interventions that may augment and/or maintain arm-hand skill performance (AHSP) levels in stroke patients living in their home environment. 
Secondly, the risk of losing the opportunity to clearly define 'therapy-as-usual' (TAU) is becoming a problem in AHSP research in stroke patients. In the myriad of studies evaluating newly developed training protocols aimed at improving AHF and/or AHSP, each of these new training approaches is contrasted to some kind of TAU, the latter of which may vary widely between clinics and institutes. Even worse, often TAU is not clearly defined at all.

As the implementation of many of the tested experimental treatments progresses, the concept of 'therapy-as-usual' inevitably will be lost.

The aim of the present study was to evaluate the course AHF and AHSP take in a broad range of sub-acute stroke patients during and after rehabilitation involving a therapy-as-usual (i.e. CARAS) [18].

Three subgroups, i.e. a subgroup of patients with a severely affected arm-hand, a subgroup of patients with a moderately affected arm-hand and a subgroup of patients with a mildly affected arm-hand, were formed.

The research questions were:

1) To what extent do arm-hand function and arm-hand skill performance in stroke patients change during and after their rehabilitation involving therapy-as-usual?

2) To what extent does the rate of improvement or deterioration (over time) of armhand function and arm-hand skill performance differ between three subgroups of stroke patients, i.e. patients with either a severely, moderately or mildly affected functional arm-hand, during and after their rehabilitation involving CARAS?

\section{Methods}

\section{Design}

This study is a single-armed prospective cohort study conducted between February 2011 and May 2015. Stroke patients who experienced AHF loss and (concomitantly) AHSP loss were assessed during and up till 12 months after their protocolled rehabilitation treatment. 
This investigation has been conducted according to the principles expressed in the Declaration of Helsinki. This project was approved by the Medical Ethics Committee of Maastricht University Medical Centre in the Netherlands (dossier number NL35681.068.11).

Written informed consent was obtained from all participants prior to the start of their participation in this study.

\section{Population}

The study population consisted of a broad range of sub-acute stroke patients admitted to an inpatient stroke ward of the Adelante Rehabilitation Centre. Identification of potential participants was done by the rehabilitation specialists of the stroke unit, based on the inclusion and exclusion criteria as mentioned below. Inclusion criteria were kept to a minimum: age $\geq 18$ years; clinically diagnosed with central paresis of the arm/hand at entry in the study; ability to control sitting posture; a fair cognitive level, i.e. being able to understand the questionnaires and measurement instructions. Exclusion criteria were: additional complaints that may interfere with the execution of the measurements; no informed consent.

In this study the primary outcome measure to assess arm-hand use on the hemiparetic side in stroke patients was the ARAT [25].Given a mean difference of at least $10 \%$ between baseline values and post-intervention values, an expected standard deviation of the difference of $15 \%$, a two-sided test in a repeated-measures design, an alpha of 0.05 , a power of 0.90 , and a loss to follow-up of $10 \%$, at least 29 participants per group needed to be included in the study. For three groups 87 participants were needed.

\section{Procedures}

\section{Therapy-as-usual}

All participants followed the Concise Arm and hand Rehabilitation Approach in Stroke (CARAS) [18] as provided by the medical and paramedical staff of Adelante rehabilitation centre. CARAS spans the full range of arm-hand impairments considered for this patient group. This approach consists of a well-described program offering stepwise, transparent and comprehensible procedures, tailored to specific needs of the individual patient. 
Based on the UAT score $(0-7)$ [19], patients were allocated to one of the three subgroups (i.e. subgroup 1 = severely affected, subgroup 2 = moderately affected and subgroup 3 = mildly affected) and were allocated to one of three training programs within CARAS. Subgroup 1 followed program 1 which targets stroke survivors with an UAT score of 0 to 1, and is titled 'taking care and prevention'. It is designed for stroke survivors who are not able to use their affected arm and hand for skill performance in daily life situations (non-functional arm-hand). This program contains different topics aimed at getting and keeping the affected shoulder and arm-hand in an optimal condition and learning strategies on what to do when discomfort arises. Subgroup 2 (UAT score 2-3) was admitted to program 2 and subgroup 3 (UAT score 4-7) followed program 3. Both programs are high intensity, task-oriented arm-hand performance training programs in which patients learn to integrate their affected arm and hand in daily occupations, thus optimizing their overall functional abilities in daily situations. Patients in subgroup 2 will work on becoming more able to use their affected arm and hand for passive and active stabilisation tasks, like fixating bread while making a sandwich. Patients from subgroup 3 are already able to use their affected arm and hand instantaneously in daily situations. They will work more towards complex (bi)manual activities. Patients in program 1 spend 4.5 hours on training spread across each week. Patients following program 2 or 3 receive an intensive exercise training of 6 hours spread across each week. A more detailed description of the therapy content and basic assumptions of CARAS have been described by Franck and co-workers [18].

After baseline measurements, patients enrolled in one of the three programs and started training for six consecutive weeks. After six weeks the patient left the program and entered the second assessment. Progression made, was expressed in terms of functional goals reached, based on performance and capacity levels exceeding certain minimal clinically important thresholds as captured by outcome measurements. Depending on these results it was possible for the patient to choose for a second (and final) six weeks period of training, which was then also evaluated. 


\section{Outcome measures}

The following measurements, covering both the 'function' and 'activities' domain of the International Classification of Function, Disability and Health (ICF) (World Health Organization, 2001), were performed.

At function level, the Fugl-Meyer Motor Assessment (FMA) and dynamometry (gauging grip strength) were used. The upper extremity section of the FMA is a reliable and valid test for the assessment of arm-hand function in stroke patients at function level [26, 27]. Its score ranges from 0 to 66 . The minimal clinical important difference (MCID) for the FMA upper extremity section is 9 points (for both the affected dominant and affected non-dominant arm-hand) [28].

Grip strength of the hand was measured using the JAMAR hand dynamometer [29]. Grip strength (in N) was measured three times and the mean score was used. The MCID for grip strength were $50 \mathrm{~N}$ (affected dominant hand) and $62 \mathrm{~N}$ (affected nondominant hand) [30].

At activity level, encompassing both capacity and perceived performance, the Action Research Arm test (ARAT) and ABILHAND were used. The ARAT has been proven to be reliable, valid and sensitive to change in measuring upper limb capacity at activity level in patients with stroke [31-34]. It consists of four subtests comprising 16 grasp movements and three reaching movements to be performed by the patient. Items are scored on a 4-point scale, its sum score ranging from 0 to 57 . The MCID of the ARAT were 12 points (affected dominant arm-hand) and 17 points (affected non-dominant arm-hand) [30].

The ABILHAND is a Rasch-evaluated test to assess the manual ability in terms of the difficulty perceived by patients with hand impairments in their daily life [35]. It focuses on 23 representative unimanual or bimanual activities [36, 37]. The test is administered as a semi-structured interview, using a 3-level ordinal rating scale: impossible (0), difficult (1), and easy (2) to perform. The ABILHAND is valid, responsive and clinically useful $[35,36]$. The MCID of the ABILHAND is within a range of 0.26 to 0.35 [38]. 
Finally, one single question was used every two weeks to gauge the occurrence of any major event (for example 'flu', 'falls', etc.) that might have affected the use of the arms or hands of the patient over the past two weeks. ("Has there been any major problem during the last 2 weeks preventing you from using one or both hands?(yes/no). If so, please indicate (from a short list) which event(s)."). This question was merely used as an indicator in case of any unexplainable data shift in a patient's time series occurring. This indicator was not used in the statistical analyses.

In clinical practice and in randomized clinical trials an improvement of $10 \%$ or more on the primary outcome measure is often considered being clinically relevant (e.g. Kwakkel et al [39] [40]). As to deterioration on a primary outcome measure, no clear guidelines are available. In our study we therefore decided to use a conservative threshold of $5 \%$ in identifying any deterioration, thus making sure that even a small reduction in the outcome would be considered being a deterioration.

\section{Measurement dates}

As soon as possible after admission to the rehabilitation centre the aforementioned measurements were performed (baseline measurement $\left(T_{\mathrm{BL}}\right)$ ). Furthermore, at five additional points in time, interspaced by three months, measurements were performed, starting one week prior to discharge from the clinical and outpatient rehabilitation program ( $T C D)$, followed by $T_{3 m}\left(=T_{C D}+3\right.$ months) through $T_{12 m}\left(=T_{C D}+12\right.$ months $)$. In Figure 1 an overview of the measurement dates is given.

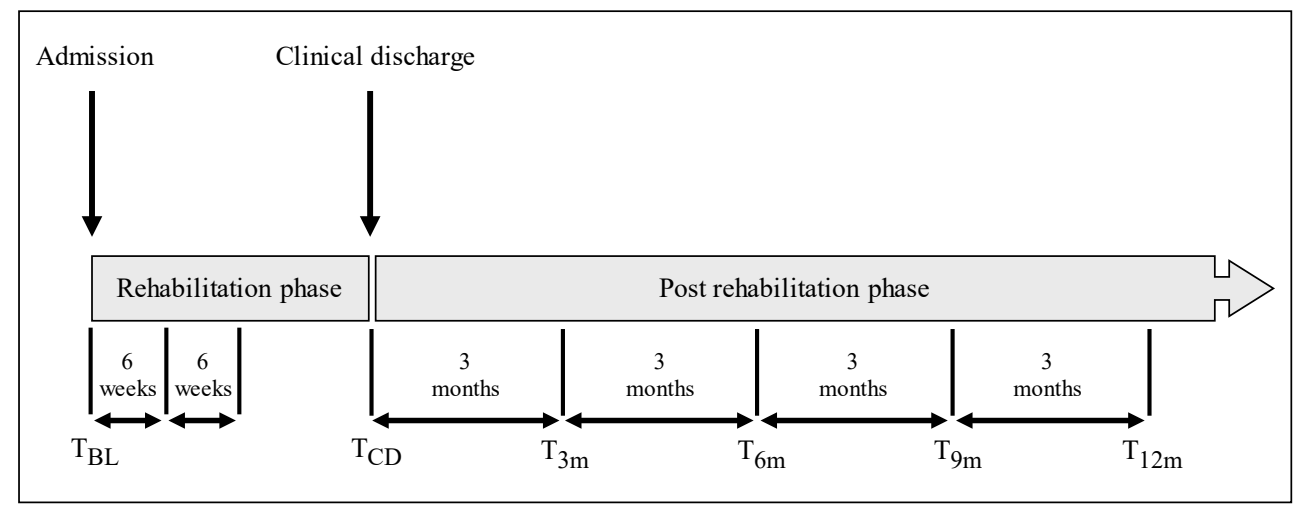

Figure 1. Overview of measurement timing. $T=$ time; $B L=$ baseline; $C D=$ clinical discharge; $m=$ month . 


\section{Data processing and statistical analysis}

For data representation, boxplots were used. As to the methodological handling of missing values in this study, four decision rules, formulated prior to the start of the study, were applied (consecutively).

1. When the baseline value was missing, its value was estimated using the mean baseline value of the (sub-)group.

2. When the $T_{12 m}$ value was missing, the 'last observation carried forward' procedure [41] was used.

3. When 1 or 2 (temporally adjacent) value(s), not being the baseline value or the $T_{12 m}$ value, were missing, these missing value(s) were estimated by linear interpolation using the two valid adjacent values in the time series.

4. In case of 3 or more missing values, the whole case was discarded.

The MCID threshold values, as used in this study, were corrected for hand dominance. Per subgroup, the number of patients whose outcome scores exceeded these MCID thresholds were ascertained. These numbers were then converted into a percentage of the total number of persons within a subgroup.

In order to assess whether data were normally distributed or not, multiple Shapiro-Wilk tests were performed. As, eventually, nearly all data turned out to be non-normally distributed, data were statistically analysed using non-parametric statistics. Alpha was set at 0.05. The 'intention-to-treat' principle was used, i.e. patients were analysed according to the program in which they originally started. Friedman two-way analysis of variance by ranks was used for the data of each subgroup to determine whether patients improved over time, i.e. between all measurement dates from $T_{B L}$ up till $T_{12 m}$ regarding the FMA, grip strength, ARAT, and ABILHAND. Subsequent multiple comparisons between $T_{B L}$ and $T_{C D}$ data (to ascertain any changes in AHF and AHSP during the rehabilitation phase) as well as between $\mathrm{T}_{\mathrm{CD}}$ and $\mathrm{T}_{12 \mathrm{M}}$ data (representing changes the one year follow-up), included Wilcoxon signed ranks tests. A Bonferroni approach was used to control for spurious false positive findings.

For ascertaining any possible differences between subgroups as to the rate of improvement in arm-hand performance during rehabilitation and during the 12 months follow-up, Kruskal-Wallis tests were applied. Here, multiple comparisons included 
Mann-Whitney U-tests in a Bonferroni approach. Data were analysed using SPSS software (version 23.0, IBM Corp., New York).

\section{Results}

\section{Error analysis}

In total 89 patients entered the study. Three patients dropped out due to a recurring stroke during the study. Two patients prematurely left the study after the $T_{C D}$ measurement because of personal reasons. Six patient cases were discarded following the procedures we used regarding missing values (rule number 4), i.e. when data from three or more measurement dates were missing. No significant differences in patient characteristics existed between the patients who dropped out and the remaining patients.

Shapiro-Wilk tests revealed that the majority of data were not normally distributed. Therefore, data were analyzed using non-parametric statistics as described earlier.

\section{Patient characteristics}

The characteristics of the 89 patients included in the study are shown in Table 1. 
Table 1. Patient characteristics upon inclusion in the study.

\begin{tabular}{|c|c|c|c|c|}
\hline \multirow{2}{*}{ Characteristics } & \multirow{2}{*}{$\begin{array}{l}\text { Whole } \\
\text { group }\end{array}$} & \multicolumn{3}{|c|}{ Subgroups } \\
\hline & & 1 & 2 & 3 \\
\hline Total number $(\mathrm{n})$ & 89 & 28 & 28 & 33 \\
\hline Age (y), mean (sd) & $57.6(10.6)$ & $56.2(11.0)$ & $57.9(12.5)$ & $58.5(8.5)$ \\
\hline \multicolumn{5}{|l|}{ Gender (n, \%) } \\
\hline Male & $63(70.8 \%)$ & $15(53.6 \%)$ & $24(85.7 \%)$ & $24(72.7 \%)$ \\
\hline Female & $26(29.2 \%)$ & $13(46.4 \%)$ & $4(14.3 \%)$ & $9(27.3 \%)$ \\
\hline \multicolumn{5}{|l|}{ Side of lesion $(n, \%)$} \\
\hline Left & $53(59.6 \%)$ & $13(46.4 \%)$ & $18(64.3 \%)$ & $22(66.7 \%)$ \\
\hline Right & $36(40.4 \%)$ & $15(53.6 \%)$ & $10(35.7 \%)$ & $11(33.3 \%)$ \\
\hline \multicolumn{5}{|l|}{ Stroke Type (n, \%) } \\
\hline Haemorrhagic & $17(19.1 \%)$ & $5(17.9 \%)$ & $5(17.9 \%)$ & $7(21.2 \%)$ \\
\hline Ischemic & $72(80.9 \%)$ & $23(82.1 \%)$ & $23(82.1 \%)$ & $26(78.8 \%)$ \\
\hline \multicolumn{5}{|l|}{ Lesion site as diagnosed (n) } \\
\hline Basal ganglia & 7 & 1 & 2 & 4 \\
\hline Brainstem & 2 & & & 2 \\
\hline Capsula interna & 1 & & & 1 \\
\hline Cerebellum & 2 & & & 2 \\
\hline Frontal area & 2 & & 1 & 1 \\
\hline Frontoparietal area & 1 & 1 & & \\
\hline Frontotemporal area & 2 & 1 & 1 & \\
\hline Parietal area & 1 & & & 1 \\
\hline Parietotemporal area & 1 & 1 & & \\
\hline Posterior area & 1 & & & 1 \\
\hline Temporal area & 1 & & & 1 \\
\hline Temporal area $\&$ thalamus & 1 & & & 1 \\
\hline Thalamus & 4 & 1 & 1 & 2 \\
\hline Pontine & 1 & & & 1 \\
\hline Hemisphere & 54 & 22 & 20 & 12 \\
\hline Lacunar & 5 & & 3 & 2 \\
\hline Medulla oblong. \& cerebellum & 1 & & & 1 \\
\hline Nucleus caudatus & 2 & 1 & & 1 \\
\hline $\begin{array}{l}\text { Time post stroke (days), } \\
\text { mean (sd) }\end{array}$ & $29.8(20.1)$ & $40(27.5)$ & $27(14.5)$ & $23.4(12.6)$ \\
\hline \multicolumn{5}{|l|}{ Affected hand (n) } \\
\hline Dominant & 50 & 11 & 17 & 22 \\
\hline Non-dominant & 39 & 17 & 11 & 11 \\
\hline
\end{tabular}

$n=$ number; $s d=$ standard deviation . 


\section{Improvement over time within each subgroup}

\section{Fugl-Meyer Motor Assessment}

In Figure 2A - C the boxplots of the FMA data per measurement moment for each of the three groups are presented.
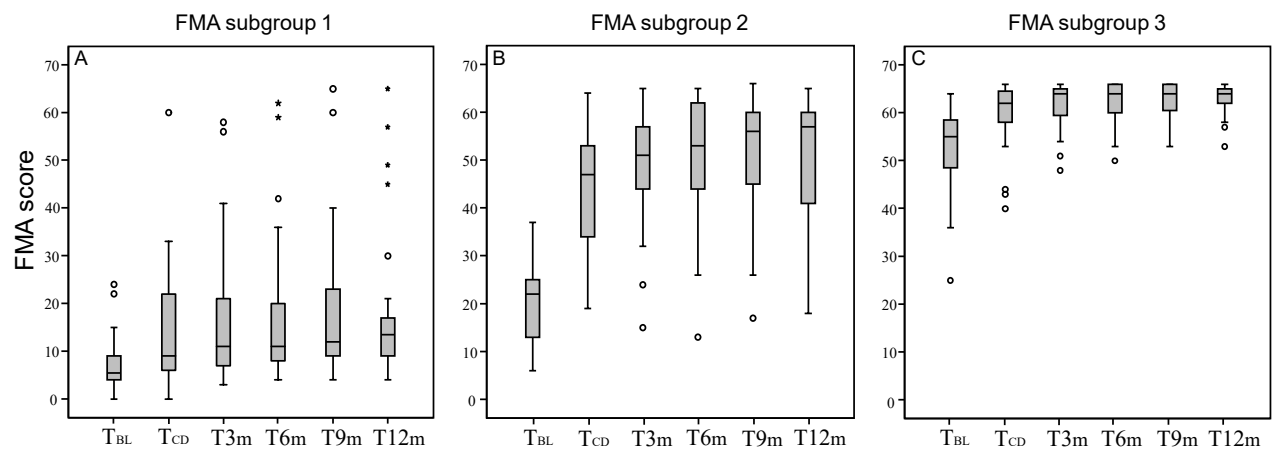

Figure 2. Boxplots of the FMA data per measurement moment for each of the three groups.

$F M A=$ Fugl Meyer Motor Assessment; $T_{B L}=$ Baseline; $T_{C D}=$ clinical discharge; $T_{3 m}=\left(=T_{C D}+3\right.$ months $) ; T_{6 m}\left(=T_{C D}\right.$

+ 6 months); $T_{9 m}\left(=T_{C D}+9\right.$ months); $T_{12 m}\left(=T_{C D}+12\right.$ months). Circle = outlier value; Asterisk = far out value.

Overall, patients of the three subgroups improved over time between $T_{B L}$ and $T_{12 m}$ on the FMA $(p<=0.000)$. Multiple comparison analyses revealed that in subgroup 1 the FMA improved between $T_{B L}$ and $T_{C D}(p=0.003)$ and between $T_{C D}$ and $T_{12 m}(p=0.009)$. In subgroup 2 significant improvements between $T_{B L}$ and $T_{C D}(p<=0.000)$ and between $T_{C D}$ and $T_{12 m}(p=0.004)$ were found. Similarly, in subgroup 3 improvements between $T_{B L}$ and $T_{C D}(p<=0.000)$ and $T_{C D}$ and $T_{12 m}(p=0.002)$ were found regarding the FMA scores.

\section{Grip strength}

In Figures 3A - C the boxplots of the grip strength data per measurement moment for each of the three groups are presented. 

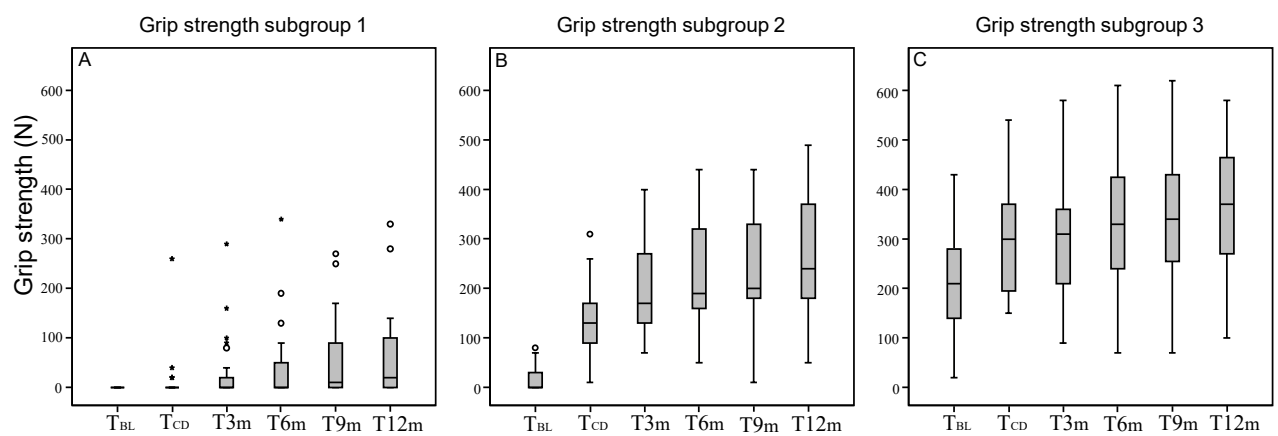

Figure 3. Boxplots of the grip strength data per measurement moment for each of the three groups.

$T_{B L}=$ Baseline; $T_{C D}=$ Clinical Discharge; $T_{3 m}=\left(=T_{C D}+3\right.$ months $) ; T_{6 m}\left(=T_{C D}+6\right.$ months $) ; T_{9 m}\left(=T_{C D}+9\right.$ months); $T_{12 m}\left(=T_{C D}+12\right.$ months). Circles = outlier value; Asterisk = far out value.

In general, patients of the three subgroups improved on grip strength between $T_{B L}$ and $T_{12 m}(p<=0.000)$. In subgroup 1 no significant changes between $T_{B L}$ and $T_{C D}(p=0.066)$ were found, in contrast to the follow-up period, i.e. between $T_{C D}$ and $T_{12 m}$, in which substantial improvements were found $(p=0.001)$. Subgroup 2 and subgroup 3 showed a significant improvement as to grip strength between $T_{B L}$ and $T_{C D}(p<=0.000)$, and, similarly, in the follow-up period between $T_{C D}$ and $T_{12 m}(p<=0.000)$.

\section{Action Research Arm Test}

In Figures 4A - $C$ the boxplots of the ARAT data per measurement moment for each of the three groups are presented.
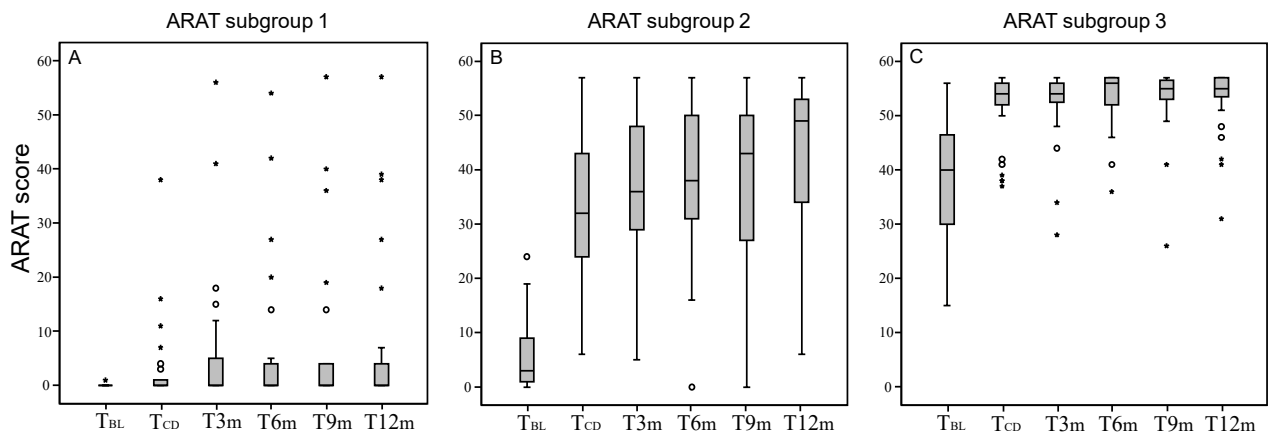

Figure 4. Boxplots of the ARAT data per measurement moment for each of the three groups.

ARAT = Action Research Arm Test; $T_{B L}=$ Baseline; $T_{C D}=$ Clinical Discharge; $T_{3 m}=\left(=T_{C D}+3\right.$ months $) ; T_{6 m}\left(=T_{C D}+\right.$ 6 months); $T_{9 m}\left(=T_{C D}+9\right.$ months); $T_{12 m}\left(=T_{C D}+12\right.$ months). Circles = outlier value; Asterisk = far out value.

Overall, patients of the three subgroups improved on the ARAT over time between $T_{B L}$ and 
$T_{12 m}(p<=0.000)$. Subgroup 1 progressed on the ARAT between $T_{B L}$ and $T_{C D}(p=0.018)$ and in the follow-up period between $T_{C D}$ and $T_{12 m}(p=0.007)$. In subgroup 2 progression was found between $T_{B L}$ and $T_{C D}(P<=0.000)$, and between $T_{C D}$ and $T_{12 m}(p=0.001)$. Subgroup 3 improved on the ARAT between $T_{B L}$ and $T_{C D}(p<=0.000)$. However, in the latter group, no significant changes on the ARAT were found between $T_{C D}$ and $T_{12 m}$ $(p=0.071)$.

\section{ABILHAND}

In Figures $5 \mathrm{~A}$ - $\mathrm{C}$ the boxplots of the ABILHAND data per measurement moment for each of the three groups are presented.
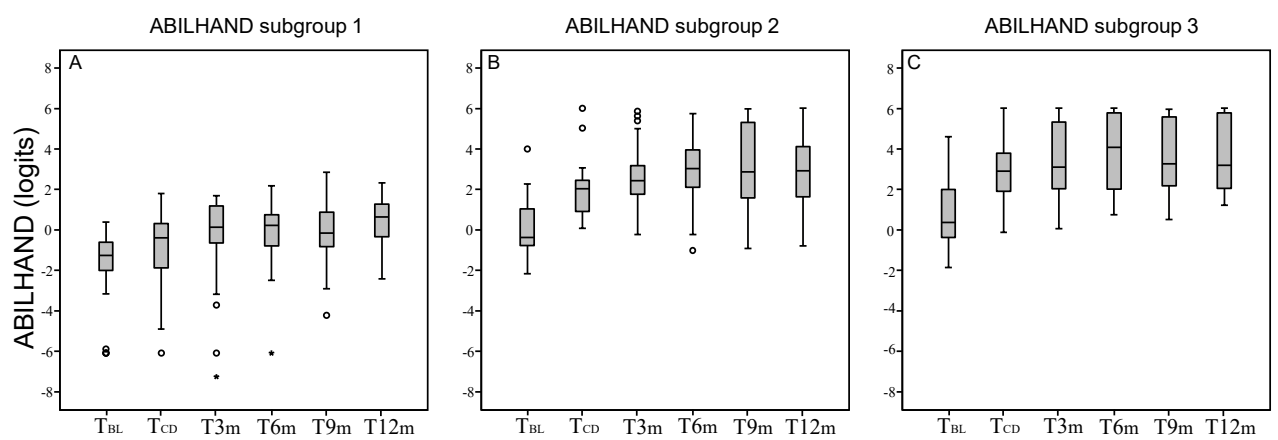

Figure 5. Boxplots of the ABILHAND results per measurement moment for each of the three groups. $T_{B L}=$ Baseline; $T_{C D}=$ Clinical Discharge; $T_{3 m}=\left(=T_{C D}+3\right.$ months $) ; T_{6 m}\left(=T_{C D}+6\right.$ months $) ; T_{9 m}\left(=T_{C D}+9\right.$ months $) ; T_{12 m}$ $\left(=T_{C D}+12\right.$ months $)$. Circles = outlier value; Asterisk = far out value.

Generally speaking, patients of subgroup 1, 2 and 3 improved on the ABILHAND over time $(p<=0.000$ ). Subgroup 1 significantly progressed on the ABILHAND between TBL and $T_{C D}(p=0.014)$, and between $T_{C D}$ and $T_{12 m}(p<=0.000)$. In subgroup 2 improvements on the ABILHAND were found between $T_{B L}$ and $T_{C D}(p<=0.000)$, but not between TCD and $T_{12 m}(p=0.044)$. Subgroup 3 improved on the ABILHAND between TBL and TCD $(p<=0.000)$, but not between $T_{C D}$ and $T_{12 m}(p=0.040)$.

\section{Deterioration of arm-hand function, capacity and performance post-rehabilitation}

In Table 2 the number and percentage of patients per subgroup whose arm-hand function, arm-hand capacity and/or performance either deteriorated (more than 5\% relative to clinical discharge performance), or remained equal or improved during the 
post-rehabilitation phase, are presented. Deterioration of arm-hand function, as measured with the FMA, occurred in $19.2 \%$ of the persons with a severely affected arm-hand. In persons with a moderately or mildly impaired arm-hand this occurred in $19.0 \%$ and $3.2 \%$, respectively. Deterioration of grip strength occurred in $0 \%$ of the severely affected subgroup patients, in $9.5 \%$ of the moderately impaired subgroup and in $3.2 \%$ of the mildly affected subgroup patients. Arm-hand capacity, as measured with ARAT, showed a deterioration of $0 \%$ in the severely affected group, $9.5 \%$ in the moderately affected subgroup and $3.2 \%$ in the mildly affected subgroup. Deterioration of perceived performance, as measured with the ABILHAND, occurred in $56.0 \%$ of persons with a severely affected arm-hand, in $23.8 \%$ and $29.0 \%$ of the moderately and mildly impaired persons, respectively.

Table 2. Number (and \%) of patients per subgroup whose arm-hand capacity and/or performance either deteriorated (more than $5 \%$ relative to clinical discharge performance), or remained equal or improved during the post-rehabilitation phase

\begin{tabular}{|c|c|c|c|c|}
\hline \multirow{3}{*}{ Test } & \multicolumn{2}{|l|}{ Worse or } & \multicolumn{2}{|l|}{ Subgroups } \\
\hline & Equal / & 1 & & \\
\hline & better & $(n=26)$ & $(n=21)$ & $(n=31)$ \\
\hline \multicolumn{5}{|l|}{ FMA } \\
\hline & worse & $5(19.2 \%)$ & $4(19.0 \%)$ & $1(3.2 \%)$ \\
\hline & equal / better & $21(80.8 \%)$ & $17(81.0 \%)$ & $30(96.8 \%)$ \\
\hline \multicolumn{5}{|c|}{ Grip strength } \\
\hline & worse & $0(0.0 \%)$ & $2(9.5 \%)$ & $1(3.2 \%)$ \\
\hline & equal / better & $26(100 \%)$ & $19(90.5 \%)$ & $30(96.8 \%)$ \\
\hline \multicolumn{5}{|c|}{ ARAT } \\
\hline & worse & $0(0.0 \%)$ & $2(9.5 \%)$ & $1(3.2 \%)$ \\
\hline & equal / better & $26(100 \%)$ & $19(90.5 \%)$ & $30(96.8 \%)$ \\
\hline \multicolumn{5}{|c|}{ ABILHAND } \\
\hline & worse & $14(53.8 \%)$ & $5(23.8 \%)$ & $9(29.0 \%)$ \\
\hline & equal / better & *11 (46.2\%) & $16(76.2 \%)$ & $22(71.0 \%)$ \\
\hline
\end{tabular}

FMA = Fugl Meyer Motor Assessment; ARAT = Action Research Arm Test; Asterisk = one data point in 1 patient (P10) regarding the ABILHAND is missing for TCd. 


\section{Differences in rate of improvement over time between subgroups}

In Table 3 the rates of improvement over time of subgroups regarding the FMA, grip strength, ARAT and ABILHAND are presented.

\section{Fugl-Meyer Motor Assessment}

Overall, the rate of improvement on the FMA between $T_{B L}$ and $T_{C D}$ differed between groups $(p<=0.000)$. Furthermore, FMA rate of improvement between $T_{B L}$ and $T_{C D}$ differed between group 1 and $2(p<=0.000)$, between group 2 and $3(p<=0.000)$ but not between group 1 and $3(p=0.064)$.

In general, no significant differences between groups regarding the rate at which FMA scores changed between $T_{C D}$ and $T_{12 m}$ were found $(p=0.177)$.

\section{Grip strength}

Overall, changes in grip strength between $T_{B L}$ and $T_{C D}$ differed between the three subgroups $(p<=0.000)$. More specifically, the rate of grip strength improvement differed significantly between group 1 and $2(p<=0.000)$, group 2 and $3(p=0.029)$ and between group 1 and $3(p<=0.000)$.

With respect to the $T_{C D}$ and $T_{12 m}$ measurement period, overall, differences as to the rate at which grip strength improved were found between the groups $(p=0.009)$. Multiple comparisons revealed a significant difference in the rate of improvement between group 1 and $2(p=0.005)$. However, no significant differences in the rate of improvement on grip strength were found between group 1 and $3(p=0.050)$ or group 2 and $3(p=0.078)$

\section{Action Research Arm Test}

Overall, the rate at which the ARAT improved between $T_{B L}$ and TCD differed among the three groups $(p<=0.000)$. Multiple comparisons revealed these differences to be present between all (three) combinations of subgroups $(p<=0.000)$.

Also, overall significant differences between the rate of improvement on the ARAT were found between the three subgroups $(p=0.004)$ regarding $T_{C D}$ and $T_{12 m}$. Further analysis showed statistically significant differences in improvement on the ARAT 
between group 1 and $2(p=0.008)$, group 2 and $3(p=0.001)$, but not between group 1 and $3(p=0.684)$.

\section{ABILHAND}

Overall, differences in the rate at which patients improved on the ABILHAND between $T_{B L}$ and $T_{C D}$ were not statistically significant between groups $(p=0.056)$. Regarding the $T_{C D}$ and $T_{12 m}$ follow-up phase, patients from the three subgroups also did not significantly differ as to their rate of improvement on the ABILHAND over time $(p=0.567)$. 


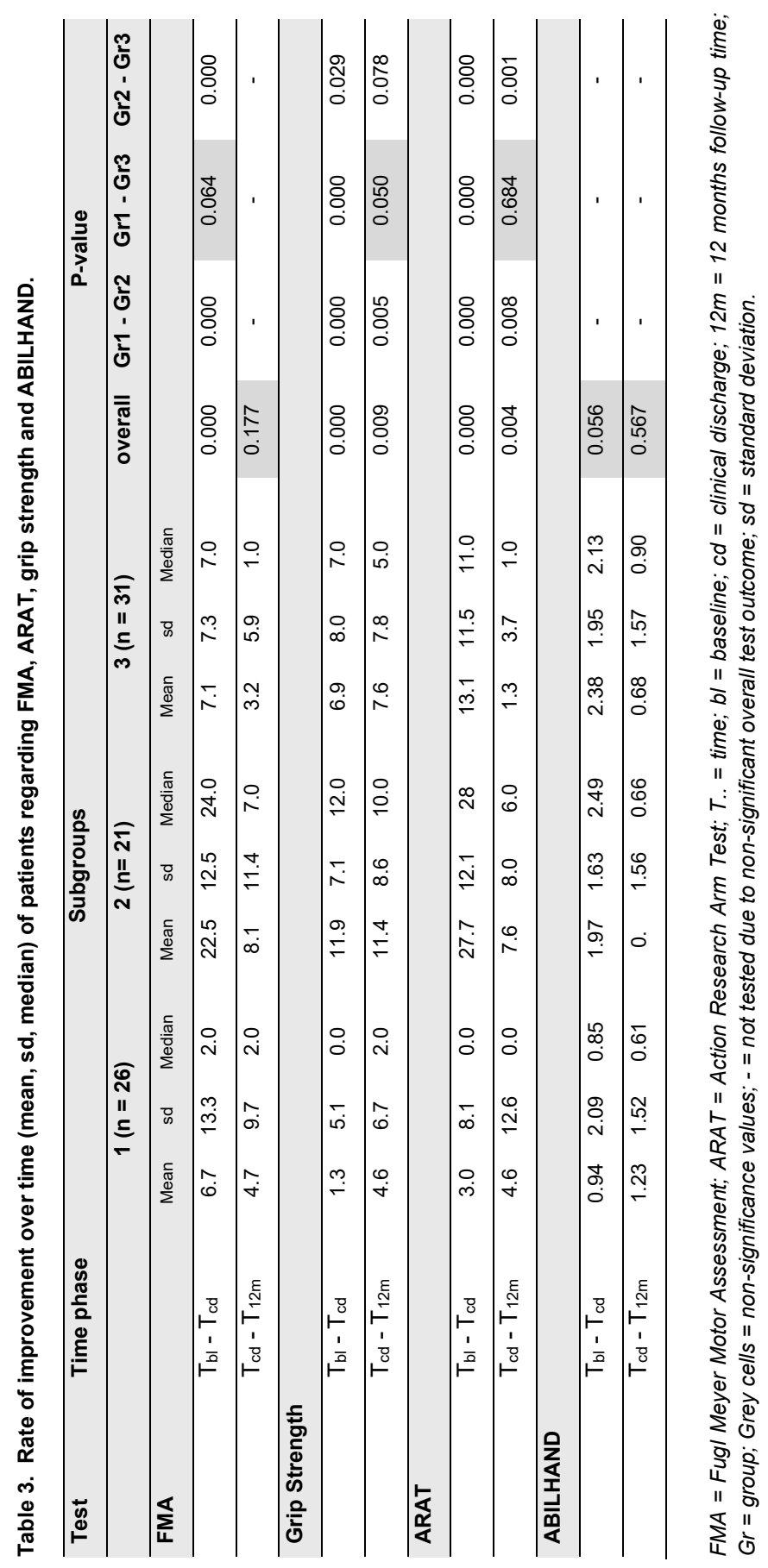




\section{Percentages of patients whose outcome scores exceeded the MCID thresholds}

In Table 4, for the FMA, grip strength, ARAT and ABILHAND, the number of patients whose outcome scores exceeded the MCID thresholds, expressed as the percentage of the total number of persons within each subgroup, are presented.

Table 4: Number of patients whose outcome scores exceeded the MCID thresholds, expressed as the percentage of the total number of persons within a subgroup, for FMA, grip strength, ARAT and ABILHAND

\begin{tabular}{|c|c|c|c|c|}
\hline \multirow{2}{*}{ Test } & \multirow{2}{*}{ Time phase } & \multicolumn{3}{|c|}{ Subgroups } \\
\hline & & 1 & 2 & 3 \\
\hline \multirow[t]{2}{*}{ FMA } & $T_{b l}-T_{c d}$ & $28 \%$ & $86 \%$ & $84 \%$ \\
\hline & $T_{c d}-T_{12 m}$ & $16 \%$ & $38 \%$ & $16 \%$ \\
\hline \multirow[t]{2}{*}{ Grip strength } & $T_{b l}-T_{c d}$ & $4 \%$ & $81 \%$ & $61 \%$ \\
\hline & $T_{c d}-T_{12 m}$ & $38 \%$ & $76 \%$ & $68 \%$ \\
\hline \multirow[t]{2}{*}{ ARAT } & $T_{b l}-T_{c d}$ & $4 \%$ & $81 \%$ & $57 \%$ \\
\hline & $T_{c d}-T_{12 m}$ & $8 \%$ & $24 \%$ & $3 \%$ \\
\hline \multirow[t]{2}{*}{ ABILHAND } & $T_{b l}-T_{c d}$ & $66 \%$ & $81 \%$ & $97 \%$ \\
\hline & $T_{c d}-T_{12 m}$ & $66 \%$ & $62 \%$ & $65 \%$ \\
\hline
\end{tabular}

FMA = Fugl Meyer Motor Assessment; ARAT = Action Research Arm Test; $T .$. = time; bl = baseline; cd = clinical discharge; $12 m=12$ months follow-up time

\section{Discussion}

The aim of the present study was to investigate a) to what extent arm-hand function (AHF) and arm-hand skill performance (AHSP) in subacute stroke patients change during and after their rehabilitation involving a well-described 'therapy-as-usual', and b) to what extent the rate of improvement or deterioration (over time) of AHF and AHSP differs between three subgroups of stroke patients. These patients are grouped according to their initial level of arm-hand impairment, i.e. severe (UAT 0-1), moderate (UAT 2-3) and mild (UAT 4-7) impairment. This study has been performed in a large 
stroke patient group typically seen in daily medical rehabilitation practice, i.e. only few inclusion and exclusion criteria were used, covering a broad spectrum of arm-hand problem severity levels, thus enhancing clinical generalisability of our results.

\section{Within-group results}

With respect to the first research question: On average, patients in all three subgroups, i.e. suffering from either a severely, moderately or mildly impaired arm-hand, improved over time regarding their arm-hand function and capacity as measured with the FMA, JAMAR and ARAT. More specifically, both during and after the rehabilitation phase, improvement on arm-hand capacity was observed in all three subgroups, except for the grip strength in the severely impaired group, which remained low during the rehabilitation phase, but did improve slightly during the 12 months post-rehabilitation. Given the late onset of the latter, it is very unlikely that this improvement of grip strength was caused by spontaneous recovery, whereas an increase in using the affected hand in assistance during daily living activities might explain this finding. Alternatively, as seen in many cases, the slight improvement in grip strength in the low functional group may have been caused by upcoming associated reactions, i.e. spasticity, in a later phase after stroke. This slight improvement in grip strength can be used by patients in their daily hand performance, although in most cases the functional benefits are minimal due to the negative side effects of associated reactions limiting the working range of the arm and hand.

During the rehabilitation phase, patients with an initially mildly or moderately affected arm-hand (subgroup 2 and 3) improved considerably as to arm-hand capacity and armhand function. Both groups were able to maintain these high levels during the postrehabilitation phase. It should, however, be noted that, for both the FMA and ARAT, patient scores in the mildly impaired subgroup related to the post-rehabilitation phase converged towards the maximum of the scale, thus constituting ceiling effects. Whether or not some of these patients may have further improved their arm-hand function and capacity beyond the scales' ranges during the post-rehabilitation phase is unclear, especially given the fact that grip strength and perceived arm-hand performance did significantly increase in these patients during this phase. 
Remarkably, seven patients who were diagnosed with an UAT score of 0-1 at baseline improved considerably as to their arm-hand capacity during and after the rehabilitation phase. These patients appear to represent a clinically meaningful subpopulation, distinctly different to the majority of patients across the low functional subgroup. Also the significant progressions observed at AHF and AHSP level between $T_{B L}$ and $T_{C D}$ within the moderately impaired group (group 2 ) leads to further questions concerning possible compensation and recovery mechanisms occurring at brain level in the subacute phase post-stroke. Improvements made regarding voluntary movement during and after the rehabilitation phase may be related to a substantial recovery of the corticospinal tract [42]. FMA outcome (arm-hand part) is said to be associated with cortico-spinal tract integrity and may be used in predicting recovery from motor impairment after stroke $[43,44]$. The progressions measured with the FMA between $T_{B L}$ and $T_{C D}$ within this moderately impaired group suggests that a certain degree of recovery of the cortico-spinal tract may have taken place. They underpin the observation that especially persons who are classified as 'moderately impaired' may go through a considerable recovery process regarding their affected arm and hand .This means that patients who lack any dexterity, i.e. finger extension at the start of program 2, still have an ability to generate and maintain significant progressions in AHF and AHSP. In terms of optimization of personalized arm-hand treatment and outcome, assessment should be focused on early detection of these patients among the population of patients with an initially low-functioning arm-hand.

In order to identify persons who initially show no recovery of hand movements, transcranial magnetic stimulation (TMS) [45] and diffusion tensor imaging (DTI) [46] are upcoming techniques to accurately predict arm-hand recovery. These techniques could be especially useful in the early phase of the rehabilitation process in assisting in deciding on rehabilitation goals and concomitant therapies.

As to self-perceived AHSP measured with the ABILHAND, as gauged using the ' $5 \%$ threshold' criterion, on average, all three groups improved over time. Whereas the severely impaired subgroup improved during both the rehabilitation phase and the post-rehabilitation phase, patients with an initially moderately or mildly impaired armhand function improved only significantly during the rehabilitation phase. During the 
post rehabilitation phase they were, again on average, able to maintain this selfperceived ability level, but did not increase any further. However, a more in-depth analysis of the post-rehabilitation data revealed that in 5 to $14 \%$ of all cases selfperceived AHSP deteriorated between clinical discharge and one-year follow-up, especially in the low functional group. However, when using the minimally clinically important difference (MCID) criterion in evaluating the ABILHAND data, a somewhat higher percentage of patients from subgroup 1 indicate having improved post-clinically. The self-perceived deterioration of AHSP by these patients is not in line with the results from more objectively quantified performance measures on capacity (ARAT) and function level (grip strength), which, for the larger part, remained at the same level or improved during the post-rehabilitation phase. One should, however, keep in mind that both ARAT and grip strength at clinical discharge were still low in a number of patients, especially in the (initially) low functioning group, and thus may have constituted socalled 'floor effects'.

\section{Between-group results}

With respect to the second research question: As to the potential differences in the rate of improvement between subgroups, there seems to be a non-linear, inverse $\mathrm{U}$ shaped relation between the rate of improvement at the level of AHF and AHSP on the one hand, and the severity of the loss of arm-hand function directly post-stroke. The largest gains as to AHF and AHSP, both during and after the rehabilitation phase, are seen in patients with an initially moderately affected arm-hand due to a stroke (UAT score 2-3), which is consistent with studies reported by Winters [23], Prabhakaran [22], Duncan [47] and Mirbagheri [48]. However, one should keep in mind that the majority of the patients with a moderately impaired arm-hand (in contrast to persons with a severely or mild impaired arm-hand) received a second six weeks period of training.

Furthermore, in contrast to patients with a moderately affected arm-hand, patients with an initially severely (UAT score 0-1) or mildly (UAT score 4-7) affected arm-hand showed a limited rate of improvement regarding the FMA and ARAT. In the latter group, however, ceiling effects in these measures as mentioned previously, might be responsible for this finding. 
In contrast to the progressions made at function level and capacity level, no significant differences between the rate of improvement at self-perceived performance level between subgroups were found, neither during nor after the rehabilitation phase. Nevertheless, what becomes clear from our data is that, across all subgroups, changes in self-perceived performance regarding arm-hand skills do not correlate well with changes in a patient's arm-hand skill capacity. Regarding the patients whose selfperceived performance decreases, the specific question arises what may have caused this deterioration in the post-rehabilitation phase. The answer to this question may be two-fold. First, one could argue that some patients' frame of reference regarding their perception and cognitions as to their daily skill performance may (negatively) change over time. This seems plausible as patients, once they are back in their own home environment, face daily reality and daily routine and may have difficulties to cope with this. Once in post-rehabilitation, i.e. chronic phase, patients may undergo a growing awareness as to their (in)ability in arm-hand performance. However, this topic should be investigated in-depth before a more definitive explanation can be provided. Finally, the ABILHAND, gauges a patient's performance on 23 fixed (bi)manual skills, not all of which each patient will perform in his daily routines. However, in the rehabilitation phase the patient seems to perceive his own performance more positively, in contrast to the post-rehabilitation phase in which he experiences more difficulties in the daily life circumstances. This will inevitably yield low sub-scores on some skills being encoded as having become more difficult. A possible solution to this problem may be to further personalize the list of skills to better fit the patient's changing needs and skill ambitions across time, which currently is beyond the scope of the ABILHAND.

\section{Strength and limitations of this study}

Most evidence-based therapies that have been shown to be effective for arm-hand performance in the post-stroke phase, are based on the testing of a single experimental intervention relative to various shapes of 'therapy-as-usual' in a group of preselected patients with at least some residual arm-hand function, predominantly focussing on persons with a mildly affected arm and hand. However, research studying the performance of patients with an unfavourable prognosis and/or a non-functional hand (i.e. UAT score $0-1$ and $2-3$ ) are scarce in literature $[21,49,50]$. The present study was 
explicitly designed to monitor the development of arm-hand use and skill performance in a broad range of stroke patients across the full stroke severity range who received a well-described 'therapy-as-usual', i.e. CARAS [18]. Next to evidence that a large majority of patients improved as to their AHF and AHSP, our study also provides evidence that, in a minority of patients, AHF and self-perceived AHSP deteriorate once they have left the active clinical rehabilitation setting.

The latter especially holds for patients with an initially moderately or severely affected arm-hand function (subgroup 1 and 2).

With respect to the results achieved in all groups the following remarks have to be made. First, the majority of the patients with a moderately impaired arm-hand (in contrast to persons with a severely or mild impaired arm-hand) received a second six weeks period of training. This particular group of patients do have the possibility to use their affected hand again in daily performance. However, due to their moderately impaired arm-hand they need a second 6-week during period of training. Second, patients in program 1 spend 4.5 hours per week on training, while patients in program 2 and 3 spend six hours per week of training. When neurophysiological recovery is absent, patients may be left with a non-functional arm-hand, which cannot be used in daily activities. Therefore, from a clinical point of view it is not useful to train patients in program 1 (severely affected) according the same practice conditions and as intensively as patients in program 2 and 3 (i.e. moderately and mildly affected patients). In contemporary clinical trials investigating effects of new therapy approaches patients with a moderately to severely affected hand are very often excluded. It is our firm opinion that these patient sub-groups deserve more scientific research attention regarding the exploration of the possibilities in sensorimotor training methods, especially at an early stage post-stoke.

The frame of reference of patients regarding the outcomes at the level of self-perceived AHSP may be influenced because they may reflect on their arm-hand abilities as how they were previously before the stroke occurred. The use of proxy-measurement has been considered. However, proxy-measurement might lead to other difficulties. First, regarding perceived performance it is difficult to avoid unreliable outcomes from relatives who also are familiar with the patients' former AHSP level. Second, the 
measures as used in this study are not designed to be used as proxy-measurement instruments.

CARAS has not been proven to be superior to other arm-hand therapy approaches as described by e.g. Winstein [9], Combs [10], Arya [11], Wallace [12], McDonnell [13], Platz [14], Morris [15], and Harris [16]. As Pollock et al., [51] already highlighted, clinical decision making procedures and the clinical application of arm-hand interventions have to be tailored to the patient's individual needs. CARAS is a clearly defined 'therapy-asusual', which provides practical solutions based on the presence of dexterity and corresponding functional possibilities which facilitates a better focus and tailored therapy delivery. It provides instructions how to empower patients by using principles of self-efficacy, and allows for the systematic incorporation of (new) technology and new evidence-based training elements swiftly [18], specifically adapted to the severity of the arm-hand impairment.

\section{Conclusion}

The present study has yielded a comprehensive longitudinal database on the development of AHF and AHSP in a broad range of stroke patients suffering from armhand impairments, who received well-documented 'therapy-as-usual', which may be used in future research as a reference database to contrast newly developed training interventions.

\section{Acknowledgements}

We are grateful to all patients who participated in this study and to all therapists who contributed to this study. 


\section{References}

1. Wade DT, Wood VA, Hewer RL. Recovery after stroke--the first 3 months. Journal of Neurology Neurosurgery and Psychiatry. 1985;48(1):7-13.

2. Langhorne P, Bernhardt J, Kwakkel G. Stroke rehabilitation. Lancet. 2011;377:1693 - 702.

3. French B, Thomas LH, Leathley MJ, Sutton CJ, McAdam J, Forster A, et al. Repetitive Task Training for Improving Functional Ability After Stroke. Stroke. 2009 Feb 26. PubMed PMID: 19246714.

4. Verbunt JA, Seelen HA, Ramos FP, Michielsen BH, Wetzelaer WL, Moennekens M. Mental practice-based rehabilitation training to improve arm function and daily activity performance in stroke patients: a randomized clinical trial. BMC Neurol. 2008;8:7. PubMed PMID: 18405377.

5. Wolf SL, Winstein CJ, Miller JP, Thompson PA, Taub E, Uswatte G, et al. Retention of upper limb function in stroke survivors who have received constraint-induced movement therapy: the EXCITE randomised trial. Lancet Neurol. 2008 Jan;7(1):33-40. PubMed PMID: 18077218.

6. Timmermans AA, Seelen HA, Willmann RD, Bakx W, de Ruyter B, Lanfermann G, et al. Arm and hand skills: Training preferences after stroke. Disabil Rehabil. 2009 May 19;31(16):1344-52. PubMed PMID: 19479535.

7. Timmermans AA, Seelen HA, Willmann RD, Kingma H. Technology-assisted training of arm-hand skills in stroke: concepts on reacquisition of motor control and therapist guidelines for rehabilitation technology design. J Neuroeng Rehabil. 2009;6:1. PubMed PMID: 19154570.

8. Winstein CJ, Stewart JC. Textbook of Neural Repair and Rehabilitation Cambridge: Cambridge University Press; 2006. 89-102

9. Winstein CJ, Rose DK, Tan SM, Lewthwaite R, Chui HC, Azen SP. A randomized controlled comparison of upper-extremity rehabilitation strategies in acute stroke: A pilot study of immediate and long-term outcomes. Arch Phys Med Rehabil. 2004 Apr;85(4):6208. PubMed PMID: 15083439.

10. Combs SA, Kelly SP, Barton R, Ivaska M, Nowak K. Effects of an intensive, task-specific rehabilitation program for individuals with chronic stroke: a case series. Disabil Rehabil. 2010;32(8):669-78. PubMed PMID: 20205581.

11. Arya KN, Verma R, Garg RK, Sharma VP, Agarwal M, Aggarwal GG. Meaningful taskspecific training (MTST) for stroke rehabilitation: a randomized controlled trial. Top Stroke Rehabil. 2012 May-Jun;19(3):193-211. PubMed PMID: 22668675.

12. Wallace AC, Talelli P, Dileone M, Oliver R, Ward N, Cloud G, et al. Standardising the intensity of upper limb treatment in rehabilitation medicine. Clin Rehabil. 2010;24(5):471-8. 
13. McDonnell MN, Hillier SL, Miles TS, Thompson PD, Ridding MC. Influence of combined afferent stimulation and task-specific training following stroke: a pilot randomized controlled trial. Neurorehabil Neur Rep. 2007 Sep-Oct;21(5):435-43.

14. Platz T, Eickhof C, van Kaick S, Engel U, Pinkowski C, Kalok S, et al. Impairment-oriented training or Bobath therapy for severe arm paresis after stroke: a single-blind, multicentre randomized controlled trial. Clin Rehabil. 2005 Oct;19(7):714-24. PubMed PMID: 16250190.

15. Morris JH, van Wijck F, Joice S, Ogston SA, Cole I, MacWalter RS. A comparison of bilateral and unilateral upper-limb task training in early poststroke rehabilitation: a randomized controlled trial. Arch Phys Med Rehabil. 2008 Jul;89(7):1237-45. PubMed PMID: 18586126.

16. Harris JE, Eng JJ, Miller WC, Dawson AS. A self-administered Graded Repetitive Arm Supplementary Program (GRASP) improves arm function during inpatient stroke rehabilitation: a multi-site randomized controlled trial. Stroke. 2009 Jun;40(6):2123-8. PubMed PMID: 19359633.

17. Barker RN, Brauer SG. Upper limb recovery after stroke: the stroke survivors' perspective. Disabil Rehabil. 2005 Oct 30;27(20):1213-23. PubMed PMID: 16298923. Epub 2005/11/22. eng.

18. Franck JA, Halfens JAM, Smeets RJEM, Seelen HAM. Concise Arm and hand Rehabilitation Approach in Stroke (CARAS): A practical and evidence-based framework for clinical rehabilitation management. OJOT. 2015;3(4):Article 10.

19. Kruitwagen-van Reenen ET, Post MW, Mulder-Bouwens K, Visser-Meily JM. A simple bedside test for upper extremity impairment after stroke: validation of the Utrecht Arm/Hand Test. Disabil Rehabil. 2009;31(16):1338-43.

20. Lemmens RJ, Timmermans AA, Janssen-Potten YJ, Smeets RJ, Seelen HA. Valid and reliable instruments for arm-hand assessment at ICF activity level in persons with hemiplegia: a systematic review. BMC Neurol. 2012;12:21. PubMed PMID: 22498041. Pubmed Central PMCID: PMC3352056. Epub 2012/04/14. eng.

21. Hayward K, Barker R, Brauer S. Interventions to promote upper limb recovery in stroke survivors with severe paresis: a systematic review. Disabil Rehabil. 2010;32(24):1973-86. PubMed PMID: 20964563.

22. Prabhakaran S, Zarahn E, Riley C, Speizer A, Chong JY, Lazar RM, et al. Inter-individual variability in the capacity for motor recovery after ischemic stroke. Neurorehabil Neur Rep. 2008 Jan-Feb;22(1):64-71. PubMed PMID: 17687024. Epub 2007/08/10. eng. 
23. Winters C, van Wegen EE, Daffertshofer A, Kwakkel G. Generalizability of the Proportional Recovery Model for the Upper Extremity After an Ischemic Stroke. Neurorehabil Neur Rep. 2015 Aug;29(7):614-22. PubMed PMID: 25505223. Epub 2014/12/17. eng.

24. Broeks JG, Lankhorst GJ, Rumping K, Prevo AJ. The long-term outcome of arm function after stroke: results of a follow-up study. Disabil Rehabil. 1999 Aug;21(8):357-64. PubMed PMID: 10503976.

25. Lyle RC. A performance test for assessment of upper limb function in physical rehabilitation treatment and research. Int J Rehabil Res. 1981;4(4):483-92. PubMed PMID: 7333761.

26. Gladstone DJ, Danells CJ, Black SE. The fugl-meyer assessment of motor recovery after stroke: a critical review of its measurement properties. Neurorehabil Neur Rep. 2002 Sep;16(3):232-40. PubMed PMID: 12234086.

27. Salter K, Jutai JW, Teasell R, Foley NC, Bitensky J. Issues for selection of outcome measures in stroke rehabilitation: ICF Body Functions. Disabil Rehabil. 2005 Feb 18;27(4):191-207. PubMed PMID: 15824050.

28. Arya KN, Verma R, Garg RK. Estimating the Minimal Clinically Important Difference of an Upper Extremity Recovery Measure in Subacute Stroke Patients. Top Stroke Rehabil. 2011;18:599-610.

29. Hamilton GF, McDonald C, Chenier T. Meting van de grijpkracht: waarde en betrouwbaarheid van de bloeddrukmeter en de 'Jamar grijpdynamometer'. Stimulus. 1994; Volume 13(3):164-5.

30. Lang CE, Edwards DF, Birkenmeier RL, Dromerick AW. Estimating minimal clinically important differences of upper-extremity measures early after stroke. Arch Phys Med Rehabil. 2008 Sep;89(9):1693-700. PubMed PMID: 18760153.

31. Hsieh CL, Hsueh IP, Chiang FM, Lin PH. Inter-rater reliability and validity of the action research arm test in stroke patients. Age Ageing. 1998 Mar;27(2):107-13. PubMed PMID: 16296669

32. Weerdt de WHM. Measuring recovery of arm-hand function in stroke patients: a comparison of the Brunnstrom-Fugl-Meyer test and the Action Research Arm test. Physiother Can. 1985;37:65-70.

33. Yozbatiran N, Der-Yeghiaian L, Cramer SC. A standardized approach to performing the action research arm test. Neurorehabil Neur Rep. 2008 Jan-Feb;22(1):78-90. PubMed PMID: 17704352.

34. Van der Lee JH, De Groot V, Beckerman H, Wagenaar RC, Lankhorst GJ, Bouter LM. The intra- and interrater reliability of the action research arm test: a practical test of upper 
extremity function in patients with stroke. Arch Phys Med Rehabil. 2001 Jan;82(1):14-9. PubMed PMID: 11239280.

35. Ashford S, Slade M, Malaprade F, Turner-Stokes L. Evaluation of functional outcome measures for the hemiparetic upper limb: a systematic review. J Rehabil Med. 2008 Nov;40(10):787-95. PubMed PMID: 19242614.

36. Penta M, Tesio L, Arnould C, Zancan A, Thonnard JL. The ABILHAND questionnaire as a measure of manual ability in chronic stroke patients: Rasch-based validation and relationship to upper limb impairment. Stroke. 2001 Jul;32(7):1627-34. PubMed PMID: 11441211.

37. Penta M, Thonnard JL, Tesio L. ABILHAND: a Rasch-built measure of manual ability. Arch Phys Med Rehabil. 1998 Sep;79(9):1038-42. PubMed PMID: 9749680.

38. Wang TN, Lin KC, Wu CY, Chung CY, Pei YC, Teng YK. Validity, Responsiveness, and Clinically Important Difference of the ABILHAND Questionnaire in Patients With Stroke. Arch Phys Med Rehabil. 2011;92:1086 - 91.

39. Kwakkel G, van Peppen R, Wagenaar RC, Wood Dauphinee S, Richards C, Ashburn A, et al. Effects of augmented exercise therapy time after stroke: a meta-analysis. Stroke. 2004 Nov;35(11):2529-39. PubMed PMID: 15472114.

40. Kwakkel G, Wagenaar RC, Twisk JW, Lankhorst GJ, Koetsier JC. Intensity of leg and arm training after primary middle-cerebral-artery stroke: a randomised trial. Lancet. $1999 \mathrm{Jul}$ 17;354(9174):191-6. PubMed PMID: 10421300.

41. Twisk JWR. Applied longitudinal data analysis for epidemiology. A practical guide. Cambridge: Cambridge University Press; 2003.

42. Jang SH, Kim YH, Cho SH, Lee JH, Park JW, Kwon YH. Cortical reorganization induced by task-oriented training in chronic hemiplegic stroke patients. Neurorep. 2003 Jan 20;14(1):137-41. PubMed PMID: 12544845.

43. Sullivan KJ, Tilson JK, Cen SY, Rose DK, Hershberg J, Anita. C, et al. Fugl-Meyer Assessment of Sensorimotor Function After Stroke Standardized Training Procedure for Clinical Practice and Clinical Trials. Stroke. 2011;42:427-32.

44. Smania N, Paolucci S, Tinazzi M, Borghero A, Manganotti P, Antonio F, et al. Active finger extension: a simple movement predicting recovery of arm function in patients with acute stroke. Stroke. 2007;38:1088 - 90.

45. Harris-Love M, Chan E, Dromerick A, Cohen L. Neural Substrates of Motor Recovery in Severely Impaired Stroke Patients With Hand Paralysis. Neurorehabil Neur Rep. 2016;30(4):328-38.

46. Kwah L, Herbert R. Prediction of Walking and Arm Recovery after Stroke: A Critical Review. Brain sci. 2016;6(53):1-16. 
47. Duncan P, Studenski S, Richards L, Gollub S, Lai SM, Reker D, et al. Randomized clinical trial of therapeutic exercise in subacute stroke. Stroke. 2003 Sep;34(9):2173-80. PubMed PMID: 12920254.

48. Mirbagheri MM, Rymer WZ. Time-course of changes in arm impairment after stroke: variables predicting motor recovery over 12 months. Arch Phys Med Rehabil. 2008;89(8):1507-13.

49. Kwakkel G, Kollen B. Predicting activities after stroke. In: Dietz V, Ward N, editors. Oxford Textbook of Neurorehabilitation. Oxford: Oxford University Press; 2015.

50. Oujamaa L, Relave I, Froger J, Mottet D, Pelissier JY. Rehabilitation of arm function after stroke. Literature review. Ann Phys Rehabil Med. 2009 Apr;52(3):269-93. PubMed PMID: 19398398.

51. Pollock A, Farmer SE, Brady MC, Langhorne P, Mead GE, Mehrholz J, et al. Interventions for improving upper limb function after stroke. Cochrane Database Syst Rev. 2014;11:CD010820. PubMed PMID: 25387001. Epub 2014/11/12. eng. 



\title{
Chapter
}

\begin{abstract}
Changes in actual arm-hand use in stroke patients during and after clinical rehabilitation involving a welldefined arm-hand rehabilitation program:
\end{abstract}

A prospective cohort study

Johan Anton Franck, Rob Johannes Elise Marie Smeets, Henk Alexander Maria Seelen 


\section{Abstract}

Introduction: Improvement of arm-hand function and arm-hand skill performance in stroke patients is reported by many authors. However, therapy content often is poorly described, data on actual arm-hand use are scarce, and, as follow-up time often is very short, little information on patients' mid- and long-term progression is available. Also, outcome data mainly stem from either a general patient group, unstratified for the severity of arm-hand impairment, or a very specific patient group.

Objectives: To investigate to what extent the rate of improvement or deterioration of actual arm-hand use differs between stroke patients with either a severely, moderately or mildly affected arm-hand, during and after rehabilitation involving a well-defined rehabilitation program.

Methods: Design: single-armed prospective cohort study. Outcome measure: affected arm-hand use during daily tasks (accelerometry), expressed as 'Intensity-of arm-hand-use' and 'Duration-of-arm-hand-use' during waking hours. Measurement dates: at admission, clinical discharge and 3, 6, 9 and12 months post-discharge. Statistics:2-way repeated measures ANOVAs.

Results: Seventy-six patients (63 males); mean age: 57.6 years (sd:10.6); post-stroke time: 29.8 days (sd:20.1) participated. Between baseline and 1-year follow-up, Intensity-of-armhand-use on the affected side increased by $51 \%, 114 \%$ and $14 \%(p<.000)$ in the mildly, moderately and severely affected patients, respectively. Similarly, Duration-of-arm-handuse increased by $26 \%, 220 \%$ and $161 \%$ ( $p<.000)$. Regarding bimanual arm-hand use: Intensity-of-arm-hand-use increased by $44 \%, 74 \%$ and $30 \%(p<.000)$, whereas Durationof-arm-hand-use increased by $10 \%, 22 \%$ and $16 \%(p<.000)$.

Conclusion: Stroke survivors with a severely, moderately or mildly affected arm-hand showed different, though (clinically) important, improvements in actual arm-hand use during the rehabilitation phase. Intensity-of-arm-hand-use and Duration-of-arm-hand-use significantly improved in both unimanual and bimanual tasks/skills. These improvements were maintained until at least 1 year post-discharge. 


\section{Introduction}

After stroke, the majority of stroke survivors experiences significant arm-hand impairments $[1,2]$ and a decreased use of the paretic arm and hand in daily life [3]. The actual use of the affected hand in daily life performance depends on the severity of the arm-hand impairment [4-6] and is associated with perceived limitations in participation [7, 8]. Severity of arm-hand impairment is also associated with a decrease of health-related quality of life [9], restricted social participation [10], and subjective well-being [11, 12].

Numerous interventions and arm-hand rehabilitation programs have been developed in order to resolve arm-hand impairments in stroke patients $[6,13]$. In the Netherlands, a number of stroke units in rehabilitation centres implemented a well-described 'therapy-asusual' arm-hand rehabilitation program, called CARAS (acronym for: Concise Arm and hand Rehabilitation Approach in Stroke)[14], serving a broad spectrum of stroke patients across the full stroke severity range of arm-hand impairments. The arm-hand rehabilitation program has been developed to guide clinicians in systematically designing arm-hand rehabilitation, tailored towards the individual patient's characteristics while keeping control over the overall heterogeneity of this population typically seen in stroke rehabilitation centres. A vast majority of stroke patients who participated in CARAS improved on armhand function (AHF), on arm-hand skilled performance (AHSP) capacity and on (self-) perceived performance, both during and after clinical rehabilitation [15]. The term 'armhand function' (AHF) refers to the International Classification of Functioning (ICF) [16] 'body function and structures level'. The term 'arm-hand skilled performance' (AHSP) refers to the ICF 'activity level', covering capacity as well as both perceived performance and actual arm-hand use [17].

Improved AHF and/or AHSP capacity do not automatically lead to an increase in actual arm-hand use and do not guarantee an increase of performing functional activities in daily life [18-20]. Improvements at function level, i.e. regaining selectivity, (grip) strength and/or grip performance, do not automatically lead to improvements experienced in real life task performance of persons in the post-stroke phase who live at home [18, 21]. Next to outcome measures regarding AHF, AHSP capacity and (self-) perceived AHSP, which are typically measured in controlled conditions, objective assessment of functional activity and actual arm-hand use outside the testing situation is warranted [22, 23]. 
Accelerometry can be used to reliably and objectively assess actual arm-hand use during daily task performance [24-32] and has been used in several studies to detect arm-hand movements and evaluate arm-hand use in the post-stroke phase [20, 3335]. Previous studies have demonstrated that, in stroke patients, movement counts, as measured with accelerometers, are associated with the use of the affected arm-hand (Motor Activity Log score) [36, 37] and, at function level, with the Fugl-Meyer Motor Assessment [38]. Next to quantifying paretic arm-hand use, accelerometers have also been used to provide feedback to further enhance the use of the affected hand in home-based situations [39]. Most studies consist of relatively small [27, 30, 40-44] and highly selected study populations [45] with short time intervals between baseline and follow-up measurements. As to our knowledge, only a few studies monitored arm-hand use in stroke patients for a longer period, i.e. between time of discharge to a home situation or till 6 to 12 months after stroke $[19,44,46]$. However, they used a relatively small study sample and their intervention aimed at arm-hand rehabilitation was undefined. Both studies of Connell et al. and Uswatte et al. describe a well-defined arm hand intervention where accelerometry data were used as an outcome measure [27, 47]. However, the study population described by Connell et al. consisted of a relative small and a relative mildly impaired group of chronic stroke survivors. The study population described by Uswatte et al. consisted of a large group of sub-acute stroke patients within strict inclusion criteria ranges [37], who, due to significant spontaneous neurologic recovery within this sub-acute phase, had a mildly impaired arm and hand $[48,49]$. This means that the group lacked persons with a moderately to severely affected arm-hand, who are commonly treated in the daily rehabilitation setting.

The course of AHF and AHSP of a broad range of sub-acute stroke patients during and after rehabilitation involving a well-defined arm-hand rehabilitation program (i.e. CARAS) [14] has been reported by Franck et al. [15]. The present paper provides data concerning actual arm-hand use in the same study population, and focuses on two objectives. The first aim is to investigate changes in actual arm-hand use across time, i.e. during and after clinical rehabilitation, within a stroke patient group typically seen in daily medical rehabilitation practice, i.e. covering a broad spectrum of arm-hand problem severity levels, who followed a well-described arm-hand treatment regime. The second aim is to investigate to what extent improvement (or deterioration) regarding the use of the affected arm-hand in daily life situations differs between 
patient categories, i.e. patients with either a severely, moderately or mildly impaired arm-hand, during and after their rehabilitation, involving a well-defined arm-hand rehabilitation program.

\section{Methods}

\section{Design}

This study is a single-armed prospective cohort study conducted between February 2011 and May 2015. Stroke patients who experienced AHF impairments were assessed during and up till 12 months after their protocolled rehabilitation treatment. This study meets the principles as stated in the Declaration of Helsinki and was approved by the Medical Ethics Committee of Maastricht University Medical Centre in the Netherlands (dossier number NL35681.068.11).

Written informed consent was obtained from all participants prior to the start of their participation in this study.

\section{Population}

The study population consisted of a broad range of sub-acute stroke patients admitted to the inpatient stroke ward of Adelante Rehabilitation Centre in Hoensbroek, the Netherlands. Inclusion criteria were kept to a minimum, i.e.: age $\geq 18$ years; clinically diagnosed with central paresis of the arm/hand at entry in the study; ability to control sitting posture; a fair cognitive level, i.e. being able to understand the questionnaires and measurement instructions. Exclusion criteria were: additional complaints that may interfere with the execution of the measurements; no informed consent. 


\section{Procedures}

\section{Therapy-as-usual}

After having been admitted to the Concise Arm and hand Rehabilitation Approach in Stroke (CARAS) [14], all participants were coached in arm-hand training, how to cope with their affected hand and to (re-)explore how to use their arm-hand in daily life activities or, in case dexterity was lacking completely, how to learn to keep and maintain the affected arm-hand in an optimal condition. CARAS targets a broad spectrum of arm-hand impairments typically seen in a heterogeneous stroke rehabilitation population. This approach consists of a well-described program offering stepwise, transparent and comprehensible procedures, tailored to specific needs of the individual patient.

Based on the severity of arm-hand impairment, for which the Utrecht Arm-hand Test (UAT) was used [50], patients were stratified into three subgroups, i.e. subgroup 1 (severely affected arm-hand (UAT score 0-1)), subgroup 2 (moderately affected armhand (UAT score 2-3)), and subgroup 3 (mildly affected arm-hand (UAT score 4-7)). All patients followed one of three training programs within CARAS. Subgroup 1 followed program 1, titled 'taking care and prevention'. These patients are unable to use their affected arm and hand for skill performance in daily life situations (nonfunctional arm-hand). Program 1 contains different topics aimed at getting and keeping the affected shoulder and arm-hand in an optimal condition and learning strategies on what to do when discomfort arises. Patients in subgroup 2 (UAT score 2-3) were admitted to program 2, whereas patients in subgroup 3 (UAT score 4-7) followed program 3. Both program 2 and 3 incorporate (high) intensive, task-oriented training components aimed at optimal integration of the affected arm and hand in daily occupations. Patients in subgroup 2 work on passive and active stabilisation tasks in order to become able to use their affected arm and hand for, e.g., holding vegetables on a table while cutting them with a knife held in the non-affected hand. Patients from subgroup 3 relearn their abilities to use their affected arm and hand instantaneously in daily situations in which more complex (bi-)manual activities are necessary. Patients in program 1 spend 4.5 hours per week on training during a period of six weeks. Patients in program 2 spend six hours of training per week during 12 weeks and patients in program 3 spend six hours of training during each week for 6 weeks. In this 
study CARAS is considered as 'therapy-as-usual' i.e. the standard rehabilitation approach for patients who cope with arm-hand deficits due to stroke occurrence. A more detailed description of the therapy content and the basic assumptions of CARAS have been presented elsewhere by Franck and co-workers [14].

After baseline assessment, patients enrolled in one of the three programs and started training for six consecutive weeks. After six weeks the patient left the program and entered the second assessment. Progress made, was expressed in terms of functional goals reached, based on capacity and performance levels exceeding certain minimal clinically important thresholds, as captured by the outcome measures at function level and activity level. Depending on these results, it was possible for the patient to choose for a second (and final) six weeks period of training, which was then also evaluated [14].

\section{Outcome measures}

At the start of the study the following demographic data and characteristics of participants were recorded: Gender, age, time post-stroke, stroke localisation, affected side, hand dominance, and status of the affected arm and hand expressed in terms of dexterity (severely, moderately or mildly affected), based on the UAT score [50].

Every two weeks a short question was posed in order to establish the occurrence of any major event that may have prevented the participant from using one or both arms or hands. The questions posed, was: "Has there been any major problem during the last two weeks preventing you from using one or both hands? (yes/no). If so, please indicate (from a short list) which problem(s)."This information is important as it may be used in explaining changes in data trends due to non-therapy-related events (e.g. sudden reduction in arm-hand use because the patient became ill and was confined to bed).

At each measurement time point the amount of arm-hand use (on both the affected and non-affected side) was monitored for 3 consecutive days, using watch-like accelerometers

(Actiwatches (Actiwatch AW7, CamNtech, UK)). Accelerations were recorded and converted into digital signals with a sample frequency of $32 \mathrm{~Hz}$ and a quantization range between -128 and +128 . Accelerations between $-5 \mathrm{~g}$ and $+5 \mathrm{~g}$ can be recorded 
by the system. The threshold of movement detection (=system sensitivity) is $0.05 \mathrm{~g}$. [36]. Per measurement session, two Actiwatches had to be worn, one on each wrist. Both Actiwatches were held in place using a nylon strap on the wrist, i.e. at the distal part of the radius, with the device facing the dorsal side of the forearm.

\section{Measurement dates}

This study included six measurements. After admission to the rehabilitation centre and prior to the start in CARAS a baseline measurement $\left(T_{B L}\right)$ was performed, followed by a measurement at clinical discharge $\left(T_{C D}\right)$. After discharge, measurements were performed at four additional points in time, interspaced by three months: $T_{3 m}\left(=T_{C D}+3\right.$ months) through $T_{12 m}\left(=T_{C D}+12\right.$ months). Per measurement session, the participants wore both Actiwatches for a period of three consecutive days in which the amount of arm-hand use was recorded continuously. Figure 1 presents an overview of the measurement dates.

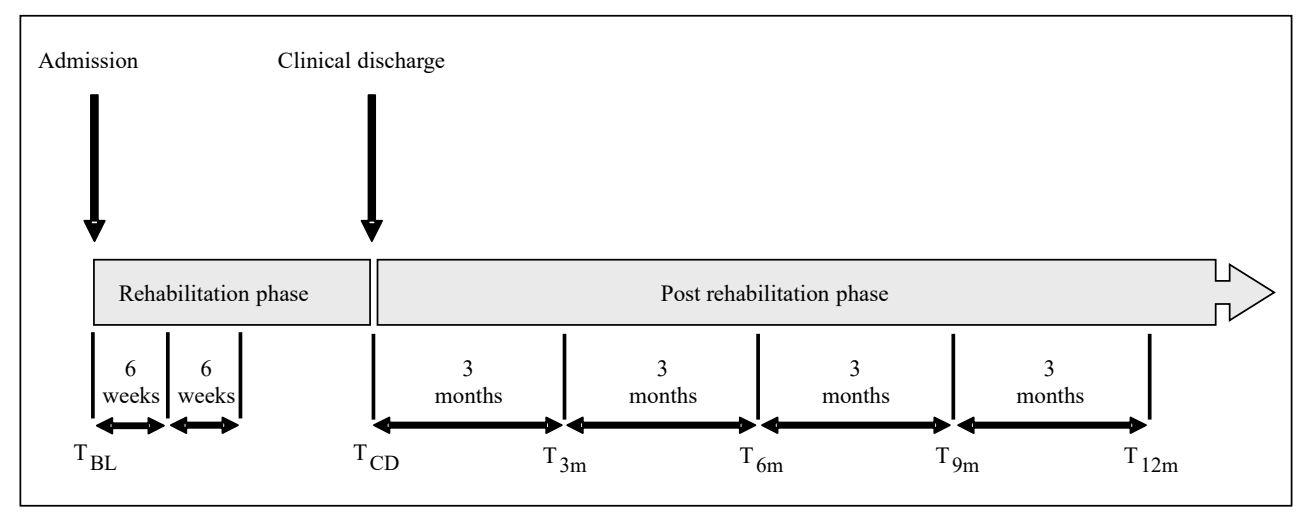

Figure 1. Overview of measurement timing. $T=$ time; $B L=$ baseline; $C D=$ clinical discharge; $M=$ month. 


\section{Signal processing}

Accelerations caused by arm-hand movement were recorded as analogue signals which were subsequently converted into a digital signal. The highest amplitude per 1second period, representing peak intensity, was registered as a "count". Every two seconds the two peak intensity values identified, were summed into a single number. This 2-second period was called an epoch [32, 51]. In this study the duration of the epoch was set per 2 seconds. This number then became a single data point in the final outcome signal or 'count' time series [36].

Figure 2 provides an example of a 'count' time series of three consecutive days of accelerometry data from a participant suffering from a paresis of the arm/hand.

Figure 2: Figure 2A represents data from the non-affected arm-hand, whereas Figure $2 \mathrm{~B}$ represents data from the affected arm-hand. Figure $2 \mathrm{C}$ represents the zero timelag low pass filtered signal of the non-affected arm-hand (dotted time series), the affected arm-hand (dashed-dotted time series) and the cumulative signal of the data of both hands (solid time series). 


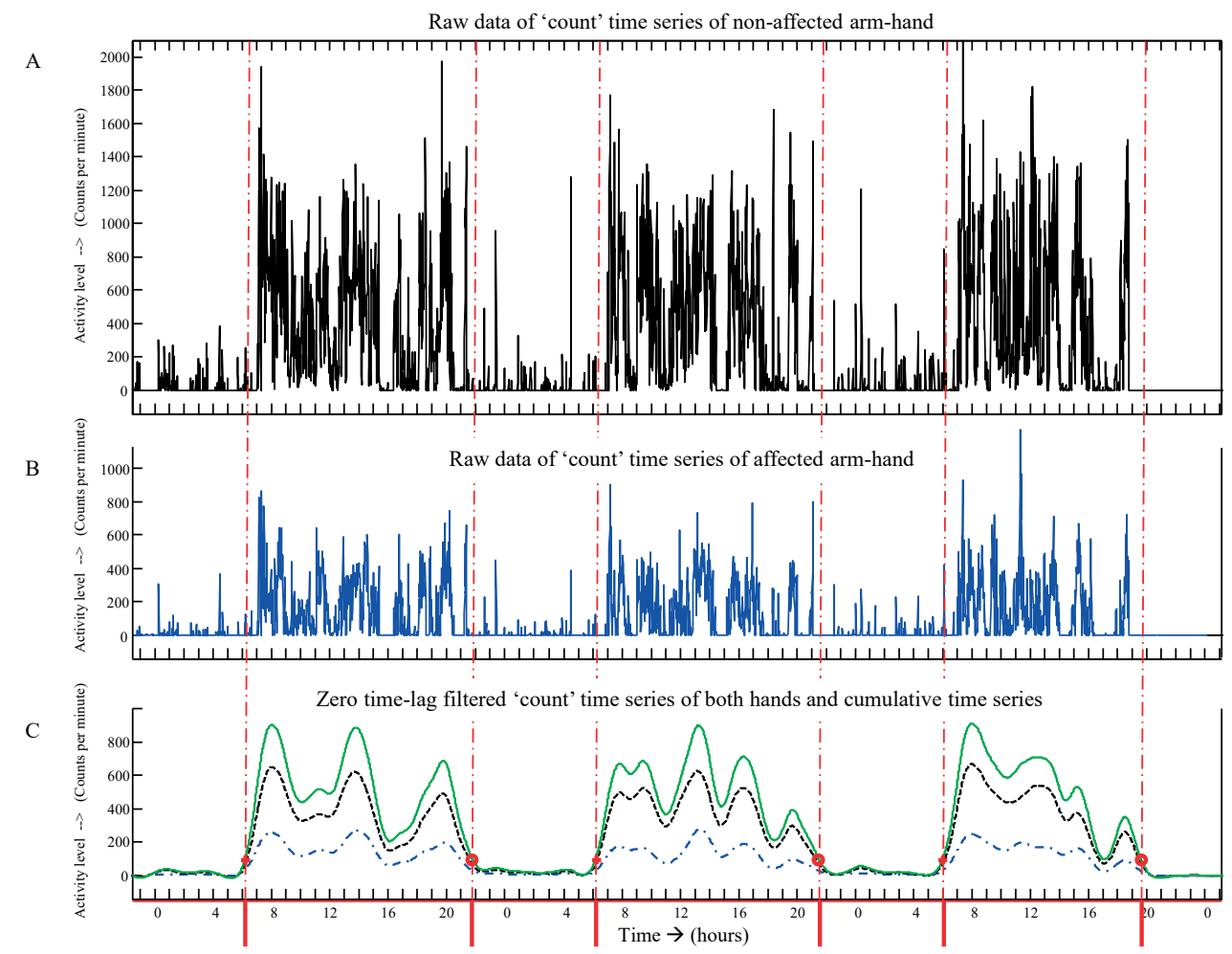

Figure 2. Accelerometer data 'count' time series of three consecutive days.

$A=$ 'count' time series from the non-affected arm; $B=$ count' time series from the affected arm; Dotted time series in Figure $2 \mathrm{C}=$ zero time-lag filtered signal from non-affected arm; Dashed-dotted time series in Figure $2 \mathrm{C}=$ zero time-lag filtered signal from affected arm; Solid time series in Figure $2 C=$ cumulative signal representing both filtered signals; vertical dashed-dotted lines = start and end of the so-called 'uptime' per day.

First, both 'count' time series, from the non-affected and the affected arm ('A' and 'B' respectively in Figure 2), were filtered using a zero time-lag second-order Butterworth filter (cut-off frequency: $0.0025 \mathrm{~Hz}$ ) (dotted time series and dashed-dotted time series in Figure 2C) and subsequently cumulated into one signal (solid line in Figure $2 \mathrm{C}$ ). From the latter signal, representing the arm-hand activity of both sides, the start and end of the so-called 'uptime' per day was identified (delimited by the vertical dashed-dotted lines on the time axis in Figure 2), using a predefined threshold. In order to avoid small resting periods during the uptime, in which neither arm-hand was used, to be falsely detected as 'night time', night time was a-priori defined as being longer than four consecutive hours. Minimal uptime length was defined as, at least, 10 consecutive hours per day. Night time data were discarded. 
Next, for each uptime, the average sum of 'counts' per minute was calculated for each of the two devices, i.e. for both arm-hands separately. This average sum of 'counts' per minute represents 'Intensity-of-arm-hand-use' of each arm-hand during the uptime period per minute [36, 51]. 'Duration-of-arm-hand-use' was calculated as the total amount of time (in seconds) in which there was activity of one or both hands exceeding a minimal threshold of signal intensity during the uptime.

Subsequently, more specific information on Intensity-of-arm-hand-use and on Durationof-arm-hand-use of both the affected and non-affected arm-hand was extracted from the 'count' time series data collected during uptime. First, the time (during uptime) in which the affected arm-hand was used unimanually, the time in which the non-affected arm-hand was used unimanually, the time both hands were used and the time none of the hands were used, was calculated, based on signal intensity crossing a predefined threshold. Next, for each of the aforementioned times the Intensity-of-arm-hand-use was calculated. Finally, in order to compare the Intensity-of-arm-hand-use of the affected arm-hand to that of the non-affected arm-hand, the ratio between the sum of counts of the affected arm (numerator) and the unaffected arm (denominator) was calculated. To avoid underestimation of the non-impaired arm-hand counts (denominator), compared to the impaired arm-hand (numerator), this ratio was log-transformed before (sub-)group averages were calculated [36]. Similarly, this procedure was done for the Duration-of-armhand-use data.

All accelerometry data were analyzed using MATLAB software version R2016a (The MathWorks Inc, Natick, MA, USA) and Microsoft Excel software version 2010 (Microsoft Corporation, Redmond, Washington, USA).

\section{Handling of missing values}

In order to handle missing data we used the following four decision rules, formulated prior to the start of the study;

1. When the baseline value was missing, this value was estimated using the mean baseline value of the subgroup the patient was allocated to.

2. When the $T_{12 m}$ value was missing, the 'last observation carried forward' procedure was used [52]. 
3. When 1 or 2 (temporally adjacent) value(s), not being the baseline value or the $\mathrm{T}_{12 \mathrm{~m}}$ value, were missing, these missing value(s) were estimated by linear interpolation using the two valid adjacent values in the time series.

4. In case of 3 or more missing values, the whole case was discarded.

\section{Statistical analyses}

The statistical analysis was performed using the intention-to-treat approach, following up all participants as originally allocated to a particular subgroup (1,2 or 3$)$ of CARAS. Statistical analyses included two-way repeated measures Analysis of Variance (ANOVA) (within-subject factor: Time, between-subject factor: AHF status). To test for normal distribution, the Shapiro-Wilk test was used [53]. Subsequently, multiple comparison was done using a Bonferroni approach to control for spurious false positive findings, involving two combinations of data sets, i.e. $T_{B L}$ vs. $T_{C D}$ and $T_{C D}$ vs. $T_{12 m}$, representing the clinical rehabilitation phase, and the post-rehabilitation phase, respectively. Alpha was set at 0.05 . Data were analyzed using SPSS software (version 24.0) (IBM Inc. Armonk, NY).

In the results section below, accelerometry data with respect to both Intensity-of-armhand-use and Duration-of arm-hand-use are reported in the following order: First, results regarding the affected arm-hand are presented. Second, the results of the use of the affected arm-hand during unimanual activities, are presented. Third, results regarding the use of both hands, while performing a task bimanually, are presented. And four, data regarding the ratio of Intensity-of-arm-hand-use of the affected hand and for both hands are presented.

\section{Results}

Patient characteristics and error analysis

A total of 89 patients entered the study. As a result of the application of the four previously stated decision rules, the data of 13 participants were discarded (rule number 4), leading to a remaining group of 76 participants used in the statistical analysis. For 16 (out of 380) measurement points, data were interpolated in accordance with rules 2 and 3 . 
Patient characteristics are presented in Table 1.

Table 1. Overview of patient characteristics at baseline.

\begin{tabular}{|c|c|c|c|c|}
\hline \multirow[t]{2}{*}{ Characteristics } & \multirow[t]{2}{*}{ Whole group } & \multicolumn{3}{|c|}{ Subgroups } \\
\hline & & Gr1 & Gr2 & Gr3 \\
\hline Total number: $(n)$ & 89 & 28 & 28 & 33 \\
\hline \multicolumn{5}{|l|}{ Age (years) } \\
\hline$($ mean $+/$-sd) & $57.6+/-10.6$ & $56.2+/-11.0$ & $57.9+/-12.5$ & $58.5+/-8.5$ \\
\hline \multicolumn{5}{|l|}{ Gender } \\
\hline - Male $\quad(n(\%))$ & $63 \quad(70.8 \%)$ & $15(53.6 \%)$ & $24 \quad(85.7 \%)$ & $24 \quad(72.7 \%)$ \\
\hline - Female (n (\%)) & $26 \quad(29.2 \%)$ & $13(46.4 \%)$ & $4 \quad(14.3 \%)$ & $9 \quad(27.3 \%)$ \\
\hline \multicolumn{5}{|l|}{ Lesion side } \\
\hline - Left (n (\%)) & $53 \quad(59.6 \%)$ & $13(46.4 \%)$ & $18(64.3 \%)$ & $22(66.7 \%)$ \\
\hline - Right (n (\%)) & $36 \quad(40.4 \%)$ & $15(53.6 \%)$ & $10(35.7 \%)$ & $11(33.3 \%)$ \\
\hline \multicolumn{5}{|l|}{ - Stroke Type } \\
\hline \multicolumn{5}{|l|}{ Haemorrhagic } \\
\hline$(n(\%))$ & $17 \quad(19.1 \%)$ & $5 \quad(17.9 \%)$ & $5 \quad(17.9 \%)$ & $7 \quad(21.2 \%)$ \\
\hline - Ischemic (n (\%)) & $72 \quad(80.9 \%)$ & $23 \quad(82.1 \%)$ & $23(82.1 \%)$ & $26 \quad(78.8 \%)$ \\
\hline $\begin{array}{l}\text { Time post stroke } \\
\text { (days) }(\text { mean }+/-s d)\end{array}$ & $29.8+/-20.1$ & $40+/-27.5$ & $27+/-14.5$ & $23.4+/-12.6$ \\
\hline \multicolumn{5}{|l|}{$\begin{array}{l}\text { - Affected hand: } \\
\text { dominant }(\mathrm{n}(\%))\end{array}$} \\
\hline & $50(56.2 \%)$ & $11(39.3 \%)$ & $17(60.7 \%)$ & $22(66.6 \%)$ \\
\hline $\begin{array}{l}- \text { non-dominant } \\
(\mathrm{n}(\%))\end{array}$ & $39(43.8 \%)$ & $17(60.7 \%)$ & $11(39.3 \%)$ & $11(33.3 \%)$ \\
\hline
\end{tabular}

Lesion site $(n)$

Basal ganglia (7), Brainstem (2), Capsula interna (1), Cerebellum (2), Frontal area (2), Frontoparietal area (1), Frontotemporal area (2), Parietal area (1), Parietotemporal area (1), Posterior area (1), Temporal area (1), Temporal area \& thalamus (1), Thalamus (4), Pontine (1), Hemispheric (not specified) (54), Lacunar (5), Medulla oblongata \& cerebellum (1), Nucleus caudatus (2). 


\section{CHAPTER 4}

\section{Intensity-of-arm-hand-use on the affected side during (overall) uptime}

Mean values for Intensity-of-arm-hand-use during (overall) uptime for subgroups 1, 2 and 3 are displayed in Figure 3. The corresponding statistics are presented in Table 2. From $T_{b l}$ across to $T_{12 m}$ main significant effects for 'Time', 'AHF status' and 'Time' $x$ 'AHF status' were found. Furthermore, whereas, on average, all 3 subgroups showed improvement between baseline and clinical discharge regarding Intensity-of-armhand-use on the affected side during uptime, participants from subgroup 2 seem to even further improve between discharge $\left(T_{C D}\right)$ and 12 months after clinical discharge ( $\mathrm{T}_{12 \mathrm{~m}}$ ). Participants admitted to subgroup 3 improved the most between baseline ( $\left.\mathrm{T}_{\mathrm{BL}}\right)$ and clinical discharge (TCD).

Intensity-of-arm-hand-use on the affected side

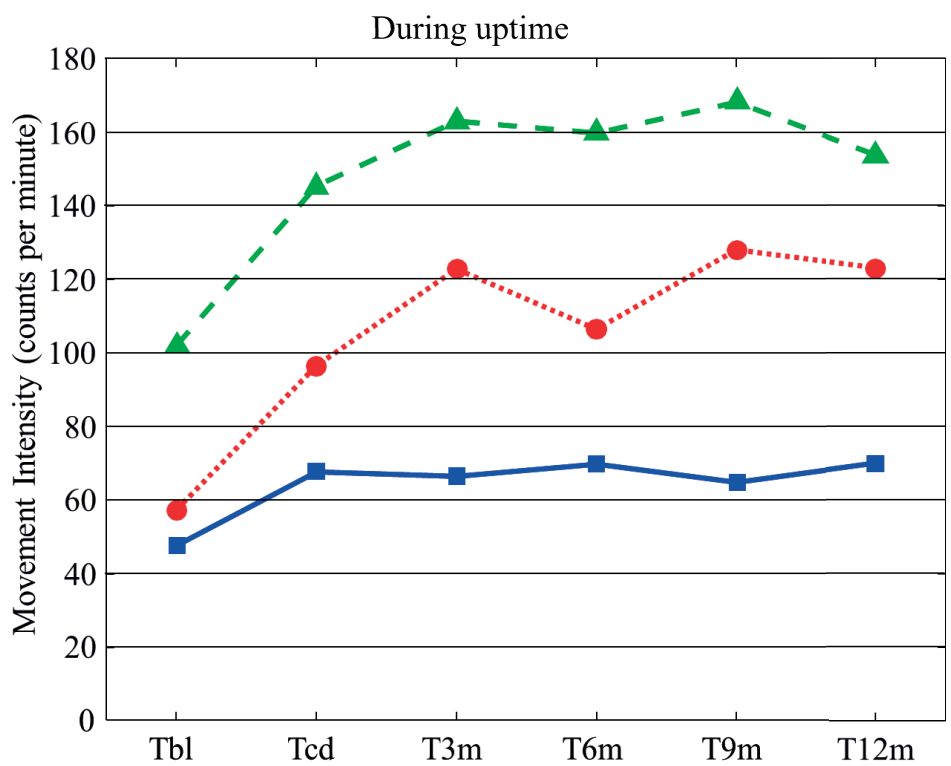

Figure 3. Mean values for Intensity-of-arm-hand-use during uptime for subgroups 1,2 and 3. T= time; bl= baseline; $c d=$ clinical discharge; $m=$ month; Solid line = subgroup 1; Dotted line = subgroup 2; Dashed line = subgroup 3. 
Intensity-of-arm-hand-use on the affected side during unimanual activities

Mean values for Intensity-of-arm-hand-use on the affected side during unimanual activities for subgroups 1,2 and 3 are displayed in Figure 4A. The corresponding statistics are presented in Table 2.

\section{Intensity-of-arm-hand-use on the affected side}
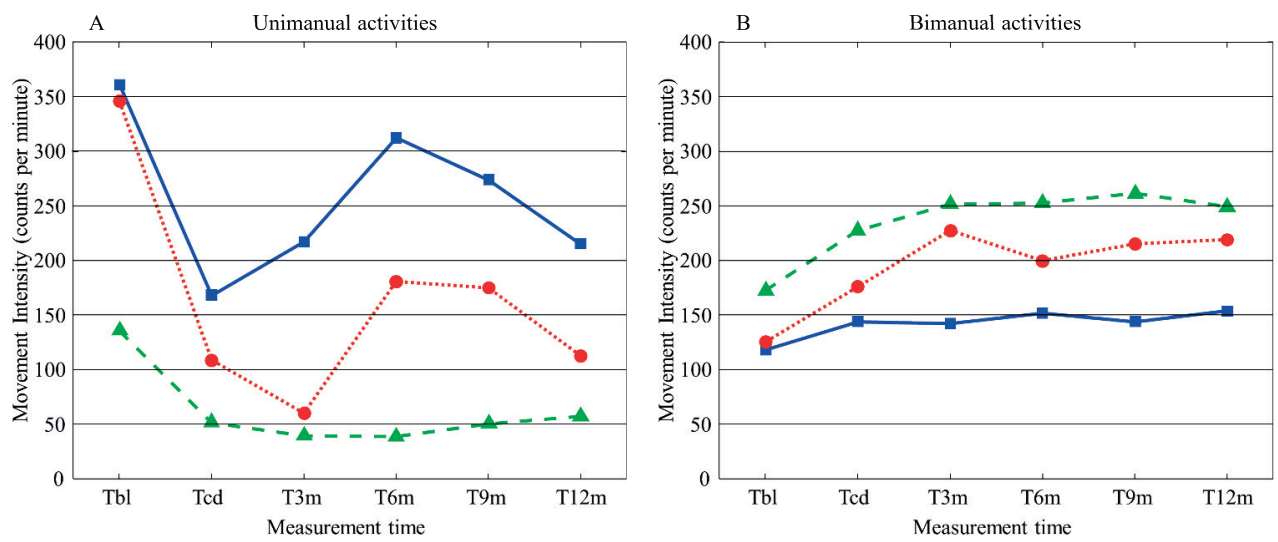

Figure 4A. Mean values for Intensity-of-arm-hand-use on the affected side during unimanual activities for subgroups 1, 2 and 3.

Figure 4B. Mean values for Intensity-of-arm-hand-use on the affected side during bimanual activities for subgroups 1, 2 and 3. $T=$ time; $b l=$ baseline; $c d=$ clinical discharge; $m=$ month. Solid line = subgroup 1; Dotted line = subgroup 2; Dashed line = subgroup 3.

The main findings were that, on average, the Intensity-of-arm-hand-use on the affected side during unimanual activities decreased in subgroup 1 and 2, and, although not statistically significant, to a lesser extent, in subgroup 3, between $T_{B L}$ and $T_{C D}$. Regarding results from $\mathrm{T}_{C D}$ and $\mathrm{T}_{12 \mathrm{~m}}$, no further change in Intensity-of-arm-hand-use on the affected side during unimanual activities was observed.

Intensity-of-arm-hand-use on the affected side during bimanual activities

Mean values for Intensity-of-arm-hand-use on the affected side during bimanual activities for subgroups 1, 2 and 3 are displayed in Figure 4B. The corresponding statistics are presented in Table 2. 
During bimanual task performance Intensity-of-arm-hand-use on the affected side improved in all three subgroups across time, i.e. between $T_{B L}$ and $T_{12 m}$. However, in subgroup 2 improvement between $\mathrm{T}_{\mathrm{BL}}$ and $\mathrm{T}_{12 \mathrm{~m}}$ was relatively larger compared to subgroup 1 and, though to a lesser extent, to subgroup 3. At baseline, participants from subgroup 2 used their affected arm-hand approximately equally to participants from subgroup 1 during bimanual activities.

\section{Duration-of-arm-hand-use on the affected side during unimanual activities}

Mean values for Duration-of-arm-hand-use on the affected side during unimanual activities for subgroups 1,2 and 3 are displayed in Figure 5A. The corresponding statistics are presented in Table 3.

Duration-of-arm-hand-use on the affected side
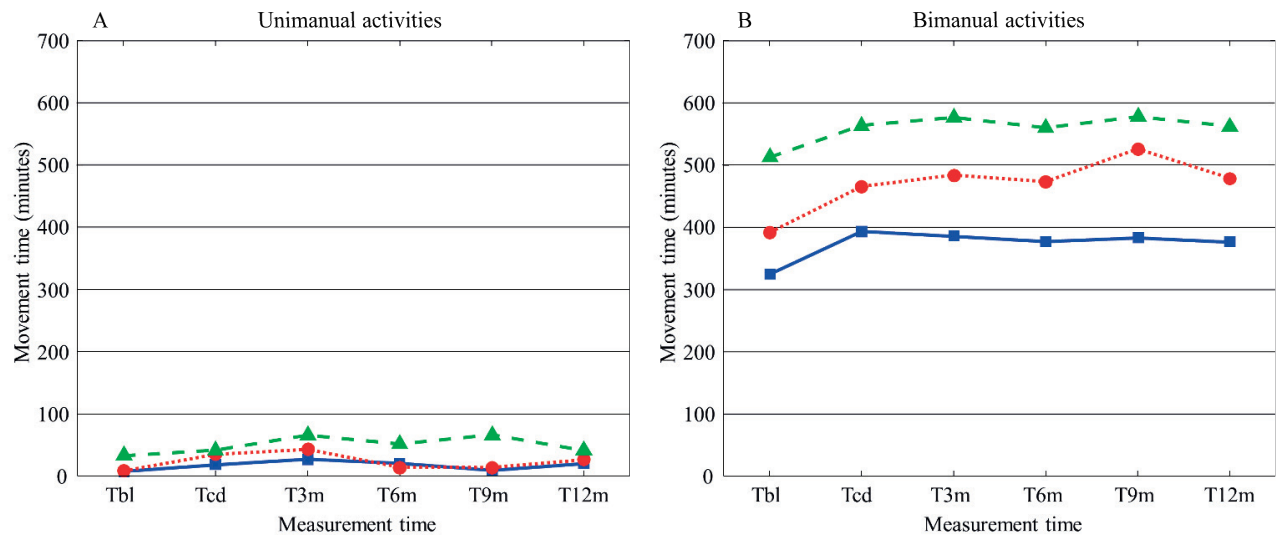

Figure 5A. Mean values for Duration-of-arm-hand-use on the affected side during unimanual activities.

Figure 5B. Mean values for Duration-of-arm-hand-use on the affected side during bimanual activities for subgroups 1 , 2 and 3. $T=$ time; $b l=$ baseline; $c d=$ clinical discharge; $m=$ month. Solid line = subgroup $1 ;$ Dotted line = subgroup 2; Dashed line = subgroup 3 . 


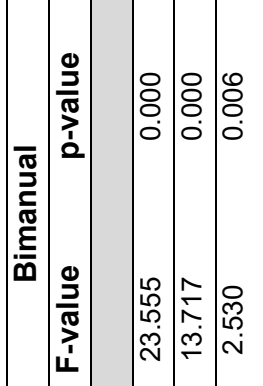

:

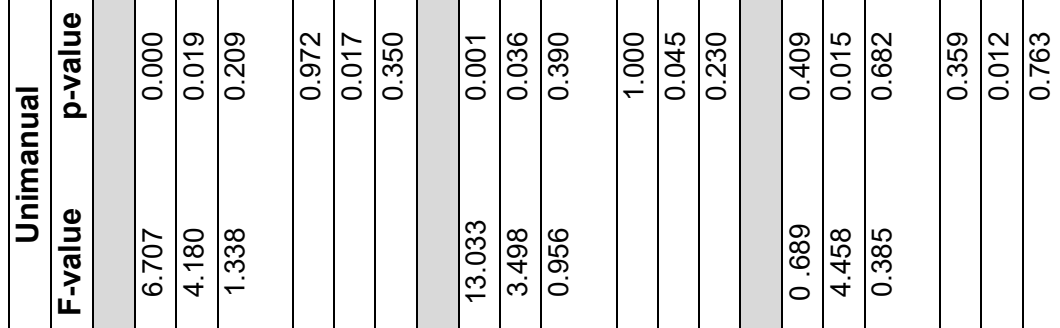

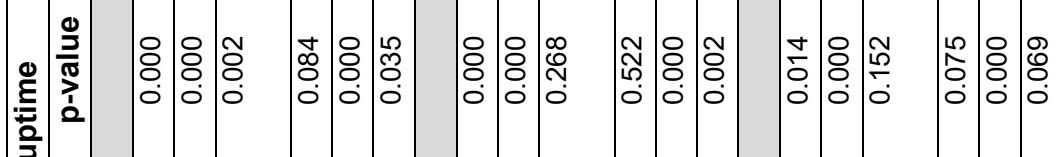

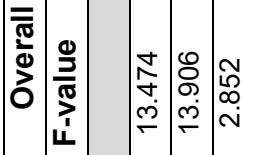

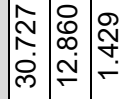

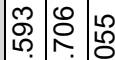

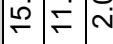

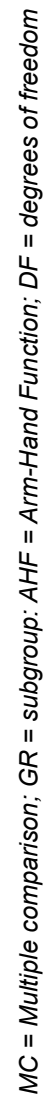


Although between $T_{B L}$ and $T_{C D}$, and also from $T_{B L}$ across to $T_{12 m}$ main significant effects for 'Time' and 'AHF status' were found regarding Duration-of-arm-hand-use on the affected side during unimanual activities, differences were rather small. Most distinct differences between subgroups at all points in time were found between subgroup 1 and 3 .

Duration-of-arm-hand-use on the affected side during bimanual activities

Mean values for Duration-of-arm-hand-use on the affected side during bimanual activities for subgroups 1, 2 and 3 are also displayed in Figure 5B. The corresponding statistics are presented in Table 3.

Significant main effects for 'Time' and for 'AHF status' regarding Duration-of-arm-handuse on the affected side during bimanual activities were found for the phase between $T_{B L}$ and $T_{C D}$ as well as for the phase between $T_{B L}$ and $T_{12 m}$. Subgroup differences were most prominent between subgroup 1 and 3 . 


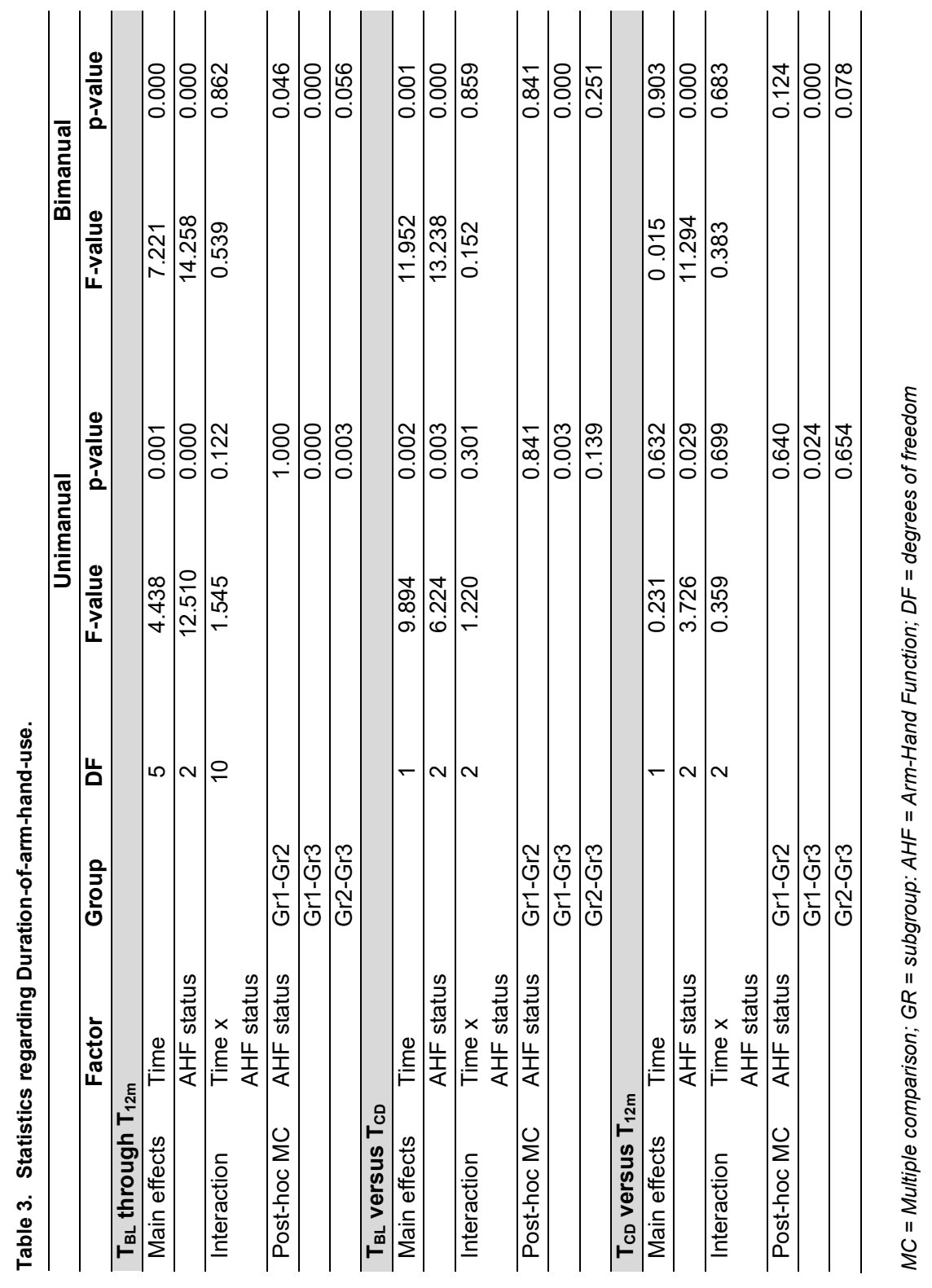


Mean ratios regarding Intensity of arm-hand use between the affected and the nonaffected arm-hand during uptime

Mean ratios regarding Intensity of arm-hand use between the affected and the nonaffected arm-hand during uptime for subgroups 1, 2 and 3 are displayed in Figure 6A. The corresponding statistics are presented in Table 4.

Mean ratios regarding Intensity of arm-hand use between affected and non-affected side
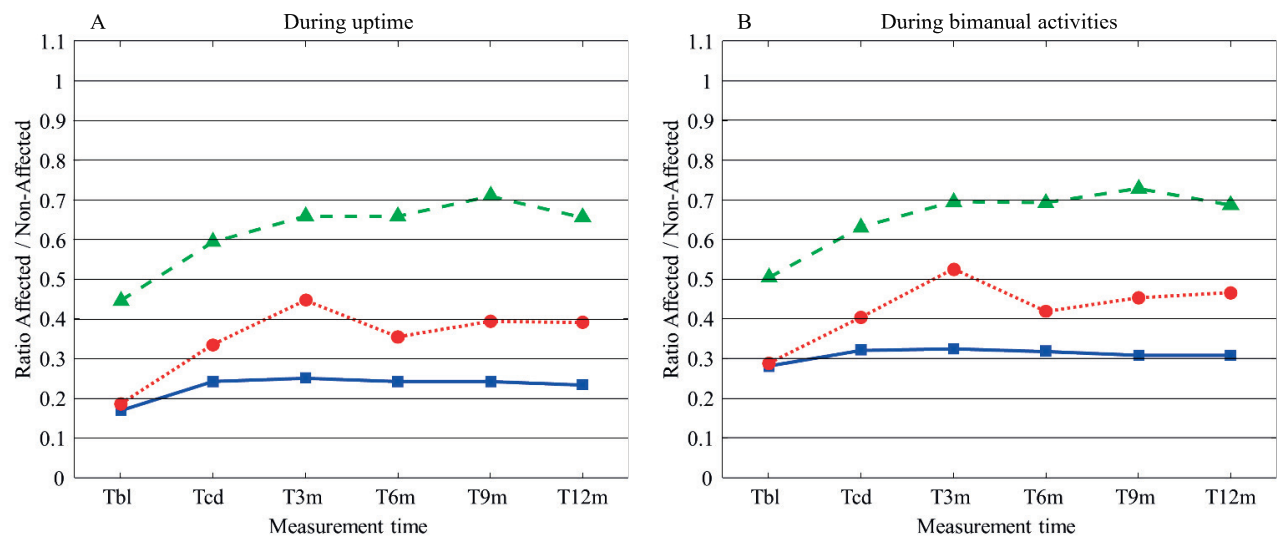

Figure 6A. Mean ratios regarding Intensity of arm-hand use between the affected and the non-affected arm-hand during uptime for subgroups 1, 2 and 3.

Figure 6B. Mean ratios regarding Intensity of arm-hand use between the affected and the non-affected arm-hand during bimanual activities for subgroups 1, 2 and 3. $T=$ time; $b l=$ baseline; $c d=$ clinical discharge; $m=$ month; $A=$ affected side; $N A=$ non-affected side. Solid line = subgroup 1; Dotted line = subgroup 2; Dashed line = subgroup 3.

During uptime the ratio of Intensity-of-arm-hand-use between the affected and nonaffected side increased in all subgroups across the clinical and post-clinical period, with major gains observed between $\mathrm{T}_{\mathrm{BL}}$ and $\mathrm{T}_{\mathrm{CD}}$. Also a main effect for 'AHF status', but no 'Time' x 'AHF status' interaction related to Intensity-of-arm-hand-use between the affected and non-affected side during uptime was found throughout the rehabilitation phase and post-clinical phase. 
Mean ratios regarding Intensity of arm-hand use between the affected and the nonaffected arm-hand during bimanual activities

Mean ratios regarding Intensity of arm-hand use between the affected and the nonaffected arm-hand during bimanual activities for subgroups 1,2 and 3 are displayed in Figure 6B. The corresponding statistics are presented in Table 4.

As to the ratio of Intensity-of-arm-hand-use between the affected and non-affected side during bimanual activities, similar results were observed as described by the uptimerelated ratios above.

\section{Discussion}

The aim of the present study was to assess a) possible improvement or deterioration in actual arm-hand use of sub-acute stroke patients during and after clinical rehabilitation involving a well-defined therapy approach, and b) to what extent actual arm-hand use differs between three subgroups of stroke patients, i.e. patients with either a severely, moderately or mildly affected arm-hand, within this time period. The majority of the study population significantly improved as to Intensity-of-arm-and-handuse during waking hours (or so-called 'uptime'). However, when differentiating between unimanual and bimanual task conditions, during the rehabilitation period, i.e. between baseline and clinical discharge, Intensity-of-arm-and-hand-use on the affected side during unimanual activities sharply dropped. In contrast, in the exact same period of time bimanual activities the Intensity-of-arm-and-hand-use on the affected side increased. This indicates that the patients learn to co-use their affected arm-hand more during bimanual skill performance. 


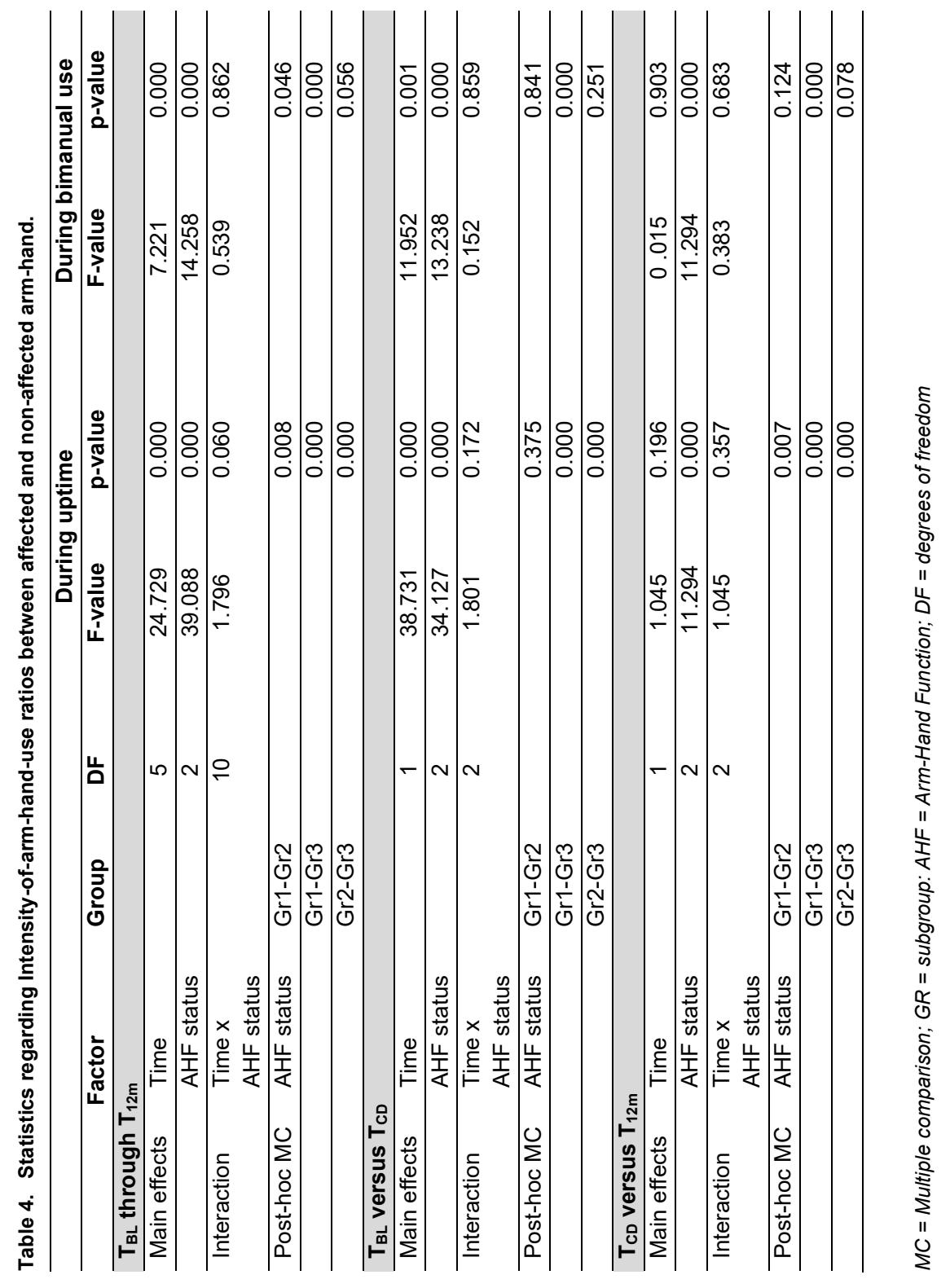


Duration-of-arm-hand-use on the affected side during bilateral activities improved, especially during the rehabilitation phase, and remained at this higher level during the post-clinical phase. In contrast, Duration-of-arm-hand-use on the affected side during unilateral activities remained at nearly identical levels throughout the rehabilitation and post-rehabilitation phase. These results on actual arm-hand use are in line with improvements observed on both function level and capacity level in the same group as presented by Franck et al. [15].

\section{Use of the affected hand unimanually}

\section{Intensity-of-arm-hand-use}

In moderately or severely affected patients, the intensity of unimanual arm-hand use on the affected side sharply dropped during the rehabilitation phase, as was also reported by Rand et al. [18]. This phenomenon was less in the mildly affected group. This may have been due to the fact that, during arm-hand treatment, especially the moderately impaired patients are vigorously encouraged to use their affected armhand in bimanual activities during their training period in program 2. The mildly impaired patients, are, due to their initial state of impairment, already inclined to work bimanually, using their affected hand less unimanually. A small scale study by Urbin et al. [30] reported similar results in a group of patients in sub-acute phase comparable with the moderately and mildly affected group as presented in this study. During the post-clinical phase, the intensity of unilateral arm-hand use on the affected side remains low. One year after discharge, patients with a moderately affected arm-hand function achieved and maintained a certain level of intensity of use of the affected hand in unimanual conditions. In contrast, De Niet et al. and Michielsen et al. [54, 55], found that chronic stroke patients hardly use their affected arm-hand unimanually.

Despite a difference in absolute values regarding the Intensity-of-arm-hand-use, relative progressions gained in the moderately affected group in the post-rehabilitation phase did not differ significantly from those in mildly impaired patients. This is interesting, because, in contrast to persons with a mildly impaired arm-hand, patients with a moderately impaired arm-hand first had to regain a substantial level of dexterity as a prerequisite before being able to actually use their affected arm-hand in daily task performance. 


\section{Duration-of-arm-hand-use}

Whereas Intensity-of-arm-hand-use during unimanual activities showed a significant decline during the rehabilitation phase, especially in persons with a mildly and moderately affected hand, Duration-of-arm-hand-use of the affected hand improved in both groups during the exact same period of time. Results achieved during the rehabilitation phase were maintained across the post-rehabilitation phase, which is interesting in particular regarding the moderately impaired group, in which a substantial part of the patients started with no dexterity at all [15]. These results may probably be a consequence of the patient's learning process to reintegrate the affected hand during skill performance tasks [56]. From the perspective of a patient with a moderately affected hand, gaining confidence in task performance is a powerful issue that may lead to a more positive belief in his/her own capabilities to achieve levels of arm-hand performance previously set out for during goal setting, and may even lead to inter-task transfer of learning towards other, untrained functional tasks [57].

\section{Use of the affected arm-hand bimanually}

\section{Intensity-of-arm-hand-use}

Regarding bimanual activities, patients with a moderately or mildly impaired hand learned to use their affected hand more frequently and more intensively during the rehabilitation phase and maintained that level till one year after discharge. Similar results were found by Michielsen et al. [55].

Despite an absolute difference in the values regarding Intensity-of-arm-hand-use as measured between the mildly and moderately affected group, the latter group showed a similar pattern of progression in bimanual arm-hand use, both during and after the rehabilitation phase.

To improve and to maintain a certain level of Intensity-of-arm-hand-use in the moderately affected hand used during bimanual task performance is challenging for these patients. They suffer from no or almost no dexterity at the start of the arm-hand rehabilitation process [15]. For these patients it takes courage to use their affected hand in bimanual activities and become satisfied about how activities are performed and accomplished [58]. 
The majority of patients with a severely impaired hand remained unable to use their hand during bimanual performance of tasks in this same period. Progressions observed regarding Intensity-of-arm-hand-use may be explained by: 1) an increase of voluntary movements in flexion synergy in the proximal and/or distal part of the arm; 2) associated movements while moving or performing activities with the non-affected hand or during walking; and 3) performing bimanual exercises as learned to maintain the severely affected arm-hand supple and pain free. However, in contrast to the majority of patients mentioned above who followed program 1 , six patients in the severely affected group did show considerable (early) arm-hand improvement. This may be associated with spontaneous recovery. This improvement made them eligible for training in CARAS program 2, whereas all data from the severely impaired patient subgroup were analyzed according to the intention-to-treat principle. A per-protocol analysis of the data (not reported here) leaving out these six patients, showed that in the severely affected group neither Intensity-of-arm-hand-use nor Duration-of-armhand-use on the affected side improved significantly across time.

The differences in Intensity-of-arm-hand-use, as observed between the three groups, may be interpreted as follows: 1) In contrast to severely and moderately affected patients, mildly affected patients display voluntary wrist and finger movements at the initial phase of the arm-hand rehabilitation program, associated with a certain degree of cortico-spinal tract integrity [59,60]. Due to this substantial spontaneous recovery episode, mildly impaired patients become enabled to integrate their affected hand relative more quickly in (bimanual) tasks compared to moderately/severely affected patients. 2) The course of arm-hand rehabilitation of the mildly affected group is less interrupted by problems more commonly seen in the other two groups, like, for instance, presence of cognitive deficits, which could influence motor (re)learning negatively [61]; the appearance of weakness i.e. loss of strength and change in muscle condition [62]; the change in the paretic shoulder's physical and kinematic properties which results in a less than optimal scapular joint alignment [63]; or swelling or edema of the post-stroke hand [64]. Patients with a moderately affected hand, admitted to CARAS, program 2, went through a considerable development process from not being able to use the affected hand during daily activities at the start of the arm-hand training 
[15], towards displaying dexterity and a concomitant higher level of Intensity-of-armhand-use during rehabilitation and in daily life performance after rehabilitation.

Regarding the moderately and mildly affected group, Uswatte et al. Taub et al. and Liao et al. reported similar findings i.e. a significant increase of Intensity-of-arm-handuse in sub-acute and chronic stroke patients with a moderately or mildly affected armhand who participated in a constrained-induced therapy program [44, 65]. Liao et al. combined functional training with robot practice in mildly impaired chronic stroke patients [66]. However, in contrast to the present study, these studies included relative small study populations with strict inclusion criteria, thus reducing generalizability.

No significant difference were reported by Doman et al. and Rand et al. regarding Intensity-of arm-hand-use in sub-acute stroke patients with a moderately to mildly affected hand, who received arm-hand training [18, 43]. Waddel et al. reported no significant differences in Intensity-of-arm-hand-use in moderately and mildly impaired chronic ( $\geq 6$ months) stroke patients after the rehabilitation phase who participated in a high repetitive, task-specific arm-hand regime [67].

\section{Duration-of-arm-hand-use}

In moderately and mildly impaired patients improvement in the Duration-of-arm-handuse of the affected hand during bimanual task performance was observed. However, relative to the non-affected arm-hand, the affected arm-hand continued to play a limited role, which is in line with the studies of Bailey and Michielsen [29, 55].

In mildly impaired patients in the post-rehabilitation period, the non-affected hand is used about one and a half times more than the affected hand. In this same period, patients with a moderately affected hand used their non-affected hand about two and a half times more than their affected hand. Thrane et al. (2011) found a difference in this ratio of up to two and a half times in a group of sub-acute stroke patients with a mildly impaired arm-hand who are comparable with the mildly impaired group as presented in the present study [68]. In contrast, healthy older adults of approximately the same age as the study participants display a more equal Intensity-of-use between both hands [55, 69-71]. Regarding the mildly impaired group the reduction in bilateral arm-hand activity might be due to (a combination of) a lack of motor capability, patient 
motivation, a shift in routine performance tasks, or a direct result of so-called learned non-use [20, 72].

The moderately affected group as presented in the present study demonstrated far less motor capabilities at the initial start of the rehabilitation phase compared to the mildly affected group [15], which may explain the lower level of bimanual performance in the moderately affected group. Despite this low level, data obtained from the present study suggests that these patients re-learn to use their the affected arm-hand during (bi)manual task performance during their rehabilitation period. Within this early, subacute phase these patients become enabled to perform a vast number of bimanual tasks in their daily life situation, as learned within program 2 of CARAS. These findings differ from the study of Waddel et al. [67], who reported no improvements in arm hand use in daily performance, after completing an eight-week intensive task-specific armhand program. However, their study population consisted of chronic ( $\geq 6$ months postinjury) stroke patients with, on average, a level of arm-hand capacity similar to our moderately affected group measured at clinical discharge. In the post-stroke phase, the moderately affected hand is used in bimanual activities, but still proportionally less than the non-affected hand. The ratio between the affected and non-affected arm-hand remained more or less unchanged during the post-clinical phase. This suggests that patients may have become accustomed to use their affected hand regularly in skill performance in daily situations, a phenomenon also described by other authors [18, $55,67]$.

Clinically speaking, sub-acute stroke patients who followed an arm-hand training featuring CARAS, program 2 and program 3, improved on Intensity-of-use and Duration-of-use of their moderately or mildly affected arm-hand.

\section{Considerations}

This study is not without limitations. First, the inability to differentiate between signals stemming from task-specific (e.g. reaching or grasping) or non-task-specific arm-hand movement may limit the interpretation of results regarding any qualitative aspect of arm-hand motor behavior [30]. Wrist accelerometry is a valid and reliable 
measurement method [20, 31]. However, it measures 'use' and not 'purposeful use'[25], and does not provide information about movement quality or specific activities that were performed during the wearing period. Therefore, data on actual arm-hand use provided in this study have to be interpreted with some care when translating these results towards real arm-hand skill performance. Techniques to identify (the quality of) specific among multiple activities using body worn sensors are upcoming and are promising tools to further improve actual arm-hand skill performance measurement [73].

Secondly, data on actual arm-hand use, based on accelerometry, depend on the way how arm-hand use has been defined, and on how data are collected and processed [20]. The term 'counts', as a measure of 'amount of use' [23, 74], is often used. However, the calculation of 'counts' is not always the same across studies. Therefore, in order to be able to compare our results with other studies, in the present study it is explicitly formulated how the 'amount of use' metric was calculated, based on the raw accelerometer data of the Actiwatches used. This makes careful comparisons with other studies regarding the use and interpretation of accelerometry data possible.

Thirdly, in the present study an intention-to-treat analysis has been performed, which may have resulted in substantial larger within-group variances in the three subgroups. In the severely affected group some patients showed substantial large progressions regarding Intensity-of-arm-hand-use during the clinical rehabilitation phase, and were transferred from CARAS program 1 to CARAS program 2 during the rehabilitation phase. These improvements in a subpopulation in the severely affected subgroup could explain the statistically significant differences being found. A per-protocol analysis of the data (data not reported in this paper) revealed that in the remainder of participants in subgroup 1 neither a statistical nor a clinically relevant improvement in actual arm-hand use was found.

Fourthly, contrary to participants from subgroup 1 and 3, patients with a moderately affected arm-hand received a second six weeks period of training in order to experience functionally meaningful progressions in the use of their affected hand and 
the possibility to use their affected arm-hand more purposefully in bimanual skill performance.

Fifthly, in the present study a distinctly low proportion of female participants participated (see Table 1), especially in subgroup 2 and 3. This may have influenced our results. In general, most instrumental activity of daily living (IADL) tasks which require arm-hand usage are traditionally done by women. Though physically capable to perform IADL tasks, men often rely on their spouses performing IADL tasks like cooking and laundry [75]. However, despite this unequal representation of gender across both groups, group results indicate progression in both 'intensity-of-arm-handuse' and 'duration-of-arm-hand-use'. Subgroup 1 contained proportionally more female participants compared to subgroup 2 and subgroup 3. Therefore, gender differences most likely had even less influence on study results regarding the latter group.

Sixthly, the average post-stroke time at point of admission to the study of patients of subgroup 1 differed from the average post-stroke time in patients admitted to subgroup 2 and 3 (see Table 1). This is a phenomenon typically seen in clinical situations in the sub-acute phase after stroke. In contrast to moderately and mildly impaired patients, who were trained to increase intensity and duration of use of their affected hand, patients of subgroup 1 were encouraged to keep their severely affected arm in good condition instead of being stimulated, to no avail, to regain arm-hand use [14]. This has led to patients from different groups to improve at a different rate, leading to between-group differences. Another source of between-group differences is, of course, the aforementioned difference in stroke severity at entry into the study.

\section{Acknowledgements}

We are grateful to all patients who participated in this study and to all therapists who contributed to this study. 


\section{References}

1. Mayo NE, Wood-Dauphinee S, Cote R, Durcan L, Carlton J. Activity, participation, and quality of life 6 months poststroke. Archives of physical medicine and rehabilitation. 2002;83(8):1035-42.

2. Guidetti S, Ytterberg C, Ekstam L, Johansson U, Eriksson G. Changes in the impact of stroke between 3 and 12 months poststroke, assessed with the stroke impact scale. J Rehabil Med. 2014;46:963-8.

3. Gebruers N, Vanroy C, Truijen S, Engelborghs S, De Deyn P. Monitoring of physical activity after stroke: a systematic review of accelerometry-based measures. Archives of physical medicine and rehabilitation. 2010;91:288-97.

4. Sveen U, Bautz-Holter E, Sodring K, Wyller T, Laake K. Association between impairments, self-care ability and social activities 1 year after stroke. Disabililty and Rehabilitation. 1999;21(8):372 - 7.

5. Kwakkel G, Kollen B, Twisk J. Impact of time on improvement of outcome after stroke. Stroke; a journal of cerebral circulation. 2006;37(9):2348-53. PubMed PMID: 16931787.

6. Pollock A, Farmer SE, Brady MC, Langhorne P, Mead GE, Mehrholz J, et al. Interventions for improving upper limb function after stroke. The Cochrane database of systematic reviews. 2014;11:CD010820. Epub 2014/11/12. doi: 10.1002/14651858.CD010820.pub2. PubMed PMID: 25387001.

7. Törnbom K, Persson H, Lundälv J, Sunnerhagen KS. Self-Assessed Physical, Cognitive, and Emotional Impact of Stroke at 1 Month: The Importance of Stroke Severity and Participation. Journal of Stroke and Cerebrovascular Diseases. 2016;26(1):57 - 63.

8. Wolf $\mathrm{T}$, Koster J. Perceived recovery as a predictor of physical activity participation after mild stroke. Disability and rehabilitation. 2013;35:1143 - 8.

9. Franceschini M, La Porta F, Agosti M, Massucci M. Is health-related-quality of life of stroke patients influenced by neurological impairments at one year after stroke? European journal of physical and rehabilitation medicine. 2010;46(3):389-99. Epub 2010/10/12. PubMed PMID: 20927005.

10. Wolfe CD. The impact of stroke. Br Med Bull. 2000;56(2):275-86. PubMed PMID: 11092079.

11. Nichols-Larsen DS, Clark PC, Zeringue A, Greenspan A, Blanton S. Factors influencing stroke survivors' quality of life during subacute recovery. Stroke; a journal of cerebral circulation. 2005;36(7):1480-4. PubMed PMID: 15947263.

12. Wyller T, Sveen U, Sødring K, Pettersen AM, Bautz-Holter, E. Subjective well-being one year after stroke. Clinical rehabilitation. 1997;11(2):139 - 45. 
13. Veerbeek JM, van Wegen E, van Peppen R, van der Wees PJ, Hendriks E, Rietberg M, et al. What is the evidence for physical therapy poststroke? A systematic review and metaanalysis. PloS one. 2014;9(2):e87987. Epub 2014/02/08. doi: 10.1371/journal. pone.0087987. PubMed PMID: 24505342; PubMed Central PMCID: PMCPMC3913786.

14. Franck JA, Halfens JAM, Smeets RJEM, Seelen HAM. Concise Arm and hand Rehabilitation Approach in Stroke (CARAS): A practical and evidence-based framework for clinical rehabilitation management. The Open Journal of Occupational Therapy. 2015;3(4):Article 10. doi: 10.15453/2168-6408.1164.

15. Franck JA, Smeets RJEM, Seelen HAM. Changes in arm-hand function and arm-hand skill performance in patients after stroke during and after rehabilitation. PloS one. 2017;12(6):1 - 18.

16. WHO. International classification of functioning, disability and health. 2001.

17. Lemmens RJ, Timmermans AA, Janssen-Potten YJ, Smeets RJ, Seelen HA. Valid and reliable instruments for arm-hand assessment at ICF activity level in persons with hemiplegia: a systematic review. BMC Neurol. 2012;12:21. Epub 2012/04/14. doi: 10.1186/1471-2377-12-21. PubMed PMID: 22498041; PubMed Central PMCID: PMCPMC3352056.

18. Rand D, Eng JJ. Disparity between functional recovery and daily use of the upper and lower extremities during subacute stroke rehabilitation. Neurorehabil Neural Repair. 2012;26:76-84.

19. Rand D, Eng JJ. Predicting Daily Use of the Affected Upper Extremity 1 Year after Stroke. Journal of Stroke and Cerebrovascular Diseases. 2015;24(2):274 - 83.

20. Noorkoiv M, Rodgers H, Price C. Accelerometer measurement of upper extremity movement after stroke: a systematic review of clinical studies. Journal of neuroengineering and rehabilitation. 2014;11(144):1 - 11.

21. Mallinson T, Hammel J. Measurement of Participation: Intersecting Person, Task, and Environment. Archives of physical medicine and rehabilitation. 2010;91:29 - 33.

22. Raugh A, Cieza A, Stucki G. How to apply the International Classification of Functioning, Disability and Health (ICF) for rehabilitation management in clinical practice. European journal of physical and rehabilitation medicine. 2008;44:329 - 42.

23. Lang C, Marghuretta D, Bailey R, Schaefer S, Birkenmeier R. Assessment of upper extremity impairment, function, and activity following stroke: Foundations for clinical decision making J Hand Ther. 2013;26:104 - 15.

24. Niet M, Bussmann JB, Ribbers GM, Stam H. The Stroke Upper-Limb Activity Monitor: Its Sensitivity to Measure Hemiplegic Upper-Limb Activity During Daily Life.

Archives of physical medicine and rehabilitation. 2007;88:1121 - 6 . 
25. Uswatte G, Foo WL, Olmstead H, Lopez K, Holand A, Simms LB. Ambulatory monitoring of arm movement using accelerometry: an objective measure of upper-extremity rehabilitation in persons with chronic stroke. Archives of physical medicine and rehabilitation. 2005;86(7):1498-501. PubMed PMID: 16003690.

26. Vega-Gonzalez A, Granat MH. Continuous monitoring of upper-limb activity in a free-living environment. Archives of physical medicine and rehabilitation. 2005;86(3):541 - 8.

27. Connell L, McMahon N, Simpson L. Investigating measures of intensity during a structured upper limb exercise programme in stroke rehabilitation:

an exploratory study. Archives of physical medical rehabilitation. 2014;95.

28. Van der Pas SC, Verbunt JA, Breukelaar DE, van Woerden R, Seelen HA. Assessment of arm activity using triaxial accelerometry in patients with a stroke. Archives of physical medicine and rehabilitation. 2011;92.

29. Bailey RR, Klaesner JW, Lang CE. Quantifying real-world upper limb activity in nondisabled adults and adults with chronic stroke. Neurorehabilitation and neural repair. 2015;29(10):969-78.

30. Urbin M, Waddel K, Lang CE. Acceleration metrics are responsive to change in upper extremity function of stroke survivors. Archives of physical medicine and rehabilitation. 2015;96(5):854 - 61 .

31. Lee J, Kwon S, Kim W, Hahn S, Park J, Paik N. Feasibility, reliability, and validity of using accelerometers to measure physical activities of patients with stroke during inpatient rehabilitation. PloS one. 2018;13(12).

32. Hayward KS, Eng J, Boyd L, Lakhani B, Bernhardt J, Lang C. Exploring the Role of Accelerometers in the Measurement of Real World Upper-Limb Use After Stroke. Brain Impairment. 2016:1-18.

33. Block V, Pitsch E, Tahir P, Cree B, Allen D, Gelfland J. Remote Physical Activity Monitoring in Neurological Disease: A Systematic Review. PloS one. 2016.

34. Lemmens JM, Timmermans AA, Janssen-Potten YJM, Pulles SA, Geers RP, Bakx WG, et al. Accelerometry measuring the outcome of robot-supported upper limb training in chronic stroke: a randomized controlled trail. PloS one. 2014. doi: 10.1371/journal.pone.0096414.

35. Narai E, Hagino H, Komatsu T, Togo F. Accelerometer-Based Monitoring of Upper Limb Movement in Older adults With Acute and Subacute Stroke. J Geriatr Phys Ther. 2016;39:171-7.

36. Van der Pas S, Verbunt AJ, Breukelaar D, van Woerden R, Seelen H. Assessment of Arm Activity Using Triaxial Accelerometry in Patients With a Stroke. Archives of physical medicine and rehabilitation. 2011;92:1437 - 42. 
37. Uswatte G, Giuliani C, Winstein C, Zeringue A, Hobbs L, Wolf SL. Validity of accelerometry for monitoring real-world arm activity in patients with subacute stroke: evidence from the extremity constraint-induced therapy evaluation trial. Archives physical medical rehabilitation. 2006;87:1340 - 5.

38. Gebruers N, Truijen S, Engelborghs S, Nagels G, Brouns R. Actigraphic Measurement of Motor Deficits in Acute Ischemic Stroke. Cerebrovascular Disease. 2008;26:533 - 40.

39. Withford MR, M. Effects of in home high dose accelerometer-based feedback on perceived and actual use in participants chronic post-stroke. Physiotherapy Theory and Practice. 2018:1-12.

40. Liao WW, Wu CY, Hsieh YW, Lin KC, Chang WY. Effects of robot-assisted upper limb rehabilitation on daily function and real-world arm activity in patients with chronic stroke: a randomized controlled trial. Clinical rehabilitation. 2012;26(2):111-20.

41. Lang CE, MacDonald JR, Gnip C. Counting repetitions: an observational study of outpatient therapy for people with hemiparesis post-stroke. J Neurol Phys Ther. 2007;31(1):3-10. PubMed PMID: 17419883.

42. Vanroy $\mathrm{C}$, Vanlandewijck $\mathrm{Y}$, Cras $\mathrm{P}$, Feys $\mathrm{H}$, Truijen $\mathrm{S}$, Michielsen $\mathrm{M}$, et al. Is a Coded Physical Activity Diary Valid for Assessing Physical Activity Level and Energy Expenditure in Stroke Patients? PloS one. 9(6):1 - 8.

43. Doman C, Waddell K, Bailey R, Moore J, Lang C. Changes in Upper-Extremity Functional Capacity andDaily Performance During Outpatient Occupational Therapy for People With Stroke. The American Journal of Occupational Therapy. 2016;70.

44. Taub EU, Uswatte G. Constraint-Induced Movement Therapy: A Family of Neurorehabilitation Treatments that Harnesses the Plasticity of the Central Nervous System. Neurol Rehabil 2013;19(3):161-75.

45. Uswatte G, Taub E, Morris D, Vignolo M, McCulloch K. Reliability and validity of the upperextremity Motor Activity Log-14 for measuring real-world arm use. Stroke; a journal of cerebral circulation. 2005;36(11):2493-6. PubMed PMID: 16224078.

46. Timmermans AA, Lemmens RJ, Monfrance M, Geers RP, Bakx WM, Smeets RJ, et al. Effects of task-oriented robot training on arm function, activity, and quality of life in chronic stroke patients: a randomized controlled trail. Journal of neuroengineering and rehabilitation. 2014;31:11-45.

47. Uswatte G, Giuliani C, Winstein C, Zeringue A, Hobbs L, Wolf SL. Validity of accelerometry for monitoring real-world arm activity in patients with subacute stroke: evidence from the extremity constraint-induced therapy evaluation trial. Archives of physical medicine and rehabilitation. 2006;87(10):1340-5. PubMed PMID: 17023243.

48. Prabhakaran S, Zarahn E, Riley C, Speizer A, Chong JY, Lazar RM, et al. Inter-individual variability in the capacity for motor recovery after ischemic stroke. Neurorehabilitation and 
neural repair. 2008;22(1):64-71. Epub 2007/08/10. doi: 10.1177/1545968307305302. PubMed PMID: 17687024.

49. Winters C, van Wegen EE, Daffertshofer A, Kwakkel G. Generalizability of the Proportional Recovery Model for the Upper Extremity After an Ischemic Stroke. Neurorehabilitation and neural repair. 2015;29(7):614-22. Epub 2014/12/17. doi: 10.1177/1545968314562115. PubMed PMID: 25505223.

50. Kruitwagen-van Reenen ET, Post MW, Mulder-Bouwens K, Visser-Meily JM. A simple bedside test for upper extremity impairment after stroke: validation of the Utrecht Arm/Hand Test. Disability and rehabilitation. 2009;31(16):1338-43.

51. Cambridge_Neurology_Itd. The activitywatch guide to getting started. Cambridgde: CamNtech_Ltd. 2008.

52. Twisk JWR. Applied longitudinal data analysis for epidemiology. A practical guide. . Cambridge: Cambridge University Press; 2003.

53. Shapiro SS, Wilk MB. an analysis of variance test for normality (complete samples). Biometrica. 1965;52(3-4):591-611.

54. De Niet MB, JB. Ribbers, GM. Stam, H. The stroke upper-limb activity monitor: its sensitivity to measure hemiplegic upper-limb activity during daily life. Archives of Physical Medical Rehabilitation. 2007;88:1121 - 6.

55. Michielsen M, Selles R, Stam H, Ribbers G, Bussman J. quantifying nonuse in chronic stroke patients: a study into paretic, nonparetic and bimanual upper limb use in daily life. Archives of physical medicine and rehabilitation. 2012;93:1975-81.

56. Buma F, Kwakkel G, Ramsey N. Understanding upper limb recovery after stroke. Restorative Neurology and Neuroscience. 2013;31:707 - 22.

57. Schaefer S, Patterson C, Lang C. Transfer of Training Between Distinct Motor Tasks After Stroke: Implications for Task- Specific Approaches to Upper-Extremity Neurorehabilitation.Neurorehabilitation and neural repair. 2013;27(7):602-12.

58. Barker RN, Brauer SG. Upper limb recovery after stroke: the stroke survivors' perspective. Disability and rehabilitation. 2005;27(20):1213-23. Epub 2005/11/22. doi: 10.1080/09638280500075717. PubMed PMID: 16298923.

59. Jang SH, Ahn SH, Yang DS, Lee DK, Kim DK, Son SM. Cortical reorganization of hand motor function to primary sensory cortex in hemiparetic patients with a primary motor cortex infarct. Archives of physical medicine and rehabilitation. 2005;86(8):1706-8. PubMed PMID: 16084830.

60. Santello M. Synergistic Control of Hand muscles through common neural input. In: balasubramanian S, Santos V, editors. the human hand as an inspiration for robot hand development. 1. Switserland: Springer 2014. 
61. Mullick AA, Subramanian SK, Levin MF. Emerging evidence of the association between cognitive deficits and arm motor recovery after stroke: A meta-analysis. restorative Neurology and Neuroscience. 2015;33(3):389 - 403.

62. Canning CG, Ada L, Adams R, O'Dwyer NJ. loss of strength contributes more to physical disability after stroke than loss of dexterity. Clinical rehabilitation. 2004;18(3):300 - 8.

63. Hardwick D, Lang C. Scapular and humeral movement patterns of people with stroke during range of motion exercises. Journal of Neurological Physical Therapy. 2011;35(1):18 - 25.

64. Boomkamp-Koppen HG, Visser-Meily JM, Post MW, Prevo AJ. poststroke hand swelling and oedema: prevalence and relationship with impairment and disability. Clinical rehabilitation. 2005;19(5):552-9.

65. Uswatte G, Taub E, Morris D, Barman J, Crago J. Contribution of the shaping and restraint components of Constraint-Induced Movement therapy to treatment outcome. NeuroRehabilitation. 2006;21(2):147-56. PubMed PMID: 16917161.

66. Liao W, Wu C, Hsieh Y, Lin K, Chang W. Effects of robot-assisted upper limb rehabilitation on daily function and real-world arm activity in patients with chronic stroke: a randomized controlled trial. Clinical rehabilitation. 2011;26:111-20.

67. Waddel K, Strube M, Bailey R, Klaesner J, Birkenmeier R, Dromerick A, et al. Does taskspecific training improve upper limb performance in daily life post-stroke? Neurorehabilitation and neural repair. 2017;31(3):290 - 300.

68. Thrane G, Emaus N, Askim T, Audney A. Arm use in patients with subacute stroke monitored by accelerometry: association with motor impairment and influence on selfdependence. J Rehabil Med. 2011;43:299-304.

69. Lang CE, Wagner JM, Edwards DF, Dromerick AW. Upper extremity use in people with hemiparesis in the first few weeks after stroke. Journal of Neurologic Physical Therapy. 2007;31:56-63.

70. Kilbreath S, Heard R. Frequency of hand use in healthy older persons. Australian Journal of Physiotherapy. 2005;51(119-122).

71. Bailey RR, Klaesner JW, Lang CE. Quantifying real-world upper-limb activity in nondisabled adults and adults with chronic stroke. Neurorehabilitation and neural repair. 2015;29:969-78.

72. Taub E, Uswatte G, Mark VW, Morris DM. The learned nonuse phenomenon: implications for rehabilitation. Eura Medicophys. 2006;42(3):241-56. PubMed PMID: 17039223.

73. Lemmens JM, Timmermans AA, Janssen-Potten YJM, Pulles S, Bakx WGM, Smeets RJEM, et al. recognizing complex upper extremity activities using body worn sensors. PloS one. $2015 ; 10(3)$. 
74. Chen Y, Basset D. The Technology of Accelerometry-Based Activity Monitors: Current and Future. Medicine \& Science in Sports \& Exercise. 2005;37(11):490-500.

75. Rand D, Janice JE. Arm-Hand Usage in Healthy Older Adults. Am J Occup Ther. 2010;64(6):877-85. 


\section{Chapter}

\section{Added-value of early post-stroke spasticity reduction during arm-hand rehabilitation in improving functional arm-hand skill performance: A multiple baseline single case experimental design study}

Johan Anton Franck, Rob Johannes Elise Marie Smeets, Karolien Renders, Henk Alexander Maria Seelen 


\section{Abstract}

Background: Focal spasticity management in patients in the sub-acute phase after a stroke is mainly based on expert opinion. Evidence for the optimal type and intensity of multidisciplinary rehabilitation programs is scarce. This study will investigate the added-value of reduction of early signs of spasticity in the sub-acute phase after stroke on arm-hand rehabilitation outcome involving a well-described rehabilitation intervention. Reduction of early signs of spasticity will be done using abobotulinum toxin-A.

Methods / design: This study comprises three methodological approaches, i.e.: i) a (multiple baseline) single case experimental design involving 10 individuals; ii) a metaanalysis of the data of all single cases (= single armed group design); iii) nonrandomised double armed group design, i.e. a case-matched control design in which each patient receiving early post-stroke spasticity reduction treatment will be matched (according to arm-hand capacity, spasticity level, age, gender, and Utrecht Arm-hand Test scores) to a case from a prospective cohort study on changes in arm-hand status in 89 stroke patients performed previously.

Improvement of arm-hand skill performance will be gauged using a) the Action Research Arm Test, gauging functional capacity; b) the ABILHAND, gauging perceived level of arm-hand skill proficiency; and c) bilateral arm accelerometry, gauging actual arm-hand skill performance in daily life. Furthermore, arm-hand function will be measured using a) Fugl-Meyer Motor Assessment; b) hand-held dynamometry (grip strength); c) Motricity Index (functional strength); and d) Modified Ashworth Scale (spasticity levels in the upper extremity).

Statistical analyses include permutation tests involving the time series of each subject separately, Kruskal-Wallis tests and Mann-Whitney U-tests.

Discussion: Results of this study will provide evidence on the added-value of reduction of early signs of spasticity in the upper extremity on functional arm-hand skill performance in sub-acute stroke patients with either a severely or moderately affected arm-hand and moderate to severe grades of spasticity. 


\section{Introduction}

In stroke survivors, the presence or absence of voluntary motor activity in the affected arm and hand is the most important predictor of dexterity outcome and the level of performance regarding daily activities. In order to select the potentially most effective treatment, it is advocated to stratify persons with an impaired arm and hand into a limited number of arm-hand function strata [1, 2].

A Concise Arm and hand Rehabilitation Approach in Stroke (CARAS) [3] has been developed to structure and implement the treatment of arm-hand function (AHF) and arm-hand skill performance (AHSP) in stroke survivors. CARAS is based on: A) level of arm-hand impairment, B) detailed training descriptions captured in different training modules, C) principles of self-efficacy [4-6], and D) the swift implementation of innovations. Based on the UAT (Utrecht Arm-hand Test) [7] scores, patients are allocated to one of three training programs, i.e. CARAS program 1, aimed at the severely impaired AHF subgroup (UAT 0-1), CARAS program 2 aimed at the moderately impaired AHF subgroup (UAT 2-3) and CARAS program 3, aimed at the mildly impaired AHF subgroup (UAT 4-7). Program 1 is titled 'taking care and prevention'. It is designed for stroke survivors who are not able to use their affected arm and hand for skill performance in daily life situations (non-functional arm-hand). Program 2 and 3 are high intensity, task-oriented arm-hand performance training programs in which patients learn to integrate their affected arm and hand in daily occupations to optimize their overall functional abilities in daily situations. In this part a distinction is made between persons who have a moderately affected arm and hand, i.e. those who are able to use their affected arm and hand for passive and active stabilization tasks, like fixating bread while making a sandwich; and persons with a mildly affected arm and hand, who are able to use their affected arm and hand instantaneously in daily situations. Currently, CARAS has been implemented in everyday stroke rehabilitation practice in a number of rehabilitation centres throughout the Netherlands.

In order to monitor the development, i.e. either progression or deterioration, of both AHF and AHSP during and after rehabilitation, a single-arm prospective cohort study has been performed, involving 89 patients who participated in CARAS. This study, called AMUSE (Activity Monitoring of Upper extremity use in Stroke patients during and after rEhabilitation (CCMO code: NL35681.068.11)), involved data collection at all 
levels of the ICF (International Classification of Function, disability and health [8]) at the start of rehabilitation (baseline), at clinical discharge, and at 3, 6, 9 and 12 months post discharge, thus providing a time series per patient [9].

In a number of stroke patients with a moderate to severely affected arm and hand, moderate to severe grades of spasticity (Modified Ashworth Scale (MAS) scores $1+$ to 3) may occur already in an early, sub-acute phase post-stroke. This may seriously hinder AHF and AHSP treatment, leading to a slowing down of the patient's functional recovery. The problems caused by spasticity can occur at the level of impairment (e.g. restricted joint range of movement, pain and involuntary movement), and at the level of activity and participation (i.e. inability to perform daily activities, and limitations in taking up societal roles like work, family roles and leisure activities) [10].

There is a myriad of literature on the benefits of reducing spasticity on rehabilitation training effects regarding AHF and AHSP in chronic stroke patients (e.g. [11-18]). However, thus far little evidence is available on the added-value of early spasticity reduction during rehabilitation training on the improvement of AHF and AHSP in subacute stroke patients. In a large study $(n>300)$, Shaw et al. [19] recruited stroke patients in the sub-acute phase, but this study could not demonstrate improved active function. Foley et al. [15] reported small benefits as to improved passive AHF. Baker et al found modest but significant results for active AHF, although evidence quality was reported as low to very low [20]. A number of studies explored the added value of arm - hand rehabilitation directly after the injection of botulinum toxin. Prazeres et al. [21] and Wolf et al. [22] did not find botulinum toxin plus rehabilitation to be superior to placebo plus rehabilitation with respect to AHF. Takekawa et al. [23] and Devier et al. [24] demonstrates improved AHF in stroke patients with a moderately to mildly impaired arm-hand who received botulinum toxin in combination with a tailored armhand rehabilitation program. Reducing the influence of the spastic component demands a holistic multidisciplinary approach to combine spasticity management and rehabilitation to optimise the likelihood of positive treatment effects [12, 25-30]. In most cases, a delay between spasticity reduction and improvement of AHF exists, suggesting that motor relearning continues as muscle tone is returning to baseline. The optimal types (modalities, therapy approaches, settings) and intensities of therapy 
for improving activity (active and passive function) in adults with post-stroke spasticity, in the short and longer term, however, are still unclear [31].

To date, evidence for the optimal type and intensity of multidisciplinary rehabilitation programs following focal spasticity management are based on expert opinion only. The present study will investigate the added-value of reduction of early signs of spasticity in the sub-acute phase after stroke on arm-hand rehabilitation treatment outcome involving a well-described rehabilitation intervention ('treatment-as-usual'), i.e. CARAS. This reduction of early signs of spasticity will be done using abobotulinum toxin-A. It is assumed that reduction of spasticity in the upper extremity at an early, sub-acute, stage after stroke may enable the patient to a) exercise more independently at an earlier stage and b) exercise in a wider variety of therapy conditions also featuring more exercise challenges. This, in turn, may lead to better treatment outcome at the ICF (International Classification of Functioning, activity and participation) [8] function level and activity level.

\section{Aims}

The aim of the present study is to investigate the added-value of reduction of early signs of spasticity in the upper extremity on improving functional arm-hand skill performance in sub-acute stroke patients with either a severely or moderately affected arm-hand (Utrecht Arm-hand Test (UAT) [7] score 1-3) and moderate to severe grades of spasticity, i.e. Modified Ashworth Scale (MAS) [32] score 1+ to 3. Therapy-as-usual, involving a regular, well-documented, concise arm-hand rehabilitation treatment (called CARAS) [3] will be provided during each patient's rehabilitation program.

The general research question is:

To what extent does reduction of spasticity in the shoulder, arm and hand muscles, adjuvant to a concise arm-hand rehabilitation approach (CARAS), improve functional armhand skill performance in sub-acute post-stroke patients with a moderately to severely affected arm-hand (UAT score 1-3) and moderate to severe grades of spasticity?

This general research question is subdivided in to three specific sub-questions, to be analysed and answered using 3 design approaches. Each design approach is explained in-depth below. 
- Sub-question 1 (necessitating a single case experimental design):

Which relation exists between the time the spasticity reducing treatment was started in the sub-acute phase after stroke in subject $X$ and any changes in the time series' trend regarding each patient's arm-hand function and arm-hand skill performance?

- Sub-question 2 (necessitating a single arm group design):

To what extent does the rate of improvement as to arm-hand function and armhand skill performance change after baseline as a result of the spasticity reducing treatment in sub-acute stroke patients?

- Sub-question 3 (necessitating a non-randomised double arm group design):

To what extent do sub-acute stroke patients receiving spasticity-reducing therapy adjunct to CARAS improve more as to their arm hand function and armhand skill performance than patients who only received CARAS (i.e. patients from the AMUSE study [9])?

\section{Methods / Design}

This study (version: V2, dated June $16^{\text {th }}, 2016$ ) has received ethical approval by the Medical Ethics Committee of Maxima Medical Centre in Veldhoven, the Netherlands (METC reference number: W16.027; CCMO code: 56494.015.16). This study will be conducted according to the principles of the Declaration of Helsinki (version October 2013) and in accordance with the Dutch Medical Research Involving Human Subjects Act (Wet medisch-wetenschappelijk onderzoek met mensen (WMO) [33]).

\section{Design \& duration}

The current interventional study gauges the added-value of early post-stroke spasticity reduction in the upper extremity during 'therapy as usual', on AHF and AHSP levels in sub-acute stroke patients using three methodological approaches, i.e.:

i) A (multiple baseline) single case experimental design [34] involving 10 individuals; 
ii) A meta-analysis of the data of all single cases (= single arm group design);

iii) Non-randomised double arm group design, i.e. a case-matched control design in which each patient receiving early post-stroke spasticity reduction treatment will be matched (according to arm-hand capacity, spasticity level, age, gender, UAT score) to a case from the AMUSE study [9].

After patient's eligibility screening and written informed consent, both primary and secondary outcome measures will be taken at baseline, followed by measurements at 1-week intervals during the $2 \times 6$ weeks (total 12 weeks, i.e. CARAS training episode 1 and CARAS training episode 2) CARAS treatment and at 2-weeks intervals during the ensuing 3 months follow up, resulting in a time series per patient per outcome measure. (Figure 1). Blinding of participants, therapists or data analysts as to the intervention provided is not possible in this study.

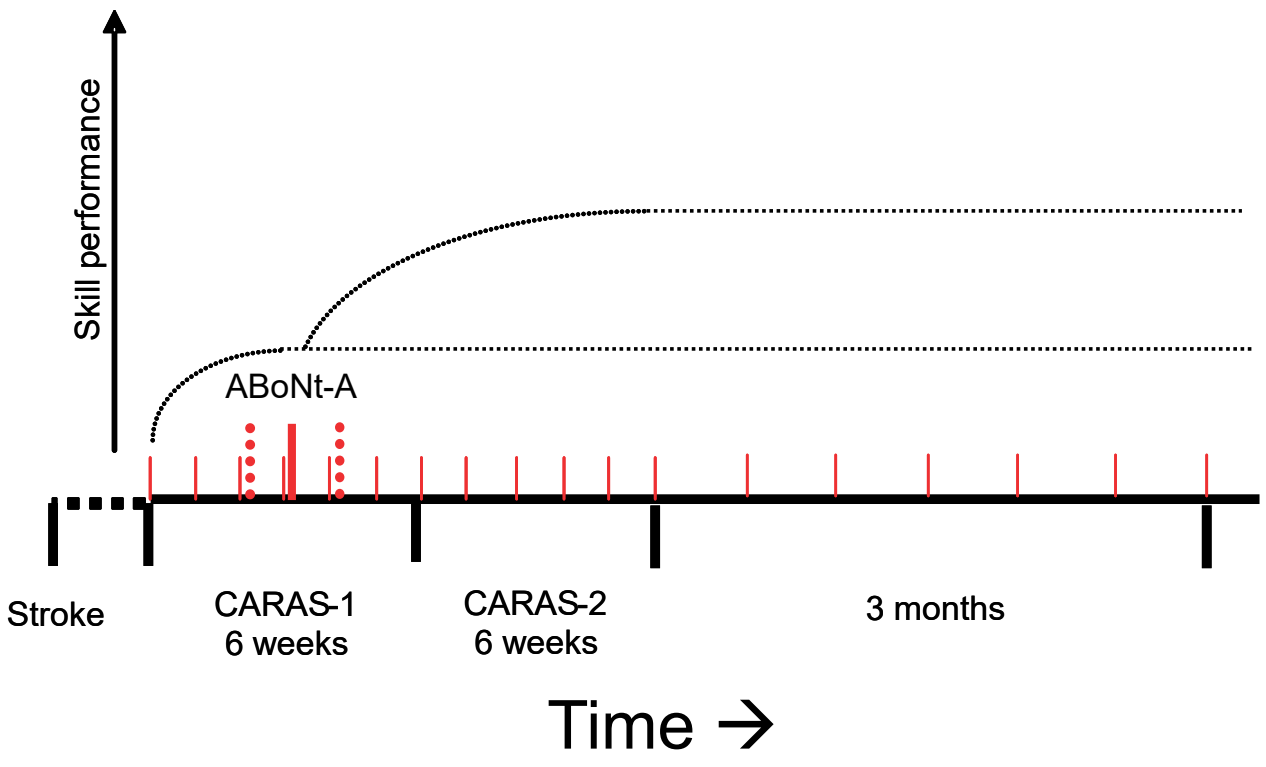

Figure 1. Measurement points in time

ABoNt-A = application of ABoNt-A. Vertical red solid lines: measurement dates. Vertical dotted lines $=$ delineation of multiple baseline, i.e. the time window in which ABoNt-A is applied. Horizontal lines = level of (postulated / expected) armhand skill performance. CARAS-1 = CARAS training episode 1. CARAS-2 = CARAS training episode 2. 
i) Multiple baseline single case experimental design:

In single case experimental design studies time series, consisting of many sequential observations (= measurements), are recorded for each individual subject. After a baseline period an experimental stimulus or intervention is applied. In the subsequent time phase the effect of this experimental stimulus or intervention on primary and secondary outcome measures relative to baseline values is investigated. The length of the baseline phase may be constant between participants (e.g. measurements may be taken weekly for 5 weeks) or may vary between participants (e.g. in subject $A$ baseline measurements may be taken weekly for 4 weeks, whereas for subject $B$ baseline measurements may be taken weekly for 5 weeks, and for subject $C$ for 6 weeks). The latter approach, featuring different starting moments of the experimental stimulus or intervention across participants in a study, is called 'multiple baseline across subjects'.

Using a 'multiple baseline' design in single case experimental research, as is done in the present study, strengthens the design [34], because it reduces the chance of any changes in the outcome parameters after the experimental stimulus of intervention being attributable to other factors than the experimental stimulus or intervention applied. In other words: If changes in outcome parameters occur ONLY AFTER the application of the experimental stimulus or intervention, the chance of this change being caused by a chance factor is reduced. We can (statistically) relate the 'position on the time axis' of the occurrence of the intervention to the 'position on the time axis' of the occurrence of any effect (=outcome measure time series trend changes). In Figure 2 an example of a multiple baseline design across subjects is depicted. 

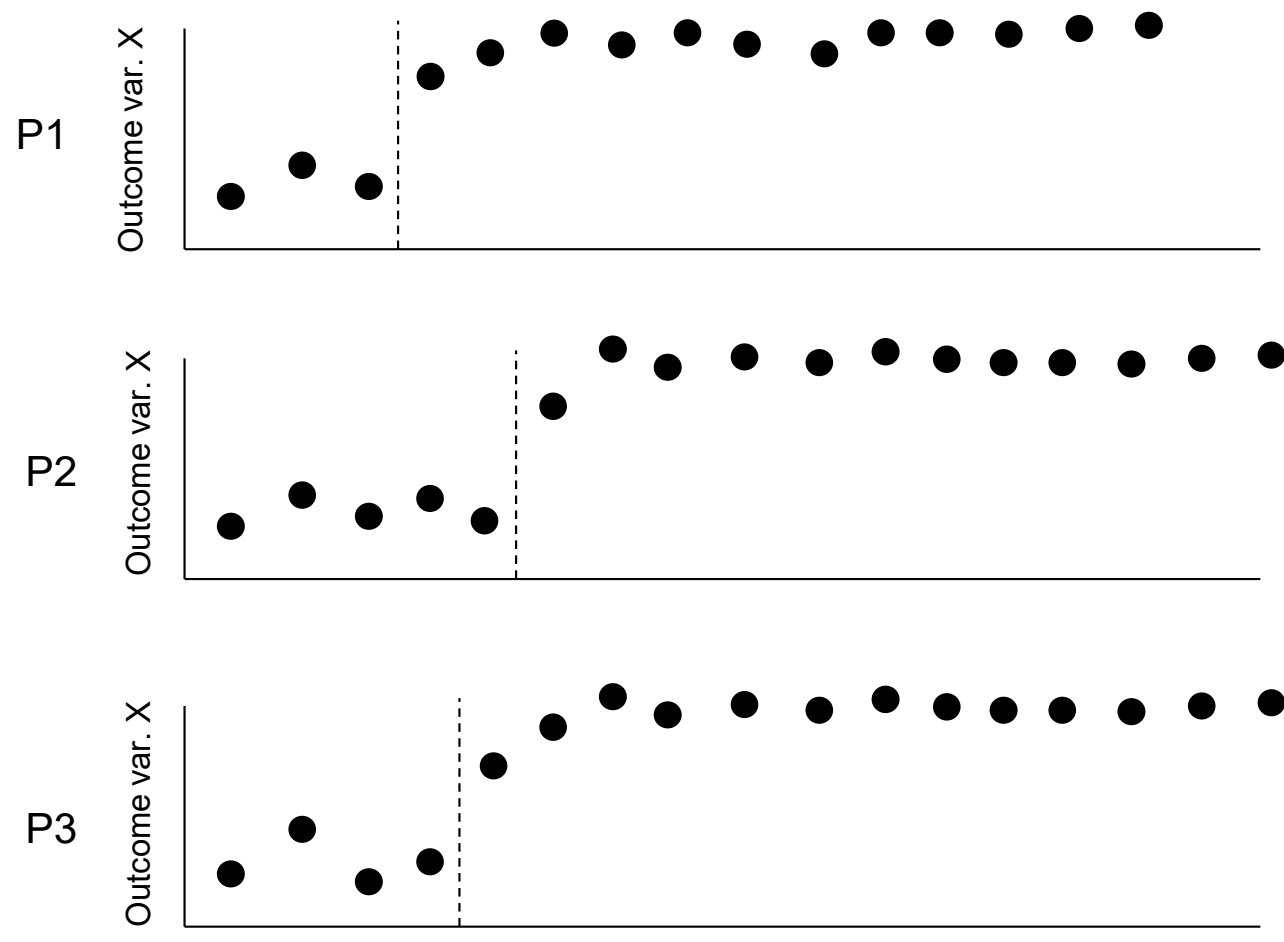

Baseline

\section{Post-intervention phase}

Figure 2. Example of a multiple baseline design across subjects. Dotted line: experimental stimulus or intervention. Dark dots: measurements of outcome variable X. P..: participants

Since the subjects in the proposed study are patients in the sub-acute phase after stroke, it is likely that their performance will improve due to e.g. spontaneous recovery and the therapy they receive, especially in the early phase post-stroke. On the other hand, the occurrence of spasticity in this sub-acute phase may slow down or hamper improvement in voluntary movements. The baseline data (i.e. the baseline time series representing changes in the outcome measures during baseline phase) thus may reflect effects of spontaneous recovery and/or effects of 'therapy as usual' (in our case CARAS). These baseline data may show a trend (e.g. towards gradual improvement of arm-hand parameter outcome over time, or e.g. a non-improvement of arm-hand parameter outcome due to spasticity starting to develop).

If, at some point, a spasticity-reducing treatment is applied, the trend of arm-hand parameter outcome observed in the prior phase (baseline phase) may change (the 
patient may become proficient more rapidly regarding outcome measure $X$ or $Y$ ). An example of this concept is given in Figure 3.

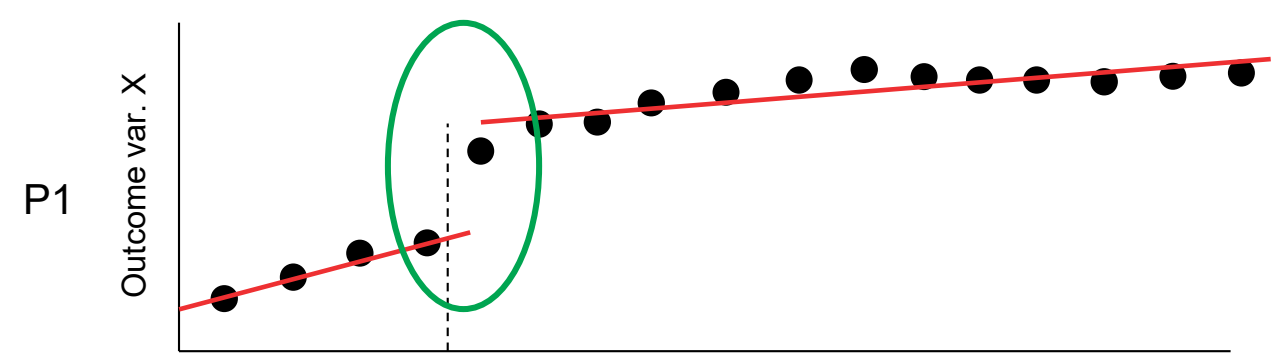

Baseline

Post-intervention phase

Figure 3. Example of change in time series trend. Dotted line: experimental stimulus or intervention. Dark dots: measurements of outcome variable X. P1: participant 1. Solid lines: baseline trend and post-intervention trend. (For simplicity reasons a linear trend line is depicted here. Other, higher order trend lines may be used.)

ii) Meta-analysis of the data of all single cases (= single arm group design)

First, each time series of each subject will be 'de-trended' for any baseline trends, similar to the approach reported by Franck et al [35]. This will result in a 'detrended' time series for each subject, data of which are rendered mutually independent by this de-trending technique. The latter is important in view of the ensuing statistics to be used. Next, for each subject, mean values of the residuals from the baseline phase and the post-intervention phase will be calculated. Subsequently, for each of the (two) phases, these data will be pooled across subjects, after which nonparametric statistical analyses will be performed on the pooled dataset.

iii)Case-matched control design (= non-randomised double arm group design)

In this design, for each of the participants in the present study, a case-matched control subject from the database of the AMUSE study will be sought. All stroke patients suffering from arm-hand problems, who are admitted to Adelante rehabilitation centre, receive CARAS treatment.

In the (previously performed) AMUSE study 89 sub-acute stroke patients who entered Adelante rehabilitation centre and who suffered from arm-hand problems 
due to the stroke, were monitored during their rehabilitation phase and up to 12 months post-discharge [9].

As to the frequency of measurements / assessment, in the AMUSE study arm-hand assessments at ICF function level and at activity level were performed at baseline (i.e. before arm-hand treatment started), at 6 weeks into the CARAS arm-hand training program, immediately after the CARAS training program (i.e. at 12 weeks post-baseline), at clinical discharge, and at 3, 6, 9 and 12 months post-discharge. Measures at ICF participation level were done at baseline, clinical discharge and at 12 months post-discharge. In order to compare data from the AMUSE study and the present study measurement, we will use data of the present study that are collected at the same time points during the AMUSE study. In Figure 4 an overview of the 'synchronous' measurement dates for both the AMUSE study and the present study are depicted.

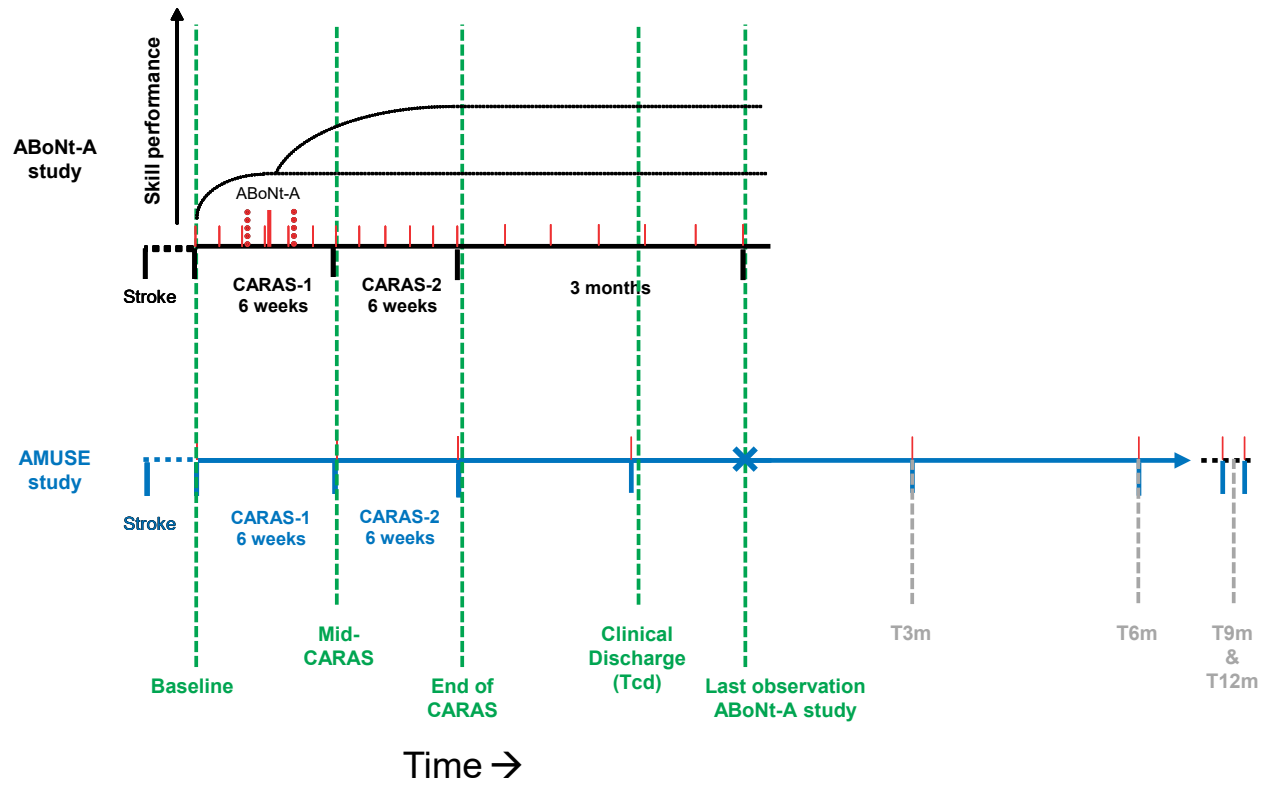

= linear interpolated AMUSE data of Tcd and T3m measurements

Figure 4. Measurement dates for both the AMUSE study and the present study. ABoNt-A = application of ABoNtA. CARAS-1 = CARAS training episode 1. CARAS-2 = CARAS training episode 2. $T=$ Measurement moment at 3 , 6, 9 and 12 months after clinical discharge. 
The AMUSE study and the present single case experimental design study share 5 similar measurement dates.

- AMUSE: baseline (Tbl)

The time at which baseline measurements are performed in the AMUSE study is identical to the time at which the first baseline measurement of the present study is performed. (Figure 4, first vertical dotted line.)

- AMUSE: mid-CARAS \& end-CARAS (T6w \& T12w)

Similarly, after the initial 6 weeks of CARAS training an arm-hand assessment has been performed in the AMUSE study (T6w or Tmid-CARAS). This measurement time is identical to one of the present study measurement dates. This also applies to the AMUSE measurement date immediately after CARAS has finished, i.e. after 12 weeks of CARAS training (T12w or T_end-of-CARAS).

- AMUSE: clinical discharge

In the AMUSE study arm-hand assessments are also performed at clinical discharge (Tcd). Again, this measurement time is identical to one of the present study measurement dates. (Figure 4, fourth vertical dotted line.)

- Last measurement date of the present study

In the AMUSE study no exact measurement date as counterpart of the last measurement of the present study is available. However, a good approximation of data from the AMUSE study at a similar time point may be performed by linearly interpolating the AMUSE data using data from Tcd and T3m, taking into account the time difference between Tcd and the time at which the last measurement of the present study was taken. (Figure $4, \mathrm{X}$-mark.)

\section{Setting}

All patients in the present study will be identified among the sub-acute stoke patient population of Adelante rehabilitation centre in Hoensbroek, the Netherlands. The present study will be performed at Adelante in Hoensbroek. All patients who, in the past, have participated in the AMUSE study (anonymous database data of which will be used in the case-matched control design part of the present study) were patients from Adelante rehabilitation centre. 


\section{Study population}

The present study focusses on adult sub-acute stroke patients with either a severely or moderately affected arm-hand (Utrecht Arm-hand Test (UAT) [7] score 1-3) and moderate to severe grades of spasticity, i.e. Modified Ashworth Scale (MAS) score 1+ to 3 .These patients will be recruited from the patient population of the department of brain injury rehabilitation of the Adelante rehabilitation centre in Hoensbroek, The Netherlands. This study will include 10 patients who develop (early signs of) spasticity in the upper extremity during the sub-acute phase after stroke.

Stroke patients who have a severe paretic arm and hand (UAT score 1-3) at admission to the rehabilitation centre will be asked to participate (by letter from their rehabilitation physician (KR)) as soon as possible after admission to the rehabilitation centre. After giving written informed consent, collected by the researcher (JAF), measurements will start according to the protocol described.

Patients, who develop early signs of spasticity in the arm and/or hand, i.e. within 5 weeks after start of arm-hand treatment (CARAS), will remain in the study. In patients who have a severe paretic arm and hand (UAT score 1-3) at admission to the

rehabilitation centre, but who do not develop early signs of spasticity within 5 weeks after start of arm-hand treatment (thereby not being in the target group), measurements to be used in the study will cease. Their rehabilitation treatment will follow 'therapy-asusual' as will their regular therapy-related clinimetrics. Any research data of the latter patient group recorded for the sole purpose of the research will be discarded/erased.

\section{Inclusion criteria}

In order to be eligible to participate in this study, a subject must meet all of the following criteria:

- Age >=18 years;

- Supratentorial stroke, i.e. arteria cerebri media infarction;

- Sub acute phase after stroke, i.e. between 2 weeks and 3 months post-stroke;

- Severe paretic arm and hand: UAT score 1-3;

- Functional disabling spasticity in the upper extremity: Modified Ashworth Scale (MAS) score 1+ to 3 (developing within 5 weeks after the start of CARAS);

- Eligible to participate in CARAS for a period of 12 weeks; 
- Being able to understand the questionnaires and measurement instructions.

As to functional disabling spasticity in the upper extremity, patients developing spasticity in the early sub-acute phase after stroke (i.e. within 5 weeks after the start of CARAS) with a Modified Ashworth Scale (MAS) score of 1+ to 3 will continue to participate in the study.

\section{Exclusion criteria}

A potential subject who meets any of the following criteria will be excluded from participation in this study:

- severe non-stroke related co-morbidity that may interfere with arm-hand function;

- additional complaints that may interfere with the execution of the measurements;

- no informed consent.

\section{Sample size calculation}

As to the single case experimental design (SCED), by definition these studies involve 1 person. Despite this, a sample size calculation is needed given the group-wise analyses of the second and third part of the present study, i.e. the meta-analysis of the data of all single cases (= single arm group design) and the case-matched control design (= non-randomised double arm group design).

The sample size calculation is based on data from the AMUSE cohort study (see also Introduction paragraph), i.e. on the improvement on the main outcome measure used in the present study (ARAT) as observed in patients with an UAT score of 1-3 who did (Group A) or did not (Group B) develop signs of spasticity in the upper extremity within the AMUSE study. In the present study the ARAT is the primary outcome measure. Mean improvement on the ARAT after 12 weeks of CARAS was 17.3 points (sd: 12.2) for Group A and 38.3 points (sd: 19.2) for group B. Given a two-sample method, a double-sided statistical test, a power of $80 \%$ and an alpha of 0.05 , a loss to follow-up of $10 \%, 10$ participants per group are needed. 
It is expected from clinical experience, our experiences with the AMUSE cohort study, and recent influx numbers of patients in Adelante rehabilitation centre that the number of 10 patients meeting the inclusion criteria within a reasonable time span (i.e. 16 months) is well-feasible. The data of 10 case-matched control subjects will be extracted from the AMUSE database. Therefore, in total, data of 20 patients will be used.

\section{Interventions}

\section{CARAS}

Arm-hand rehabilitation treatment will be provided according to the CARAS approach as described by Franck et al. [3]. In Adelante rehabilitation centre and multiple other rehabilitation centres in the Netherlands, CARAS is 'therapy-as-usual'. After a standard initial clinical assessment, patients will receive arm-hand rehabilitation treatment for $2 x$ 6 weeks. Based on the UAT scores, patients are allocated to one of three training programs, i.e.:

- CARAS program 1; severely impaired AHF subgroup (UAT=0-1).

- CARAS program 2; moderately impaired AHF subgroup (UAT=2-3).

- CARAS program 3; mildly impaired AHF subgroup (UAT=4-7).

Program 1 is titled 'taking care and prevention'. It is designed for stroke survivors who, due to the severity of the stroke, are not able to use their affected arm and hand for skill performance in daily life situations (non-functional arm-hand). Program 2 and 3 are high intensity, task-oriented arm-hand performance training programs in which patients learn to integrate their affected arm and hand in daily occupations to optimize their overall functional abilities in daily situations. In this part a distinction is made between persons who have a moderately affected arm and hand, i.e. those who are able to use their affected arm and hand for passive and active stabilisation tasks, like fixating bread while making a sandwich; and persons with a mildly affected arm and hand, who are able to use their affected arm and hand instantaneously in daily situations.

CARAS is the standard therapy (therapy-as-usual) provided by physiotherapists and occupational therapists to all stroke patients with arm-hand problems who are admitted to Adelante rehabilitation centre for treatment. This means that the decision to apply CARAS is taken before inclusion of the patient in the study.

The present study focusses on patients with initial UAT scores of 1-3. 


\section{Spasticity-reducing treatment}

In order to reduce spasticity, abobotulinum toxin-A ( $\mathrm{BBoNt}-\mathrm{A}$ ) will be administered once, i.e. directly following the sequence of baseline measurements in each patient who is developing (early signs of) spasticity in the upper extremity.

In the present study abobotulinum toxin- $\mathrm{A}$ ( $\mathrm{ABoNt}-\mathrm{A}$ ) is a non-investigational product. abobotulinum toxin selectively targets cholinergic nerve endings via binding to ectoacceptors to block acetylcholine release at the neuromuscular junction thereby abolishing the motor end-plate potential. This causes prolonged muscle weakness. Original nerve terminals regain function 12 weeks after $A B o N t-A$ injection with full recovery of nerve-evoked muscle contraction [36]. In clinical practice reduced muscle tone is seen up to 24 week in the sub-acute phase in patients with early post-stroke spasticity [37, 38].

ABoNt-A will be administered by a senior rehabilitation physician at Adelante rehabilitation centre. Patients will receive ABoNt-A injected according to clinical judgement into the dominant spastic muscles of the arm and/or forearm. The total maximum dose for the upper limb will be 1000U [39]. ABoNt-A dosage for individual muscles will be in line with the dose ranges reported by Dashtipour et al. [40], and Gracies et al. [39]. Muscles will be identified using electro stimulation or echography according to the normal practice of the clinician.

The decision to use ABoNt-A is entirely based on clinical necessity, i.e. when a patient is developing spasticity in the upper limb muscles on the affected side (MAS score 1+ to 3), as established by the rehabilitation physician. After the first finding of this increased MAS score, the decision to apply ABoNt-A will be taken by the rehabilitation physician and the application itself will be done within 1 week.

Patients in the target group, i.e. those who have a severe paretic arm and hand (UAT score 1-3) at admission to the rehabilitation centre, will be asked to participate as soon as possible after admission to the rehabilitation centre. After giving informed consent, measurements will start according to the protocol described. Therapy adherence will be monitored, based on regular clinimetric assessment, as part of the regular daily clinical practice. 


\section{Data collection \& measures used}

At the patient's entry into the study, i.e. after the eligibility screening and patient's written informed consent, the following socio-demographic variables will be recorded: age, gender, educational level and the patient's living situation. Furthermore, medical variables, obtained from medical files of the referring consultant in rehabilitation medicine, include: stroke type (haemorrhagic or ischemic), lesion site, time since stroke, paresis level, co-morbidity, hand dominance prior to the stroke, and arm- hand function status (UAT score). During the informed consent procedure, patients will be asked for permission to use this information of their medical file.

Data collection started on October $10^{\text {th }}, 2016$ when the first patient was included. Currently, patients are being recruited and enrolled.

As to the primary outcome measures, improvement of the patients' functional arm-hand skill performance capacity will be gauged by using the Action Research Arm Test (ARAT) [41-44]. The ARAT is reliable, valid and sensitive to change in patients after stroke [42, 44-46]. It consists of four subtests comprising 16 grasp movements and three reaching movements. Items are scored on a 4-point scale, with a max score of 57.

Secondary outcome measures include:

- ABILHAND [47, 48], gauging perceived level of arm-hand skill/activity proficiency. The ABILHAND is a semi-structured interview, using a 3-level ordinal rating scale: impossible (0), difficult (1), and easy (2) to perform. The ABILHAND is valid, responsive and clinically useful $[47,49]$.

- Bilateral arm activity monitoring [50, 51]: As to actual arm-hand skill performance, bilateral activity monitors (3D accelerometry, AX3, Axivity Ltd) will be worn around both wrists for a period of 4 consecutive days.

- Fugl-Meyer Motor Assessment (FMA) [44], gauging arm-hand function level. The upper extremity section of the FMA is a reliable and valid test for measuring changes in arm-hand-function in stroke patients [52, 53]. Its score ranges from 0 to 66 .

- JAMAR strength test [54], gauging muscle strength, expressed in Newton.

- Motricity Index (MI) [55], gauging functional strength measurement during performance of daily tasks.

- Modified Ashworth Scale (MAS) [32] gauging spasticity levels in the upper extremity. 
During the first 12 weeks measurements involving the ARAT, ABILHAND, FMA, JAMAR, MI and MAS will be performed weekly. In the ensuing 3 months these measures will be administered every 2 weeks. The accelerometry measurements will be performed once every 3 weeks.

\section{Data storage \& safety}

Data collected will be stored in an electronic trial master file on a secured network drive of the Adelante network. All data will be coded immediately during measurement. Coding will be done using a combination of numeric and alphanumeric characters, which are not related to the participant and cannot be used to trace/identify the participant. Non-coded data (e.g. participant's name) will be recorded separately. HAMS is the person who has sole access to the coding key. The non-coded data will solely be accessible to two persons, i.e. JAF and HAMS. The anonymised data will be accessible to JAF, RJEMS, and HAMS.

Four years after the project has finished all identifiable data will be destroyed, preventing any further link between the results and participants. All data will be destroyed and/or deleted after 15 years.

Data processing \& statistical analysis

\section{General}

For graphical data presentation, time series per subject and boxplots (for grouped data) will be used.

From the accelerometer data mean 'activity counts' during waking hours will be calculated for both hands. A 'count' is an occasion at which the accelerometer signal exceeds a predefined threshold, indicating an (arm-hand) activity intensity. An example of identifying waking hours (or 'uptime') is given in Figure 5. 


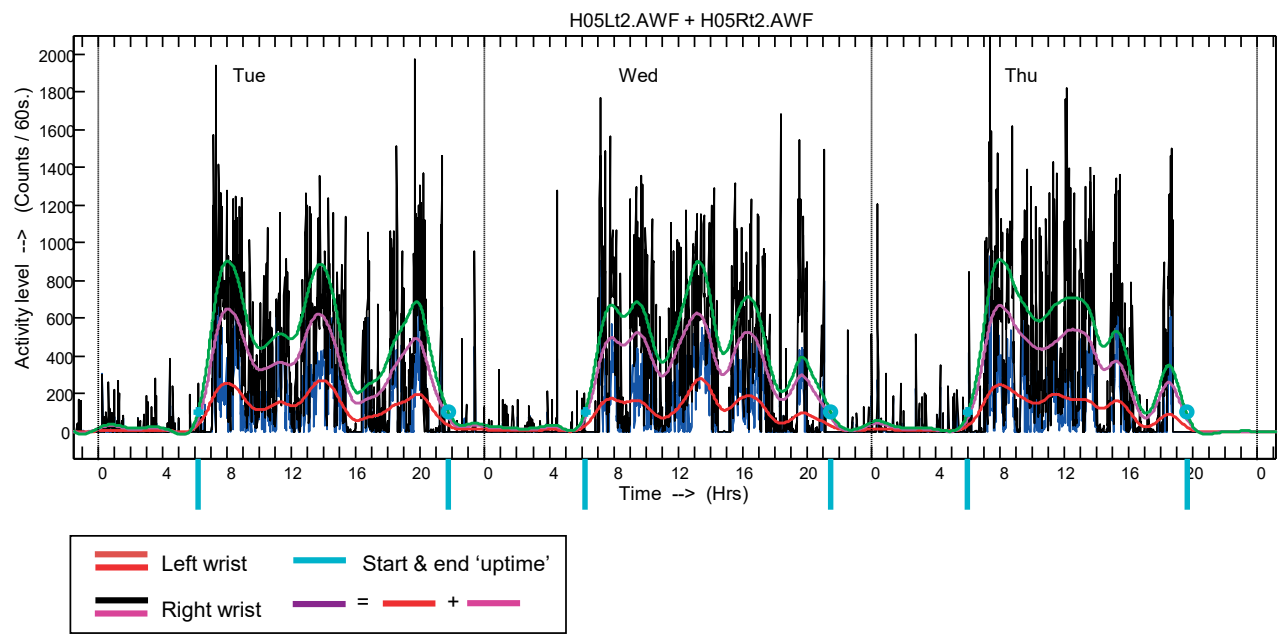

Figure 5. Identifying uptime from accelerometer signals.

For each 'uptime' the amount of time a patient used both hands, no hands, his affected hand and his unaffected hand will be calculated. Similarly, the number of 'counts' at occasions where a patient used both hands, no hands, his affected hand and his unaffected hand will be calculated. These data will each be averaged across the number of days the accelerometers were worn.

\section{Handling of missing values}

When 1 or 2 (temporally adjacent) value(s) are missing, these missing value(s) will be estimated by linear interpolation using the two valid adjacent values in the time series. In case of the final time series observation missing, the 'last-observation-carriedforward' principle will be used. In case of 3 or more consecutive missing values, the whole case (for the measure at hand) will be discarded.

\section{First design procedure: Single case experimental design}

Statistics regarding ARAT, ABILHAND, FMA, JAMAR and MI will encompass permutation tests involving the time series of each subject separately. The length of the baseline epoch will vary between 4-6 weeks between subjects. This procedure will provide an answer to the question whether there is a relation between the time the spasticity reducing treatment was started in subject $X$ and any changes in the time series' trend regarding each patient's arm-hand capacity (ARAT), each patient's arm- 
hand perceived performance (ABILHAND), as well as arm-hand function (FMA), grip strength (JAMAR) and functional arm-hand strength (MI). (Figure 3.) Here, data are analyzed for each subject separately. MAS results will be reported descriptively by plotting the time series of each subject.

Second design procedure: Meta-analysis of the data (= single arm group design) Regarding ARAT, linear detrending for any baseline trends per subject, using a least squares method, to (partially) compensate for improvements caused by e.g. spontaneous recovery and/or other treatment received, will be calculated. The time series of these residuals (data of which thus having been rendered mutually independent) of the whole group, will be further processed and analysed.

Per subject, mean residuals data of ARAT will be calculated for the baseline phase (Phase $A$ ), for the treatment phase after application of the spasticity reducing therapy (Phase B), and for the post-treatment up to 3 months post-CARAS (Phase $\mathrm{C}$ ), thus resulting in 3 mean values per subject per measure.

These mean residuals per phase will be statistically tested using non-parametric Kruskal-Wallis tests in a group design. Next, multiple comparison analysis, involving Mann-Whitney U-tests between phase A \& $C$ and phase $B$ \& $C$ will be performed using a Bonferroni correction in order to avoid spurious false positive findings. This procedure will provide an answer to the question whether, at a group level, patients improved as to their arm-hand capacity (ARAT) faster after baseline as a result of the spasticity reducing treatment.

Data processing and statistical analysis for the ABILHAND, mean 'activity counts', FMA, JAMAR, and MI will be similar to the procedures described for the ARAT. MAS results will be reported descriptively.

Third design procedure: Case-matched control design (= non-randomised double arm group design)

Finally, per subject, differences between baseline means and follow-up means will be calculated. The latter values will then be contrasted with similar values from a matched group of patients from the AMUSE study (see earlier). In this design, for each of the participants in the present study, a case-matched control subject from the database of the AMUSE study will be sought. 
In order to compare data from the AMUSE study and data from the present study measurement dates from the present study that are synchronous to the measurement dates of the AMUSE study will be identified. In Figure 4 an overview of the 'synchronous' measurement dates for both the AMUSE study and the present study are depicted.

Each subject from the SCED study part will be matched with a person from the AMUSE study according to the following procedure: Upon entry in the SCED study (baseline phase) each patient will be assessed (identical to the assessment performed in the AMUSE study subjects) in which his/her UAT score will be identified. Based on this UAT score of the SCED study subject we will search our AMUSE database for persons with an equal UAT score. Next, a match will be sought regarding the age, gender and (baseline) ARAT scores. Matching cases for spasticity level will be done when subjects first show signs of spasticity occurring, i.e. within the first 5 weeks after the start of CARAS. As an indication: About $50 \%$ of patients who have suffered an arteria cerebri media infarction and who were admitted to Adelante rehabilitation centre developed a functional limiting form of spasticity within 3-5 weeks after the start of the arm-hand training program (CARAS).

Statistical analyses of the primary outcome variable (ARAT) will include Mann-Whitney U-tests. This procedure will yield whether or not patients receiving spasticity-reducing therapy adjunct to CARAS have improved more as to their arm-hand capacity than patients who only received CARAS (i.e. patients from the AMUSE study).

Again, data processing and statistical analysis for the ABILHAND, mean 'activity counts', FMA, JAMAR, and MI will be similar to the procedures described for the ARAT. MAS results will be reported descriptively.

\section{Data reporting}

The investigators, without restrictions, will report the general, anonymous results of this study in scientific papers, and at international congresses. For all scientific reporting the guidelines of the International Committee of Medical Journal Editors (ICMJE) [56] will be adhered to. 


\section{Auditing and annual progress report}

The sponsor/investigator will submit a summary of the progress of the trial to the accredited Medical Ethics Committee (METC) once a year. Information will be provided on the date of inclusion of the first subject, numbers of subjects included and numbers of subjects that have completed the trial, serious adverse events / serious adverse reactions, other problems, and amendments. The METC exempted this study from instating a data monitoring committee. Furthermore, the sponsor/investigator adheres to Dutch law concerning any possible independent audit, at any time, by representatives of the competent national authorities.

\section{Temporary halt and (prematurely) end of study report}

The investigator/sponsor will notify the accredited METC of the end of the study within a period of 8 weeks. The end of the study is defined as the last patient's last visit. The sponsor will notify the METC immediately of a temporary halt of the study, including the reason of such an action. In case the study is ended prematurely, the sponsor will notify the accredited METC within 15 days, including the reasons for the premature termination. Within one year after the end of the study, the investigator/sponsor will submit a final study report with the results of the study, including any publications/abstracts of the study, to the accredited METC.

\section{Safety reporting}

Temporary halt for reasons of subject safety

In accordance to section 10, subsection 4, of the Dutch Person-related Research Act (WMO), the sponsor will suspend the study if there is sufficient ground that continuation of the study will jeopardise subject health or safety. The sponsor will notify the accredited METC without undue delay of a temporary halt including the reason for such an action. The study will be suspended pending a further positive decision by the accredited METC. The investigator will take care that all subjects are kept informed.

\section{Adverse events (AEs)}

Adverse events are defined as any undesirable experience occurring to a subject during the study, whether or not considered related to CARAS and/or the application of abobotulinum toxin-A. All adverse events reported spontaneously by the subject or observed by the investigator or his staff will be recorded. 
Serious adverse events (SAEs)

A serious adverse event is any untoward medical occurrence or effect that:

- $\quad$ Results in death.

- $\quad$ Is life threatening (at the time of the event).

- Requires hospitalisation or prolongation of existing inpatients' hospitalisation.

- $\quad$ Results in persistent or significant disability or incapacity.

- $\quad$ Is a congenital anomaly or birth defect.

- $\quad$ Any other important medical event that did not result in any of the outcomes listed above due to medical or surgical intervention but could have been based upon appropriate judgement by the investigator.

An elective hospital admission will not be considered as a serious adverse event. The investigator will report all SAEs to the sponsor without undue delay after obtaining knowledge of the events. The sponsor will report the SAEs through the web portal ToetsingOnline to the accredited METC that approved the protocol, within 7 days of first knowledge for SAEs that result in death or are life threatening followed by a period of maximum of 8 days to complete the initial preliminary report. All other SAEs will be reported within a period of maximum 15 days after the sponsor has first knowledge of the serious adverse events.

\section{Follow-up of adverse events}

All AEs will be followed until they have abated, or until a stable situation has been reached. Depending on the event, follow up may require additional tests or medical procedures as indicated, and/or referral to the general physician or a medical specialist. SAEs need to be reported till end of study within the Netherlands, as defined in the protocol.

\section{Amendments}

Amendments are changes made to the research after a favourable opinion by the accredited METC has been given. All amendments will be notified to the METC that gave a favourable opinion. All substantial amendments will be notified to the METC and to the competent authority. Non-substantial amendments will not be notified to the accredited METC and the competent authority, but will be recorded and filed by the sponsor. 


\section{Result and Discussion}

Given the specific research (sub-)questions, a number of issues have been taken into account in setting up this study protocol, leading to a combination of three methodological designs.

Firstly, we proposed time series analyses, i.e. a (multiple baseline) single case experimental design, in order to more individually control for spontaneous recovery and effects of therapy as usual in the sub-acute stage after stroke, which may vary considerably between patients. Modelling spontaneous recovery and effects of therapy as usual during baseline measurements has two advantages: a) the time series may be detrended for baseline trends, making any underlying mechanism to be investigated more pronounced, and $b$ ) the residuals obtained after the detrending procedure are mutually independent, thus making them eligible for statistical analyses aimed at the possible relation between the time the spasticity reducing treatment was started in the sub-acute phase after stroke in a single subject and any changes in the time series' trend regarding this patient's level of arm-hand function and arm-hand skill performance.

Secondly, pooling the averaged, baseline-detrended data of all subjects, enables group-wise data analyses gauging the (average) rate of improvement in arm-hand performance.

Thirdly, in our previous single-armed prospective cohort study, i.e. the AMUSE study [9], we obtained longitudinal data on changes in arm-hand function and arm-hand skill performance in a large group of stroke patients' typically seen in daily clinical practice. By matching patients who have received a spasticity reducing treatment with patients from the aforementioned cohort study, we can assess the added value regarding the rate of improvement in arm-hand performance that may be attributable to the spasticity reducing treatment relative to 'therapy as usual only' in sub-acute stroke patients.

The results of this study will provide evidence on the added-value of reduction of early signs of spasticity in the upper extremity on improving functional arm-hand skill performance in sub-acute stroke patients with either a severely or moderately affected arm-hand and moderate to severe grades of spasticity.

The information may lead to changes in therapy service delivery necessitating therapist to reconsider their currently used training programs. Furthermore, it may lead to further 
optimization of treatment and systematic treatment monitoring, potentially leading to better outcome of arm-hand treatment in sub-acute stroke patients.

\section{Declarations}

\section{Trial sponsor / initiator / contact}

The trial sponsor / initiator is Adelante Centre of Expertise in Rehabilitation and Audiology, Zandbergsweg 111, 6432CC, Hoensbroek, the Netherlands (www.adelante-zorggroep.nl). PI and contact person for scientific queries is HAMS, (Address: Zandbergsweg 111, 6432 CC, Hoensbroek, the Netherlands; T: +31.45.528.22.21; 1E: h.seelen@adelante-zorggroep.nl;). Contact person for public queries is JAF (Address: see above; T: +31.45.528.23.58; E: h.franck@adelantezorggroep.nl).

This trial was registered in the Dutch Trial Register (code: NTR60270) on August 4th, 2016. The secondary identifying number is: CCMO code: NL56494.015.16.

Ethics approval and consent to participate

This study (version: V2, dated June 16th, 2016) has received ethical approval by the Medical Ethics Committee of Maxima Medical Centre in Veldhoven, the Netherlands (METC reference number: W16.027; CCMO code: 56494.015.16).

\section{Consent for publication}

Not applicable.

\section{Availability of data and materials}

Not applicable. This manuscript does not contain any data.

Competing interests

Competing interests: We have the following interests: JAF, KR and HAMS are employed by Adelante. There are no patents, products in development or marketed products to declare. 


\section{Funding}

Adelante rehabilitation centre supports this study in the form of salaries of the authors JAF, KR and HAMS. This study is co-funded by Ipsen Farmaceutica BV, Hoofddorp, The Netherlands. Neither Adelante nor Ipsen Farmaceutica BV have nor will have any further role in the study design, data collection \& analysis, decision regarding publication, or preparation of the manuscript.

\section{Authors' contribution}

JAF is the main researcher in this study. HAMS, JAF and RJEMS conceptualized the study and the overall study design. HAMS elaborated the single case experimental design methodology. JAF, KR and RJEMS have provided all clinical prerequisites for the study. JAF and KR have an important role in the clinical coordination in the study. HAMS, RJEMS and JAF were involved in acquiring the project funding. HAMS and JAF perform the project administration. HAMS and RJEMS supervise the project. HAMS and JAF were involved in writing the original draft of the paper. HAMS, JAF, RJEMS and KR were involved in reviewing, editing and finalizing the paper. All authors read and approved the final manuscript. 


\section{References}

1. Langhorne P, Bernhardt J, Kwakkel G. Stroke rehabilitation. Lancet. 2011;377:1693 - 702.

2. Nijland RH, van Wegen EE, Harmeling-van der Wel BC, Kwakkel G. Presence of finger extension and shoulder abduction within 72 hours after stroke predicts functional recovery: early prediction of functional outcome after stroke: the EPOS cohort study. Stroke; a journal of cerebral circulation. 2010;41(4):745-50. Epub 2010/02/20. doi: 10.1161/strokeaha.109.572065. PubMed PMID: 20167916.

3. Franck JA, Halfens JAM, Smeets RJEM, Seelen HAM. Concise Arm and hand Rehabilitation Approach in Stroke (CARAS): A practical and evidence-based framework for clinical rehabilitation management. The Open Journal of Occupational Therapy. 2015;3(4):Article 10. doi: 10.15453/2168-6408.1164.

4. Bandura A. Self-efficacy Ramachaudran V, editor. New York: Academic Press (Reprinted in H. Friedman [Ed.],; 1994.

5. Bandura A. Self-efficacy: the exercise of control. New York: W.H Freeman and Company; 1997.

6. Lorig K, Ritter P, Stewart A, Sobel D, Brown B. Chronic disease self-management program: 2-year health status and health care utilization outcomes. Medical Care. 2001;39:1217-23.

7. Kruitwagen-van Reenen ET, Post MW, Mulder-Bouwens K, Visser-Meily JM. A simple bedside test for upper extremity impairment after stroke: validation of the Utrecht Arm/Hand Test. Disability and rehabilitation. 2009;31(16):1338-43.

8. WHO. International classification of functioning, disability and health. 2001.

9. Franck JA, Smeets RJEM, Seelen H. Changes in arm-hand function and arm-hand skill performance in patients after stroke during and after rehabilitation. PloS one. 2017;12(6):1 - 18.

10. Demetrios M, Gorelik A, Louie J, Brand C, Baquley IJ, Khan F. Outcomes of ambulantory rehabilitation programmes following botulinum toxin for spasticity in adults with stroke. $J$ Rehabil Med. 2014;46:730-7.

11. Bakheit AM, Pittock S, Moore AP, Wurker M, Otto S, Erbguth F, et al. A randomized, double-blind, placebo-controlled study of the efficacy and safety of botulinum toxin type $A$ in upper limb spasticity in patients with stroke. European Journal of Neurology. 2001;8(6):559-65.

12. Bhakta B. Management of spasticity in stroke. Br Med Bull. 2000;56(2):476-85.

13. Brashear A, Gordon M, Elovic E, Kassicieh D, Marciniak C, Do M, et al. Intramuscular injection of Botulinum Toxin for the treatment of wrist and finger spasticity after stroke. The New England Journal of Medicine. 2002;347:395 - 400. 
14. Esquenazi A, Albanese A, Chancellor M, Elovic E, Segal K. Evidence-based review and assessment of botulinum neurotoxin for the treatment of adult spasticity in the upper motor neuron syndrome. Toxicon 2013;67:115-28.

15. Foley N, Pereira S, Salter K, Fernandez M, Speechley M, Sequeira K, et al. Treatment With Botulinum Toxin Improves Upper-Extremity Function Post Stroke: A Systematic Review and Meta-Analysis. Archives of Physical Medical Rehabilitation. 2013;94:977-89.

16. McCrory P, Turner-Stokes L, Baguley I, De Graaff S, Katrak P. Botulinum toxin A for treatment of upper limb spasticity following stroke: A multicentre randomized placebocontrolled study of the effects on quality of life and other person-centred outcomes. Journal of Rehabilitation Medicine. 2009;41:536 - 44.

17. Bhakta B, Cozens J, Chamberlain M, Bamford J. Impact of botulinum toxin type A on disability and carer burden due to arm spasticity after stroke: A randomised double blind placebo controlled trial. J Neurol Neurosurg Psychiatry. 2000;69:217-21.

18. Cardoso E, Rodrigues B, Lucena R, Oliveira I, Pedreira G. Botulinum toxin type A for the treatment of the upper limb spasticity after stroke: A meta-analysis. Arq Neuropsiquiatr. 2005;63:30-3.

19. Shaw LC, Price C, van Wijck F, Shackely P, Steen N, Barnes M, et al. Botulinum Toxin for the Upper Limb After Stroke (BoTULS) Trial Effect on Impairment, Activity Limitation, and Pain. Stroke; a journal of cerebral circulation. 2011;42:1371-9.

20. Baker A, Pereira G. The efficacy of Botulinum Toxin A for limb spasticity on improving activity restriction and quality of life: a systematic review and meta-analysis using the GRADE approach. Clinical rehabilitation. 2015;30(6):549-58.

21. Prazeres A, Lira M, Aquiar P, Monteiro L, Vilasboas I, Melo A. Efficacy of physical therapy associated with botulinum toxin type $A$ on functional performance in post-stroke spasticity: A randomized, double-blinded, placebo-controlled trial. Neurology International. 2018;10(7385).

22. Wolf S, Milton B, Reiss A, Easley K, Shenvi N, Clark P. Further Assessment to Determine the Additive Effect of Botulinum Toxin Type A on an Upper Extremity Exercise Program to Enhance Function Among Individuals With Chronic Stroke but Extensor Capability. Archieves of Medical Rehabilitation 2012;93:578-87.

23. Takekawa T, Abo M, Ebihara K, Taguchi K, Sase Y, Kakuda W. Long-term effects of injection of botulinum toxin type A combined with home-based functional training for post-stroke patients with spastic upper limb hemiparesis. Acta Neurol Belg. 2013;113(4):469-75.

24. Devier D, Harnar J, Lopez L, Brashear A, Graham G. Rehabilitation plus OnabotulinumtoxinA Improves Motor Function over OnabotulinumtoxinA Alone in PostStroke Upper Limb Spasticity: A Single-Blind Randomized Trail. Toxins. 2017;9(216):1-9. 
25. Esquenazi A, Novak I, Sheean G, Singer BJ, Ward AB. International consensus statement for the use of botulinum toxin treatment in adults and children with neurological impairments--introduction. European Journal of Neurology. 2010;2:1-8.

26. Olver J, Esquenazi A, Fung V, Singer B, Ward A. Botulinum toxin assessment, intervention and aftercare for lower limb disorders of movement and muscle tone in adults: International consensus statement. Eur J Neurol. 2010;17:57-73.

27. Sunnerhagen K, Olver J, Francisco G. Assessing and treating functional impairment in poststroke spasticity. Neurol. 2013;80:35-44.

28. Turner-Stokes L, Baguley I, De Graaff S, Katrak P, Davies L. Goal attainment scaling in the evaluation of treatment of upper limb spasticity with botulinum toxin: A secondary analysis from a double-blind placebo-controlled randomized clinical trial. J Rehabil Med. 2010;42:81-9.

29. Turner-Stokes L, Fheodoroff K, Jacinto J, Maisonobe P. Results from the Upper Limb International Spasticity Study-II (ULISII): A large, international, prospective cohort study investigating practice and goal attainment following treatment with botulinum toxin $A$ in real-life clinical management. BMJ Open. 2013.

30. Turner-Stokes L, Fheodoroff K, Jacinto J, Maisonobe P, Zakine B. Upper limb international spasticity study: Rationale and protocol for a large, international, multicentre prospective cohort study investigating management and goal attainment following treatment with botulinum toxin A in real-life clinical practice. BMJ Open. 2013.

31. Demetrios M, Khan F, Turner-Stokes L, Brand C, McSweeney S. Multidisciplinary rehabilitation following botulinum toxin and other focal intramuscular treatment for poststroke spasticity. The Cochrane database of systematic reviews. 1987;(Rev 6: CD009689.).

32. Bohannon RW, Smith MB. Interrater reliability of a modified Ashworth scale of muscle spasticity. Physical therapy. 1987;67:206-7.

33. http://wetten.overheid.nl/BWBR0009408/2017-03-01.

34. Barlow D, Nock, MK, Hersen, M. Single case experimental designs:strategies for studying behavior change. Cambridge: Pearson Publishing; 2008.

35. Franck JA, Timmermans AA, Seelen HAM. Effects of a dynamic hand orthosis for functional use of the impaired upper limb in sub-acute stroke patients: A multiple single case experimental design study. Technology and Disability. 2013;25:177-87.

36. Dolly J, Aoki K. The structure and mode of action of different botulinum toxins. Eur $\mathrm{J}$ Neurol. 2006;13(1-9).

37. Rosales R, Efendy F, Teleg E, Delos Santos M, Rosales M. Botulinum toxin as early intervention for spasticity after stroke or nonprogressive brain lesion: A meta-analysis. Journal of the neurological sciences. 2016;1371:6-14. 
38. Rosales R, Kong K, Goh K, Kumthornthip W, Mok V, al. e. Botulinum toxin injection for hypertonicity of the upper extremity within 12 weeks after stroke: A randomized controlled trial. Neurorehabilitation and neural repair. 2012;26:812-21.

39. Gracies J, Brashear A, Jech R, McAllister P, Banach M, al. e. Safety and efficacy of abobotulinum toxin $A$ for hemiparesis in adults with upper limb spasticity after stroke or traumatic brain injury: A double-blind randomised controlled trial. Lancet Neurol. 2015;14:992-1001.

40. Dashtipour K, Chen J, Walker H, Lee M. Systematic Literature Review of AbobotulinumtoxinA in Clinical Trials for Adult Upper Limb Spasticity. American Journal of Physical Medicine \& Rehabilitation. 2015;94(3):229 - 38. doi: 10.1097/PHM.0000000000000208.

41. Van der Lee JH, Beckerman $\mathrm{H}$, Lankhorst GJ, Bouter LM. The responsiveness of the Action Research Arm test and the Fugl-Meyer Assessment scale in chronic stroke patients. J Rehabil Med. 2001;33(3):110-3. PubMed PMID: 11482350.

42. Van der Lee JH, De Groot V, Beckerman H, Wagenaar RC, Lankhorst GJ, Bouter LM. The intra- and interrater reliability of the action research arm test: a practical test of upper extremity function in patients with stroke. Archives of physical medicine and rehabilitation. 2001;82(1):14-9. PubMed PMID: 11239280.

43. Van der Lee JH, Roorda LD, Beckerman H, Lankhorst GJ, Bouter LM. Improving the Action Research Arm test: a unidimensional hierarchical scale. Clinical rehabilitation. 2002;16(6):646-53. PubMed PMID: 12392340.

44. Weerdt de WJG, Harrison MA. Measuring recovery of arm-hand function in stroke patients: a comparison of the Brunnstrom-Fugl-Mayer test and the Action Research Arm test. Physiotherapy Canada. 1985;37:65-70.

45. Hsieh CL, Hsueh IP, Chiang FM, Lin PH. Inter-rater reliability and validity of the action research arm test in stroke patients. Age Ageing. 1998;27(2):107-13. PubMed PMID: 16296669.

46. Yozbatiran N, Der-Yeghiaian L, Cramer SC. A standardized approach to performing the action research arm test. Neurorehabilitation and neural repair. 2008;22(1):78-90. PubMed PMID: 17704352.

47. Penta M, Tesio L, Arnould C, Zancan A, Thonnard JL. The ABILHAND questionnaire as a measure of manual ability in chronic stroke patients: Rasch-based validation and relationship to upper limb impairment. Stroke; a journal of cerebral circulation. 2001;32(7):1627-34. PubMed PMID: 11441211.

48. Penta M, Thonnard JL, Tesio L. ABILHAND: a Rasch-built measure of manual ability. Archives of physical medicine and rehabilitation. 1998;79(9):1038-42. PubMed PMID: 9749680. 
49. Ashford S, Slade M, Malaprade F, Turner-Stokes L. Evaluation of functional outcome measures for the hemiparetic upper limb: a systematic review. J Rehabil Med. 2008;40(10):787-95. PubMed PMID: 19242614.

50. Lemmens JM, Timmermans AA, Janssen-Potten YJM, Pulles SANTD, Geers RPJ, Bakx WGM, et al. Accelerometry measuring the outcome of robot-supported upper limb training in chronic stroke: a randomized controlled trail. PloS one. 2014 . doi: 10.1371/journal.pone.0096414.

51. Van der Pas S, Verbunt AJ, Breukelaar D, van Woerden R, Seelen H. Assessment of Arm Activity Using Triaxial Accelerometry in Patients With a Stroke. Archives of physical medicine and rehabilitation. 2011;92:1437 - 42.

52. Gladstone DJ, Danells CJ, Black SE. The fugl-meyer assessment of motor recovery after stroke: a critical review of its measurement properties. Neurorehabilitation and neural repair. 2002;16(3):232-40. PubMed PMID: 12234086.

53. Salter K, Jutai JW, Teasell R, Foley NC, Bitensky J, Bayley M. Issues for selection of outcome measures in stroke rehabilitation: ICF Participation. Disability and rehabilitation. 2005;27(9):507-28. PubMed PMID: 16040555.

54. Hamilton GF, McDonald C, Chenier T. Meting van de grijpkracht: waarde en betrouwbaarheid van de bloeddrukmeter en de 'Jamar grijpdynamometer'. Stimulus. 1994; Volume 13(3):164-5.

55. Wade DT. Measuring arm impairment and disability after stroke. Int Disabil Stud. 1989;11(2):89-92. PubMed PMID: 2698395.

56. http://www.icmje.org/recommendations/archives/2005_urm.pdf. 



\section{Chapter}

\section{Added-value of spasticity reduction to improve arm-hand skill performance in sub-acute stroke patients with a moderately to severely affected arm-hand}

Johan Anton Franck, Rob Johannes, Elise Marie Smeets, Jule Elmanowski, Karolien Renders, Henk Alexander Maria Seelen 


\section{Abstract}

Introduction: Spasticity is a hindrance when trying to elicit the full training potential of the affected arm-hand during rehabilitation in stroke patients with a moderately to severely affected hand. To assess to what extent arm-hand function (AHF) and arm-hand-skillperformance (AHSP) may improve by reducing early signs of spasticity in sub-acute stroke patients.

Methods: Ten sub-acute stroke patients (UAT:1-2, MAS:1+ to 3). Multiple baseline single-case-experimental-design and meta-analysis, involving 10 single cases. Training: 2x6 weeks of a well-described arm-hand regime, i.e. CARAS, including gross-motor-grip arm-hand rehabilitation. BoNT-A: administered once within the first 5 weeks. Measurement dates: weekly within the first 12 weeks; every 2-weeks during the ensuing 12 weeks. Measures: ARAT, ABILHAND, Fugl Meyer Motor Assessment, grip-strength, Motricity Index.

Results: At group level, after linear detrending, added-value of BoNT-A on AHF and AHSP could not be confirmed. Not detrended data revealed that patients improved significantly over time on AHF and AHSP on all measurements ( $p \leq 0.037)$ due to the combination of BoNT-A and CARAS.

At individual level, after baseline trend correction, adjusting for spontaneous recovery and therapy-as-usual effects, 7/10 patients improved on AHF: FM ( $N=4)(p \leq 0.019)$, grip-strength $(N=3)(p \leq 0.014)$ and $M I(N=4)(p \leq 0.002)$, whereas $6 / 10$ patients improved on AHSP: ARAT $(N=3)(p \leq 0.042)$, ABILHAND $(N=5)(p \leq 0.034)$.

Conclusion: In a number, though not all, individual patients, application of BoNT-A may have an added value in reducing early signs of spasticity. To combine a welldefined therapy-as-usual with early post-stroke spasticity reduction may improve armhand performance in sub-acute stroke patients suffering from spasticity, and who display no dexterity at the point of therapy admission. 


\section{Introduction}

The presence of spasticity in (sub-acute) stroke survivors is acknowledged as a hindrance in eliciting voluntary movement in the affected arm and hand, and may impede both arm-hand function and arm-hand skill performance enhancing interventions [1, 2]. In the present study, the term 'arm-hand function' (AHF) refers to the International Classification of Function, Disability and Health (ICF) 'body function and structures level'. The term 'arm-hand skilled performance' (AHSP) refers to the ICF activity level, covering both capacity and performance [3, 4].

Patients with a mildly impaired arm-hand have the potential or are already able to perform isolated wrist and finger movements with the affected hand and may use their affected arm-hand instantaneously in daily situations. Patients who cope with a moderately to severely affected hand at point of admission to rehabilitation show minimal abilities to generate selective arm-and hand movements. During rehabilitation, a substantial part of these patients regain a sufficient level of dexterity in order to perform gross motor grip tasks and assist the non-paretic hand during simple bimanual activities in daily life $[5,6]$. However, occurrence of moderate to severe grades of spasticity (Modified Ashworth Scale (MAS) scores 1+ to $3[7,8]$ ) in the sub-acute phase post-stroke occur more frequently in patients with a moderately to severely affected hand in contrast to patients with a mildly impaired hand [9-11].

Due to a combination of muscle weakness and spasticity in the affected arm and hand, sub-acute stroke patients with a moderately to severely affected arm-hand may be unable to attend functional rehabilitation training programs, which may lead to a delay in their functional recovery, or in failure to achieve specific treatment goals in arm hand rehabilitation $[12,13]$.

A large body of evidence on the negative influence of post-stroke spasticity on movement functions, activity performance and pain exists [14-17]. At function level, post-stroke spasticity in the affected part of the trunk, shoulder, arm and hand is associated with physiological disturbances like restricted range of joint movement, involuntary movement, a disturbed body image [1] and pain [9, 18, 19]. Problems occurring at the level of activity and participation concern, for example, hand hygiene, the application of a splint [13, 20], dressing, feeding, and sleeping. Furthermore, 
limitations in taking up societal roles, like work, family roles and leisure activities [13], as well as an increased burden on caregivers, are frequently reported [21].

Spasticity occurring in the affected arm and hand can be reduced by using botulinum toxin (BoNT) injections [2, 22]. In the past, BoNT injections were often applied as a single (pharmacological) intervention. However, effective management of reducing spasticity and enhancing hand function demands a holistic, interdisciplinary approach in which spasticity management interventions are integrated in an overall rehabilitation program [1, 20, 22-27]. Nowadays, BoNT is more frequently applied in combination with other forms of therapy, like, for instance, physical or occupational therapy [22, 26, 28]. When, adjunct to arm-hand rehabilitation interventions, BoNT is applied, one may first observe a decrease of spasticity well before improvement of AHF. During this time frame, based on motor relearning principles, patients are trained to learn how to use their upper limb muscles with reduced muscle tone within arm-hand function and armhand skill performance tasks [29].

In the past decade, a substantial number of therapy approaches were developed in which botulinum toxin was provided adjunct to therapy targeting deficits in AHF and AHSP [13, 22, 26, 28, 30-32]. However, reports on the effects of these approaches have been ambiguous $[1,18,22-24,28,33,34]$. Significant though modest results regarding active AHF after arm-hand rehabilitation combined with BoNT were reported in the systematic reviews by Foley et al. (2013) and Baker et al. (2015). The systematic review of Foley et al. (2013) contains studies featuring chronic stroke patients. The majority of studies Baker and colleagues included in their systematic review targeted sub-acute stroke populations [1, 33]. Also Takekawa et al. [25] and Devier et al. [26] demonstrated improved AHF in chronic stroke patients with a moderately to mildly impaired arm-hand who received botulinum toxin in combination with a tailored armhand rehabilitation program. However, Shaw et al. [14], Prazeres et al. [23] and Wolf et al. [24] found no added value of the injection of BoNT versus placebo both immediately followed by an arm-hand rehabilitation program with respect to AHF in chronic stroke patients. Furthermore, a recently published meta-analysis of Andringa and colleagues reported lack of effects of BoNT on arm-hand capacity [35]. The majority of the studies selected in this meta-analysis focussed on chronic stroke patients. 
The following factors may explain the ambiguity regarding the demonstration of functional improvements in AHF and AHSP after the application of arm-hand rehabilitation combined with botulinum toxin:

First, the diversity regarding (often undefined) therapy type and therapy intensity applied in conjunction with botulinum toxin across the studies [13, 33]. Sufficient details with respect to the content of additional therapy or concurrent therapy, applied in either the intervention or the control group have rarely been described in detail. Furthermore, in many cases no control group was used or the control group did not receive additional (arm-hand) therapy. For example, the control group of the study of Devier et al. did not participate in arm-hand interventions [26], and the study performed by Takakawa and colleagues did not include a control group [25]. Studies of interventions combined with botulinum toxin have tended to focus on single treatment modalities as for instance the application of electrical stimulation [36], constraint-induced movement therapy (CIMT) [37], and task-specific practice [38]. The set-up of these studies deviates from armhand interventions delivered in day-to-day arm-hand rehabilitation settings which normally consist of a complex array of interventions, adjusted to the patient's individual needs. This leads to the question whether a single intervention, combined with botulinum toxin, may be sufficient to attain relevant improvements regarding AHF and AHSP.

Secondly, the diversity in patient characteristics like post-stroke time and stroke location [9, 39, 40]: The majority of the studies published, included chronic stroke patients with a mildly to moderately affected arm-hand. One could argue that the application of an intensive arm-hand rehabilitation regime in chronic patients with a mildly impaired arm-hand may always lead to progressions. Offering a combination of intensive therapy and botulinum toxin may also lead to progressions. Demonstrating the added-value of botulinum toxin becomes even more complicated when the study population consist of sub-acute patients. In such cases it will be difficult to demonstrate the singular added-value of botulinum toxin. One way to solve the latter problem is to methodologically use a study design including a baseline trend correction, i.e. linear detrending of a time series using baseline values measured, thus correcting for both therapy-as-usual effects and spontaneous recovery effects often seen in the sub-acute phase post-stroke.

Thirdly: the different pathophysiological mechanisms leading to spasticity and how the latter affects neuromuscular control [41]. Given their biomechanical properties, 
muscles need time to change after having been injected with botulinum toxin. For example, fast glycolytic fibers often convert to slow oxidative fibers in the presence of hypertonia and time is needed for the conversion to their original glycolytic form [42]. Ultimately, this phenomenon will influence skill acquisition during arm-hand training aimed at improving daily task performance [43], and one may assume that the course of this process differs between subjects.

The most effective combination of therapy approaches, to be applied in conjunction with the application of BoNT, has not been identified yet $[13,28]$. This especially holds in sub-acute stroke patients with a moderately to severely affected arm-hand. Considering the severity of the disability that has to be overcome and in order to achieve a clinically important change in AHF and ASHP, it is essential to evaluate the patient's full potential within the limited time-window of recovery. However, the optimal type (approach, setting and modalities) and intensity of therapy to improve AHF and AHSP in this particular group of stroke patients is unclear and is often based on expert opinion only.

To structure and facilitate AHF and AHSP training in stroke survivors with a mildly affected arm-hand, but also for stroke survivors with a moderately or severely affected hand, a well-described arm and hand rehabilitation approach in stroke, called CARAS (acronym for: Concise Arm and hand Rehabilitation Approach in Stroke) [44], has been developed. Classified according to the UAT (Utrecht Arm-hand Test) scores [45], patients are allocated to one of three training programs. Program 1 is for persons with a severely impaired AHF (UAT 0-1), whereas program 2 exists out of a 'gross motor grip performance training', designed for persons with a moderately impaired AHF (UAT 2-3). Program 3 targets stroke patients with a mildly impaired AHF (UAT 4-7). Program 1 and program 3 cover a training period of six consecutive weeks. Due to their moderate level of arm-hand impairment at the initial phase of their rehabilitation period, patients admitted to program 2 participate in a 12-week during training period, consisting of 2 × 6 consecutive weeks, called 'training episode 1' and 'training episode 2', graphically presented in Figure 1.

CARAS' task-oriented training paradigm is based on stepwise clinical management procedures to elicit and define motor control problems. After having identified the motor control problems, therapists subsequently establish the most adequate set-up of exercises and motor learning approaches for the training. If done adequately, already 
in the first week of training patients may use a more efficient problem-solving strategy, leading to an easier performance of a given task.

Self-efficacy-enhancing interventions incorporated in CARAS assist patients in recognizing and monitoring improved performance, leading to successful experience, improved patient's self-efficacy, and, ultimately, improved arm-hand performance in daily activities. In CARAS, patients work towards their individual goals in groups of patients who experience similar motor impairments.

The CARAS approach provides clinicians clear rationales to assist a broad range of sub-acute stroke patients who cope with hand dexterity problems towards attaining a certain level of AHF and AHSP [44]. The development of AHF, AHSP and actual armhand use of patients who were admitted to CARAS has been well-documented and can be used as reference data $[5,46]$.

Patients with a moderately to severely affected hand show an uncertain, non-linear trend regarding arm-hand recovery $[47,48]$. In some of these patients improvements in AHF and AHSP is observed due to e.g. spontaneous recovery and the therapy they receive, especially in the early phase post-stroke $[5,6]$. Often, they undergo an armhand rehabilitation regime in order to improve on AHF and AHSP, with a limited likelihood to improve on functional tasks [1,22]. The occurrence of spasticity during this phase limits the development of voluntary movements in the affected arm and hand. Eventually, this may reduce their, already limited, possibilities to use their affected arm-hand in daily activities.

The aim of the present study is to investigate the added-value of reduction of early signs of spasticity on improving arm-hand function (AHF) and functional arm-hand skill performance (AHSP) in sub-acute stroke patients with either a severely or moderately affected arm-hand (Utrecht Arm-hand Test (UAT) [45] score 1-3) and moderate to severe grades of spasticity, i.e. Modified Ashworth Scale (MAS) score 1+ to 3 [7] adjunct to therapy-as-usual. The latter consisted of the concise arm-hand rehabilitation approach in stroke (CARAS) [44].

The research question is:

To what extent does reduction of spasticity in the shoulder, arm and hand muscles, adjuvant to a concise arm-hand rehabilitation approach (CARAS), improve arm-hand 
function and arm-hand skill performance in sub-acute post-stroke patients with a moderately to severely affected arm-hand (UAT score 1-3) and moderate to severe grades of spasticity?

\section{Methods}

The present study featured 1) a multiple baseline single case experimental design [49] involving 10 individuals in the sub-acute phase after a stroke, and 2) a meta-analysis or group analysis of the data of all these 10 single cases. To correct for improvements caused by e.g. spontaneous recovery and/or other treatment received, all-time series per subject were linearly detrended for any baseline trends. As presented in Figure 1, the study covered three phases (A, B and $C$ ) in which each participant underwent sequential observations and measurements, generating a time series per patient per outcome measure.

Measurements were repeatedly performed at baseline, with a time interval of one week. Baseline length randomly varied between 3-6 weeks across subjects during the first training period, i.e. phase $A$, in which CARAS was applied, the rationale of which has been reported by Franck et al. [44]. Consecutive to phase A, phase B started once the Abobotulinum toxin-A (ABoNt-A) was injected, which was administered adjunct to CARAS. Both phases together encompassed 12 weeks. Measurements were continued using the 1-week intervals until the end of the second 6-weeks training period (phase B). Finally, measurements performed during the ensuing 3 months follow-up (phase C) were interspaced by two weeks. A detailed description of this study protocol has been presented by Franck et al. [50]

This study received ethical approval from the Medical Ethics Committee of Maxima Medical Centre in Veldhoven, the Netherlands (METC reference number: W16.027; CCMO code: 56494.015.16). This study was conducted according to the principles of the Declaration of Helsinki (version October 2013) and in accordance with the Dutch Medical Research Involving Human Subjects Act (Wet medisch-wetenschappelijk onderzoek met mensen (WMO) [51]. 


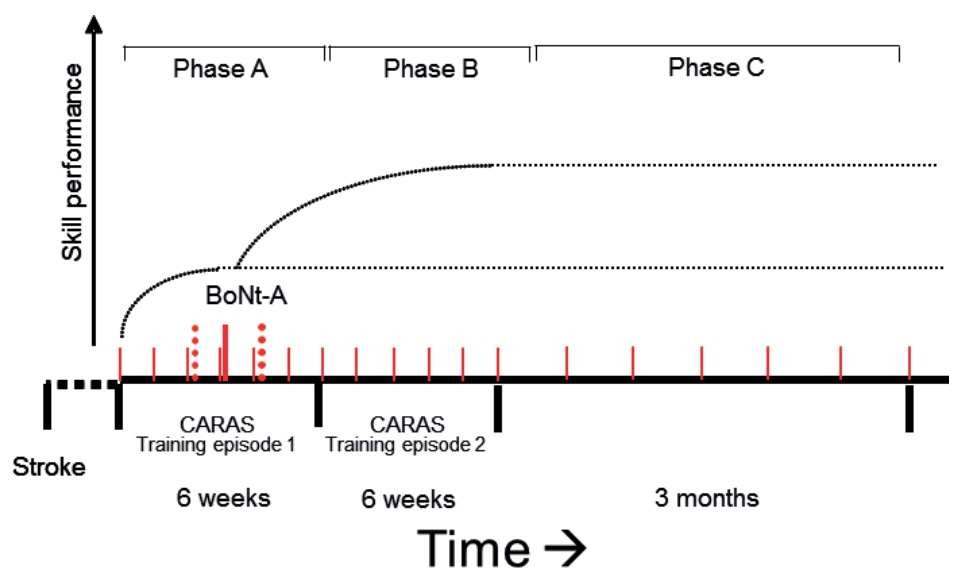

Figure 1. Schematic representation of the study design.

$A B o N t-A=$ application of $A B o N t-A$. Phase $A=3-6$ weeks of training in CARAS, program 2. Phase $B=C A R A S$ training in program 2 after ABoNt-A injections. Phase $C=$ Measurement moments from 2 till 12 weeks after CARAS. Dotted line: experimental stimulus or intervention. Dark dots: measurements of outcome variable $X$.

\section{Study population}

First, sub-acute stroke patients admitted to the department of Brain Injury Rehabilitation at Adelante rehabilitation centre in Hoensbroek, the Netherlands, were informed about the content and purpose of the study. Subsequently, they were asked to participate in this study. Written informed consent was obtained from all participants prior to the start of their participation in this study.

Patients with a moderately to severely affected arm-hand (UAT score; 1-3) who developed early signs of spasticity in the arm and/or hand, i.e. within 5 weeks after start of arm-hand treatment (CARAS), remained in the study. In patients who had a severe paretic arm and hand (UAT score 1-3) at admission to the rehabilitation centre, but who did not develop early signs of spasticity within 5 weeks after start of arm-hand treatment (thereby not being in the target group), were excluded from the study and measurements used in the study ceased. However, they continued their arm-hand rehabilitation in program 2 as 'therapy-as-usual' combined with their regular therapyrelated measurements. Any research data of the latter patient group recorded for the sole purpose of the research was discarded. 


\section{Inclusion criteria}

In order to be eligible to participate in this study, a subject had to meet all of the following criteria: Age >=18 years; stroke; sub-acute phase after stroke, i.e. between 2 weeks and 3 months post-stroke; moderate to severe paretic arm and hand (UAT score 1-3); functional disabling spasticity in the upper extremity: Modified Ashworth Scale (MAS) score 1+ to 3 (developing within 5 weeks after the start of arm-hand treatment); Eligible to participate in the CARAS program for a period of 12 weeks; Able to understand the questionnaires and measurement instructions.

\section{Exclusion criteria}

A subject who met any of the following criteria was excluded from participation in this study: severe non-stroke related co-morbidity that may interfere with arm-hand function; additional complaints that may interfere with the execution of the measurements; no informed consent.

\section{Procedures}

Patients with a moderately to severely affected arm-hand were asked to participate in the study before the start of the arm-hand treatment regime, i.e. program 2 (gross motor grip performance training) of CARAS [44]. Once admitted, measurements started according to the study protocol [50].

The training duration of CARAS' program 2 contained $2 \times 6$ weeks. A single week of training consisted of 3 days of 1.5 hours training time. All training sessions contained the following structure: Patients started with training on a personal goal for $5-10$ minutes, followed by 45 minutes of training fitted to arm-hand motor control issues, sub-goals and the patient's current performance level, which was determined by therapists prior to admission. After these 45 minutes of training, the patient worked 5 10 minutes towards his or her personal goal again. CARAS is the standard therapy (therapy-as-usual) provided by physiotherapists and occupational therapists to stroke patients with arm-hand problems who are admitted to Adelante rehabilitation centre for treatment. Once enrolled in CARAS' program 2, the patient's level of impairment and personal needs were determined, and tailored interventions were applied [44]. 
Patients who showed early signs of spasticity (MAS score $1+$ to 3 ) within the first 5 weeks of training episode 1 of CARAS were treated with ABoNt-A. The latter occurred within 1 week after the severity of spasticity was determined. Target muscles in the shoulder, arm and forearm were identified using echography. In order to avoid muscles getting excessively weakened, thereby losing their ability to facilitate movements, ABoNt-A dosages were limited to $50 \%$ of the prescribed amount related to the target muscle. [52, 53, 54].

In patients who had a moderately to severely affected arm and hand at point of admission but who did not develop spasticity (MAS score 1+ to 3) within 5 weeks after the start of the arm-hand treatment (thereby not being in the target group), measurements ceased.

\section{Outcome measures}

\section{Primary outcome measures}

Changes in patient' arm-hand skill performance capacity was measured using the Action Research Arm test (ARAT). The ARAT is a valid and reliable instrument, sensitive to change in measuring upper limb capacity at activity level in patients with stroke [55-57]. The 19 items are scored on a 4-point scale, with a total score ranging from 0 to 57.

\section{Secondary outcome measures}

Perceived performance was measured by the ABILHAND, a Rasch-analyzed test, which measures the level of manual ability in terms of the difficulty perceived by patients with hand impairments in their daily life [58]. It focuses on 23 bimanual activities that are representative for a person's daily activities [59, 60], using a 3-level ordinal rating scale: impossible (0), difficult (1), and easy (2) to perform. The ABILHAND is valid, responsive and clinically useful $[58,59]$.

At function level, the Fugl-Meyer Motor Assessment (FMA), Motricity Index (MI) [61] and JAMAR hand-held dynamometer (grip strength) were used. The FMA (part upper extremity) is a reliable and valid instrument to measure AHF in stroke patients $[62,63]$, with a score ranging from 0 to 66. The JAMAR hand-held dynamometer was used to measure grip strength of the hand (in kgf) [64]. 


\section{Data processing and statistical analysis}

\section{Handling of missing values}

When 1 or 2 (temporally adjacent) value(s) in a time series of data were missing, these missing value(s) were estimated by linear interpolation using the two valid adjacent values in the time series. In case of the final time series' observation missing, the 'lastobservation-carried-forward' principle was used.In case of 3 or more missing values, the whole case was discarded.

\section{Data analysis}

An in-depth overview of the different data analyses techniques used in this study as well as their rationale have been reported by Franck et al [50].

First, all-time series per subject were linearly detrended for any baseline trends, using a least squares method, to (partially) compensate for improvements caused by e.g. spontaneous recovery and/or other treatment received. This was done for the time series of the ARAT, ABILHAND, FMA, JAMAR and MI. The residuals, i.e. the detrended (and thereby rendered mutually independent) data, were subsequently analysed for each participant. Furthermore, mean residual data per subject per measure (FMA, MI, grip strength, ARAT and ABILHAND) were calculated for the baseline phase (Phase $A$ ), for the treatment phase after application of the spasticity reducing therapy (Phase B), and for the follow-up period (Phase $\mathrm{C}$ ). These data were analysed at group level.

\section{Group level data}

At group level, first, mean data per subject per measure (FMA, MI, grip strength, ARAT and ABILHAND) were calculated for the baseline phase (Phase $A$ ), for the treatment phase after application of the spasticity reducing therapy (Phase B), and for the followup period (Phase $\mathrm{C}$ ). Statistical (within-group) analysis of these data included Friedman two-way analysis of variance by ranks, followed by multiple comparison using Wilcoxon signed ranks tests in a Bonferroni approach. The latter was done to compensate for spurious false positive findings [65]. 
Next, all-time series per subject were linearly detrended for any baseline trends, using a least squares method, to (partially) compensate for improvements caused by e.g. spontaneous recovery and/or other treatment received. This was done for the ARAT, ABILHAND, FMA, JAMAR and MI. The residuals, i.e. the detrended (and thereby rendered mutually independent) data, were subsequently analysed at group level. Statistical (within-group) analysis of these data included Kruskal-Wallis one-way analysis of variance tests and multiple comparison involving Mann-Whitney U-tests, again in a Bonferroni approach.

\section{Individual level data}

At individual level, mean baseline trend-corrected data, i.e. the residuals, per subject per measure (FMA, MI, grip strength, ARAT and ABILHAND) for all three phases were used in the statistical analyses. The latter included Kruskal-Wallis one-way analysis of variance tests and multiple comparison involving Mann-Whitney U-tests in a Bonferroni approach.

MAS results are reported descriptively.

\section{Results}

Patient characteristics and error analysis

Thirteen patients entered the study. According to the protocol, three participants left the study within the first 5 weeks because they did not develop spasticity in the shoulder, arm or hand. No further drop-outs of study participants occurred. Ten patients (all males) completed the study. No baseline values or final follow-up values were missing. Missing values were minimal $(0.37 \%)$ and these data were estimated using linear interpolation based on the two valid adjacent values in the time series.

Two serious adverse events were reported. One patient underwent a one-day admission to the hospital because of low blood sugar levels. One patient experienced a recurrent (minor) stroke during the follow-up phase, between the $3^{\text {rd }}$ and the $4^{\text {th }}$ measurement point 
during the follow-up phase. None of these events were in any way related to the study. Due to logistical reasons, one patient was treated with ABoNt-A on the Monday of week 7 , instead of the originally planned Friday of week 6 of Phase A.

Demographic and clinical baseline data of all participants are presented in Table 1. Table 2 provides details of the muscles treated with ABoNt-A and the dosage of ABoNtA per target muscle, expressed in Units. Table 2 also presents the patients' level of spasticity as measured using the Modified Ashworth Scale in phase A, phase B and phase $\mathrm{C}$. 


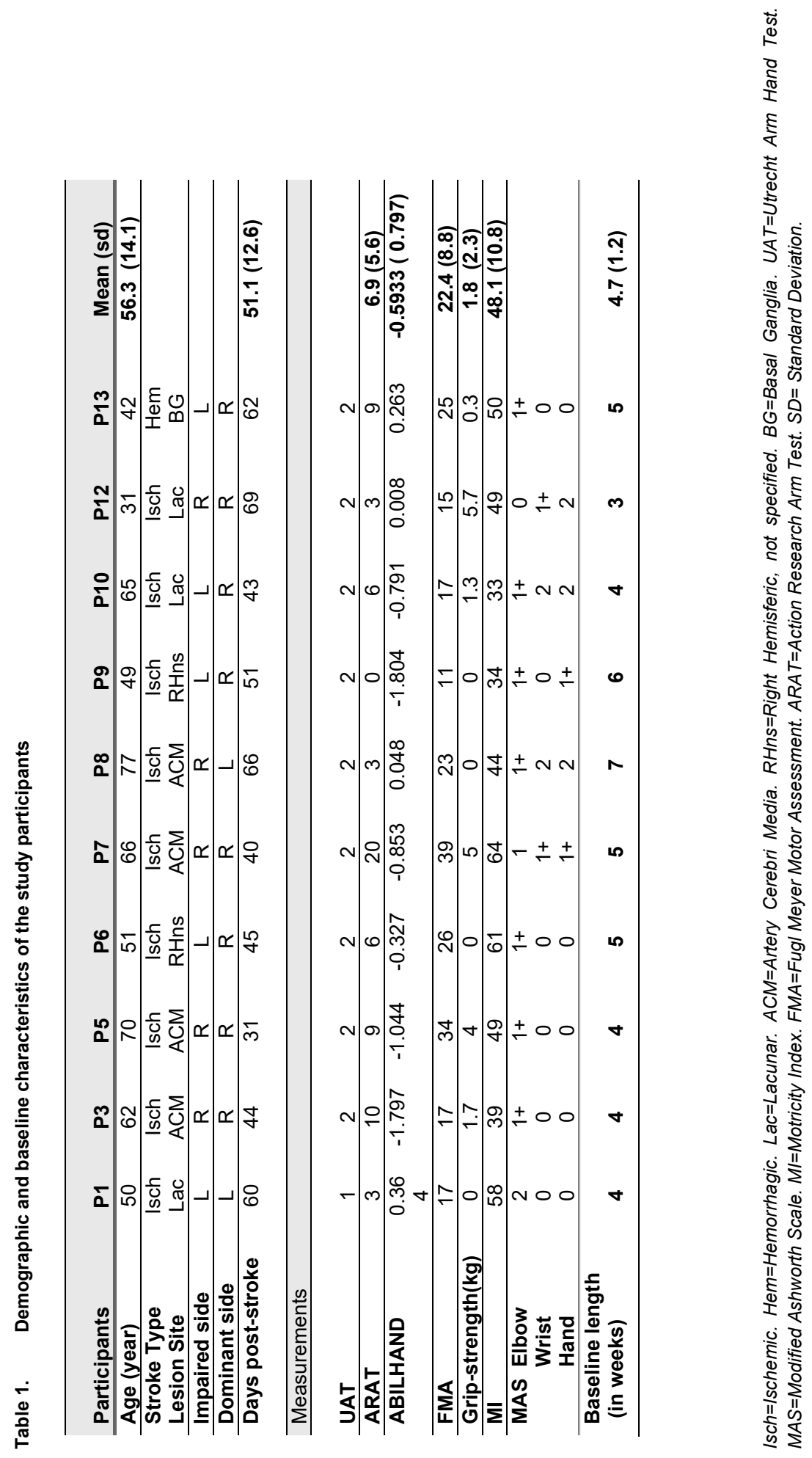




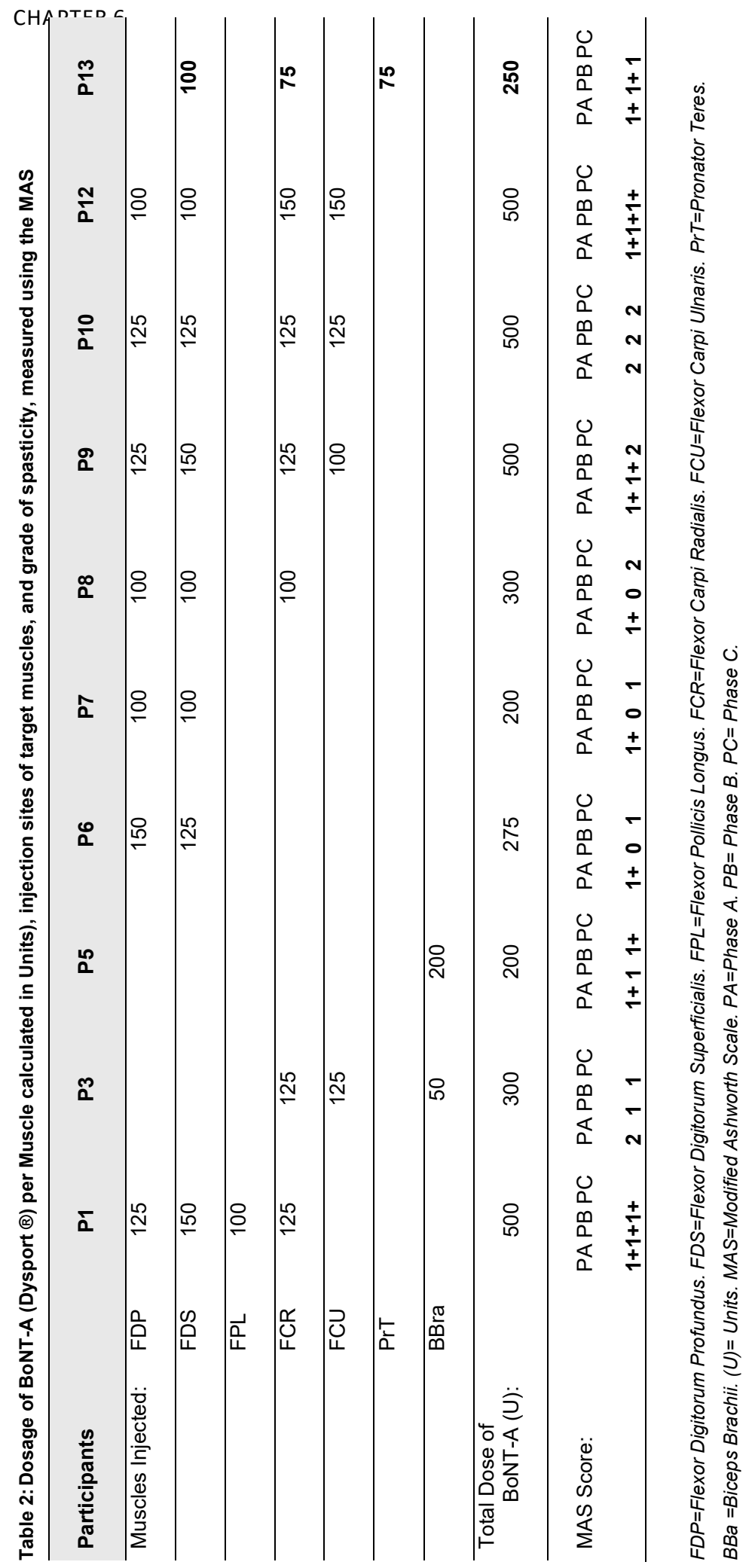


Group level data: General improvement over time

Mean group values of the ARAT, ABILHAND, FMA, grip strength and MI for all three phases, i.e. phase $A$, phase $B$ and phase $C$ are presented below.

\section{Action Research Arm Test}

Overall, on average, all patients improved over time on the ARAT ( $p=0.002)$ across all three phases. Furthermore, a multiple comparison analysis revealed that mean ARAT values were significantly improved in phase $B$, relative to the baseline data, i.e. phase $A(p=0.005)$. Also, the mean ARAT values calculated in phase $C$ were significantly higher compared to the baseline data $(p=0.013)$. No statistical differences were found for the mean ARAT data between phase $B$ and $C(p=0.221)$. Boxplots of ARAT results are presented in Figure 2.

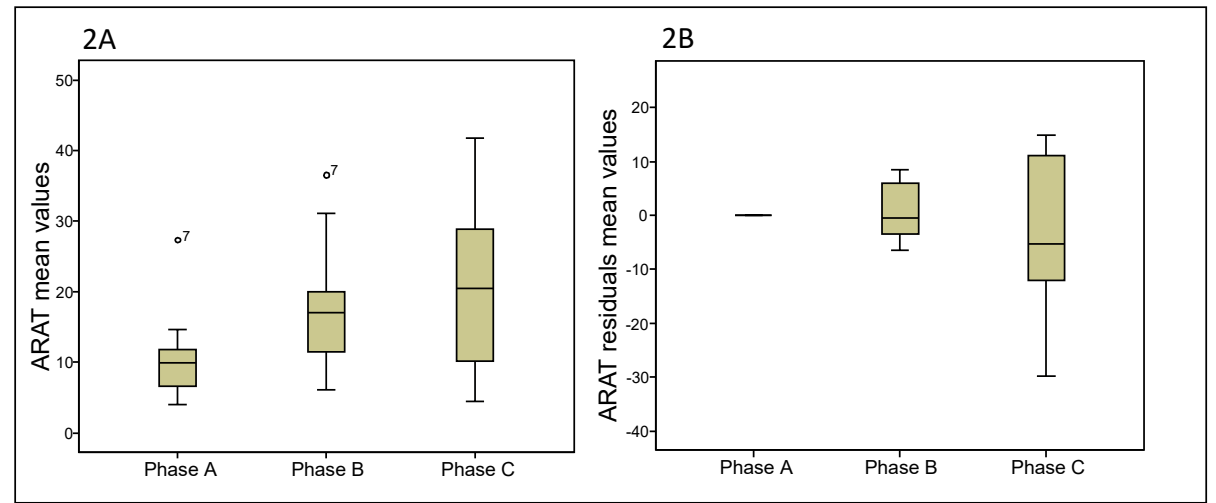

Figure 2. Boxplots of Action Research Arm Test mean values (2A) and residuals mean values (2B). ARAT = Action Research Arm Test; Circles = outlier value. 7 = measurement point 


\section{ABILHAND}

Overall, on average, patients improved over time on the ABILHAND $(p=0.001)$. Multiple comparison revealed that ABILHAND results were higher both in phase $B$ and phase $C$, relative to baseline data $(p=0.017$ and $p=0.005$ respectively). No significant differences were found between phase $B$ and $C(p=0.047)$. Boxplots of ABILHAND results are presented in Figure 3.

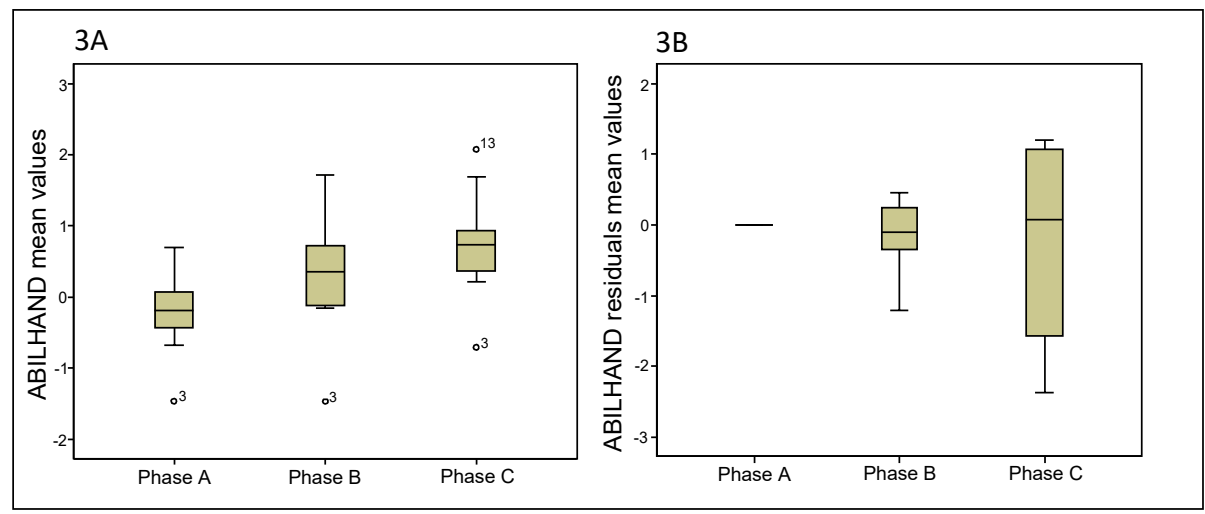

Figure 3. Boxplots of ABILHAND mean values (3A) and residuals mean values (3B). Circles = outlier value.

\section{Fugl-Meyer Motor Assessment}

Overall, on average, patients improved over time on the FMA $(p=0.001)$. Multiple comparison showed significant changes between two of the three phases; between phase $A$ and phase $B(p=0.008)$, and phase $A$ and $C(p=0.005)$. No significant differences were found between phase $B$ and $C(p=0.037)$. Boxplots of FMA results are presented in Figure 4. 


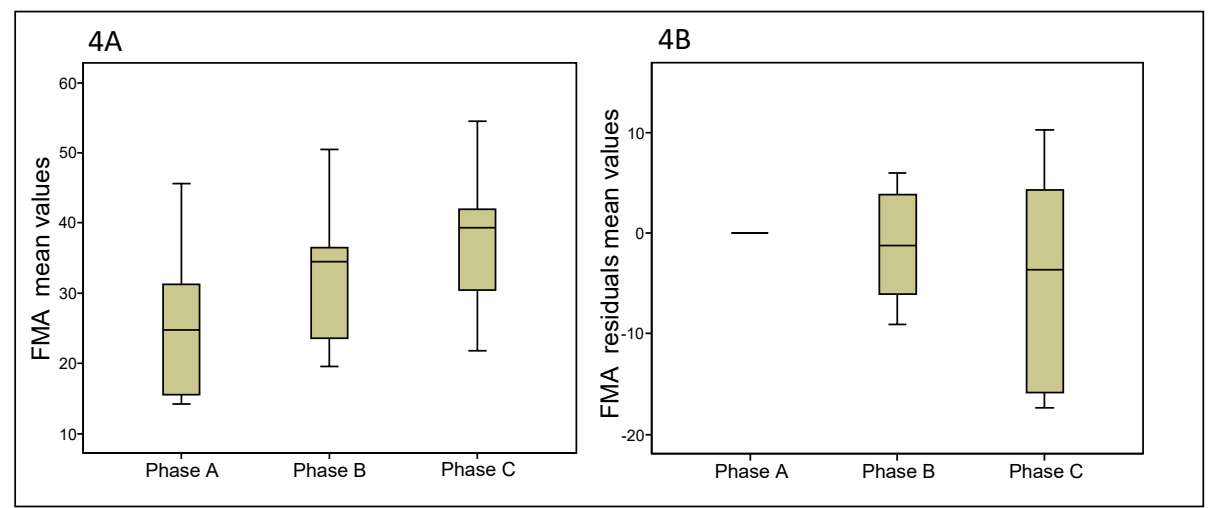

Figure 4. Boxplots of FMA mean values (4A) and residuals mean values (4B). FMA = Fugl Meyer Motor Assessment.

\section{Grip strength}

Overall, on average, patients changed significantly over time regarding grip strength $(p=0.001)$. Multiple comparison showed substantial changes between phase $B$ and phase $C(p=0.007)$ and phase $A$ and phase $C(p=0.037)$. No statistical differences were found between phase $A$ and phase $B(p=0.093)$. Boxplots of grip-strength results are presented in Figure 5.

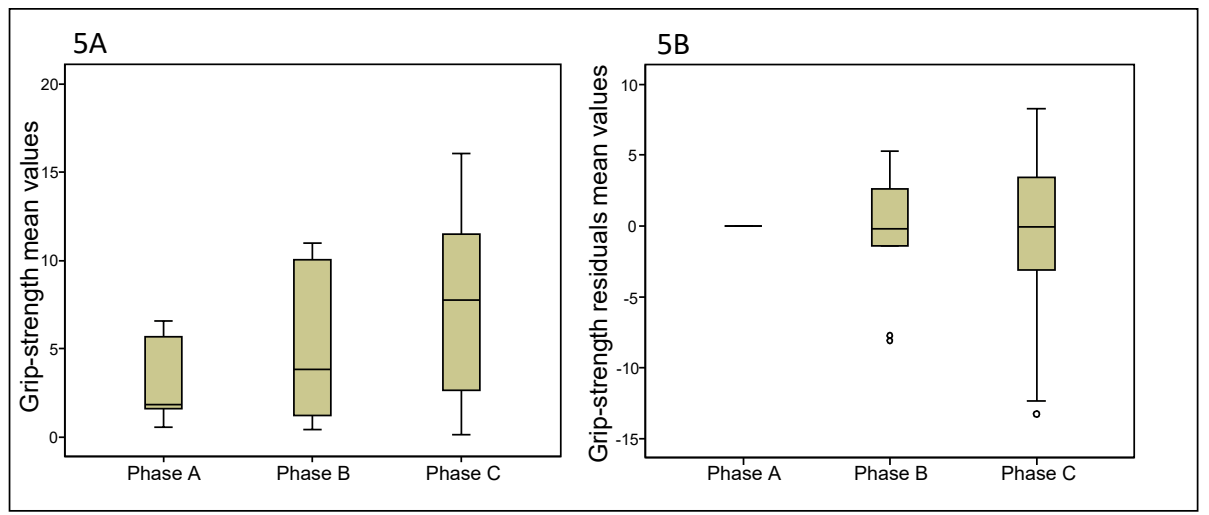

Figure 5. Boxplots of Grip strength mean values (5A) and residuals mean values (5B). Circles = outlier value. 


\section{Motricity Index}

Overall, on average, patients improved over time on strength measured using the Motricity Index $(p=0.014)$. Multiple comparison showed significant improvements between all three phases; phase $A$ and $B(p=0.013)$, phase $B$ and phase $C(p=0.028)$ and phase $A$ and phase $C(p=0.013)$. Boxplots of MI results are presented in Figure 6 .

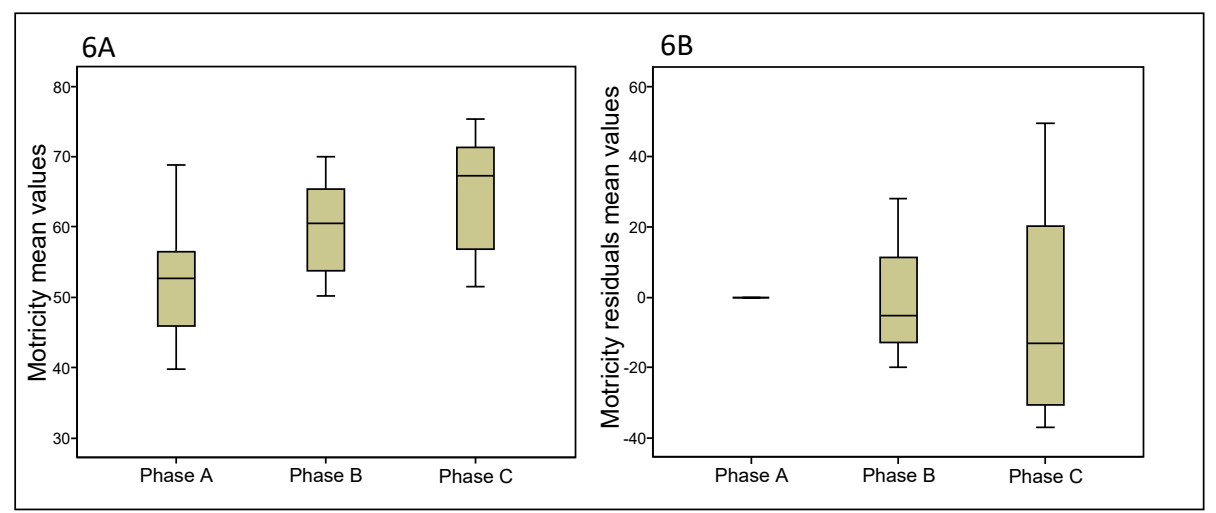

Figure 6. Boxplots of MI mean values (6A) and residuals mean values (6B). Group level data: Changes over time, corrected for baseline trends

Mean group values of the linearly baseline detrended data of the ARAT, ABILHAND, FMA, grip strength and MI, are presented above.

Boxplots of the mean linearly baseline trend-corrected ARAT, ABILHAND, FMA, grip strength and $\mathrm{Ml}$ values for all 10 participants, for all three phases, are presented in Figure $2 \mathrm{~B}, 3 \mathrm{~B}, 4 \mathrm{~B}, 5 \mathrm{~B}$ and $6 \mathrm{~B}$ respectively. No significant differences in the residuals values of the ARAT, ABILHAND, FMA, grip strength and MI were found between either one of the three phases $(p>=0.419)$. 
Individual level data: Baseline trend corrected time series for individual participants

Single case time series

Regarding changes in arm-hand capacity, for each participant, boxplots of ARAT time series residuals for phase $A, B$ and $C$ are presented in Figure 7 .

In three patients (P1, P3 and P7) overall mean ARAT residuals were higher in the follow-up phase relative to the baseline phase $(p<=0.012)$. In the remaining seven patients (P6, P8, P9, P10, P12 and P13) no statistically significant improvements in mean ARAT residuals across phases were observed. In one patient (P5) a statistically significant decrease in mean ARAT residuals was observed between phase $A$ and phase $C(p=0.002)$.

$\mathrm{P} 1$ and $\mathrm{P} 3$ showed improvements over time across phase $\mathrm{A}, \mathrm{B}$ and $\mathrm{C}$ : Residuals from phase $B$ were higher compared to phase $A$, although this difference did not attain statistical significance. In phase $C$ a significant improvement was observed relative to phase $B(p<=0.004)$. ARAT residuals calculated in phase $C$ were significantly higher than those in phase $A(p<=0.006)$. In $P 7$ results were statistically significantly different between phase $A$ and $C(p<=0.010)$. No significant differences were observed between phase $A$ and $B(p<=0.025)$. In phase $C$, in three participants, $P 1, P 9$ and $P 10$ a return of spasticity in wrist-and hand muscles was observed.

With respect to perceived arm-hand capacity (ABILHAND) and to arm-hand function (FMA, grip strength, MI) median and interquartile range of the within-subject results of all measurements are shown in Table 3a. Both the Kruskal-Wallis p-values and the subsequent multiple comparison $p$-values regarding phase $A, B$ and $C$ are presented in Table 3b. 


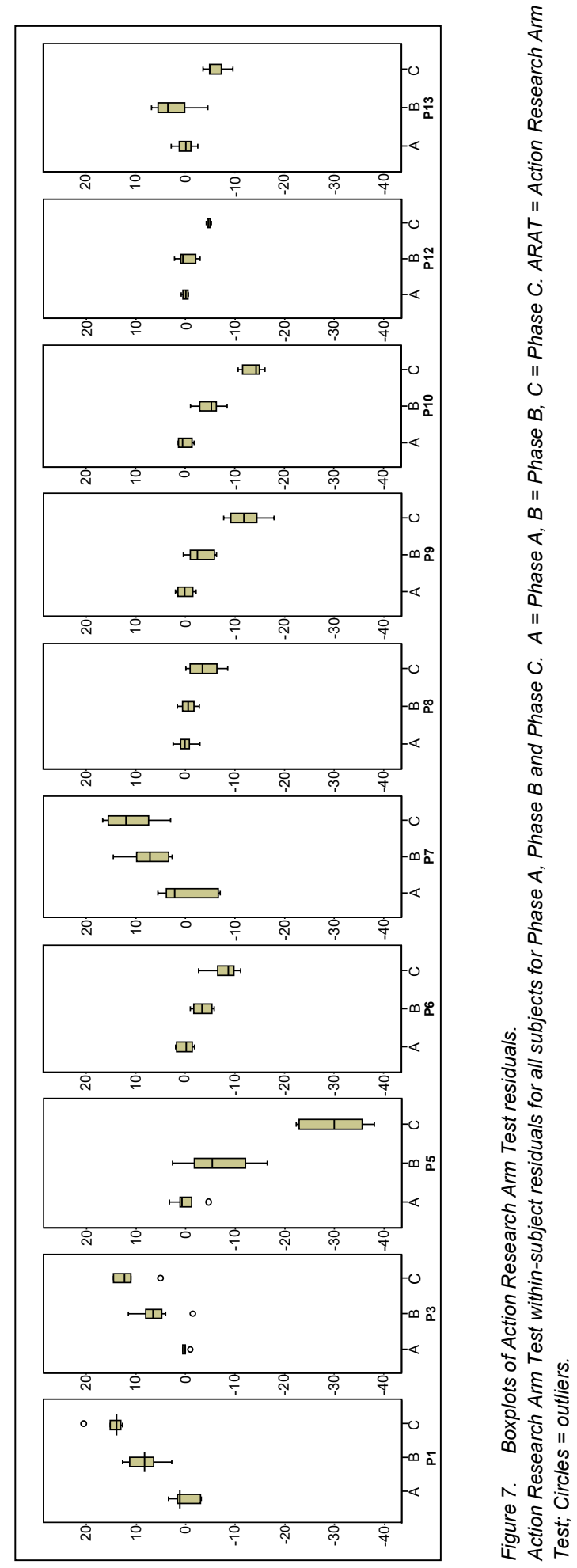




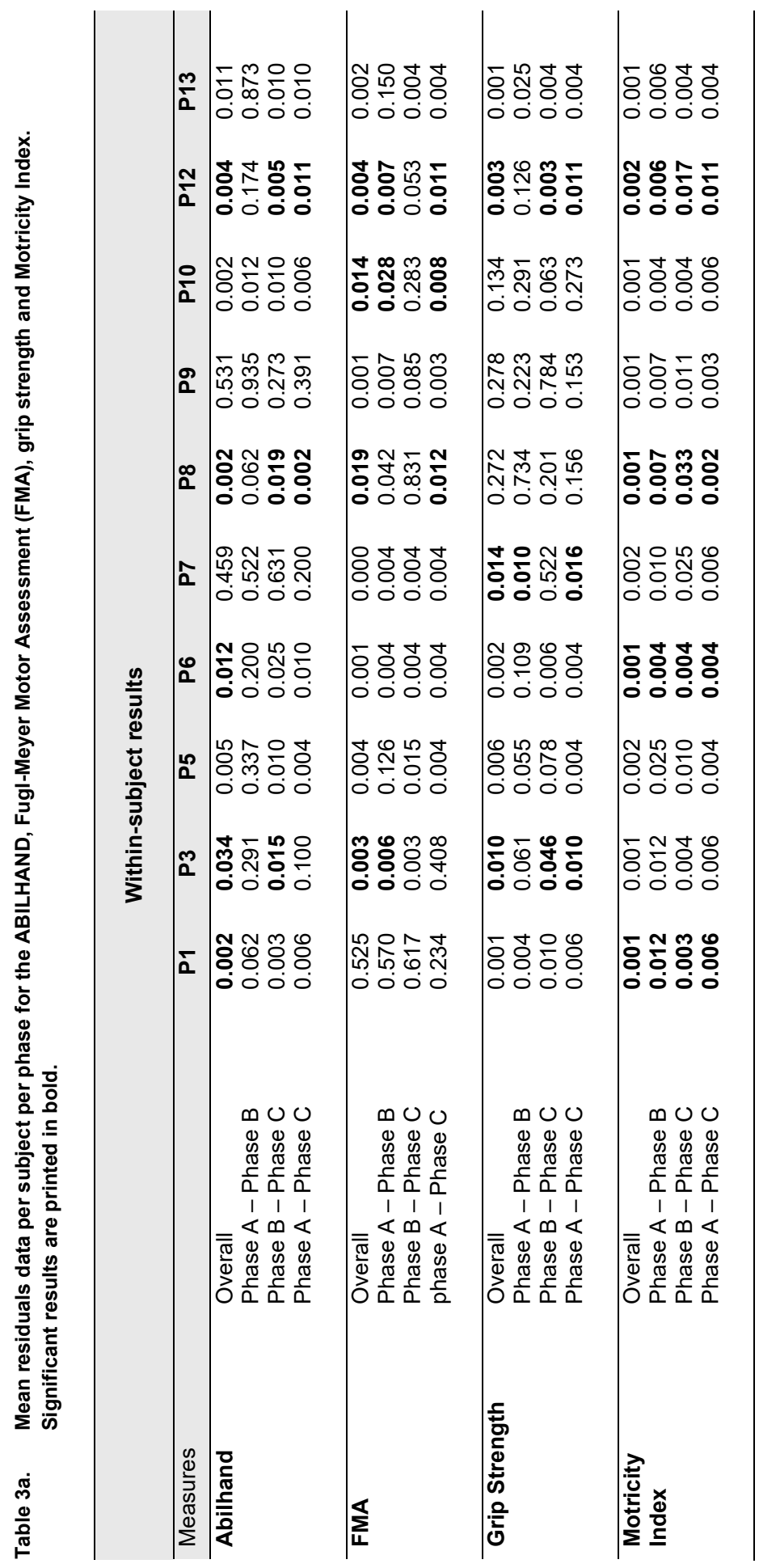




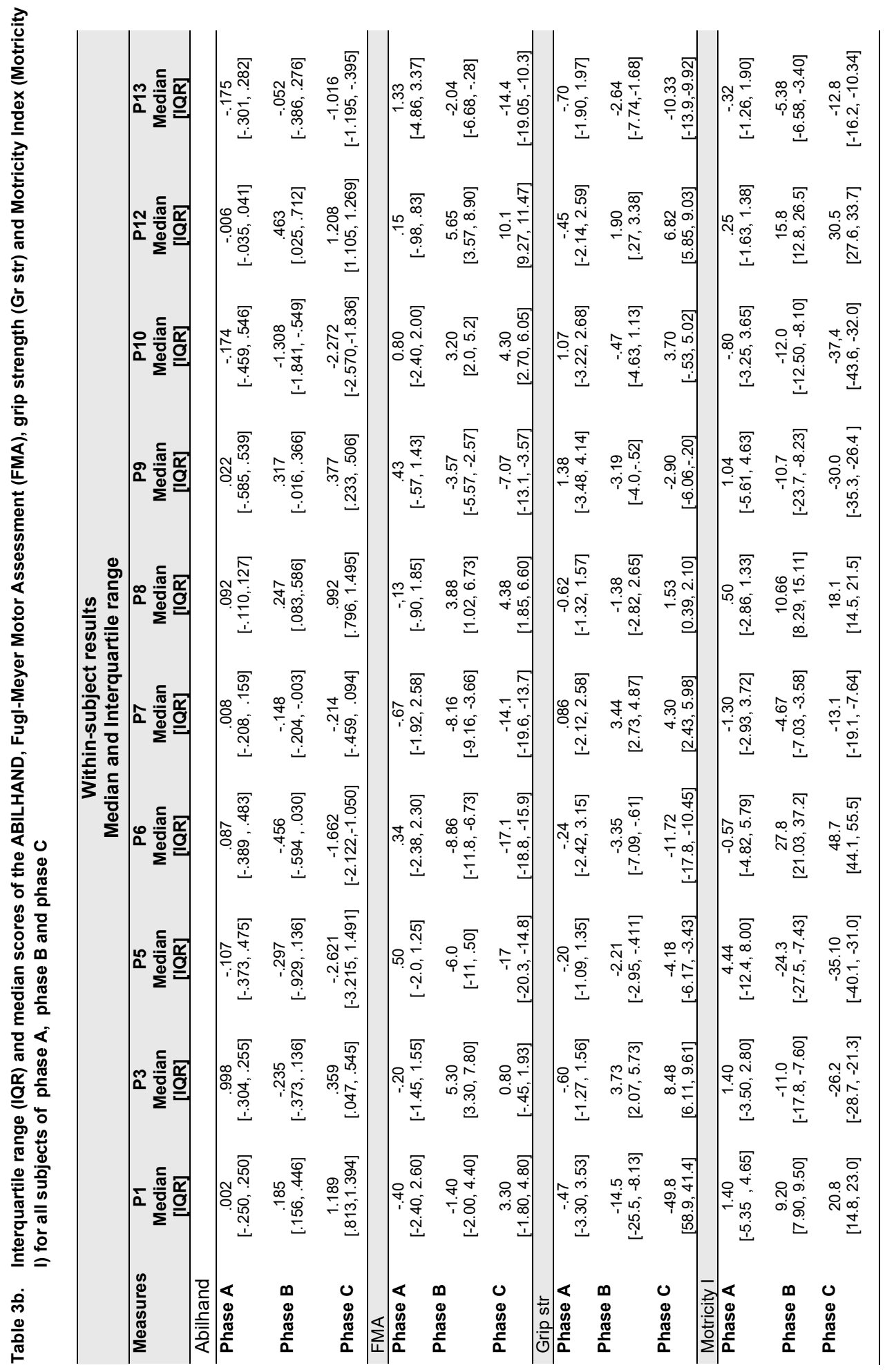




\section{Discussion}

The aim of the present study was to investigate the added-value of the reduction of early signs of spasticity on improving arm-hand function and functional arm-hand skill performance in sub-acute stroke patients with either a severely or moderately affected arm-hand (UAT score 1-3) and moderate to severe grades of spasticity, i.e. MAS scores between 1+ to 3 adjunct to a concise arm-hand rehabilitation approach [44].

To discern between spontaneous recovery and therapy-as-usual effects on the one hand, and the spasticity reducing treatment on the other hand, two methodological approaches were used. Firstly, a 'multiple baseline single experimental design' was used. Secondly, the time series of each subject were 'detrended' for any baseline trends to investigate the added-value of BoNt-A on changes in arm-hand function (AHF) and arm-hand skill performance (AHSP) in sub-acute stroke patients with a moderate to severely affected arm-hand.

At group level, on average, participants improved significantly regarding both AHF and AHSP in phase $B$ and $C$, relative to phase $A$, except for grip strength. In contrast, after baseline trend correction, data at group level did not confirm that the application of ABoNt-A resulted in an additional improvement of AHF and AHSP adjunct to therapyas-usual, i.e. CARAS. However, the application of linear detrending using the withinsubject data baseline values measured in phase A of the study may have led in some cases to a) an overestimation of spontaneous recovery and therapy-as-usual effects in phase $B$ and $C$, and could therefore have led to an underestimation of any unique effect of the ABoNt-A as applied in phase B (administration of ABoNt-A).

At individual level, as to each individual patient's baseline detrended time series, data showed that the injection of ABoNt-A in three out of the ten participants resulted in significant additional improvements in arm-hand capacity, as measured with the ARAT. Five patients improved at the level of self-perceived performance, as measured with the ABILHAND. Seven out of ten patients demonstrated significant improvements in arm-hand function (AHF). Four patients improved on motor function measured with the FMA, the Motricity Index and six patients improved on (grip)-strength. In three patients no beneficial effects from the contribution of ABoNt-A during arm-hand rehabilitation 
were observed. In one patient a decrease in ARAT residuals was observed between the trainings phase and follow-up phase.

To achieve the desired effect, i.e. a long lasting improvement of AHF and AHSP in stroke patients who suffer from spasticity, many authors recommend to apply a persontailored approach using a distinct arm-hand rehabilitation intervention in conjunction with botulinum toxin, coupled to relevant AHF and AHSP outcome measures [2, 13, $22,26,28,33,39,66]$. However, only a minority of studies actually did combine rehabilitation and the application of botulinum toxin including an arm-hand rehabilitation program which is tailored to the patients' individual characteristics and adaptable to changes in AHF and AHSP level throughout the study period. For example, Devier and colleagues combined botulinum toxin during a well described, patient-tailored arm-hand rehabilitation program. They observed AHF improvements in chronic stroke patients with a mildly affected hand [26].

Despite the fact that at group level no significant results as to the added value of $A B o N t-A$ adjunct to therapy-as-usual were found, the individual data show that several individuals showed significant improvements on AHSP and even more on AHF. With respect to these findings some remarks have to be made. First, the study participants who demonstrated higher baseline values on the Fugl Meyer wrist and hand section improved significantly at arm-hand skill capacity level during phase $B$ and/or $C$, in contrast to participants with lower initial FMA values. FMA outcome values are associated with cortico-spinal tract integrity and recovery of the affected arm-hand [67]. Our results suggest that the former subgroup may have benefitted more from spontaneous recovery in the distal part of the arm during phase $A$, compared to the latter subgroup and therefore may have obtained higher levels of arm-hand capacity during phase $\mathrm{B}$ and $\mathrm{C}$. Maybe especially in the group of patients who displayed significant improvements, the application of botulinum toxin may have facilitated the re-occurrence of voluntary movements that were hampered by spasticity before.

Secondly, the application of the linear baseline detrending of the time series may have led to an underestimation of the potential effects of the botulinum toxin in three study participants regarding both AHSP and AHF level during and after their treatment. Due to the rapidity with which spontaneous recovery combined with arm-hand training 
interventions occurred during the first (baseline) phase of rehabilitation, the linear detrending may have caused the aforementioned underestimation of any singular added value of the botulinum toxin application.

Three patients did not attain a significant arm-hand capacity level because they showed MAS scores between $1+$ and 2 in the wrist and hand combined with a low level of motor recovery in the distal part of the arm, as measured with the FMA. The combination of both a lack of strength in the wrist/hand and the presence of spasticity in wrist and hand muscles may have hampered progression at the level of arm-hand capacity.

In these three patients, who coped with a very low level of motor output in the distal part of their arm, the flexor muscles of the wrist and/or fingers were treated with ABoNtA. However, patients who received ABoNt-A in the wrist muscles and/or hand muscles may experience a temporarily delay in regaining hand function and the course of relearning how to use the affected hand. This is caused by the combination of reduced muscle tone, changes in spasticity and changes in muscle function of the already weakened wrist-and hand muscles $[29,40]$. This phenomenon, i.e. the loss of muscle function, may explain the lack of significant grip strength improvement between the baseline phase and the intervention phase in these three patients. Ultimately, this could have led to no statistically significant differences regarding arm-hand capacity being found, as a certain level of grip strength is required to observe any progression at arm-hand capacity level as measured with the ARAT.

Progression in AHF and AHSP may be hampered by co-morbidity, especially in stroke survivors with a moderately to severely affected arm-hand. Early post-stroke spasticity is highly correlated with a low motor ability level due to severe muscle weakness [29], and a high level of ADL dependency [9, 10, 68-70], shoulder pain [71, 72] or edema [73]. These symptoms were also present in the majority of the patients who participated in the present study. In order to regain control over goal-directed voluntary movements of the hand as efficiently as possible, depending on underlying sensory, motor or cognitive deficits, the aforementioned symptoms were tackled using a set-up of interventions aimed to the specific needs and abilities of each participant. However, the multitude of symptoms within a single subject affecting AHF and AHSP outcome 
to a certain extent, may have obscured the unique contribution of ABoNt-A applied in this study.

The application of botulinum toxin is considered an adjunct intervention with temporary effects [74] that provides a window of opportunity by temporarily reducing spasticity [13]. In the follow-up phase of this study, a minority of the participants experienced a return of spasticity in muscles who were previously treated with ABoNt-A, a phenomenon that negatively influenced progression regarding AHF and AHSP. Besides the temporary effect of botulinum toxin, patients with a low motor ability level experience more spasticity and associated reactions [75]. These associated reactions often appear and increase when patients become more mobile when, for example, they get out of their wheelchair and start walking longer distances. This may have led to an increase in tone and, eventually, in biomechanical and (neuro-)physiological changes of the different tissues in the affected arm-hand [17] during the training phase, thus negatively influencing AHF and AHSP.

The overall results at group level of this study are in line with Baker et al. who found significant improvements in AHSP as measured by the ARAT in sub-acute and chronic stroke patients with an affected arm-hand who received BoNT in conjunction with arm hand training [1]. Furthermore, the studies of Turner Stokes et al. (2013) and Demetrios et al. (2015) reported substantial improvements in AHF in moderately to mildly impaired chronic stroke patients who participated in an unspecified (high intensity) arm-hand rehabilitation program [13, 18]. However, at group level, the baseline trendcorrected data of our study did not confirm that the application of ABoNt-A leads to an additional improvement in moderately to severely impaired sub-acute stroke patients. This is in contrast with Cousins et al. (2010) who showed a significant positive change in AHSP after applying botulinum toxin in stroke individuals with no arm function [76]. However, in that study an undefined form of arm-hand training and botulinum toxin was provided simultaneously. This may have led to difficulties to ascertain the added-value of the botulinum toxin.

A recently published systematic review found no evidence that BoNT is effective in regaining arm-hand use [35]. The major part of this meta-analysis included studies containing chronic stroke patients. Multiple studies included in review did not offer 
adjunctive rehabilitation therapies after botulinum toxin has been applied in order to optimize voluntary control. Also information with respect to the content and dose of arm-hand therapy offered adjunctive to botulinum toxin was not clearly described. These aforementioned factors make it difficult to compare their results with our study, involving stroke patients in the sub-acute phase, who received $A B o N t-A$ and participated in a defined high-intensity arm-hand therapy regime.

Research concerning sub-acute stroke patients who suffer from a non-functional hand (UAT $0-3$ ) is scarce in literature [77, 78]. From a clinical point of view, exploring the possibilities for training methods for this particular group, especially in early post-stroke phase, is of utmost importance because this could make the difference between either no dexterity or regaining and maintaining dexterity in patients. A further study with more focus on why some individuals respond well to the combined intervention of arm-hand therapy and ABoNt-A in terms of AHF and AHSP progressions, while others do not respond well to the combined intervention, is warranted.

\section{Limitations of the study}

The present study is not without limitations. The application of linear detrending may probably have led to an underestimation of effects of the ABoNt-A applied within the arm-hand training provided in phase $B$. The latter may have been the case in at least three participants. Future research should also focus on identifying other, non-linear models that may be used to describe effects of spontaneous recovery and therapy-asusual in stroke patients, that may then be used to gauge the added value of adjunct interventions like the use of ABoNt-A.

Using the single case experimental design is a valid and efficient way to capture clinically relevant clinical questions rapidly and convert them into a research format. However, creating baseline stability regarding the dependent variables before the intervention to be investigated is applied, is difficult due to, among others, spontaneous recovery processes and therapy-as-usual offered during the baseline phase. 


\section{References}

1. Baker A, Pereira G. The efficacy of Botulinum Toxin A for limb spasticity on improving activity restriction and quality of life: a systematic review and meta-analysis using the GRADE approach. Clinical rehabilitation. 2015;30(6):549-58.

2. Sheean G, Lannin NA, Turner-Stokes L, Rawicki B, Snow BJ. Botulinum toxin assessment, intervention and after-care for upper limb hypertonicity in adults: international consensus statement. Eur J Neurol. 2010;17(2):74-93.

3. Lemmens RJ, Timmermans AA, Janssen-Potten YJ, Smeets RJ, Seelen HA. Valid and reliable instruments for arm-hand assessment at ICF activity level in persons with hemiplegia: a systematic review. BMC Neurol. 2012;12:21. Epub 2012/04/14. doi: 10.1186/1471-2377-12-21. PubMed PMID: 22498041; PubMed Central PMCID: PMCPMC3352056.

4. WHO. International classification of functioning, disability and health. 2001.

5. Franck JA, Smeets RJEM, Seelen H. Changes in arm-hand function and arm-hand skill performance in patients after stroke during and after rehabilitation. PloS one. 2017;12(6):1 $-18$.

6. Hayward KS, Kuys SS, Barker RN, Brauer SG. Can stroke survivors with severe upper arm disability achieve a clinically important change in arm function during inpatient rehabilitation? A multicentre, prospective, observational study. NeuroRehabilitation. 2014;35(1):17-23. Epub 2014/07/06. doi: 10.3233/nre-141096. PubMed PMID: 24990005.

7. Ashworth B. Preliminary trial of carisoprodol in multiple sclerosis. Partictioner. 1964;192:540-2.

8. Bohannon RW, Smith MB. Interrater reliability of a modified Ashworth scale of muscle spasticity. Physical therapy. 1987;67:206-7.

9. Wissel J, Verrier M, Simpson D, Charles D, Guinto P, Papapetropoulos S, et al. Poststroke spasticity: predictors of early development and considerations for therapeutic intervention. Physical medicine and Rehabilitation. 2015;7:60 - 7.

10. Lundstrom E, Smits A, Terent A, Borg J. Time-course and determinants of spasticity during the first six months following first-ever stroke. J Rehabil Med. 2010;42:296 - 301.

11. Sunnerhagen KS. Predictors of spasticity after stroke. Curr Phys Med Rehabil Rep. 2016;4:182 - 5.

12. Esquenazi A, Mayer N. Botulinum toxin for the management of muscle overactivity and spasticity after stroke. Current atherosclerosis reports. 2004;3:295-8.

13. Demetrios M, Gorelik A, Louie J, Brand C, Baquley IJ, Khan F. Outcomes of ambulantory rehabilitation programmes following botulinum toxin for spasticity in adults with stroke. $\mathrm{J}$ Rehabil Med. 2014;46:730-7. 
14. Shaw LC, Price C, van Wijck F, Shackely P, Steen N, Barnes M, et al. Botulinum Toxin for the Upper Limb After Stroke (BoTULS) Trial Effect on Impairment, Activity Limitation, and Pain. Stroke; a journal of cerebral circulation. 2011;42:1371-9.

15. Brashear A, Gordon M, Elovic E, Kassicieh D, Marciniak C, Do M, et al. Intramuscular injection of Botulinum Toxin for the treatment of wrist and finger spasticity after stroke. The New England Journal of Medicine. 2002;347:395 - 400.

16. Baricich A, Picelli A, Molteni F. Post-stroke spasticity as a condition: a new perspective on patient evaluation. Funct Neurol. 2016;31:179-80.

17. Sheean G. Botulinum treatment of spasticity: why is it so difficult to show a functional benefit? Current opinion in neurology. 2001;14:771-6.

18. Turner-Stokes L, Fheodoroff K, Jacinto J, Maisonobe P. Results from the Upper Limb International Spasticity Study-II (ULISII): a large, international, prospective cohort study investigating practice and goal attainment following treatment with botulinum toxin $A$ in real-life clinical management. BMJ Open. 2013;3.

19. Yelnik A, Colle F, Bonan I, Vicaut E. Treatment of shoulder pain in spastic hemiplegia by reducing spasticity of the subscapular muscle: a randomised, double blind, placebo controlled study of botulinum toxin A. J Neurol Neurosurg Psychiatry. 2007;78:845-8.

20. Bhakta B. Management of spasticity in stroke. Br Med Bull. 2000;56(2):476-85.

21. Lundstrom E, Terent A, Borg J. Prevalence of disabling spasticity 1 year after first-ever stroke. Eur J Neurol. 2008;15:533-9.

22. Royal College of Physicians BSoRM, The Chartered Society of Physiotherapy, Association of Chartered Physiotherapists in Neurology and the Royal College of Occupational Therapists. Spasticty in adults: management of botulinum toxin national guidelines 2018 London: RCP; 2018.

23. Prazeres A, Lira M, Aquiar P, Monteiro L, Vilasboas I, Melo A. Efficacy of physical therapy associated with botulinum toxin type $A$ on functional performance in post-stroke spasticity: A randomized, double-blinded, placebo-controlled trial. Neurology International. 2018;10(7385).

24. Wolf S, Milton B, Reiss A, Easley K, Shenvi N, Clark P. Further Assessment to Determine the Additive Effect of Botulinum Toxin Type A on an Upper Extremity Exercise Program to Enhance Function Among Individuals With Chronic Stroke but Extensor Capability. Archives of medical rehabilitation 2012;93:578-87.

25. Takekawa T, Abo M, Ebihara K, Taguchi K, Sase Y, Kakuda W. Long-term effects of injection of botulinum toxin type A combined with home-based functional training for post-stroke patients with spastic upper limb hemiparesis. Acta Neurol Belg. 2013;113(4):469-75. 
26. Devier D, Harnar J, Lopez L, Brashear A, Graham G. Rehabilitation plus OnabotulinumtoxinA Improves Motor Function over OnabotulinumtoxinA Alone in PostStroke Upper Limb Spasticity: A Single-Blind Randomized Trail. Toxins. 2017;9(216):1-9.

27. Esquenazi A, Novak I, Sheean G, Singer BJ, Ward AB. International consensus statement for the use of botulinum toxin treatment in adults and children with neurological impairments--introduction. European Journal of Neurology. 2010;2:1-8.

28. Kinnear B, Lannin N, Cusick A, Harvey L, Rawicki B. Rehabilitation Therapies After Botulinum Toxin-A Injection to Manage Limb Spasticity: a Systematic Review. Physical therapy. 2014;94:1569-81.

29. Francis HP, Wade DT, Turner-Stokes L, Kingswell RS, Dott CS, Coxon EA. Does reducing spasticity translate into functional benefit? An exploratory meta-analysis. Journal of Neurology Neurosurgery Psychiatry. 2004;75:1547 - 51.

30. Monaghan K, Horgan F, Blake C, Cornall C, Hickey PPM, Lyons BE, et al. Physical treatment interventions for managing spasticity after stroke. Cochrane Database of Systematic Reviews. 2011;(7).

31. Kuo CL, Hu GC. Post-stroke spasticity: A Review of Epidemiology, Pathophysiology, and Treatments. International Journal of Gerontology. 2018:1-5.

32. Ward AB, Wissel J, Borg J, Ertzgaart P, Herrmann C, Kulkarni J, et al. Functional goal achievement in post-stroke spasticity patients: the botox economic spasticity trail (BEST). Journal of Rehabilitation Medicine. 2014;46:504-13.

33. Foley N, Pereira S, Salter K, Fernandez M, Speechley M, Sequeira K, et al. Treatment With Botulinum Toxin Improves Upper-Extremity Function Post Stroke: A Systematic Review and Meta-Analysis. Archives of Physical Medical Rehabilitation. 2013;94:977-89.

34. Dong $\mathrm{Y}, \mathrm{Wu} \mathrm{T}$, Xiaohua $\mathrm{H}$, Wang $\mathrm{T}$. Efficacy and safety of botulinum toxin type A for upper limb spasticity after stroke or traumatic brain injury: a systematic review with meta-analysis and trial sequential analysis. European journal of physical and rehabilitation medicine. 2017;53(2):256-67.

35. Andringa A, Port I, van Wegen E, Ket J, Meskers C, Kwakkel G. Effectiveness of Botulinum Toxin Treatment for Upper Limb Spasticity Poststroke Over Different ICF Domains: a Systematic Review and Meta-Analysis. Archives of physical medicine and rehabilitation. 2019. doi: doi.org/10.1016/j.apmr.2019.01.016.

36. Hesse S, Reiter F, Konrad M, Jahnke M. Botulinum toxin type A and short-term electrical stimulation in the treatment of upper limb flexor spasticity after stroke: a randomized, double-blind, placebocontrolled trail. Clinical rehabilitation. 1998;(12):381-8.

37. Sun SF, Hsu CW, Sun HP, Hwang CW, Yang CL, Wang JL. Combined botulinum toxin type A with modified constraint-induced movement therapy for chronic stroke patients with 
upper extremity spasticity: a randomized controlled study. Neurorehabilitation and neural repair. 2010;24:34-41.

38. Weber DJ, Skidmore ER, Niyonkuru C, Chang CL, Huber LM, Munin MC. Cyclic functional electrical stimulation does not enhance gains in hand grasp function when used as an adjunct to onabotulinumtoxin $A$ and task practice therapy: a single-blind, randomized controlled pilot study. Archives of Physical Medical Rehabilitation. 2010;91:679-86.

39. Picelli A, Tamburin S, Gajofatto F, Zanette G, Praitano M, Saltuari L, et al. Association between Severe Upper Limb Spasticity and Brain Lesion Location in Stroke Patients. Biomed Research International. 2014:1-6.

40. Fransisco G. Botulinum Toxin for Post-stroke Spastic Hypertonia: A Review of its Efficacy and Application in Clinical Practice. Ann Acad Med Singapore. 2007;36:22-30.

41. Lieber R, Roberts T, Blemker S, Lee S, Herzorg W. Skeletal muscle mechanics, energetics and plasticity. Journal of neuroengineering and rehabilitation. 2017;14(108):1-16.

42. Edwards S. Physiotherapy management of established spasticity. In: Sheean G, editor. Spasticity Rehabilitation. London: Churchill Communications Ltd; 1998.

43. Carr J, Shephard R. Stroke Rehabilitation. Guidelines for exercise and training to optimize motor skill. Edinburgh: Butterworth Heinemann. Elsevier Science Limited.; 2003. pp301.

44. Franck JA, Halfens JAM, Smeets RJEM, Seelen HAM. Concise Arm and hand Rehabilitation Approach in Stroke (CARAS): A practical and evidence-based framework for clinical rehabilitation management.The Open Journal of Occupational Therapy. 2015;3(4):Article 10. doi: 10.15453/2168-6408.1164.

45. Kruitwagen-van Reenen ET, Post MW, Mulder-Bouwens K, Visser-Meily JM. A simple bedside test for upper extremity impairment after stroke: validation of the Utrecht Arm/Hand Test. Disability and rehabilitation. 2009;31(16):1338-43.

46. Franck JA, Smeets RJEM, Seelen HAM. Changes in actual arm-hand use in stroke patients during and after clinical rehabilitation involving a well-defined arm-hand rehabilitation program: A prospective cohort study. PloS one. 2019:1-22. doi: https://doi.org/10.1371/journal.pone.0214651

47. Prabhakaran S, Zarahn E, Riley C, Speizer A, Chong JY, Lazar RM, et al. Inter-individual variability in the capacity for motor recovery after ischemic stroke. Neurorehabilitation and neural repair. 2008;22(1):64-71. Epub 2007/08/10. doi: 10.1177/1545968307305302. PubMed PMID: 17687024.

48. Winters C, Kwakkel G, Nijland R, van Wegen E. When Does Return of Voluntary Finger Extension Occur Post-Stroke? A Prospective Cohort Study. PloS one. 2016;11(8):1-12. doi: 10.1371/journal.pone.0160528. 
49. Barlow D, Nock, MK, Hersen, M. Single case experimental designs: strategies for studying behavior change. Cambridge: Pearson Publishing; 2008.

50. Franck JA, Smeets RJEM, Renders K, Seelen HAM. Added-value of Early Post-stroke Spasticity Reduction during Arm-hand Rehabilitation in Improving Functional Arm-hand Skill Performance: A Multiple Baseline Single Case Experimental Design Study. Internation Journal of Neurorehabilitation. 2018;5(4).

51. Nederlandse_Rijksoverheid_[Dutch_Government]. http://wetten.overheid.nl/BWBR0009408/2017-03-01

52. Bakheit AM, Pittock S, Moore AP, Wurker M, Otto S, Erbguth F, et al. A randomized, double-blind, placebo-controlled study of the efficacy and safety of botulinum toxin type $A$ in upper limb spasticity in patients with stroke. European Journal of Neurology. 2001;8(6):559-65.

53. Suputtitada A, Suwanwela NC. The lowest effective dose of botulinum A toxin in adult patients with upper limb spasticity. Disability and rehabilitation. 2005;27:176-84.

54. Ipsen F. Summary of product characteristics. Dysport RVG 175052016.

55. Hsieh CL, Hsueh IP, Chiang FM, Lin PH. Inter-rater reliability and validity of the action research arm test in stroke patients. Age Ageing. 1998;27(2):107-13. PubMed PMID: 16296669.

56. Yozbatiran N, Der-Yeghiaian L, Cramer SC. A standardized approach to performing the action research arm test. Neurorehabilitation and neural repair. 2008;22(1):78-90. PubMed PMID: 17704352.

57. van der Lee JH, Roorda LD, Beckerman H, Lankhorst GJ, Bouter LM. Improving the Action Research Arm test: a unidimensional hierarchical scale. Clinical rehabilitation. 2002;16(6):646-53. PubMed PMID: 12392340.

58. Ashford S, Slade M, Malaprade F, Turner-Stokes L. Evaluation of functional outcome measures for the hemiparetic upper limb: a systematic review. J Rehabil Med. 2008;40(10):787-95. PubMed PMID: 19242614.

59. Penta M, Tesio L, Arnould C, Zancan A, Thonnard JL. The ABILHAND questionnaire as a measure of manual ability in chronic stroke patients: Rasch-based validation and relationship to upper limb impairment. Stroke; a journal of cerebral circulation. 2001;32(7):1627-34. PubMed PMID: 11441211.

60. Penta M, Thonnard JL, Tesio L. ABILHAND: a Rasch-built measure of manual ability. Archives of physical medicine and rehabilitation. 1998;79(9):1038-42. PubMed PMID: 9749680.

61. Demeurisse G, Demol O, Robaye E. Motor evaluation in vascular hemiplegia. European Neurology. 1980;19(6):382-9. 
62. Gladstone DJ, Danells CJ, Black SE. The fugl-meyer assessment of motor recovery after stroke: a critical review of its measurement properties. Neurorehabilitation and neural repair. 2002;16(3):232-40. PubMed PMID: 12234086.

63. Salter KL, Teasell RW, Foley NC, Jutai JW. Outcome assessment in randomized controlled trials of stroke rehabilitation. Am J Phys Med Rehabil. 2007;86(12):1007-12. PubMed PMID: 17912137.

64. Hamilton GF, McDonald C, Chenier T. Meting van de grijpkracht: waarde en betrouwbaarheid van de bloeddrukmeter en de 'Jamar grijpdynamometer'. Stimulus. 1994; Volume 13(3):164-5.

65. Siegel S, Castellan N. Nonparametric statistics. New York: McGraw-Hill International Editions; 1988. 399 p.

66. Mills PB, Finlayson H, Sudol M, O'Connor R. Systematic review of adjunct therapies to improve outcomes following botulinum toxin injection for treatment of limb spasticity. Clinical rehabilitation. 2016;30(6).

67. Jang SH, Kim YH, Cho SH, Lee JH, Park JW, Kwon YH. Cortical reorganization induced by task-oriented training in chronic hemiplegic stroke patients. Neuroreport. 2003;14(1):137-41. PubMed PMID: 12544845.

68. Leathley MJ, Gregson JM, Moore AP, Smith TL, Sharma AK, Watkins CL. Predicting spasticity after stroke in those surviving to 12 months. Clinical rehabilitation. 2004;18:438-43.

69. Opheim A, Danielsson A, Alt Murphy M, Persson HC, Sunnerhagen KS. Early prediction of long-term upper limb spasticity after stroke: Part of the SALGOT study. Neurology. 2015;85:873-80.

70. Pundik S, McCabe J, Skelly M, Tatsuika C, Daly J. Association of spasticity and motor dysfunction in chronic stroke. Annals of physical and rehabilitation medicine. 2018;1207:1-6.

71. Lindgren I, Jonsson AC, Norrving B, Lindgren A. Shoulder pain after stroke: a prospective population-based study. Stroke; a journal of cerebral circulation. 2007;38(2):343-8.

72. Ratnasabapathy $Y$, Broad J, Baskett J, Pledgern M, Marshallm J, Bonita R. Shoulder pain in people with a stroke: a population-based study. Clinical rehabilitation. 2003;17:304-11.

73. Boomkamp-Koppen HG, Visser-Meily JM, Post MW, Prevo AJ. poststroke hand swelling and oedema: prevalence and relationship with impairment and disability. Clinical rehabilitation. 2005;19(5):552-9.

74. De Paiva A, Meuniere F, Molgo J, Aoki K, Dolly J. Functional repair of motor endplates after botulinum neurotoxin type A poisoning: biphasic switch of synaptic activity between nerve sprouts and their parent terminals. J Proc Natl Acad Sci. 1999;96:3200-5.

75. Bhimani R, Anderson L. Clinical Understanding of Spasticity: Implications for Practice. Rehabilitation Research and Practice. 2014;2014:1-10. 


\section{CHAPTER 6}

76. Cousins E, Ward A, Roffe C, Rimington L, Pandyan A. Does low-dose botulinum toxin help the recovery of arm function when given early after stroke? A phase II randomized controlled pilot study to estimate effect size. Clinical rehabilitation. 2010;24:501-13.

77. Hayward K, Barker R, Brauer S. Interventions to promote upper limb recovery in stroke survivors with severe paresis: a systematic review. Disability and rehabilitation. 2010;32(24):1973-86. PubMed PMID: 20964563.

78. Oujamaa L, Relave I, Froger J, Mottet D, Pelissier JY. Rehabilitation of arm function after stroke. Literature review. Annals of physical and rehabilitation medicine. 2009;52(3):26993. PubMed PMID: 19398398. 


\section{Chapter}

\section{Evaluation of a functional hand orthosis combined with}

electrical stimulation adjunct to arm-hand rehabilitation in sub-acute stroke patients with a severely to moderately affected hand function

Johan Anton Franck, Rob Johannes Elise Marie Smeets, Henk Alexander Maria Seelen 


\section{Abstract}

Purpose: To investigate the usability and effectiveness of a functional hand orthosis, combined with electrical stimulation adjunct to therapy-as-usual, on functional use of the moderately/severely impaired hand in sub-acute stroke patients.

Materials and Methods: Single case experiment (A-B-A'-design) involving eight subacute stroke patients. The functional hand orthosis and electrical stimulation were used for six weeks, four days/week, 45\%/day. Outcome measures: Action Research Arm Test, Intrinsic Motivation Inventory.

Results: At group level, patients improved 19.2 points (median value) (interquartile range: $[8.8,29.5]$ points) on the Action Research Arm Test ( $p=0.001)$. After correcting for spontaneous recovery and/or therapy-as-usual effects Action Research Arm Test scores still improved significantly (median: 17.2 points; interquartile range: [5.1, 29.2] points) $(p=0.002)$. At individual level, six patients had improved as to arm-hand skill performance at follow-up ( $p<=0.010)$. In one patient arm-hand skill performance improvement did not attain statistical significance. In another patient no arm-hand skill performance improvement was observed. Average Intrinsic Motivation Inventory subscores were between 4.6 and 6.3 (maximum: 7), except for 'perceived pressure/tension' (3.3).

Conclusion: Sub-acute stroke patients who display only little/modest improvement on their capacity to perform daily activities, seem to benefit from training with a dynamic arm orthosis in combination with electrical stimulation. Patients' perceived intrinsic motivation and sense of self-regulation was high. 


\section{Introduction}

Stroke leaves approximately $50 \%$ of its survivors disabled with regard to arm-hand performance, often for the rest of their lives [1, 2]. An important treatment aim in stroke rehabilitation is to achieve and maintain dexterity, i.e. a clinically relevant improvement in both arm-hand function and arm-hand skill performance. The term 'arm-hand function' (AHF) refers to the 'body function and structure level' of the International Classification of Functioning, Disability and Health (ICF) [3]. The term 'arm-hand skilled performance' (AHSP) refers to the ICF activity level, covering both capacity and performance' [4]. Selecting the optimal therapy from a wide range of arm-hand interventions is challenging and depends on, among others, the severity of the AHF impairment and the potential (spontaneous) recovery of the affected arm-hand. These aspects may vary considerably among patients $[5,6]$. In order to simplify this heterogeneity problem concerning arm-hand impairment, a stratification may be used in which dexterity can be roughly classified into three levels, i.e. a mildly, moderately or severely impaired arm-hand, [7, 8]. For this classification, the Utrecht Arm hand Test (UAT) can be used [9].

The present study is focused on patients who suffer from a moderately to severely affected arm-hand (UAT score 1 or 2). These patients are insufficiently able to generate voluntary movements to participate in regular arm-hand skill training at the start of rehabilitation [10, 11], and are often excluded from clinical effectiveness studies. Especially in the early sub-acute phase after stroke, i.e. between 24 hours till 3 months post-stroke [12], this inability to participate in regular arm-hand skill training may hamper improvement in AHF and AHSP. Within this timespan patients with a severely impaired arm-hand show an uncertain, non-linear trend as to recovery $[13,14]$ and are often labeled beforehand as having 'low potential' regarding recovery of arm-hand function.

Next to traditional treatment methods for patients with a moderate to severe arm-hand paresis [5, 15], training applications also incorporating assistive devices, like robot assisted therapy with electrical stimulation [16] or dynamic orthoses fitted to the affected arm-hand to facilitate participation in task-oriented training, are emerging [17, 18]. In contrast to the growing body of evidence regarding the effectiveness of such specific interventions in persons with a moderately to mildly affected arm-hand $[5,19$, 
20], studies into the effectiveness of interventions featuring combinations of taskoriented training methods and assistive devices for the most severely affected group of sub-acute stroke patients are still scarce [21], and the effectiveness of many assistive devices in stroke rehabilitation is still equivocal [22, 23]. However, modest results as to the use of dynamic hand orthoses combined with task-oriented training in moderately to severely affected stroke patients have been reported [17, 24-26]. Stroke patients who are able to regain wrist and finger extension have sufficient residual voluntary movement ability to work independently with these types of orthoses. However, due to absence of voluntary muscle contraction towards extension and flexion of the wrist and fingers, patients will be unable to execute grasp movements independently.

The application of electrical stimulation, adjuvant to a dynamic orthosis, to provoke normal muscle activation of the fingers while the orthosis keeps the paretic wrist and hand in an optimal position, has already been considered by others [18, 20]. It is assumed that these devices may assist patients with a moderately to severely affected hand in executing a functional grasp independently, even in the presence of minimal voluntary finger flexion. This, in turn, may enable them to participate in task-oriented training interventions [17, 21, 27-29]. However, evidence on the effectiveness of the aforementioned approaches remains sparse.

As patients' motivation to train/exercise is correlated to therapy outcome [30], next to studying the effectiveness of a dynamic orthosis combined with electrical stimulation, it is important to investigate whether patients are motivated to work with this combination embedded in a task-oriented training program. Applying multiple devices may demand extra efforts as to time to 1) calibrate the orthosis to fit the anthropometrics of the affected forearm, wrist and hand, and 2) fine-tune the electrical stimulation prior to the task-oriented intervention. Furthermore, results as to regaining AHF and AHSP dexterity may be uncertain for the patient. Therefore, user experience (i.e. usability), perceived competences and motivational aspects from the patient's perspective (like values and beliefs related to task-oriented training combined with the orthosis and electrical stimulation) should be investigated. 
The aim of the present study was:

a) to evaluate to what extent arm-hand skill performance may improve in patients with a moderately to severely affected arm-hand (UAT score 1 - 2) in the sub-acute stage after a stroke who use a dynamic hand orthosis in combination with electrical stimulation, adjunct to therapy-as-usual, and

b) to assess the clinical usability of a dynamic hand orthosis combined with electrical stimulation, adjunct to therapy-as-usual, in patients with a moderately to severely affected arm-hand (UAT score 1-2) in the sub-acute phase after a stroke.

The following research questions were posed:

1) In which order of magnitude does a six-week training with a dynamic hand orthosis combined with electrical stimulation, adjunct to therapy-as-usual, improve armhand skill performance in patients with a moderately to severely affected arm-hand (UAT score 1-2) in the sub-acute stage after a stroke?

2) To what extent is a dynamic hand orthosis combined with electrical stimulation, adjunct to therapy-as-usual, usable in stroke patients with a moderately to severely affected arm-hand (UAT score 1 or- 2) in the sub-acute phase after a stroke?

\section{Methods}

\section{Study design}

This study featured a multiple baseline single case experimental design (A-B-A' design) [31] involving multiple single cases. From each individual subject many sequential measurements were recorded. After a baseline (A) period, an experimental intervention (B) was applied. In the subsequent time phase ( $\left.A^{\prime}\right)$ the effect of this experimental stimulus or intervention relative to baseline values was investigated. The number of measurements during baseline was varied between 3 and 6 (range: $12-30$ days). Three measurements were performed during the intervention phase $(B)$ and five during the follow-up phase ( $\left.\mathrm{A}^{\prime}\right)$. Measurements performed in the intervention and follow-up phase were interspaced by two weeks, thus generating a time series per 
measure for each patient separately (see also Figure 1). A meta-analysis on the pooled single case data were also performed.

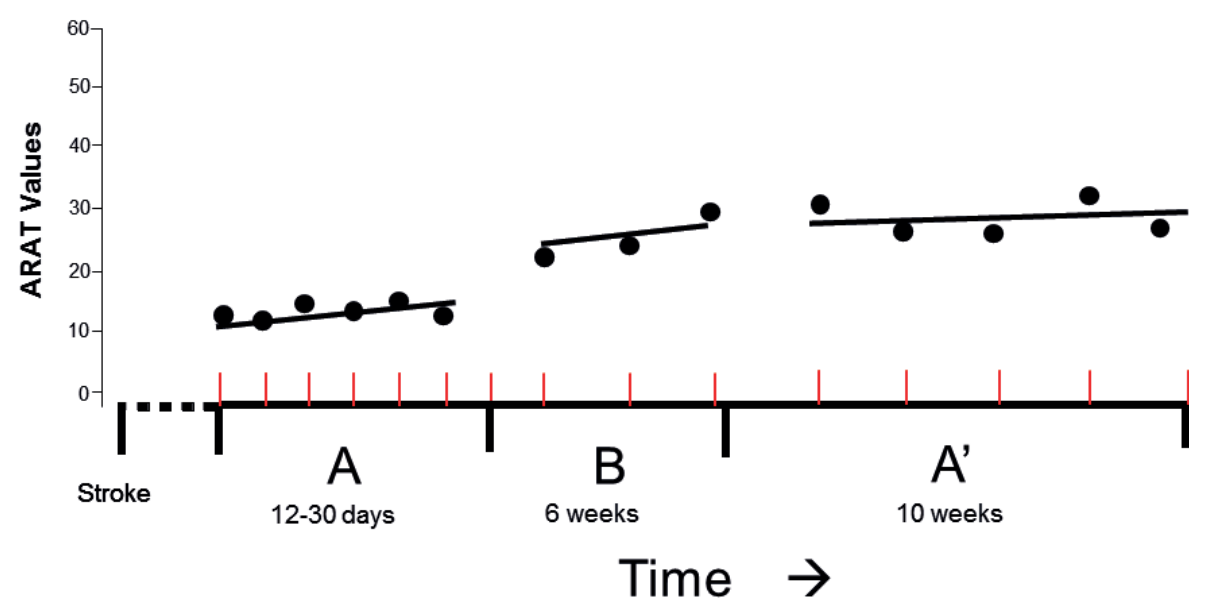

Figure 1. Example of linear detrending of the Action Research Arm Test time series of 1 subject. ARAT: Action Research Arm Test; BL: Baseline; TR: Training; FU: Follow- up.

\section{Subjects}

The study population consisted of eight sub-acute, first-ever stroke patients. All patients were clinically diagnosed with a moderately to severely impaired arm-hand, according to the Utrecht Arm-hand Test (UAT) [9], with an UAT score of 1 or 2. All patients were admitted to the inpatient stroke ward of Adelante Rehabilitation Centre in Hoensbroek, The Netherlands. Additional inclusion criteria were: age >= 18 years; patients' post-stroke time less than three months; a fair cognitive level, i.e. being able to understand the questionnaires and measurement instructions; ability to control sitting posture; optimal passive wrist extension of $>5^{\circ}$ (with a minimum of $-5^{\circ}$ ) as measured with the metacarpophalangeal, proximal interphalangeal and distal interphalangeal joints in extension; the distal part of the affected arm-hand impeded due to hypotonic muscles. Exclusion criteria were: serious problems regarding vision, leading to limited possibilities in object location and object recognition during goaldirected reach and grasp performance; severe problems of the shoulder, arm or hand on the paretic side, like edema and/or inflammation in the shoulder joint which hamper the execution of voluntary movements and/or the intensity of training; intolerance 
regarding the application of electrical stimulation; insufficient understanding of the Dutch language, no informed consent.

Participants were asked for their cooperation, and, after having received adequate information about the study, were enrolled after having given their consent.

\section{Apparatus}

A dynamic hand orthosis (Saeboglove, Saebo Inc, Charlotte, NC) was used. It consists of two parts, 1) a soft Lycra glove and 2) a plastic spiraled forearm shell. The glove assists in finger and thumb extension via tensioners located at all interphalangeal joints of each finger. The plastic forearm shell positions the wrist in a neutral, i.e. functional, position. The hand is maintained in an optimal position while grasping. Releasing is facilitated through finger extension just after the grasp movement has ended. The support of hand opening can be adjusted by therapist or the patient, depending on the amount of assistance needed to accomplish tasks. As the patient progresses, the tensioners can be removed individually as needed. When properly fitted, patients should be able to grasp and release different objects sized approximately 2 inches.

An electrical stimulation device, the Microstim 2(v2) (Odstock Medical Limited, Salisbury, Wiltshire) was used additional to the hand orthosis. The electrical stimulation was applied by placing two surface electrodes sized $2 \times 2$ inch. The anode was placed in the forearm midline at approximately one third of the muscle belly length of the musculus flexor digitorum profundus measured from the belly's proximal end [32].

The cathode was positioned on the muscle belly $5 \mathrm{~cm}$ distally from the anode [33]. The stimulator parameters were adjusted to time phases of $10 \mathrm{sec}$ with $200 \mathrm{usec}$ pulses of $40 \mathrm{~Hz}$, with $10-20 \mathrm{sec}$ silent period between the stimulation epochs. The current intensity was modulated till a tangible contraction of both muscles was present and fingers were flexed in order to produce a grasp movement.

An overview of the training set-up is presented in Figure 2 


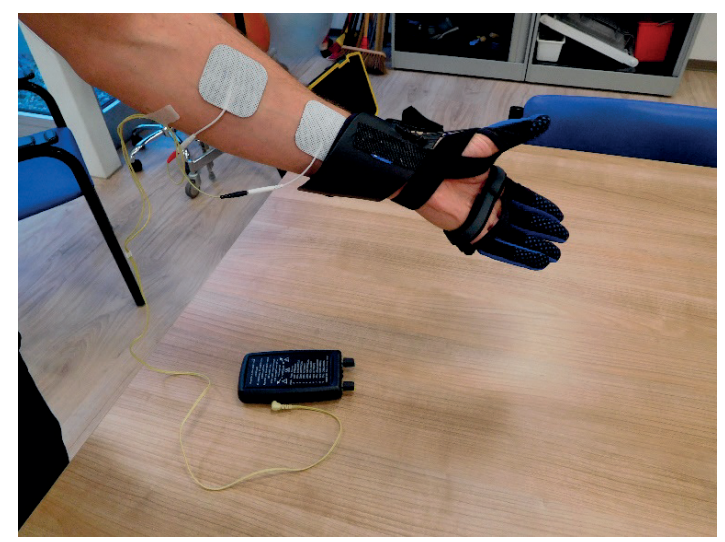

Figure 2. Overview of the treatment set-up.

\section{Intervention}

During the baseline assessment phase (A), patients received a protocolized arm-hand rehabilitation program (therapy-as-usual), i.e. program 1 of CARAS [34]. This program is designed to teach patients how to cope with a severely impaired, i.e. non-functional, arm-hand that cannot be used in daily activities. In this program patients learn strategies to avoid discomfort, to maintain joint mobility and to maintain their muscles/tendons in an optimal condition. Also, they learn strategies as to what to do when discomfort arises, and, during two times 30 minutes/week, they learn how to perform basic exercises to elicit voluntary movement where possible. Furthermore, patients learn how to maintain their affected arm-hand in an optimal condition and optimal position during different circumstances. Patients also receive training in the use of supportive tools like static or dynamic splints, braces and/or slings.

Prior to the intervention phase, the orthosis was fitted by a senior occupational therapist or a senior physical therapist. Concomitant with the orthosis, all patients received electrical stimulation. As soon as the patient experienced a comfortable fit of the orthosis, practice conditions were tested in a session, lasting 30 minutes, to observe if the patient was capable to perform various gross motor grip, grasp and release exercises independently. Once the patient succeeded, (s)he then entered the intervention phase. 
During the intervention phase $(\mathrm{B})$, patients followed a task-oriented training program, i.e. a gross motor grip performance program (program 2 of CARAS) [34] in conjunction with a dynamic hand orthosis (Saeboglove) and electrical stimulation. In this phase patients learned to integrate their affected arm in daily pursuits. Individual goal-setting and principles of self-efficacy were integrated to enable patients' maximum involvement during training. The duration of the training was 1.5 hours per day, 3 days per week for 6 weeks. Training sessions were tailored as follows: Patients started with training on a personal goal for 5 - 10 minutes, followed by 45 minutes of training with the dynamic orthosis, executing various grasp and release exercises. After these 45 minutes of training the patient worked $5-10$ minutes towards a personal goal again. Prior to the training sessions, a therapist facilitated the patient with setting up exercises fitting the patient's goals and current performance level.

During the final $\left(A^{\prime}\right)$ phase patients continued by following a second and final 6-week task- oriented training program, i.e. program 2 of CARAS) [34]. In this phase, neither the orthosis nor the electrical stimulation or any other supporting technology was used during arm-hand training.

No other, separate rehabilitation intervention involving the affected arm and hand was applied in the patients during this final phase.

\section{Measures}

\section{Utrecht Arm/Hand Test}

The Utrecht Arm-hand Test (UAT) is a simple bedside test measuring arm-hand motor impairment after stroke. Evaluation criteria are comparable to stages of motor recovery after stroke. The ordinal scale represents eight stages, ranging from 0 (non-functional arm) to 7 ('clumsy hand') [9]. In the present study the UAT was used as a classification measure at baseline.

\section{Action Research Arm Test}

The Action Research Arm Test (ARAT) is a capacity test [4] regarding upper extremity activity. It consists of four subtests comprising 16 grasp movements and three reaching 
movements to be performed by the patient. Items are scored on a 4-point scale, its sum score ranging from 0 to 57 . The test is valid [35], reliable [36] and sensitive to change [37] in patients with stroke. The ARAT is suitable for the evaluation of changes in arm-hand skills capacity in clinical trials [38]. The minimum clinically important differences (MCID) of the ARAT were 12 points (affected dominant arm-hand) and 17 points (affected non-dominant arm-hand [39].

\section{Intrinsic Motivation Inventory}

The Intrinsic Motivation Inventory (IMI) assesses participants' subjective experience related to a target activity. It has been used in several experiments related to intrinsic motivation and self-regulation $[40,41]$. In this study, 35 items extracted from the original 45 items of the IMI have been used (see appendix). The subscale 'Perceived choice' and 3 questions from the last subscale, i.e. 'Relatedness', have been excluded, since no single participant was able to elicit voluntary hand movements at the initial phase of this study and they all chose to participate in the intervention prior to the start of the study. The three questions removed from the last section, Relatedness, were aimed at interactions between persons, and therefore were less relevant to the research question. The 7-point Likert scale instrument was administered at the end of the follow-up phase to assess participants' interest/enjoyment, perceived competence, effort, value/usefulness, pressure/tension and relatedness felt while training with the dynamic spring-loaded hand orthosis. Please note that the item "pressure/tension felt" is scored inversely, relative to all other items of the IMI [40, 41]. The reliability of the IMI was found to be good [42].

\section{Data analysis and statistics}

The intervention (phase B) started at different time periods post-stroke due to the baseline lengths (phase $A$ ) differing between individual subjects (= multiple baseline design) [31].

This was done to methodologically ensure that the possible temporal causal relationship between the start of the intervention (phase $B$ ) and the occurrence of an effect of this intervention may become clear relative to any effects that may be 
observed during baseline (phase A) caused by e.g. spontaneous recovery and/or conventional therapy received.

In a first data analysis step, baseline data stability and any baseline trends regarding the ARAT, i.e. the average change between consecutive baseline measurement dates, were calculated per subject. Within-group differences between mean baseline data, mean training phase data and mean follow-up data were analysed using a Friedman two-way analysis of variance by ranks. Subsequent multiple comparison involved Wilcoxon signed ranks tests in a Bonferroni approach, thus correcting for spurious false positive findings.

Secondly, by using a least squares method, all ARAT data in the time series were linearly adjusted for baseline trends per subject to compensate for improvements caused by e.g. spontaneous recovery and/or conventional therapy received. The residuals, i.e. the detrended (and thereby rendered mutually independent) data, were subsequently analysed for the whole group using a Kruskal-Wallis one-way analysis of variance test, and, where applicable, followed by multiple comparison involving Mann-Whitney U-test.

Thirdly, the linear detrended ARAT time series (i.e. series of residuals) of each individual patient was analysed separately, i.e. per subject, the residual baseline measurement results were compared to the residual follow-up measurement results using Mann-Whitney U-tests.

Patients' experience and motivation are reported descriptively.

Linear detrending of the ARAT data was performed using MATLAB software (The MathWorks Inc, Natick, MA). All data were statistically analysed using IBM SPSS software version 23 (IBM Inc, Houston, TX). 


\section{Results}

Error analysis

Eight patients participated in this study. No data were missed during data acquisition. No adverse events were reported during the study.

\section{Participants' characteristics}

All patients were in the early sub-acute phase after stroke, i.e. between 24 hours till 3 months post-stroke. Patients' characteristics at entry in the study are presented in Table I.

Baseline data stability

Three (P3, P7 and P8) of the eight patients showed small improvements (i.e. one point) on the UAT during the baseline phase, whereas only one patient (P5) improved on the ARAT (by 6 points) during the baseline phase. Further improvements at baseline level were not present.

\section{ARAT results}

\section{General improvement over time}

Boxplots of ARAT mean results are presented in Figure 3A. Overall, patients improved over time regarding the ARAT $(p=0.001)$. Furthermore, significant improvements were found between baseline phase (BL) and follow-up phase (FU) $(p=0.012)$, baseline phase and training phase $(T R)(p=0.012)$, and between TR and FU $(p=0.017)$. 


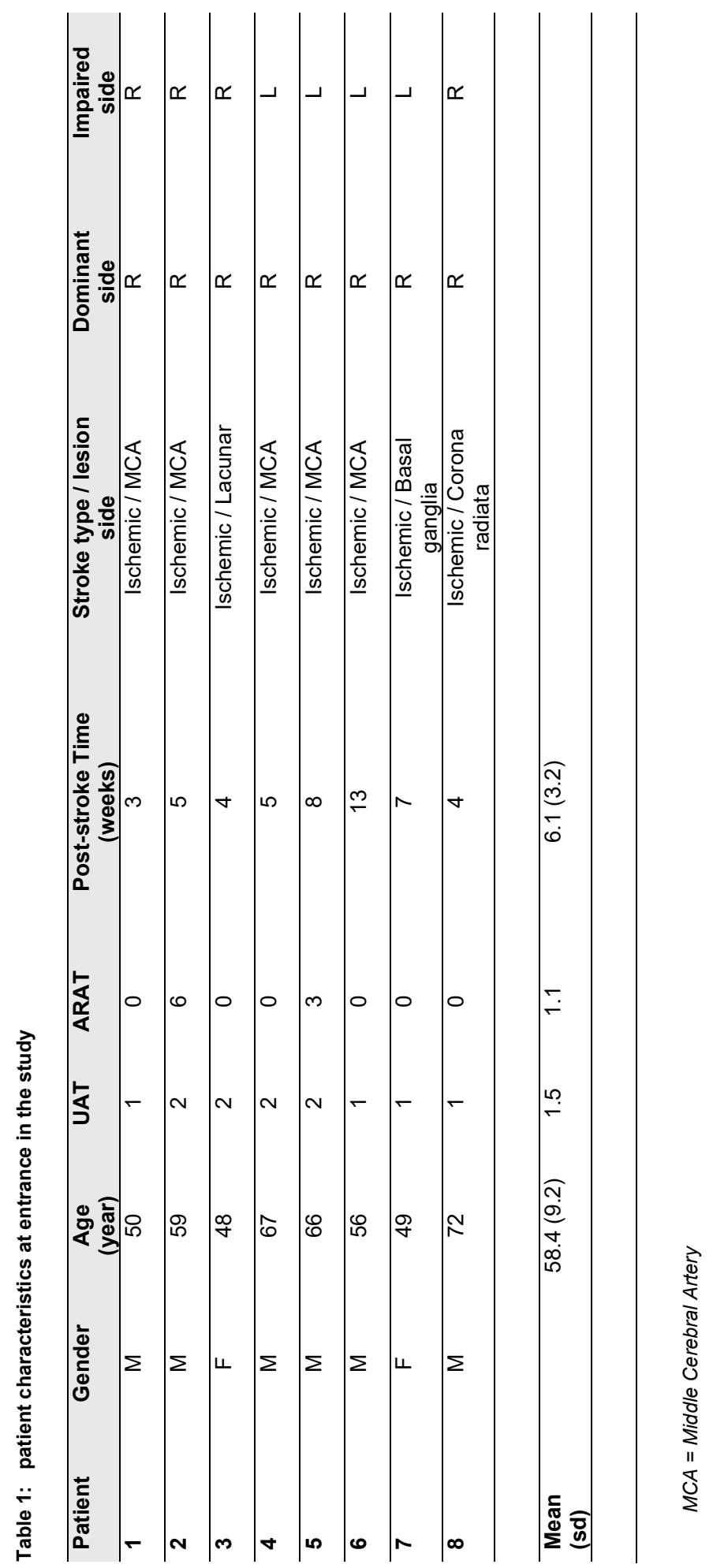




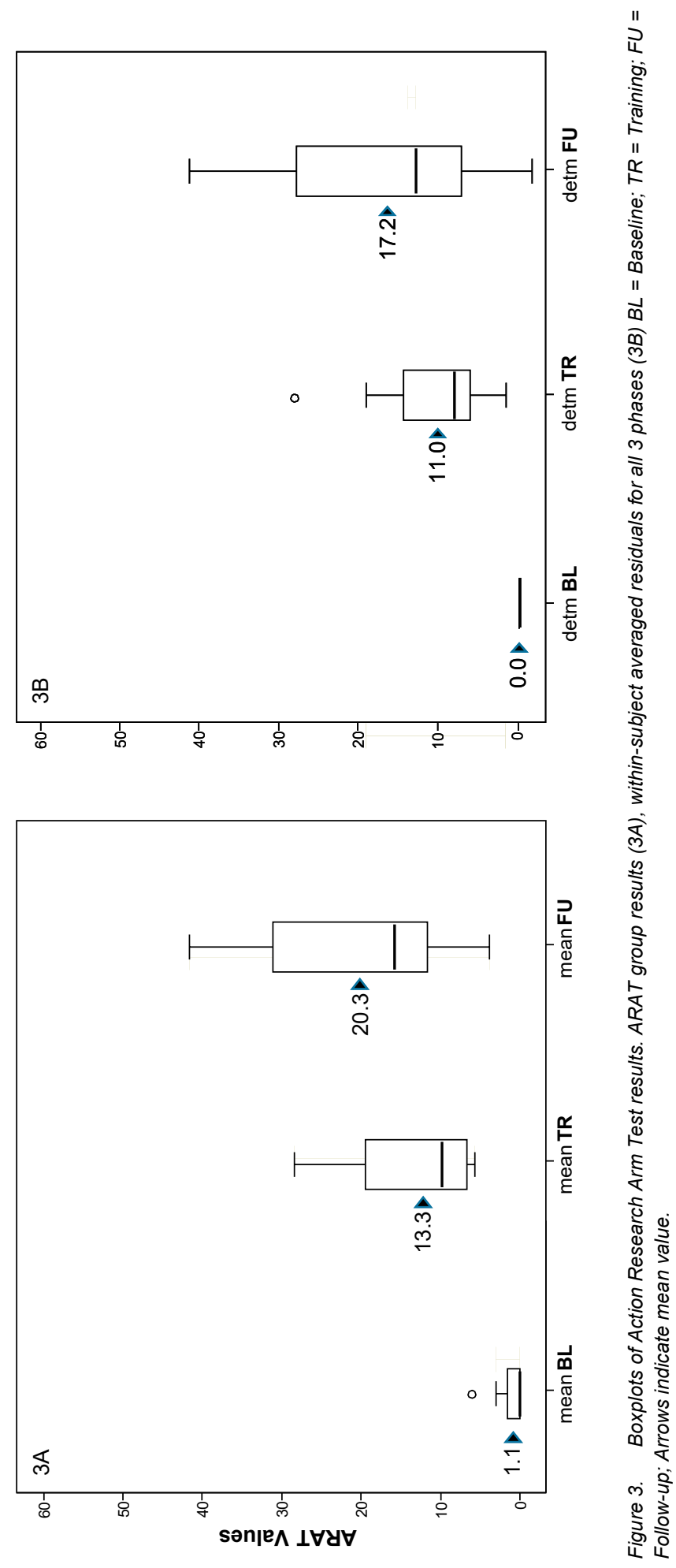


Improvement over time, corrected for baseline trends

Representing data of the whole group $(n=8)$, boxplots of the within-subject averaged ARAT time series data for all three phases, i.e. baseline, training and follow-up phase, linearly detrended for the baseline trends, are presented in Figure 3B.

An overall improvement was found for the ARAT results $(p=0.002)$. Multiple comparisons revealed that ARAT residuals were higher in FU relative to those obtained from BL $(p=0.010)$ and higher in TR relative to those obtained from $B L(p<0.000)$. No statistical differences in ARAT data were found between TR and FU $(p=0.293)$.

Time series for individual participants

In Figure 4 the baseline trend-corrected time series concerning the ARAT data per patient are depicted.

Furthermore, boxplots of ARAT time series residuals for the baseline (BL), training phase (TR) and follow-up phase (FU) are presented in Figure 5A and 5B.

In six of the eight patients (P1- P4, P6 - P7) mean (and median) ARAT residuals were higher $(p<=0.010)$ in the FU relative to the BL. One patient (P8) also improved in the FU relative to the $\mathrm{BL}$, but this improvement did not attain the level of statistical significance $(p=0.020)$. In one patient (P5) a decrease, though not statistically significant, in mean (and median) ARAT residuals was observed between the baseline phase and follow-up phase $(p=0.251)$. 

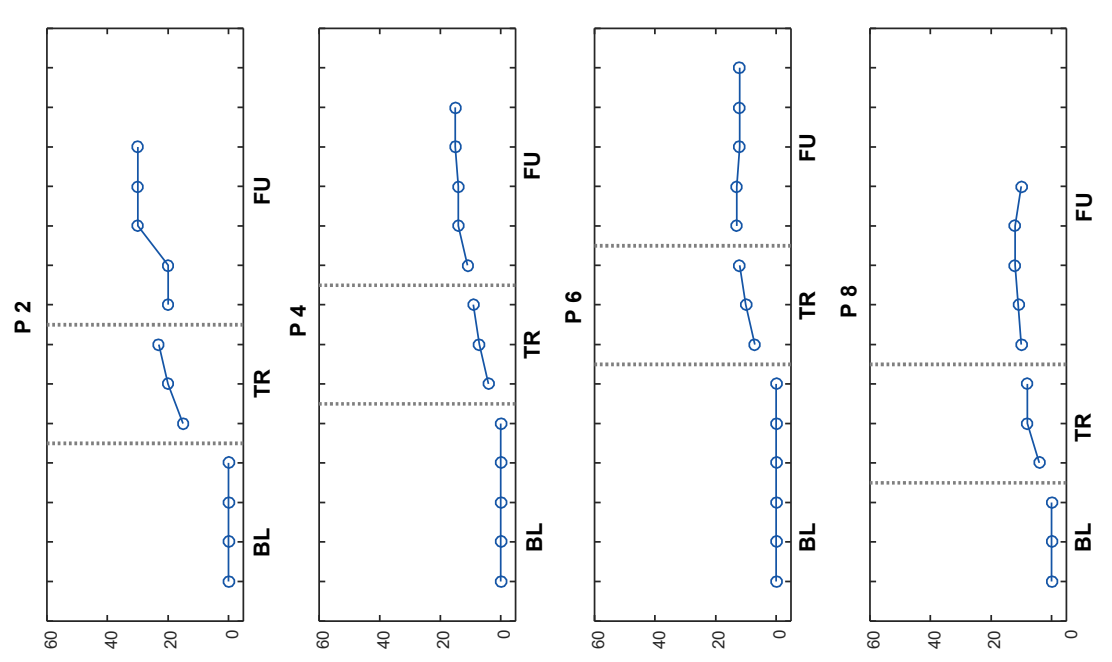

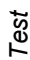

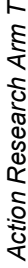

8

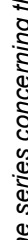

है

ษ

ํํㅇ

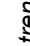

$\stackrel{Ð}{\$}$

$\infty$

$\frac{\pi}{2}$

के

हा

인

원

कू
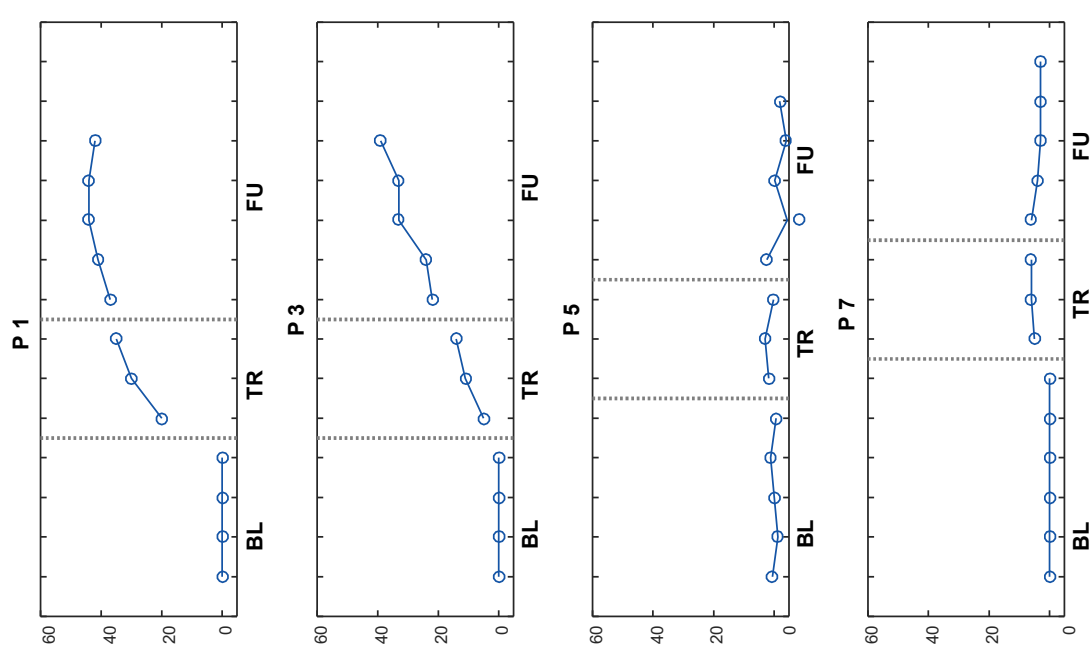

ธิ

:

足

के ब

일

ठิ

पे ले

\&

है ?

ऽ

就

बิ

워

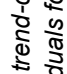

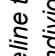

胥

еұер рәриәдәр $\perp \forall y \forall$

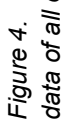




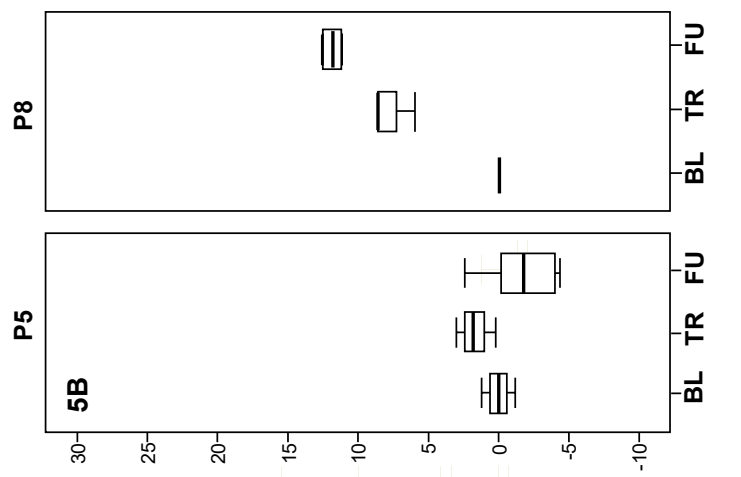

政
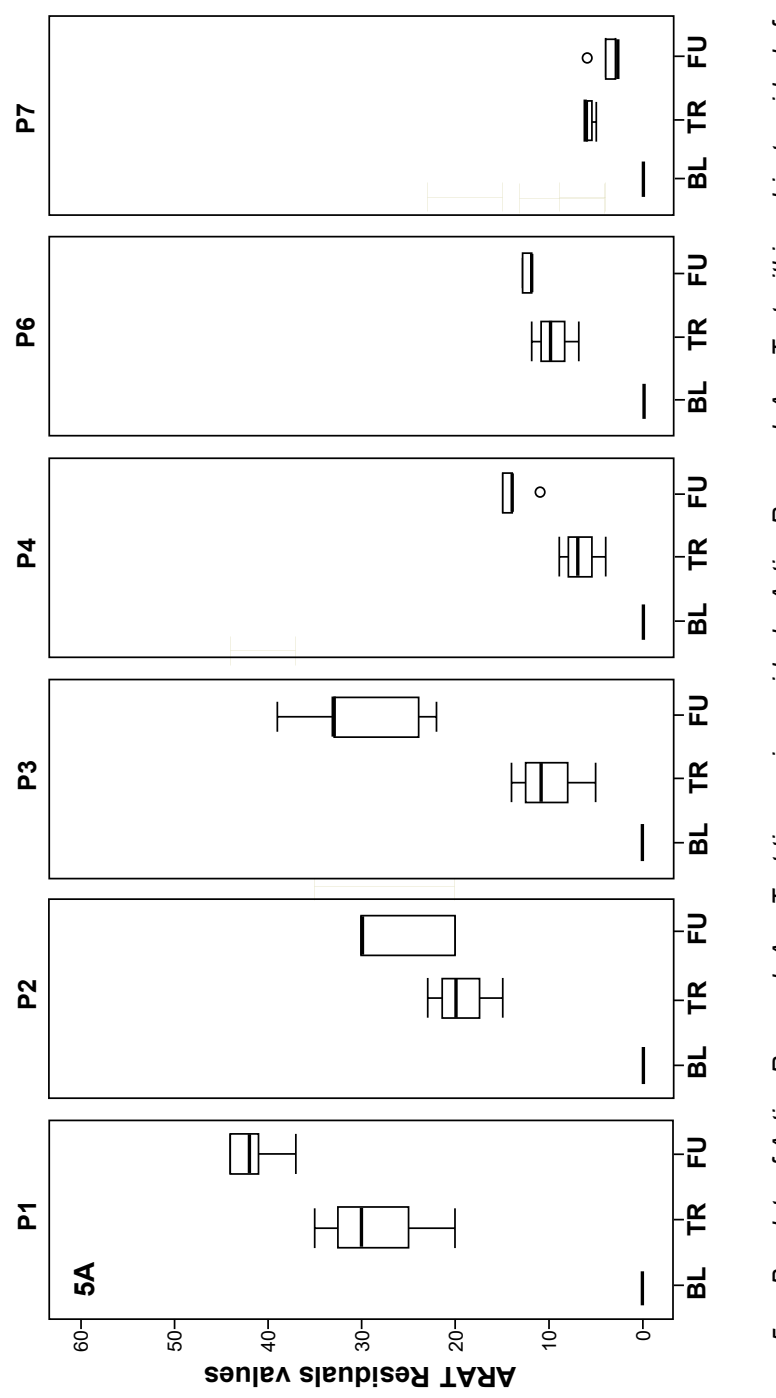
Six of the eight patients (P1 - P4, P6 - P7) also showed a statistically significant improvement between $B L$ and TR ( $p<=0.019$ ). One patient (P8) improved over time, although this improvement did not attain significance level $(p=0.034)$. One patient (P5) did not improve between BL and TR $(p=0.101)$. Between the TR and FU phase no statistically significant improvements in ARAT residuals were observed.

With respect to the non-dominant and dominant hand, minimal clinically important difference (MCID) values differ. Three out of the eight patients who participated in this study exceeded the MCID threshold regarding the ARAT scores.

In Table 2 mean IMI results are displayed.

Intrinsic motivation and self-regulation values, as measured by the IMI, were relatively high for 3 of the 6 the IMI' sub items, i.e. 'value/usefulness' (6.3 / 7.0), 'effort/importance' (5.1 / 7.0) and 'relatedness' (4.9 / 7.0). The sub-item 'Experienced Pressure and Tension' was scored relatively low $(3.3 / 7.0)$.

Table 2: Overview of Intrinsic Motivation Inventory scores

\begin{tabular}{llc}
\hline Intrinisc Motivation Inventory Item & Mean & (sd) \\
\hline Interest / Enjoyment & & \\
\hline Perceived competence & 4.6 & $(0.9)$ \\
\hline Effort / Importance & 4.6 & $(1.1)$ \\
\hline Pressure / Tension & 5.1 & $(0.5)$ \\
\hline Value / Usefulness & 3.3 & $(0.5)$ \\
\hline Relatedness & 6.3 & $(0.9)$ \\
\hline
\end{tabular}




\section{Discussion}

The aim of this study was A) to evaluate in which order of magnitude arm-hand skill performance (AHSP) may improve in sub-acute stroke patients with an moderately to severely affected arm-hand (UAT score of 1 or 2), who use a dynamic hand orthosis in combination with electrical stimulation within a six-week task-oriented training program, and B) to assess the clinical usability of the dynamic hand orthosis combined with electrical stimulation, adjunct to therapy-as-usual, in patients with a moderately to severely affected arm-hand in the sub-acute phase after stroke.

As a result of the arm-hand training featuring the dynamic hand orthosis in combination with electrical stimulation, $75 \%$ of the sub-acute stroke patients improved on their capacity to use their affected arm in daily activities between the start of the training and follow-up. However, as all eight participants were included during the sub-acute phase after stroke, the improvements in arm-hand skill performance may also be attributable to other factors, i.e. a) spontaneous recovery, b) received therapy-as-usual, c) the application of the dynamic orthosis in combination with electrical stimulation or d) a combination of these factors.

In order to reduce the influence of spontaneous recovery and possible therapy-asusual effects on the data obtained, two methodological approaches were used. First, a 'multiple baseline across subjects' design was used. Secondly, data series were detrended for any baseline trend for each subject in order to identify the unique contribution of the applied intervention.

As to differences between baseline and follow-up phase, the trend-corrected ARAT data (or 'ARAT residuals') showed that, at group level, significant improvement in armhand skills capacity occurred. This suggests that patients with a moderately to severely impaired hand function at the initial start of the intervention can regain a certain level of dexterity at follow-up that may be attributed to the use of the dynamic orthosis in combination with electrical stimulation embedded in a task-oriented training program.

As to each individual patient's baseline detrended time series, six out of the eight patients demonstrated significant improvements in arm-hand skills capacity, indicating 
that they had benefitted from the orthosis in combination with electrical stimulation. In three out of these six patients arm-hand skills capacity improved to such an extent that it enabled them to routinely perform grasp and displace tasks as well as bimanual activities. The other three patients improved to a level which enabled them to perform gross motor grip function, i.e. being able to use their affected arm-hand for passive and active stabilization tasks like fixating bread while making a sandwich. However, one patient did not benefit from the orthosis in combination with the electrical stimulation at all. During the first two weeks of training this patient experienced an increase in muscle tone and spasticity in the forearm and finger flexors, hampering him in the task-oriented training. Finally, one patient showed clinically relevant, though statistically nonsignificant improvements in arm-hand skill capacity. The overall results of the present study are in line with Barry et al. [43] and Franck et al. [24] who found that stroke survivors with a moderately to severely affected arm-hand improved significantly on the ARAT after they received arm-hand training in conjunction with a dynamic orthosis.

The present study showed that, at group level, improvements in arm-hand skills capacity between the training phase and follow-up phase were considerable, though not statistically significant. The latter was mainly due to large inter-individual differences as gauged by the ARAT. From our finding, one might conclude that the majority of patients who improved during the training phase were able to at least maintain their arm-hand skill capacity level during the follow-up phase. Some of these patients even improved further as to arm-hand capacity during the follow-up phase.

In general, patients were motivated to work with the orthosis and electrical stimulation within the task-oriented training program that lasted 6 weeks. The relative high score regarding 'value' and 'usefulness' may indicate that patients were capable to work actively and independently with their affected arm and hand during training sessions. Most patients became more competent in the orthosis fitting procedure and (re)adjustments of the orthosis, leading to the device being more useful to them. The patients' growing belief in their own capabilities to become more skilled in the orthosis' fitting procedure and the use of the orthosis within arm-hand therapy may have positively influenced their general sense of self-efficacy [44]. This, in turn, may stimulate each patient to engage even more in his own rehabilitation program and in 
the use of his affected arm in daily pursuits, thus also preventing 'learned non-use' once he has returned to his home environment.

In this study, the application of the Saeboglove dynamic hand orthosis was combined with electrical stimulation and task-oriented training (CARAS) [34], in which patients were encouraged to work as independently as possible. Studies into the effectiveness of assistive arm-hand devices applied in arm-hand rehabilitation are upcoming [18, 23, $45,46]$.

The majority of these orthoses are actuated devices tested in acute, sub-acute and chronic stroke patients, but mainly in research conditions [18, 45]. The randomized controlled trial of Kutner et al. indicated that robot-assisted rehabilitation combined with task - oriented training enhances distal motor function of the wrist and fingers [28]. However, study participants had some voluntary hand movements, i.e. $\geq 10$ degrees of wrist/finger extension. The Rehand [47] is a dynamic hand orthosis which can be used easily during arm-hand skill performance tasks within rehabilitation practice. Due to its actuated finger-flexion and extension movements it may assist stroke survivors with no discernible voluntary motion in using their affected hand. However, as a grasp and release motion is generated by forearm and (intrinsic) hand muscle groups [47], some cortical connectivity between these muscles and the affected side of the brain should be present.

In stroke survivors with a severely affected arm-hand the application of electrical stimulation to improve arm-hand function has been proven to be non-beneficial [48]. In a case-report, Chin et al. combined functional electrical stimulation with a neuroprosthesis to restore AHF and AHSP in a severely affected stroke patient. Clinical relevant changes were observed at arm function level, but not at the level of dexterity of the severely affected hand [49].

In the present study the use of a non-actuated orthotic device in combination with electrical stimulation was found to be a valuable addition in arm-hand rehabilitation in 6 out of 8 patients with a severely affected hand in the sub-acute phase post stroke. This is in line with a study by Popovic et al. who combined orthotic systems with electrical stimulation to assist movements coupled to task-oriented training, and with Carpaneto et al $[20,50]$. 
Somatosensory dysfunction, expressed as lack of voluntary finger movements, prevents arm-hand recovery [51]. Despite upcoming clinically useful prediction models $[6,7,14,52]$ it remains difficult to reliably characterize those patients with a severe arm disability who will achieve a clinically important change during arm-hand training and those who will not $[53,54]$. Systematic monitoring during the first 8 to 12 weeks post-stroke onset has been advocated to identify subgroups of patients who might achieve dexterity in this limited time-window $[51,55]$.

The average time that the eight patients in our study entered the training phase was 7.6 weeks post-stroke onset. Prior to this intervention, all patients coped with a moderately to severely affected arm-hand, i.e. they did not show any arm-hand dexterity. Only voluntary movements in flexion synergy in the proximal and/or distal part of the arm (UAT 1-2) were observed. Once patients left the training phase, $75 \%$ of these patients had regained and maintained a certain level of arm-hand dexterity.

The present study shows that combining the orthosis with electrical stimulation allows patients to shift from a training condition in which they learn how to cope with a nonfunctional hand, i.e. program 1 of CARAS [34], towards participating within a taskoriented environment, i.e. program 2 of CARAS. This combination of therapy interventions may be a solution for patients with a moderately to severely affected armhand who otherwise might not recover functionally, as described in literature [51]. Where normally these patients had to cope with an uncertain prognosis as to attaining a certain level of arm-hand capacity or dexterity, we now should work on the early identification of the subgroup of patients that may have the potential to regain armhand dexterity within the sub-acute phase post-stroke, who might benefit from the therapy approach investigated in the present study. This, in turn, may lead to innovative insights to develop novel therapy methods or to adjust and combine existing therapy methods. 


\section{Considerations and future research}

Measuring changes in arm-hand skill performance of moderately and severely impaired stroke patients is important. However, it would also have been interesting to investigate how these results may be associated with outcome at the 'body and structure level' and the 'participation' level of the International Classification of Functioning (ICF) [56]. Another consideration is that generalizability of the results from a single case experimental design, as used in the present study, towards larger groups of patients, is limited.

The fact that one of the eight patients, i.e. patient P8, showed clinically relevant progressions that did not attain a statistical significance level, may possibly have been due to a lower number of baseline measurements (i.e. $n=3$ ) in this individual.In future research in this domain, it is advocated that baseline length should include at least four measurement points.

Future research should focus on: 1) optimizing arm-hand recovery models in order to assist therapists in identifying, at an early stage post-stroke, which patients might benefit from the application of assistive devices within arm-hand rehabilitation, and 2) larger scale clinical research (e.g. randomized clinical trials) into the effectiveness of assistive devices in stroke survivors in the sub-acute phase with a moderately to severely impaired arm-hand associated with loss of dexterity.

\section{Implications for rehabilitation}

- Arm-hand training featuring the dynamic hand orthosis in combination with electrical stimulation shows a shift from no dexterity to dexterity.

- As to the users' experience regarding the dynamic hand orthosis, patients perceive a high intrinsic motivation and sense of self-regulation.

- Combining the orthosis with electrical stimulation creates opportunities for a nonfunctional hand towards task-oriented training. 
CHAPTER 7

\section{Acknowledgements}

We are grateful to all patients who participated in this study and to all therapists who contributed to this study.

\section{Declaration of interest}

We have the following interests: JAF and HAMS are employed by Adelante. There are no patents, products in development or marketed products to declare. 


\section{References}

1. Broeks JG, Lankhorst GJ, Rumping K, Prevo AJ. The long-term outcome of arm function after stroke: results of a follow-up study. Disability and rehabilitation. 1999;21(8):357-64. PubMed PMID: 10503976.

2. Shelton FN, Reding MJ. Effect of lesion location on upper limb motor recovery after stroke. Stroke; a journal of cerebral circulation. 2001;32(1):107-12. PubMed PMID: 11136923.

3. World_Health_Organization. International Classification of Functioning, Disability and Health: ICF. Geneva: World Health Organization, 2001.

4. Lemmens RJ, Timmermans AA, Janssen-Potten YJ, Smeets RJ, Seelen HA. Valid and reliable instruments for arm-hand assessment at ICF activity level in persons with hemiplegia: a systematic review. BMC Neurol. 2012;12:21. Epub 2012/04/14. doi: 10.1186/1471-2377-12-21. PubMed PMID: 22498041; PubMed Central PMCID: PMCPMC3352056.

5. Hayward K, Barker R, Brauer S. Interventions to promote upper limb recovery in stroke survivors with severe paresis: a systematic review. Disability and rehabilitation. 2010;32(24):1973-86. PubMed PMID: 20964563.

6. Nijland RH, van Wegen EE, Harmeling-van der Wel BC, Kwakkel G. Presence of finger extension and shoulder abduction within 72 hours after stroke predicts functional recovery: early prediction of functional outcome after stroke: the EPOS cohort study. Stroke; a journal of cerebral circulation. 2010;41(4):745-50. Epub 2010/02/20. doi: 10.1161/strokeaha.109.572065. PubMed PMID: 20167916.

7. Duncan PW, Goldstein LB, Horner RD, Landsman PB, Samsa GP, Matchar DB. Similar motor recovery of upper and lower extremities after stroke. Stroke; a journal of cerebral circulation. 1994;25(6):1181-8. Epub 1994/06/01. PubMed PMID: 8202977.

8. Roh J, Rymer WZ, Beer RF. Evidence for altered upper extremity muscle synergies in chronic stroke survivors with mild and moderate impairment. Frontiers in human neuroscience. 2015;9:6. Epub 2015/02/27. doi: 10.3389/fnhum.2015.00006. PubMed PMID: 25717296; PubMed Central PMCID: PMCPMC4324145.

9. Kruitwagen-van Reenen ET, Post MW, Mulder-Bouwens K, Visser-Meily JM. A simple bedside test for upper extremity impairment after stroke: validation of the Utrecht Arm/Hand Test. Disability and rehabilitation. 2009;31(16):1338-43.

10. Canning CG, Ada L, O'Dwyer NJ. Abnormal muscle activation characteristics associated with loss of dexterity after stroke. Journal of the neurological sciences. 2000;176(1):4556. Epub 2000/06/24. PubMed PMID: 10865092. 
11. Taub E, Miller NE, Novack TA, Cook EW 3rd, Fleming WC, Nepomuceno CS, et al. Technique to improve chronic motor deficit after stroke. Archives of physical medicine and rehabilitation. 1993;74(4):347-54. Epub 1993/04/01. PubMed PMID: 8466415.

12. Veerbeek JM, van Wegen E, van Peppen R, van der Wees PJ, Hendriks E, Rietberg M, et al. What is the evidence for physical therapy poststroke? A systematic review and metaanalysis. Plos one. 2014;9(2):e87987. Epub 2014/02/08. doi: 10.1371/journal.pone.0087987. PubMed PMID: 24505342; PubMed Central PMCID: PMCPMC3913786.

13. Winters C, van Wegen E, Daffertshofer A, Kwakkel G. Generalizability of the Proportional Recovery Model for the Upper Extremity After an Ischemic Stroke. Neurorehabilitation and neural repair. 2015;29(7):614-22. Epub 2014/12/17. doi: 10.1177/1545968314562115. PubMed PMID: 25505223.

14. Prabhakaran S, Zarahn E, Riley C, Speizer A, Chong JY, Lazar RM, et al. Inter-individual variability in the capacity for motor recovery after ischemic stroke. Neurorehabilitation and neural repair. 2008;22(1):64-71. Epub 2007/08/10. doi: 10.1177/1545968307305302. PubMed PMID: 17687024.

15. Nakayama H, Jorgensen HS, Raaschou HO, Olsen TS. Recovery of upper extremity function in stroke patients: the Copenhagen Stroke Study. Archives of physical medicine and rehabilitation. 1994;75(4):394-8. Epub 1994/04/01. PubMed PMID: 8172497.

16. Lee YY, Lin KC, Cheng HJ, Wu CY, Hsieh YW, Chen CK. Effects of combining robotassisted therapy with neuromuscular electrical stimulation on motor impairment, motor and daily function, and quality of life in patients with chronic stroke: a double-blinded randomized controlled trial. Journal of neuroengineering and rehabilitation. 2015;12:96. Epub 2015/11/02. doi: 10.1186/s12984-015-0088-3. PubMed PMID: 26520398; PubMed Central PMCID: PMCPMC4628254.

17. Hughes AM, Burridge JH, Demain SH, Ellis-Hill C, Meagher C, Tedesco-Triccas L, et al. Translation of evidence-based Assistive Technologies into stroke rehabilitation: users' perceptions of the barriers and opportunities. BMC health services research. 2014;14:124. Epub 2014/03/14. doi: 10.1186/1472-6963-14-124. PubMed PMID: 24620739; PubMed Central PMCID: PMCPMC4007558.

18. Bos R, Haarman C, Stortelder T, Nizamis K, Herder J, Stienen A, et al. A structured overview of trends and technologies used in dynamic hand orthoses. Journal of neuroengineering and rehabilitation. 2016;13(62):2 - 25.

19. Mehrholz J, Platz T, Kugler J, Pohl M. Electromechanical and Robot-Assisted Arm Training for Improving Arm Function and Activities of Daily Living After Stroke. Stroke; a journal of cerebral circulation. 2009. PubMed PMID: 19286592. 
20. Carpaneto J, Micera S. Application of orthoses and neurostimulation in neurorehabilitation in: Oxford book of Neurorehabilitation. Christopher K, editor. First edition Oxford, United Kingdom: Oxford University Press; 2015. p. 263-73.

21. Mehrholz J, Pohl M, Platz T, Kugler J, Elsner B. Electromechanical and robot-assisted arm training for improving activities of daily living, arm function, and arm muscle strength after stroke. The Cochrane database of systematic reviews. 2015;11:CD006876. Epub 2015/11/13. doi: 10.1002/14651858.CD006876.pub4. PubMed PMID: 26559225.

22. Rymer W, Jayaraman A. Promises and challenges of neurorehabilitation technology. In: Christopher k, editor. Oxford Textbook of Neurorehabilitation. First edition Oxford: Oxford United Kingdom: Oxford University Press; 2015.

23. Maciejasz P, Eschweiler J, Gerlach-Hahn K, Jansen-Troy A, Leonhardt S. A survey on robotic devices for upper limb rehabilitation. Journal of neuroengineering and rehabilitation. 2014;11:3. Epub 2014/01/10. doi: 10.1186/1743-0003-11-3. PubMed PMID: 24401110; PubMed Central PMCID: PMCPMC4029785.

24. Franck JA, Timmermans AA, Seelen HAM. Effects of a dynamic hand orthosis for functional use of the impaired upper limb in sub-acute stroke patients: A multiple single case experimental design study. Technology and Disability. 2013;25:177-87.

25. Davenport S. Recovery with grasp? A report on a preliminary study investigating the short and medium term effects of the SaeboFlex (FTM System) on chronic post stroke patients with residual upper limb deficit. Synapse. 2005;(spring):12-5.

26. de Araujo RC, Rocha DN, Pitangui AC, Pinotti M. The influence of dynamic orthosis training on upper extremity function after stroke: a pilot study. Journal of healthcare engineering. 2014;5(1):55-66. Epub 2014/04/03. doi: 10.1260/2040-2295.5.1.55. PubMed PMID: 24691386.

27. Langhorne P, Coupar F, Pollock A. Motor recovery after stroke: a systematic review. Lancet Neurol. 2009;8:741-54.

28. Kutner NG, Zhang R, Butler AJ, Wolf SL, Alberts JL. Quality-of-life change associated with robotic-assisted therapy to improve hand motor function in patients with subacute stroke: a randomized clinical trial. Physical therapy. 2010;90(4):493-504. Epub 2010/02/27. doi: 10.2522/ptj.20090160. PubMed PMID: 20185616; PubMed Central PMCID: PMCPMC2848350.

29. Burridge $\mathrm{JH}$, Hughes AM. Potential for new technologies in clinical practice. Current opinion in neurology. 2010;23(6):671-7. PubMed PMID: 20962639.

30. Dixon G, Thornton EW, Young CA. Perceptions of self-efficacy and rehabilitation among neurologically disabled adults. Clinical rehabilitation. 2007;21:230-40.

31. Barlow D, Nock, MK, Hersen, M. Single case experimental designs: strategies for studying behavior change. Cambridge: Pearson Publishing; 2008. 
32. Yang F, Zhang X, Xie X, Yang S, Xu Y, Xie P. Intramuscular nerve distribution patterns of anterior forearm muscles in children: a guide for botulinum toxin injection. Am J Transl Res. 2016;15(8):5485-93.

33. Watson C, Lyell K, Jones R. Compound muscle action potentials. 2009. In: clinical neurophysiology [Internet]. Oxford: Oxford press. third. [327- 267].

34. Franck JA, Halfens JAM, Smeets RJEM, Seelen HAM. Concise Arm and hand Rehabilitation Approach in Stroke (CARAS): A practical and evidence-based framework for clinical rehabilitation management. The Open Journal of Occupational Therapy. 2015;3(4):Article 10. doi: 10.15453/2168-6408.1164.

35. Hsieh CL, Hsueh IP, Chiang FM, Lin PH. Inter-rater reliability and validity of the action research arm test in stroke patients. Age Ageing. 1998;27(2):107-13. PubMed PMID: 16296669.

36. Lyle RC. A performance test for assessment of upper limb function in physical rehabilitation treatment and research. Int J Rehabil Res. 1981;4(4):483-92. PubMed PMID: 7333761.

37. Weerdt de WJG, Harrison MA. Measuring recovery of arm-hand function in stroke patients: a comparison of the Brunnstrom-Fugl-Mayer test and the Action Research Arm test. Physiotherapy Canada. 1985;37:65-70.

38. van der Lee JH, Roorda LD, Beckerman H, Lankhorst GJ, Bouter LM. Improving the Action Research Arm test: a unidimensional hierarchical scale. Clinical rehabilitation. 2002;16(6):646-53. PubMed PMID: 12392340.

39. Lang CE, Edwards DF, Birkenmeier RL, Dromerick AW. Estimating minimal clinically important differences of upper-extremity measures early after stroke. Archives of physical medicine and rehabilitation. 2008;89(9):1693-700. PubMed PMID: 18760153.

40. Deci EL, Eghrari H, Patrick BC, Leone DR. Facilitating internalization: the selfdetermination theory perspective. J Pers. 1994;62(1):119-42. PubMed PMID: 8169757.

41. Johnson MJ, Loureiro RCV, Harwin WS. Collaborative tele-rehabilitation and robotmediated therapy for stroke rehabilitation at home or clinic. Intel Serv Robotics. 2008;1:109-21.

42. Tsigilis N, Theodosiou A. Temporal stability of the intrinsic motivation inventory. Perceptual and motor skills. 2003;97(1):271-80. Epub 2003/11/08. doi: 10.2466/pms.2003.97.1.271. PubMed PMID: 14604050.

43. Barry JG, Ross SA, Woehrle J. Therapy incorporating a dynamic wrist-hand orthosis versus manual assistance in chronic stroke: a pilot study. Journal of Neurologic Physical Therapy. 2012;36(1):17-24. doi: 10.1097/NPT.0b013e318246203e. 
44. Jones F, Mandy A, Partridge C. Changing self-efficacy in individuals following a first time stroke: preliminary study of a novel self-management intervention. Clinical rehabilitation. 2009;23:522-33.

45. Balasubramanian S, Klein J, Burdet E. Robot-assisted rehabilitation of hand function. Current opinion in neurology. 2010;23(6):661-70. Epub 2010/09/21. doi: 10.1097/WCO.0b013e32833e99a4. PubMed PMID: 20852421.

46. Masiero S, Poli P, Rosati G, Zanotto,D. Losa, M. Paolucci S, et al. The value of robotic systems in stroke rehabilitation. Expert Rev Med Devices. 2014:1-12.

47. Nycz CJ, Butzer Thomas, Lambercy O, Arata J, Fischer GS, Gassert R. Design and Characterization of a Lightweight and Fully Portable Remote Actuation System for Use. IEEE Robotics and Automation Letters. 2016;1(2):976-83.

48. Kwakkel G, Winters C, van Wegen EE, Nijland RH, van Kuijk AA, Visser-Meily A, et al. Effects of Unilateral Upper Limb Training in Two Distinct Prognostic Groups Early After Stroke: The EXPLICIT-Stroke Randomized Clinical Trial. Neurorehabilitation and neural repair. 2016. Epub 2016/01/10. doi: 10.1177/1545968315624784. PubMed PMID: 26747128.

49. Marquez-Chin CM, A. Popvic, M. EEG-Triggered Functional Electrical Stimulation Therapy for Restoring Upper Limb Function in Chronic Stroke with Severe Hemiplegia. Case Rep Neurol Med. 2016. doi: 10.1155/2016/9146213.

50. Popovic DB, Popovic MB. Hybrid assistive systems for rehabilitation: lessons learned from functional electrical therapy in hemiplegics. Conference proceedings : Annual International Conference of the IEEE Engineering in Medicine and Biology Society IEEE Engineering in Medicine and Biology Society Annual Conference. 2006;1:2146-9. Epub 2007/10/20. doi: 10.1109/iembs.2006.259550. PubMed PMID: 17946941.

51. Winters C, Kwakkel G, Nijland R, van Wegen E. When Does Return of Voluntary Finger Extension Occur Post-Stroke? A Prospective Cohort Study. PloS one. 2016;11(8):1-12. doi: 10.1371/journal.pone.0160528.

52. Kwakkel G, Kollen BJ, van der Grond J, Prevo AJ. Probability of regaining dexterity in the flaccid upper limb: impact of severity of paresis and time since onset in acute stroke. Stroke; a journal of cerebral circulation. 2003;34(9):2181-6. PubMed PMID: 12907818.

53. Hayward K, Kuys S, Barker R, Brauer S. Can stroke survivors with severe upper arm disability achieve a clinically important change in arm function during inpatient rehabilitation? A multicentre, prospective, observational study. NeuroRehabilitation. 2014;35(1):17-23. Epub 2014/07/06. doi: 10.3233/nre-141096. PubMed PMID: 24990005.

54. Kwah L, Herbert R. Prediction of Walking and Arm Recovery after Stroke:

A Critical Review. brain sciences. 2016;6(53):1-16. 
55. Duncan PW, Matchar D, Divine GW, Feussner, J. Measurement of motor recovery after stroke. Outcome assessment and sample size requirements. Stroke; a journal of cerebral circulation. 1992;23(8):1084 - 9.

56. WHO. International classification of functioning, disability and health. 2001. 


\section{Chapter}

\section{Effects of a dynamic hand orthosis for functional use}

of the impaired upper limb in sub-acute stroke patients:

A multiple single case experimental design study

Johan Anton Franck, Annick Antoinette Alfonsine Timmermans,

Henk Alexander Maria Seelen

(10.3233/TAD-130374) 


\section{Abstract}

Objective: To investigate the usability and the effects of a dynamic spring-loaded orthosis, adjunct to therapy-as-usual (TAU), on functional use of the impaired hand in moderately/severely impaired sub-acute stroke patients.

Design: single case experiment (A-B-A'-design).

Subjects: Eight sub-acute stroke patients.

Methods: The orthosis was used for six weeks, five days/week, 45\%/day, and adjunct to TAU. Outcome measures: Action Research Arm Test (ARAT), ABILHAND, Intrinsic Motivation Inventory (IMI).

Results: At group level, patients improved on ARAT $(p=0.001)$ and ABILHAND $(p=0.005)$. After detrending for baseline trends (caused by e.g. spontaneous recovery and/or TAU), such improvement was only found for ARAT $(p=0.009)$. At individual level, three patients whose baseline ARAT changed little (0-3 points), had improved at follow-up, and four remained constant regarding detrended ARAT results. In four patients mean detrended ABILHAND results were higher during follow-up relative to baseline ( $p<=0.036$ ). Average IMI sub-scores were between 5.4 and 6.6 (of 7 ), except for 'pressure/tension' (2.1).

Conclusion: Patients, who, in the early sub-acute phase after stroke, display only little/modest improvement on their capacity to perform activities or their perceived level of daily performance, seem to benefit most from training with a dynamic arm orthosis. Patients perceived a high intrinsic motivation and sense of self-regulation. 


\section{Introduction}

In the Netherlands, in 2010 the incidence of stroke was 33,862 [1]. Thirty to $66 \%$ of the stroke survivors suffer from a severe upper limb paresis, and face long-term impaired arm function [2, 3]. Only 5-20\% of stroke survivors regain arm-hand function in the first weeks post-stroke and are able to re-use the impaired hand in daily life activities $[4,5]$. These patients experience mild limitations in daily occupations and are able to control finger and wrist extension [6, 7]. Approximately $20 \%$ of the stroke survivors suffer from a moderate paresis [8]. These persons will never regain hand dominance. However, they have the potential to regain dexterity and may be able to use their arm in basic functional tasks.

An important focus of stroke rehabilitation is to improve arm-hand skill performance to maximize functional motor ability [9]. Well-known treatment approaches to improve arm-hand performance after stroke are constraint-induced movement therapy (CIMT) [10], mental practice [11, 12], task-oriented training [13], and technology-supported rehabilitation [14, 15]. Although these approaches have been proven successful, most approaches have been evaluated in patients who are able to execute functional graspand-release tasks with their impaired arm and hand without assistance, i.e. in patients with voluntary wrist and finger extension in the paretic arm and hand [16]. For patients who suffer from a moderate or severe paresis, it is often difficult to participate in taskoriented interventions, as they lack the capacity for voluntary movement in their paretic hand.

Technology-assisted training, featuring robotics, electrical stimulation, gaming devices and/or assistive orthoses, can be used in conjunction with training of everyday tasks $[15,17,18]$. Mehrholz et al. [19] indicated in a systematic review that motor strength of the paretic arm and motor function are more likely to improve when patients after stroke train with electromechanical devices and allow stroke patients to practice intensively by themselves $[15,20,21]$. These electromechanical training systems of arm and hand performance after stroke may be roughly divided into passive (stabilising the arm and hand), active systems (actuators moving the arm and/or hand) and interactive systems, the latter of which react to patients' inputs to provide an optimal assistance strategy [22]. Most of these systems are equipped with high-end electronic, mechanical features and software. However, elaborating on the (dis)advantages of these devices 
is beyond the scope of this article. The orthosis as has been tested in the present study is a passive, mechanical device.

In neurorehabilitation in general, and more specifically in (chronic) stroke patients, effects of treatment regimes featuring orthoses are not unequivocal. At the impairment level, minor effects of stretching on muscle spasticity [23-25], strength, range of movement and grip have been reported [26, 27]. Modest improvements at activity and participation level are reported in a study by Butler [27], who used a dynamic springloaded hand orthosis (Saeboflex, Saebo Inc, Charlotte, NC) in one patient suffering from a chronic stroke. Such orthoses aim to assist persons who are not able to make a functional grasp with their paretic arm and hand themselves. The creation of a functional grasp by means of spring-loading finger extension enables patients to execute task-oriented grasp and release exercises and practice intensively using repetitive movements. This dynamic orthosis can be used in goal-directed activities and lowers the threshold for patients to participate in a greater variety of evidencebased treatment programs like group therapy and CIMT. From the perspective of (therapy) efficiency and patient satisfaction, the use of such orthosis may be beneficial, because less individual therapy assistance is needed.

To date, most clinical studies reporting effects of dynamic spring-loaded hand orthoses have been performed in chronic stroke patients. So far, only Davenport et al. [28]reported results, at patients' capacity level, in sub-acute stroke patients. A clinical assumption is that the benefits of such an orthosis may be larger in sub-acute stroke patients, because learned non-use [29] and secondary complications like contractures may be prevented. However, this has not yet been investigated systematically.

Besides evaluation of the effects of the use of a dynamic spring-loaded hand orthosis, it is essential to investigate whether patients are motivated to train with the orthosis. The level of motivation is an important beneficial factor in pursuing goals and activities [30] and in maintaining a high degree of training practice compliance. Therefore, information about user experience (i.e. usability), perceived competences and motivational aspects (like values and beliefs related to functional arm-hand training combined with the orthosis from the patients' perspective) should be gathered.

The aims of the present study have been to investigate a) the effects of a dynamic spring-loaded orthosis, adjunct to therapy-as-usual, on the functional use of the 
impaired upper limb in moderately to severely impaired sub-acute stroke patients, and b) patients' motivation regarding the use of a dynamic orthosis. The following research questions were posed:

1) Does a six-week, upper limb-based rehabilitation regime, assisted with a dynamic spring-loaded hand orthosis, improve arm-hand capacity and perceived daily activity performance beyond effects of spontaneous recovery and therapy-as-usual in patients with moderate to severe upper extremity paresis in the sub-acute phase after stroke?

2) How do moderately to severely impaired stroke patients rate user experience with regard to a dynamic spring-loaded hand orthosis during a six-week arm-hand training regime?

\section{Methods}

\section{Study design}

This study featured a single case experimental design (A-B-A' design) [31], involving multiple single cases. During the baseline phase $(A)$, intervention phase $(B)$ and followup phase $\left(A^{\prime}\right)$, three, two and five measurements were performed respectively, each interspaced by approximately two weeks, thus producing a time series, per measure, for each patient. Meta-analyses, on the pooled single case data, have also been performed.

\section{Subjects}

The current investigation was a sub-study in a large longitudinal clinical project called AMUSE (Activity Monitoring of Upper extremity use in Stroke patients during and after rEhabilitation), clinimetrically quantifying progress in arm-hand use at the patients' level of function, activity and participation. Patients suffering from a first-ever stroke, who were admitted to Adelante Rehabilitation Centre in Hoensbroek, the Netherlands, were asked to participate. 
Additional inclusion criteria were:

1) post- stroke time less than three months;

2) problems during the performance of daily activities due to a moderately to severely impaired upper limb;

3) no severe cognitive or communication problems;

4) clinically diagnosed with a paresis of the arm and hand, i.e.: Active range of motion (AROM) shoulder: $15^{\circ}-20^{\circ}$ (elevation/abduction); AROM elbow: $15^{\circ}$ $20^{\circ}$ flexion; Active finger flexion: $1 / 4$ active interphalangeal flexion during passive wrist extension $>15^{\circ}$ starting with full extension of the fingers; Passive range of motion: optimal wrist extension of $35^{\circ}$ (with a minimum of $15^{\circ}$ ) with metacarpophalangeal, proximal interphalangeal, and distal interphalangeal joints in extension.

Exclusion criteria were:

1) serious problems regarding vision or hearing;

2) severe arm edema;

3) severe shoulder pain;

4) insufficient understanding of the Dutch language.

All procedures and protocols of the AMUSE project were approved by the Medical Ethics Committee of the Maastricht University Medical Centre in Maastricht, the Netherlands. Written informed consent was obtained from all subjects prior to their participation.

\section{Apparatus}

A dynamic spring-loaded hand orthosis (Saeboflex, Saebo Inc, Charlotte, NC), assisting wrist extension muscles, and counterbalancing excessive activity of the wrist and finger flexors, was used. This orthosis allows subjects to use active wrist flexion to grasp objects. The orthosis is designed to position a non-functional hand in a biomechanically more optimal position, thus facilitating grasp and release activities. Using a fixed wrist extension angle $\left(35^{\circ}\right)$ and a variably loadable 'finger and thumb spring-loaded system' the optimal position of the hand is maintained and finger extension is supported after the grasp movement has ended. The orthosis is fitted and checked by a certified therapist, one criterion being the patient's ability to grasp a 3.5 inch soft foam ball. 


\section{Intervention}

During the baseline phase, the intervention phase and the follow-up phase, stroke patients received a conventional protocolized rehabilitation program (therapy-asusual), enabling them to optimize their arm-hand use in daily activities. During the intervention phase, this protocolized rehabilitation program was used in conjunction with a dynamic spring-loaded hand orthosis (Saeboflex). Key elements of the program are: 1) the patient is the main stakeholder and acquires knowledge about his possibilities and limitations regarding upper limb training; 2) the optimal integration of the paretic upper limb in daily occupations to improve arm and hand function; 3) the patients are enabled to maintain optimal care for their arm and hand.

Before the start of the program, the patient's self-perceived problems related to selfcare, productivity and leisure are identified and inventoried. In dialogue with the occupational therapist, a minimum set of three of these problems are translated into meaningful and attainable performance goals (individual goal setting). These goals should be directly related to home-based daily activities. Given their functional possibilities, patients will then undergo a personalised arm-hand training regime, within a training group of stroke survivors with a moderately to severely impaired arm and hand. As all group members experience and observe similar impairments among the other group members, group-wise training is an excellent (motivational) training tool [32]. During the execution of group tasks and individual tasks, each patient learns how to integrate his paretic arm and hand during manual activities in daily life situations. Special attention is given to 'active fixation' tasks, 'gross motor grip and displacement' tasks and simple bimanual daily life activities.

In a first session, the dynamic spring-loaded orthosis is fitted by a certified therapist. Subsequently, the participant will undergo the arm-hand training regime while wearing the orthosis. Patients start with training on a personal goal for 5 minutes, followed by 45 minutes of training with the dynamic orthosis, picking up and moving 3.5 inch soft foam balls. Immediately after this session the participant works for 15 minutes towards personal goals again. This training regime is followed for six weeks, five days per week, divided in three days during in the rehabilitation setting and two days at home. Participants work with a personal log and a self-administered home exercise program (including the use of the orthosis) during the weekends. 
A therapist is present during training to help with pre-training adjustment like arranging exercises and matching materials or configuring the dynamic orthosis to the patient's performance level. Within the first week of the program, the patient will learn to work with the orthosis, how the orthosis can be fitted properly without professional assistance and how to manage some exercises during the weekend, according to the homework assignment scheme.

\section{Measures used}

\section{Utrecht Arm/Hand Test}

The Utrecht Arm/hand-Test (UAT) is a simple bedside test measuring arm-hand motor impairment after stroke. Evaluation criteria are comparable to stages of motor recovery after stroke. The ordinal scale represents eight stages, ranging from 0 (non-functional arm) to 7 ('clumsy hand') [33]. In the present study the UAT is used for classification at baseline.

\section{Action Research Arm Test}

The Action Research Arm Test (ARAT) is a capacity test [34] regarding upper extremity activity. It consists of four subtests comprising 16 grasp movements and three reaching movements to be performed by the patient. Items are scored on a 4-point scale, its sum score ranging from 0 to 57. The test is valid [35], reliable [36] and sensitive to change [37] in patients with stroke. The ARAT is suitable for the evaluation of armhand activity changes in clinical trials [38].

\section{ABILHAND}

The ABILHAND is a clinical assessment tool evaluating perceived everyday performance of the impaired hand related to real life tasks [34, 39] using a set of 23 bimanual activities [40].The test is administered as a semi-structured interview, using a 3-level ordinal rating scale: impossible (0), difficult (1), and easy (2) to perform. Activities not performed in the last three months are not scored. To compare inter- or intra-individual manual ability, the ordinal scores are converted to an interval scale and consequently to a linear measure of manual ability, based on a Rasch analysis [39]. The ABILHAND is valid, responsive and clinically useful [40]. 


\section{Intrinsic Motivation Inventory}

The Intrinsic Motivation Inventory (IMI) assesses participants' subjective experience related to a target activity. It has been used in several experiments related to intrinsic motivation and self-regulation [41, 42]. The 7-point Likert scale instrument was administered at the end of the follow-up phase to assess participants' interest/enjoyment, perceived competence, effort, value/usefulness, pressure/tension felt, and perceived choice while training with the dynamic spring-loaded hand orthosis [41]. Its reliability was found to be good [43].

\section{Data analysis}

Baseline data stability and any trends regarding the ARAT and ABILHAND, i.e. the average change between consecutive measurement dates, were calculated. Subsequently, three approaches were used to analyse the ARAT and ABILHAND data. Firstly, to ascertain whether patients improved over time irrespective of training, differences between mean baseline data, mean training phase data and mean followup data were analysed using a Friedman two-way analysis of variance by ranks. Multiple comparison involved Wilcoxon signed ranks tests. Secondly, data were linearly detrended for baseline trends per subject, using a least squares method, to (partially) compensate for improvements caused by e.g. spontaneous recovery and/or conventional therapy received. An example of linear detrending of the ARAT time series of one subject is shown in Figure 1. 


\section{CHAPTER 8}
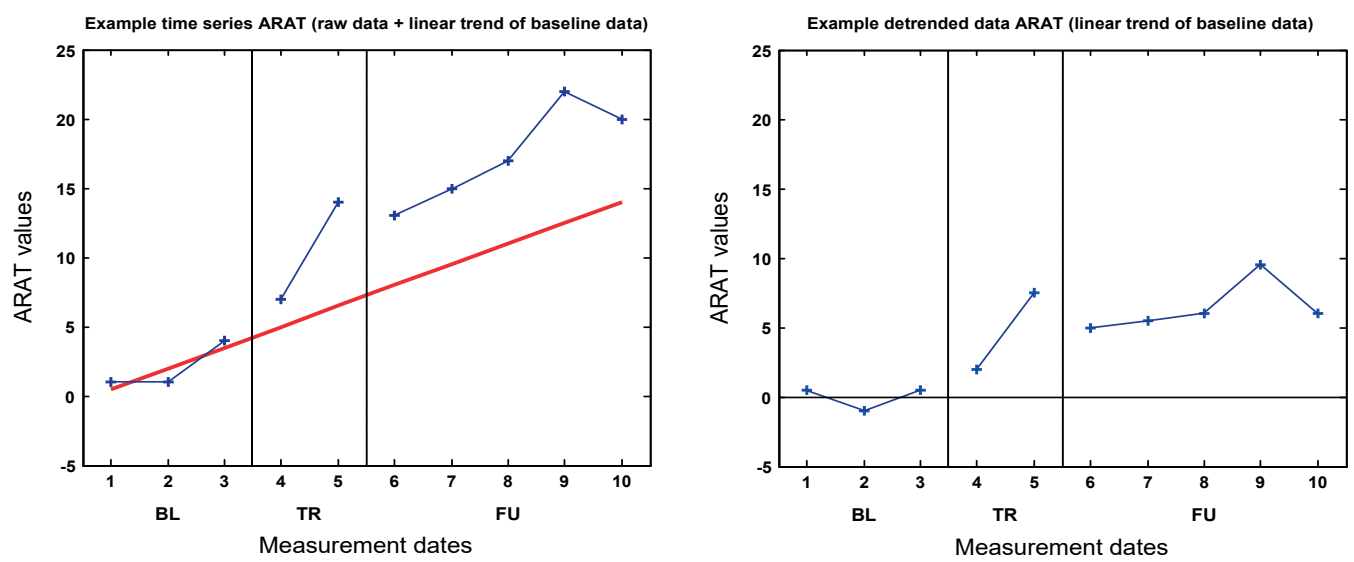

Figure 1. Example of linear detrending of the ARAT time series of 1 subject. ARAT = Action Research Arm Test; $B L=$ Baseline; $T R=$ Training; FU = Follow-up.

The residuals, i.e. the detrended (and thereby rendered mutually independent) data, were subsequently analysed for the whole group using a Kruskal-Wallis test, and, where applicable, followed by multiple comparison involving Mann-Whitney U-tests. Thirdly, the linear detrended time series (i.e. series of residuals) of each individual patient was analysed separately, i.e. per subject the residual baseline measurement results were compared to the residual follow-up measurement results using MannWhitney U-tests. The aforementioned procedures were applied to both the ARAT and ABILHAND data. Linear detrending of the data was performed using MATLAB software (The MathWorks Inc, Natick, MA). All data were statistically analysed using IBM SPSS software version 19 (IBM Inc, Houston, TX). Patients' experience is reported descriptively. 


\section{Results}

\section{Error analysis}

Ten patients entered the study. However, two patients (P8 and P10) withdrew from the study very early during the baseline measurement phase because of shoulder pain or autonomic dysregulation problems in the arm. No further data were missed during data acquisition. No adverse effects of the training were found. Patients' characteristics at entrance in the study are presented in Table 1.

\section{Baseline data stability}

In Table 2 baseline data as well as the average change, i.e. any improvement or deterioration, across the baseline phase that may have been due to spontaneous recovery and/or conventional therapy received, for the ARAT and the ABILHAND of each subject are presented.

Small improvements on the ARAT during baseline are foremost observed in P3, P7, $\mathrm{P} 1$ and $\mathrm{P} 4$. For the ABILHAND this primarily holds for P7, P3 and P9. Largest improvements during baseline were found in P2 and P6 (ARAT) and P2 (ABILHAND).

\section{ARAT results}

\section{General improvement over time}

Overall, patients improved over time on the ARAT $(p=0.001)$. Furthermore, ARAT group results were higher both during the training phase and during follow-up, relative to baseline data $(p=0.012)$. Boxplots of ARAT results are presented in Figure 2A. 


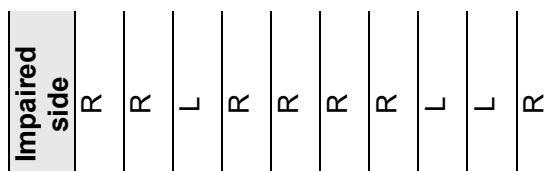

蒙

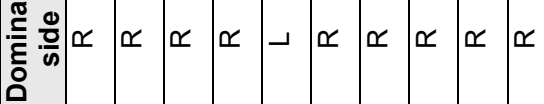

일

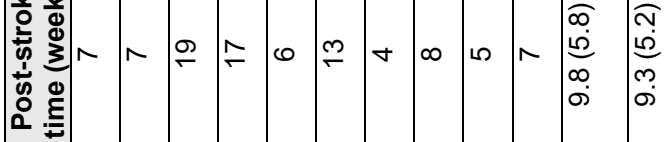

2.

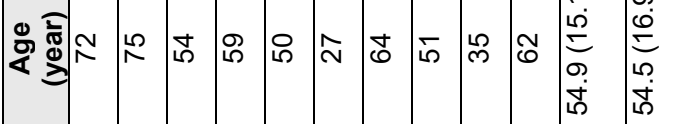

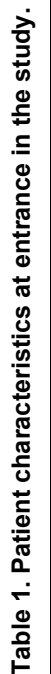

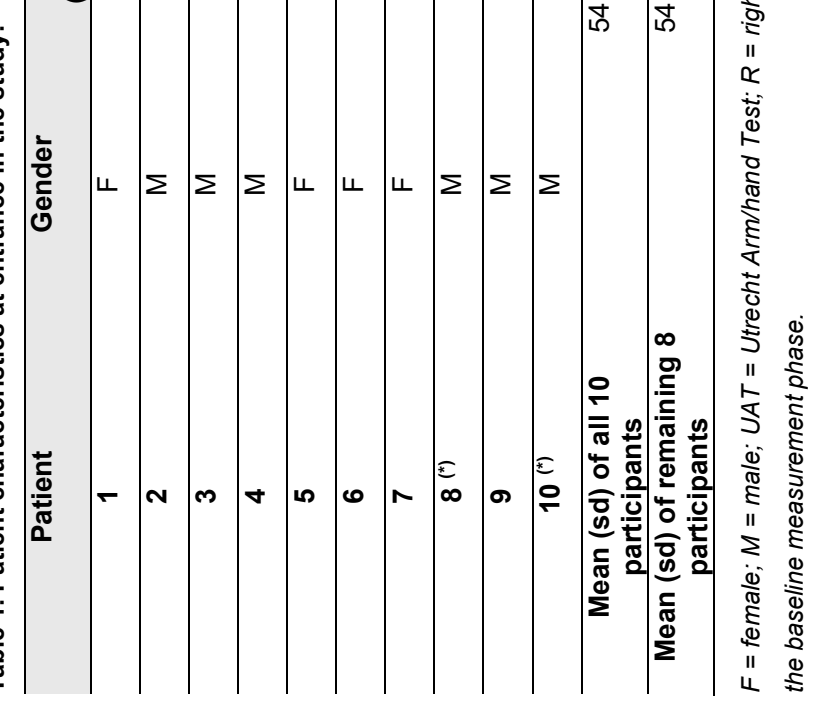




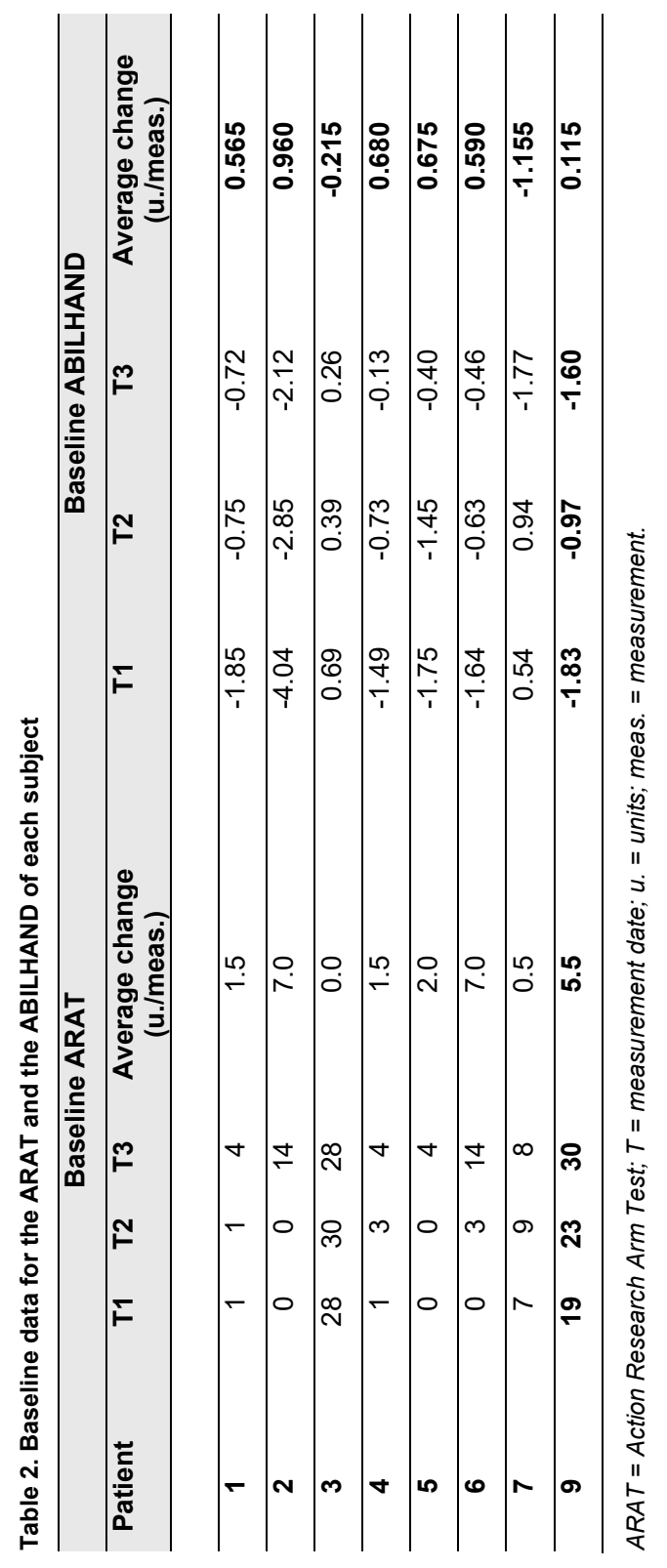


2A

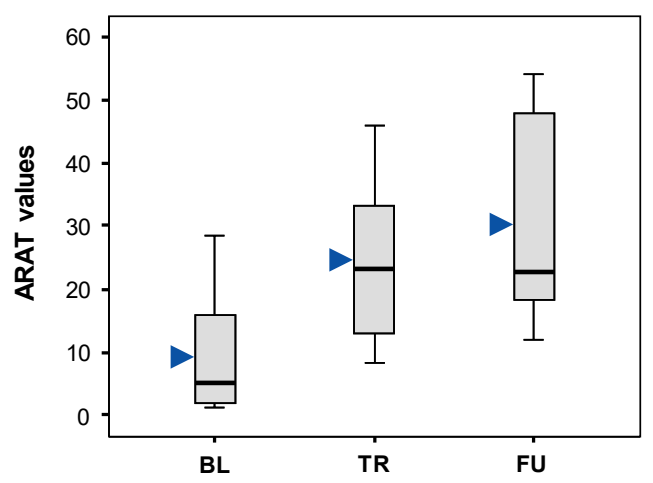

2B

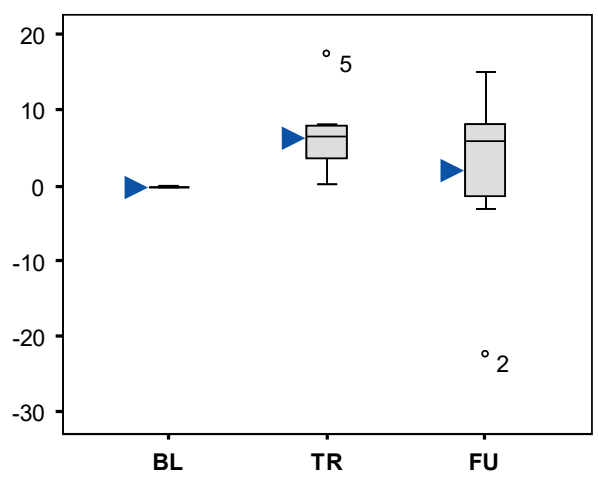

$2 \mathrm{C}$

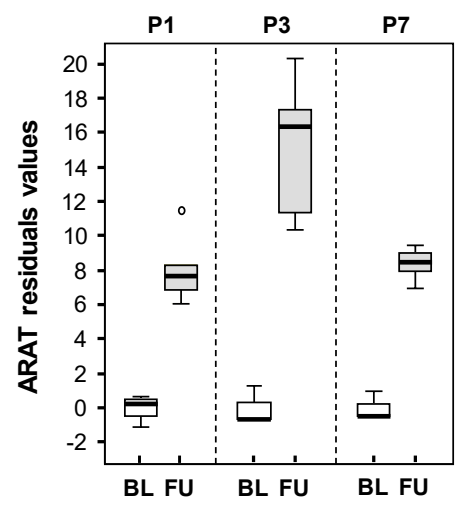

2D

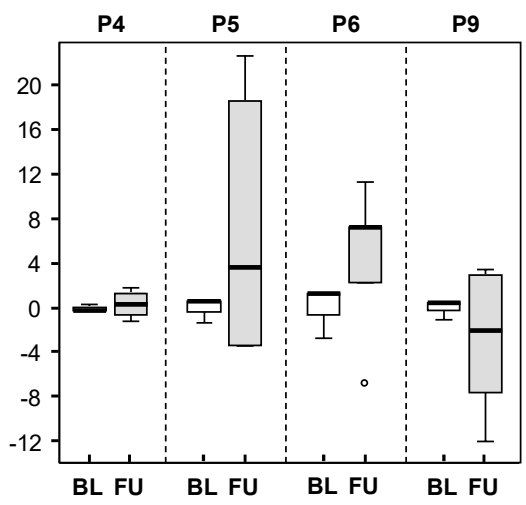

2E

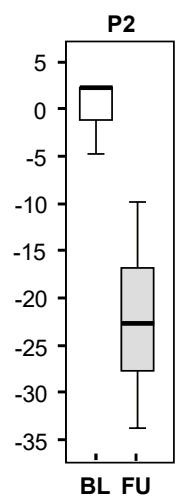

Figure 2. Boxplots of ARAT results. ARAT group results (2A), within-subject averaged residuals for all 3 phases (2B) and within-subject residuals for all subjects for the baseline phase and the follow-up phase (2C-E) are depicted. ARAT = Action Research Arm Test; BL = Baseline; TR = Training; FU = Follow-up; Circles = outlier value; 2 \& $5=$ patient number;

$P=$ patient number; Arrows in Fig. $2 A$ and $2 B$ indicate mean value. Figure $2 A$ : overall: $p=0.001 ; B L-T R: p=0.012$; BL-FU: $p=0.012$; Figure 2B: overall: $p=0.009 ; B L-T R: p=0.001 ; B L-F U: p=0.105$; Figure 2C-E: P1,P3,P7: $p=0.036$; $P 4, P 5, P 6, P 9:$ N.S; $P 2: p=0.036$

Improvement over time, corrected for baseline trends

Representing data of the whole group $(n=8)$, boxplots of the within-subject averaged ARAT time series data for all three phases, i.e. baseline, training and follow-up phase, linearly detrended for the baseline trends, are presented in Figure 2B.

An overall improvement was found for the ARAT results (Kruskal-Wallis test, $p=0.009$ ).

Multiple comparison revealed that ARAT residuals were higher in the training phase, 
relative to those recorded in the baseline phase $(p<0.001)$, whereas no statistical differences were found between the ARAT residuals of the follow-up phase, relative to those recorded in the baseline phase $(p=0.105)$.

\section{Single case time series}

To ascertain how each subject's ARAT score changed over time, boxplots of ARAT time series residuals for the baseline and follow-up phase are presented in Figure $2 \mathrm{C}$ through $2 \mathrm{E}$.

In three patients (P1, P3 and P7) mean (and median) ARAT residuals were higher in the follow-up phase relative to the baseline phase $(p<=0.036)$. In four patients (P4, P5, P6 and P9) no statistically significant improvement in ARAT scores was observed. In one patient (P2) a decrease in mean (and median) ARAT residuals was observed between the baseline phase and follow-up phase $(p=0.036)$.

\section{ABILHAND results}

\section{General improvement over time}

Overall, patients improved over time on the ABILHAND $(P=0.005)$. Multiple comparison revealed that $A B I L H A N D$ results were higher both at follow-up and during the training phase, relative to baseline data $(p=0.017$ and $p=0.012$ respectively). Boxplots of ABILHAND results are presented in Figure 3A. 
3A

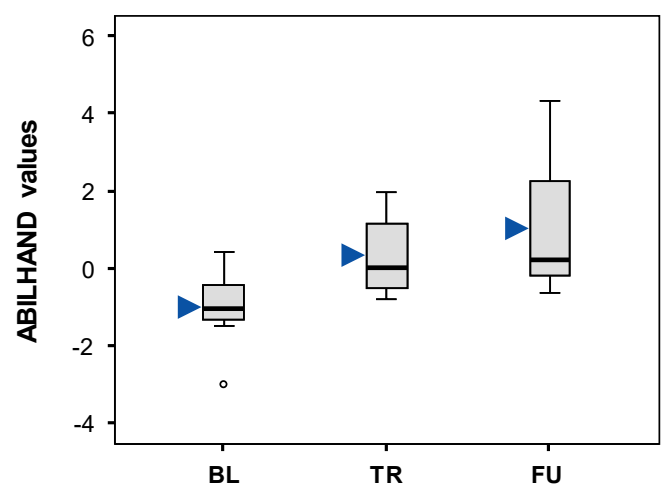

3C

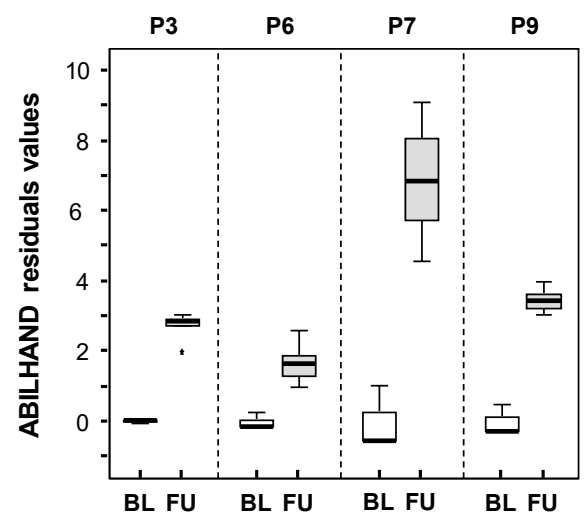

\section{B}

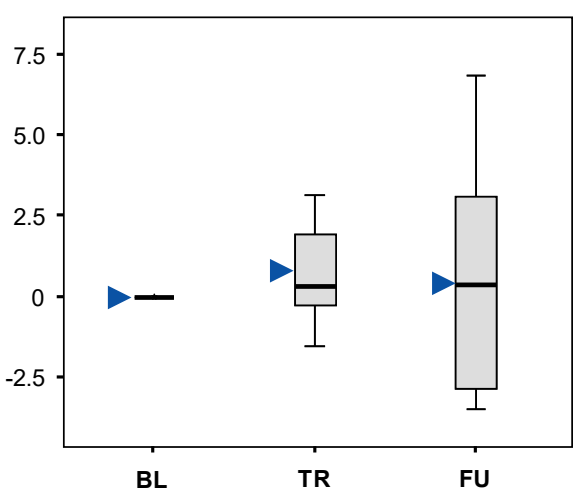

3D

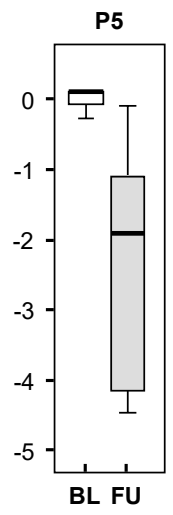

3E

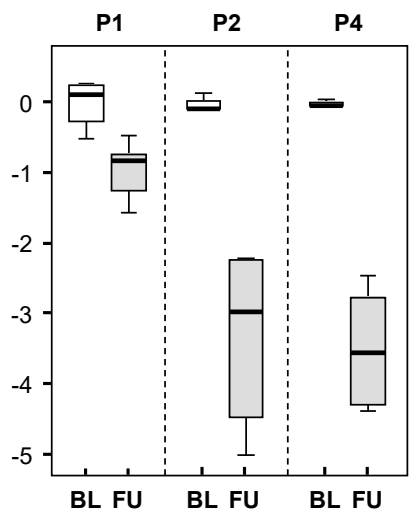

Figure 3. Boxplots of ABILHAND results. ABILHAND group results (3A), within-subject averaged residuals for all 3 phases (3B) and within-subject residuals for all subjects for the baseline phase and the follow-up phase (3C-E) are depicted. $B L=$ Baseline; $T R=$ Training; $F U=$ Follow-up; asterisk = extreme value; $P .$. = patient number; Arrows in Fig. $3 A$ and $3 B$ indicate mean value. Figure $3 A$ : overall: $p=0.005 ; \quad B L-T R: p=0.017 ; \quad B L-F U: p=0.012$. Figure $3 B$ : overall: $p=N S ; \quad B L-T R: p=N S ; \quad B L-F U: p=N S$. Figure 3C-E: P3,P6,P7,P9: $p=0.036 ; P 5: N . S ; P 1, P 2, P 4: p=0.036$.

Improvement over time, corrected for baseline trends

Boxplots of the within-subject averaged ABILHAND time series data for all three phases, linearly detrended for baseline trends, are presented in Figure $3 \mathrm{~A}$. No significant differences in ABILHAND residuals values were found between any of the three phases. 
Single case time series

To ascertain how each subject's ABILHAND score changed over time, boxplots of ABILHAND time series residuals for the baseline and follow-up phase are presented in Figure $3 \mathrm{C}$ through $3 \mathrm{E}$.

In four patients (P3, P6, P7 and P9) mean (and median) ABILHAND residuals were higher in the follow-up phase relative to the baseline phase $(p<=0.036)$. In one patient (P5) no statistically significant improvement in ABILHAND scores was observed $(p=0.071)$. In three patients ( $P 1, P 2$ and $P 4)$ a decrease in mean (and median) ABILHAND residuals was observed between the baseline and follow-up phase $(p<=0.036)$.

IMI results

In Table 3 mean IMI results per participant are displayed.

Table 3. Overview of IMI scores

\begin{tabular}{ll}
\hline IMI item & Mean (sd) \\
\hline & \\
\hline Interest / Enjoyment & $5.4(0.53)$ \\
\hline Perceived competence & $5.9(0.46)$ \\
\hline Effort / Importance & $6.3(0.53)$ \\
\hline Pressure / Tension & $2.1(0.26)$ \\
\hline Value / Usefulness & $6.6(0.21)$ \\
\hline Relatedness & $6.3(0.38)$ \\
\hline
\end{tabular}

Likert scale: 1-7. IMI = Intrinsic Motivation Inventory; sd = standard deviation. 


\section{Discussion}

The aims of this study have been to investigate a) the effects of a dynamic springloaded hand orthosis, adjunct to therapy-as-usual (TAU), on the functional use of the affected upper limb in moderately to severely impaired sub-acute stroke patients, and b) the patients' motivation regarding the usage of a dynamic orthosis.

In general, patients improved considerably on their ability to use their affected arm during and after training, i.e. they improved on both ARAT (measuring patient's capacity) and ABILHAND (measuring patient's perception regarding his actual real life task performance), between baseline phase, training phase and follow up phase. However, as patients were in the sub-acute phase after stroke, this improvement might have (partly) been due to several factors, i.e.; a) spontaneous recovery, b) TAU patients received, c) the use of the dynamic orthosis, or d) a combination of these factors. After removal of the baseline trends, the ARAT group results showed that during the training phase patients improved on their capacity to perform activities. At follow-up, this improved capacity was still clearly present in three patients, indicating that they had benefitted more permanently from the additional dynamic spring-loaded hand orthosis training. These patients improved their dexterity, i.e. gross motor grip function combined with active shoulder movements (abduction and forward flexion). Two additional patients improved on their capacity to perform activities, although results failed to attain statistical significance, whereas in another 2 patients no change was observed. In one patient ARAT improvement, induced during the training phase, had declined post-training, suggesting that, regarding his capacity to perform activities, he did not benefit from the additional orthosis training. In contrast to the results of the ARAT, after correction of the ABILHAND data for spontaneous recovery and TAU no significant additional (group) effect of the dynamic orthosis training was found. However, when assessing the time series of each individual, four patients clearly improved as to their perceived real life task performance post-training, relative to their baseline performance. The four other patients did not improve in this area, but seem to have slightly performed worse at follow-up.

In general, data from both the patients' capacity to perform activities using the affected arm-hand and the patients' perceived real life task performance indicate that those patients who show little progress during baseline, i.e. little improvement at an early stage post-stroke, benefitted more from the adjunct training featuring a dynamic hand 
orthosis. This seems to both hold for patients with an initially low or intermediate functional capacity (low or intermediate ARAT $(<30)$ ). In contrast, for those patients who, during the baseline phase clearly improve (e.g. P2), benefit from the adjunct training with the dynamic hand orthosis seems to be less. In literature, little is reported about the possible added value of dynamic hand orthoses in sub-acute stroke patients. As one of the few, Davenport et al. [28] in an exploratory study, reported proof-ofconcept of using a dynamic spring-loaded orthosis adjunct to TAU. In contrast to our findings, they concluded that patients with a lower baseline ARAT made less change at the end of their intervention. However, in their study effects of TAU and spontaneous recovery were not taken into account, thereby obscuring (and overestimating) any effect of dynamic hand orthoses used.

As to user experience, also encompassing elements of motivation and usability, as measured with the IMI, patients rated the use of the dynamic spring-loaded arm orthosis very favourably at the end of the study. All patients were highly motivated to use the orthosis during training sessions. They felt confident using the orthosis and working with it in an intensive training program, whereas perceived pressure or tension to achieve certain goals or marks was relatively low. Even patients who did not benefit as much as expected from the adjunct orthosis training, anecdotally, reported a higher level of acceptance because they felt "... having done everything that could be done during this period of training".

\section{Considerations and future research}

Despite the seemingly wide variance between subjects, across measures used, the baseline-corrected time series of the individual patients showed similarities between categories of patients, i.e. patients with low initial progress as to arm-hand performance seem to benefit more from the adjunct training provided. Future research should further focus on patient characteristics that may identify these patients as early as possible. Research into specific training adjunct to TAU in sub-acute stroke patients is always methodologically challenging, because of the (speed of the) natural or therapy-induced recovery processes that take place, and the limited time window within the sub-acute stage in which valid data on this topic may be acquired. In our investigation we tried to model this recovery, in order to identify the unique contribution of the adjunct therapy. 
However, our linear model most certainly has led to a) an increasing degree of overestimation of (in our case) spontaneous recovery and TAU effects in the long term post-training, and b) a concurrent increase in within-subject data variance, resulting in underestimation of any effects of the adjunct training. The latter may have been the case especially in $\mathrm{P} 2$.

In general, for all patients, an S-shaped model or at least a model 'levelling off over time' may have better fitted the reality of spontaneous recovery. However, gathering enough data to adequately fit such a model would have necessitated a much longer baseline phase, which, in turn, would inevitably have compromised the sub-acuteness of the patients' status during the ensuing intervention phase. Future research should focus on optimizing this model to better contrast spontaneous recovery with intermediate and long-term effects of (adjunct) therapy.

\section{Declaration of interest}

The authors declare that they have no competing interests. 


\section{References}

1. Landelijke Medische Registratie [Dutch National Medical Registration database]. [Internet]. http://cognosserver.prismant.nl/cognos7/. 2010.

2. Heller A, Wade DT, Wood VA, Sunderland A, Hewer RL, Ward E. Arm function after stroke: measurement and recovery over the first three months. J Neurol Neurosurg Psychiatry. 1987;50(6):714-9. PubMed PMID: 3612152.

3. Sunderland A, Tinson D, Bradley L, Hewer RL. Arm function after stroke. An evaluation of grip strength as a measure of recovery and a prognostic indicator. J Neurol Neurosurg Psychiatry. 1989;52:1267-72.

4. Nakayama H, Jorgensen HS, Raaschou HO, Olsen TS. Recovery of upper extremity function in stroke patients: the Copenhagen Stroke Study. Archives of physical medicine and rehabilitation. 1994;75(4):394-8. Epub 1994/04/01. PubMed PMID: 8172497.

5. Nijland RH, van Wegen EE, Harmeling-van der Wel BC, Kwakkel G. Presence of finger extension and shoulder abduction within 72 hours after stroke predicts functional recovery: early prediction of functional outcome after stroke: the EPOS cohort study. Stroke; a journal of cerebral circulation. 2010;41(4):745-50. Epub 2010/02/20. doi: 10.1161/strokeaha.109.572065. PubMed PMID: 20167916.

6. Kwakkel G, Kollen B, Lindeman E. Understanding the pattern of functional recovery after stroke: facts and theories. Restor Neurol Neurosci. 2004;22(3-5):281-99. PubMed PMID: 15502272.

7. Verheyden G, Nieuwboer A, DeWit L, Thijs V, Dobbelaere J, Devos H, et al. Time course of trunk, arm, leg, and functional recovery after ischemic stroke. Neurorehabil Neural Repair. 2008;22:173 - 9.

8. van Kuijk AA, Pasman JW, Hendricks HT, Zwarts MJ, Geurts AC. Predicting hand motor recovery in severe stroke: The role of motor evoked potentials in relation to early clinical assessment45-51. Neurorehabil Neural Repair. 2009;23:45-51.

9. Levin M, Kleim J, Wolf S. What do motor "recovery" and "compensation" mean in patients following stroke? Neurorehabil Neural Repair. 2009;23(4):313-9. PubMed PMID: 19118128.

10. Wolf SL, Winstein CJ, Miller JP, Thompson PA, Taub E, Uswatte G, et al. Retention of upper limb function in stroke survivors who have received constraint-induced movement therapy: the EXCITE randomised trial. Lancet Neurol. 2008;7(1):33-40. PubMed PMID: 18077218.

11. Barclay-Goddard RE, Stevenson TJ, Poluha W, Thalman L. Mental practice for treating upper extremity deficits in individuals with hemiparesis after stroke. Cochrane Database Syst Rev. 2011:CD005950. 
12. Verbunt JA, Seelen HA, Ramos FP, Michielsen BH, Wetzelaer WL, Moennekens M. Mental practice-based rehabilitation training to improve arm function and daily activity performance in stroke patients: a randomized clinical trial. BMC neurology. 2008;8:7. PubMed PMID: 18405377.

13. Timmermans AA, Spooren AF, Kingma H, Seelen HAM. Influence of task-oriented training content on skilled arm-hand performance in stroke: a systematic review of non-CIMT trials. Neurorehabil Neural Repair. 2010;24(9):858-70.

14. Balasubramanian S, Klein J, Burdet E. Robot-assisted rehabilitation of hand function. Current opinion in neurology. 2010;23(6):661-70. Epub 2010/09/21. doi: 10.1097/WCO.0b013e32833e99a4. PubMed PMID: 20852421.

15. Timmermans AA, Seelen HAM, Geers RP, Saini PK, Winter S, Te Vrugt J, et al. Sensorbased skill training in chronic stroke patients: results on treatment outcome, patient motivation and system usability. IEEE Transactions on Neural Systems and Rehabilitation Engineering. 2010;18(3):284-92.

16. Langhorne P, Bernhardt J, Kwakkel G. Stroke rehabilitation. Lancet. 2011;377:1693 - 702.

17. Hayward K, Barker R, Brauer S. Interventions to promote upper limb recovery in stroke survivors with severe paresis: a systematic review. Disability and rehabilitation. 2010;32(24):1973-86. PubMed PMID: 20964563.

18. Timmermans AA, Seelen HA, Willmann RD, Kingma H. Technology-assisted training of arm-hand skills in stroke: concepts on reacquisition of motor control and therapist guidelines for rehabilitation technology design. Journal of Neuroengineering and Rehabilitation. 2009;6:1. PubMed PMID: 19154570.

19. Mehrholz J, Hadrich A, Platz T, Kugler J, Pohl M. Electromechanical and robot-assisted arm training for improving generic activities of daily living, arm function, and arm muscle strength after stroke. Cochrane Database Syst Rev. 2012;6.

20. Hsieh YW, Wu CY, Liao WW, Lin KC, Wu KY, Lee CY. Effects of treatment intensity in upper limb robot-assisted therapy for chronic stroke: A pilot randomized controlled trial. Neurorehabilitation and Neural Repair. 2011;25:503-11.

21. Kwakkel G, Kollen BJ, Krebs HI. Effects of Robot-Assisted Therapy on Upper Limb Recovery After Stroke: A Systematic Review. Neurorehabil Neural Repair. 2008;22(2):111-21. PubMed PMID: 17876068.

22. Riener R. Robot-aided rehabilitation of neural function in the upper extremitiea. Acta Neurochir Suppl. 2007;97(465-71).

23. Lannin NA, Ada L. Neurorehabilitation splinting: Theory and principles of clinical use. NeuroRehabilitation. 2011;28:21-8. 
24. Lannin NA, Cusick A, McCluskey A, Herbert RD. Effects of splinting on wrist contracture after stroke: A randomized controlled trial. Stroke; a journal of cerebral circulation. 2007;38:111-6.

25. Tyson SF, Kent RM. Orthotic devices after stroke and other non-progressive brain lesion. Cochrane Database Syst Rev. 2009;CD003694.

26. Davenport S. Recovery with grasp? A report on a preliminary study investigating the short and medium term effects of the SaeboFlex (FTM System) on chronic post stroke patients with residual upper limb deficit. Synapse. 2005;(spring):12-5.

27. Butler A, Blanton S, Rowe V, Wolf $S$. Attempting to improve function and quality of life using the FTM Protocol: Case report. J Neurol Phys Ther. 2006;30(148 - 56).

28. Davenport S, Brogden S, Cairns M. Clinical considerations from a study exploring the role of the SaeboFlex dynamic hand orthosis as an adjunct to therapy with acute and subacute stroke patients. 2008;(Harrogate, UK).

29. Wolf S, Winstein C, Miller J, Taub E, Uswatte G, Morris D, et al. Effect of constraintinduced movement therapy on upper extremity function 3 to 9 months after stroke: the EXCITE randomized clinical trial. Jama. 2006;296(17):2095-104. PubMed PMID: 17077374.

30. Jones F, Partridge C, Reid F. The Stroke Self-Efficacy Questionnaire: Measuring individual confidence in functional performance after stroke. Journal of Clinical Nursing. 2008;17:244-52.

31. Barlow D, Nock MK, Hersen M. Single case experimental designs: strategies for studying behavior change. Cambridge: Pearson Publishing; 2008.

32. Wulf $\mathrm{G}$, Shea $\mathrm{C}$, Lewthwaite R. Motor skill learning and performance: a review of influential factors. Med Educ. 2010;44(1):75-84. PubMed PMID: 20078758.

33. Kruitwagen-van Reenen ET, Post MW, Mulder-Bouwens K, Visser-Meily JM. A simple bedside test for upper extremity impairment after stroke: validation of the Utrecht Arm/Hand Test. Disabil Rehabil. 2009;31(16):1338-43.

34. Lemmens RJ, Timmermans AA, Janssen-Potten YJ, Smeets RJ, Seelen HA. Valid and reliable instruments for arm-hand assessment at ICF activity level in persons with hemiplegia: a systematic review. BMC neurology. 2012;12:21. Epub 2012/04/14. doi: 10.1186/1471-2377-12-21. PubMed PMID: 22498041; PubMed Central PMCID: PMCPMC3352056.

35. Hsueh IP, Hsieh CL. Responsiveness of two upper extremity function instruments for stroke inpatients receiving rehabilitation. Clinical Rehabilitation. 2002;16(617-24).

36. Lyle RC. A performance test for assessment of upper limb function in physical rehabilitation treatment and research. Int J Rehabil Res. 1981;4(4):483-92. PubMed PMID: 7333761. 
37. Weerdt de WJG, Harrison MA. Measuring recovery of arm-hand function in stroke patients: a comparison of the Brunnstrom-Fugl-Mayer test and the Action Research Arm test. Physiotherapy Canada. 1985;37:65-70.

38. van der Lee JH, Roorda LD, Beckerman H, Lankhorst GJ, Bouter LM. Improving the Action Research Arm test: a unidimensional hierarchical scale. Clinical Rehabilitation. 2002;16(6):646-53. PubMed PMID: 12392340.

39. Ashford S, Slade M, Malaprade F, Turner-Stokes L. Evaluation of functional outcome measures for the hemiparetic upper limb: a systematic review. J Rehabil Med. 2008;40(10):787-95. PubMed PMID: 19242614.

40. Wang TN, Lin KC, Wu CY, Chung CY, Pei YC, Teng YK. Validity, responsiveness, and clinically important difference of the ABILHAND questionnaire in patients with stroke. Archives of Physical Medical Rehabilitation. 2011;92:1086 - 91.

41. Deci EL, Eghrari $H$, Patrick BC, Leone DR. Facilitating internalization: the selfdetermination theory perspective. J Pers. 1994;62(1):119-42. PubMed PMID: 8169757.

42. Johnson MJ, Loureiro RC, Harwin WS. Collaborative tele-rehabilitation and robotmediated therapy for stroke rehabilitation at home or clinic. Intel Serv Robotics. 2008;1:109-21.

43. Tsigilis N, Theodosiou A. Temporal stability of the intrinsic motivation inventory. Perceptual and motor skills. 2003;97(1):271-80. Epub 2003/11/08. doi: 10.2466/pms.2003.97.1.271. PubMed PMID: 14604050. 


\section{Chapter}

General Discussion 



\section{Aim of this thesis}

The overall aim of the work presented in this thesis was to develop and evaluate a systematic arm-hand rehabilitation approach in order to offer clinicians knowledge and tools in systematically coaching sub-acute stroke patients who suffer from a moderately to severely affected arm and hand. To achieve this aim, the present thesis addressed three relevant topics in arm-hand rehabilitation in stroke. Firstly, a Concise Arm-hand Rehabilitation Approach in Stroke (acronym: CARAS) was presented as an in detail-described form of 'therapy-as-usual' for arm-hand rehabilitation aimed at a heterogeneous group of sub-acute stroke patients typically seen in day-to-day stroke rehabilitation. Secondly, results regarding the rate of improvement of arm-hand function (AHF), arm-hand capacity, self-perceived arm-hand skill performance (AHSP) and actual arm-hand use in patients from the aforementioned group, who received CARAS, were presented. Thirdly, two non-actuated dynamic hand-orthoses as well as the application of botulinum-toxin adjunct to 'therapy-as-usual', all applied to facilitate the functional use of the moderately to severely impaired hand during and after rehabilitation, were systematically evaluated. In this chapter the main research findings are discussed, as are a number of methodological considerations. In the final paragraph, future perspectives and directions are discussed from both a clinical and a research perspective. 


\section{Main findings and results}

\section{CARAS}

Rationale behind the development of CARAS

Solving arm-hand deficits in stroke survivors necessitates stepwise clinical procedures and is considered to be challenging. Unfortunately, comprehensive descriptions of arm-hand interventions, offered in stroke rehabilitation, often are lacking. Sufficiently detailed information regarding therapy content (activities, therapy approaches), settings and intensity often is not provided and/or may vary highly [1-3], which makes these interventions to be considered 'black boxes' [4-6]. Apart from information regarding therapy time, expressed in terms of duration and frequency, studies rarely provide details of arm-hand rehabilitation programmes used, making study replication difficult and obscuring causality as to (e.g.) therapy effectiveness.

In literature, the majority of arm-hand rehabilitation programs is presented as fixed intervention protocols $[7,8]$ without sufficiently taking into account the patient's individual possibilities and needs. These intervention protocols frequently target specific (study) sub-populations and thus, are less applicable in heterogeneous patient populations commonly seen in contemporary stroke rehabilitation practice. Most protocols lack information about how therapy content relates to the patient's goals, and what actually occurs in patients admitted to arm-hand rehabilitation [4, 5, 9-11]. Besides, to allow quick adaptations to novel and effective innovations without disturbing clinical routines, this demands an easy-to-organise modular therapy regime [12]. However, most arm-hand treatment protocols as presented in the literature, are not organized in a way that allows content of training to be easily replaced by other, often novel, training methods without disturbing routines in daily treatment planning.

The aforementioned shortcomings in therapy descriptions have led to the development of CARAS. It is based on a number of principles, discussed below, in order to guide therapists in organizing and structuring arm-hand treatment in sub-acute stroke survivors admitted to a rehabilitation setting. CARAS has been designed as a groupbased, yet individually adjustable, training program and has been implemented as a substitute for conventional individual arm-hand therapy [13]. 


\section{CARAS' main constructs}

\section{Accommodating patient group heterogeneity}

During therapy, sub-acute stroke patients with severe, moderate or mild arm-hand impairments are stratified according to the Utrecht Arm Hand Test (UAT) [14], an 8 point ordinal scale, based on the stages of recovery as described by Brunnstrom [15]. The UAT objectively differentiates between the presence of distal (hand-wrist) selectivity ((UAT score: 2 -7, i.e. moderately impaired (UAT score 2-3) and mildly (UAT score 4-7) impaired)), and the absence of selectivity, i.e. severely impaired (UAT score: $0-1)$. In contrast to persons with a severely affected arm-hand, moderately and mildly impaired persons are allocated to a highly intensive, task-oriented training regime, i.e. Part 2 of CARAS. This means that also patients with a moderately impaired arm-hand are exposed to task-oriented and functional arm-hand training in order to regain dexterity, despite the fact that a substantial part of them lack finger extension at the initial phase of training. This is done for two reasons: 1) Sub-acute stroke patients who enter CARAS are still in the $8-12$ weeks post-stroke onset phase and regaining dexterity is possible within this (limited) time window [16]; 2) Being actively engaged during therapy provides the patient with valuable insights into any progress made in his attempts to regain dexterity, which, in turn may stimulate patient's motivation and fuel his sense of 'being in control again'.

If in patients with a severely affected arm-hand (UAT 0-1) no neurophysiological recovery takes place, no functional gain is foreseen, and they will be unable to use their affected arm-hand in daily activities. It is not useful to train these patients under the same practice conditions and as intensively as patients admitted to program 2 and 3. In program 1, i.e. Part 1 of CARAS, these patients receive training which consists of education, exercises on how to position the arm-hand, exercises to avoid discomfort, maintain joint mobility, and maintain muscles/tendons in an optimal condition, exercises to provoke voluntary movement where possible, and training how to use supportive tools. 


\section{Principles of motor learning}

Task-oriented training, based on motor learning principles, is the gold standard for post-stroke rehabilitation, both in current therapeutic approaches and in clinical research [17].Theoretical constructs of the task-oriented training part of CARAS encompass a clinical reasoning model combined with a hypothetico-deductive clinical reasoning method $[18,19]$ to define relevant motor control problems. The training part consists of re-learning motor abilities of the affected arm-hand, by applying suitable training conditions, learning approaches [20] and self-efficacy principles [21], tailored to a patient's needs and specified to the goals set.

The clinical reasoning model is applied in order to guide therapists in:1) Identifying in a systematic way the nature and cause of abnormal movements, as well as factors affecting motor learning and recovery of arm-hand function; 2) Establishing and implementing an intervention plan, tailored to the health condition of the individual, in order to facilitate motor learning in the most optimal way. If both steps are performed correctly, patients with a moderately or mildly impaired arm-hand and a substantial part of patients with a severely affected arm-hand may feel more confident in their ability to perform a task. The hypothetico-deductive clinical reasoning method is used to assist clinicians in their analysis on how arm-hand impairments are related to functional deficits and how to distinguish between interventions designed to improve AHF and AHSP, and reduce underlying disabilities [19].

\section{Application of self-efficacy principles}

Self-efficacy principles are applied to stimulate the patient's awareness of the capacity of the affected arm and hand in a positive way. Creating perspective on how and when to use the affected hand in daily performance at an early stage during rehabilitation may encourage patients to push their limits by exploring actual arm-hand use [21-23]. Ultimately, patients facilitate themselves in creating an optimal retention of learning to use the full potential of the affected hand post-rehabilitation. For this reason, patients are exposed to therapy sessions in which they work as independently as possible, and therapeutic assistance during therapy sessions is gradually phased out. 
Swift systematic incorporation of technology and new evidence-based training elements

During arm-hand rehabilitation interventions, patients are enabled to practice tasks which are important to them. Setting and updating of realistic goals is done within the boundaries of residual voluntary (neural) control of their affected arm-hand. Once admitted to an arm-hand rehabilitation regime, patients have to work towards daily skills of progressive difficulty and intensity. To deliver the most appropriate and effective treatment necessitates an overview of current practice and regular updates of treatment as new insight or new evidence emerges.

Interesting developments encompass, amongst others, pharmacological interventions like botulinum toxin [2, 24], virtual reality [25], brain-machine interfaces [26], electrical stimulation [27, 28] and technology-assisted devices like rehabilitation robots $[29,30]$ or dynamic hand orthoses [31], applied to complement conventional arm-hand rehabilitation therapy in stroke patients.

In daily practice, arm-hand rehabilitation interventions may be combined and may be used synergistically in order to speed up functional recovery of the affected arm-hand. For instance, assistive technologies, in conjunction with electrical stimulation, may be applied in a task-oriented training regime [32,33]. The application of these hybrid forms of therapy are upcoming in day-to-day clinical practice [34], and, depending on progressions in AHF and/or AHSP achieved, such combinations may change during the rehabilitation process.

Incorporating new insights in clinical practice remains limited [12] due to several factors like: 1) Difficulties to change daily care routines which demands flexibility from therapists and care delivery structures [35]; 2) The unknown superiority over standard care; 3) The (dis-) comfort of therapists with the use of novel technologies [36]; 4) The speed of developments in e.g. technology, and 5) The increasing workload in healthcare [35].

Other recent developments encompass pharmacological agents like, for instance, botulinum toxin [2, 24] and assistive technologies [29, 30, 37] as applications to complement conventional arm-hand rehabilitation therapy in stroke patients. Botulinum toxin is frequently applied adjunct to arm-hand rehabilitation in order to reduce pain or to create motor improvements [38-41]. Nevertheless, uncertainty exists regarding its 
role in improving motor dysfunction after stroke [42], and for which type of stroke patients it may be beneficial. This may lead to disagreement among clinicians regarding when and where botulinum toxin should be applied [43].

Application of assistive technologies adjunct to conventional arm-hand rehabilitation may support the therapist in increasing therapy intensity and dosage, and may reduce the physical burden on therapists [44, 45]. However, despite rapid advances in the development of assistive technologies, there is a lack towards implementing these devices in rehabilitation due to several factors like, for instance, their added-value regarding therapeutic effectiveness [46], cost-effectiveness, fun to use and robustness [45].

To investigate the added-value of novel insights before they are implemented in rehabilitation would be the most optimal situation to support high quality care. However, to keep up with the latest evidence, and to systematically evaluate novel insights swiftly, is difficult given the speed of developments in e.g. technology [35, 47] and the evaluation methodology necessary to assess the effectiveness of new developments [48, 49]. Furthermore, once a development has been proven effective, an implementation strategy must be used to facilitate its actual application in the clinic. In order to respond to new developments adequately, CARAS' time table consists of easy to replace schedules fitted in time blocks in which the type of training is defined. The training content and therapy time can be adjusted without the therapist having to rearrange treatment time schedules.

CARAS' modularity and monitoring procedures can also facilitate the use of (parts of) other arm-hand therapy protocols, like Constrained-Induced Movement Therapy (CIMT) [50], to be integrated in CARAS. Depending on the patient's needs, a (modified form of) CIMT can be implemented on a regular basis without having to resort to major (systemic) alterations in daily routines as for instance therapy time.

The use of mobility aids, like the application of dynamic hand orthoses or the application of pharmacological interventions can be easily applied in CARAS' part 2. Especially when these interventions assist sub-acute stroke patients with no or minimal voluntary finger flexion to execute a functional grasp independently in such a way that they are facilitated to train as independently as possible. Also, several variations of hybrid forms of therapy may be applied and evaluated, like the application of electrical 
stimulation adjuvant to a dynamic orthosis to provoke gross motor grasp-and-release tasks in moderately to severely affected patients admitted to the task-oriented training part of CARAS.

\section{Intensity of arm-hand training}

Stroke patients who do not regain voluntary finger extension early post-stroke may have a poor prognosis regarding the improvement of arm-hand capacity [51]. The proportional functional recovery from motor impairments in the affected arm-hand depends on the process of neurophysiological recovery [52-54] and may depend more on corticomotor tract integrity than on the amount of time spent on arm-hand therapy $[55,56]$. However, proponents of high intensity training suggest that the dose of activity-related arm-hand training is a critical factor contributing to successful rehabilitation $[1,57,58]$. Ward et al.

even recommended to invest in much higher doses of treatment than currently attempted [59].

In the general debate about the most appropriate dosage of arm-hand rehabilitation, moderate-quality evidence was provided towards a beneficial effect for greater doses (57 hours of therapy) compared to smaller doses (24 hours of therapy) [1, 60] in repetitive practice training interventions. In patients with a mildly impaired arm-hand who are admitted to CARAS, the total therapy time is almost equivalent to other armhand rehabilitation programs applied in stroke patients with a mildly impaired arm-hand [62-64]. Patients with a moderately affected arm admitted to the CARAS gross motor grip performance program receive at least three sessions of 90 minutes weekly for twelve consecutive weeks (54 hours of therapy)[13]. They receive less therapy time compared training regimes presented in two recently published studies by Ward et al [59] and by Daly et al. [57]. Ward et al. reported a total of 90 hours of therapy for patients with a moderately affected arm-hand [59]. However, their classification of a moderately impaired arm-hand differs from the classification used in CARAS. The median values at admission of the Action Research Arm Test (ARAT) ARAT and Fugl Meyer Motor Assessment (FMA) of the total group of patients who participated in their study were 18 points and 26 points, respectively. Daly and colleagues provided 300 hours of treatment, divided over 12 weeks [57]. The median value of the FMA presented in their study was 21.8 points, which, from a motor recovery perspective, is 
comparable to the data from the moderately affected group of patients admitted to CARAS, with a median score on the ARAT of 4 points and a FMA score of 22 points [61]. Unfortunately, given the diversity between the aforementioned studies regarding content, intensity and patient populations, it remains difficult to infer on the exact differences in effectiveness between these studies.

The search for the most optimal dosage of therapy time for patients remains difficult [1], especially when interventions (and time required to perform these interventions) used in most studies are not tailored to individual patient's needs. Depending on the status of the individual patient, the optimal dose of therapy time and therapy intensity may fluctuate during the course of the arm-hand rehabilitation program.

\section{Arm-hand performance during and after CARAS}

Whereas arm-hand rehabilitation may reduce arm-hand impairments and enhance a person's functional ability to use the hand in daily practice [59,65], it is known that in some patients arm-hand performance may deteriorate once they have left the training program [66-70]. However, it is not well understood at which rate any deterioration (or improvement) occurs post-rehabilitation, nor in which patient categories, i.e. patients with a certain level of arm-hand impairment severity, this is most prominent.

In view of the evaluation of CARAS, which was implemented in regular care as a form of therapy-as-usual, it was important to identify and objectively quantify any changes in the course of arm-hand performance during and after the rehabilitation phase.

A second question was to what extent the rate of improvement or deterioration (over time) of AHF, AHSP and actual arm-hand use differed between three subgroups of stroke patients, i.e. patients with either a severely, moderately or mildly impaired armhand during and after their rehabilitation phase involving CARAS.

As has been outlined in chapter 1, AHF has been measured using the Fugl-Meyer Motor Assessment (FMA) [71] and grip strength by using dynamometry [72]. AHSP, encompassing both capacity and perceived performance, has been evaluated with the Action Research Arm test (ARAT) [73] and ABILHAND [74]. Actual arm-hand use, as part of AHSP, has been measured using accelerometry. 
When comparing rate of progression among stroke sub-groups, the smallest gains as to AHF, AHSP and actual arm use were observed in the severely affected part of the study population $[61,75]$. Due to the absence of any voluntary dexterity (UAT 0-1) at approximately 30 days post-stroke, it was not feasible to admit these patients to the high-intensive task-oriented training (part 2 of CARAS). Instead, they were admitted to the 'Taking care and prevention' (part 1 of CARAS) to learn to keep their severely affected arm-hand in an optimal condition. From a functional perspective, improvements made in this low functional group were less relevant, as the functional benefits, i.e. integrating the affected hand in daily task performance, were minimal. Although not clinically relevant, significant improvements at AHF and AHSP capacity and self-perceived performance level were observed during and after the rehabilitation phase, except for grip strength. In contrast, intensity of unimanual arm-hand use on the affected side sharply dropped during the rehabilitation phase and remained low in the post-rehabilitation phase. Intensity during bimanual use as well the duration of armhand use improved during the rehabilitation period and remained stable, yet remained relatively low in post-clinical phase, compared to moderately and mildly affected patients. Improvements in Intensity during bimanual arm-hand-use may have been caused by patients performing exercises to keep the affected arm and hand supple and pain free. In order to perform these exercises correctly, the non-affected hand guides the affected arm and hand.

Both sub-acute stroke patients with a mildly or moderately impaired hand, admitted to CARAS' part 2, task-oriented training program, improved significantly at AHF and AHSP level during the rehabilitation phase. Also their level of Intensity-of-arm-handuse during bimanual activities improved significantly during rehabilitation and these levels were maintained during the post-rehabilitation phase. During the training phase intensity to use the affected hand in unimanual activities dropped in both the mildly and moderately affected group. In exact the same time, during bimanual activities, the Intensity-of-arm-and-hand-use on the affected side increased. This phenomenon could be explained by the fact that patients learn to co-use their affected arm-hand more during bimanual performance instead of predominantly using the non-affected armhand. Largest gains as to AHF and AHSP and actual arm use, both during and after the rehabilitation phase, were observed in stroke survivors with an initially moderately 
affected arm-hand. This means that patients who lack any dexterity, i.e. finger extension at the time of admission to the task-oriented training program, still have an ability to generate and maintain significant improvements in AHF, AHSP and actual arm-hand-use, as also has been observed by others [76].

Duration-of-use of the affected hand improved in both the moderately and mildly impaired groups during exact the same period of time and results were maintained in the post-clinical phase. In both these groups the ratio of Intensity-of-arm-hand-use between the affected and non-affected side during bimanual activities increased significantly during the rehabilitation period and remained unchanged in the postrehabilitation phase.

Although in all three subgroups most people improved as to AHF and AHSP capacity in the post-clinical phase, a small proportion of people showed a deterioration, though this was only a minor change. In all groups the number of patients who experienced a deterioration regarding perceived performance in the post-clinical phase was higher relative to the number of patients who showed a deterioration on the objectively quantified performance measures like the ARAT, FM, and grip-strength. This implies that patients' ability to overcome difficulties during daily arm-hand performance tasks is not always experienced as being present. It also must be taken into account that, whereas the ABILHAND captures patients' perceived performance at general activity level, patients often train activities that are very specific to them, which may not be well represented in the more general domain of the ABILHAND. When perceived ability is rated as being low, this may also reflect a low level of self-efficacy as was also reported by Korpershoek et al. [77], which implies that a patient may not be encouraged to use his affected arm-hand in exploring certain hobbies or activities to the same extent as persons with a higher level of self-efficacy $[22,78]$. Post- stroke depression, pain, or socio-cultural factors may also have a negative impact on the patients' sense of selfefficacy and may influence perceived performance negatively [79].

The course of long-term motor function and functional recovery of the affected armhand in post-stroke phase is described by several authors [80-83]. Also, outcomes expressed in AHF, AHSP and/or actual arm-hand-use, during and after the rehabilitation phase, of sub-acute stroke survivors who followed a specific arm-hand intervention program during the rehabilitation phase have been described elsewhere 
$[17,63,64,66,84,85]$. In most cases, results with respect to outcomes on arm-hand improvement are described in moderate to mildly impaired patients using narrow inclusions and are often inconclusive. Meyer et al. (2015) revealed a significant deterioration in arm function between 6 months and 5 years after stroke [70]. Doman and Rand observed a significant improvement in motor and functional ability of subacute stroke survivors with a moderately to mildly affected hand, but no significant increase of daily arm-hand use in post-rehabilitation phase [80, 86]. Some authors stated that the non-affected hand is used predominantly while the affected hand is disregarded, developing so-called learned non-use [87], and thereby prolonging the inability to perform (certain) meaningful activities and reducing participation in society, especially in patients with a severely to moderately affected hand. In view of the aforementioned inconclusive evidence provided in literature, the AMUSE study was performed, gauging patients' arm-hand performance at all levels of the ICF, both during and after the rehabilitation phase $[61,75]$. Based on the results of the comprehensive longitudinal database compiled during the AMUSE study, it can be stated that subacute stroke patients who participated in an arm-hand training regime featuring CARAS, program 2 and program 3, improved on AHF, AHSP and actual arm-hand use during the rehabilitation phase. Patients with a mildly impaired arm-hand maintained these levels during the post-rehabilitation phase, while moderately impaired patients even increased further during the post-rehabilitation period. 


\section{Interventions adjunct to CARAS}

Adjunct to CARAS, the added-value of two dynamic hand orthoses, the Saeboflex and Saeboglove, respectively, and one pharmacological intervention involving the use of Abotulinum toxin-A, have been investigated. All three interventions were applied in order to facilitate voluntary movements of the moderate to severely affected hand towards grasp-and-release tasks during task-oriented training. In the following paragraph, results of these additional interventions are discussed.

\section{Dynamic hand orthoses}

In two separate studies, the added-value of two non-actuated dynamic hand orthoses, i.e. the Saeboflex, a spring-loaded hand orthosis (study 1), and the Saeboglove, a tensioner-assisted hand orthosis, combined with electrical stimulation (ES) (study 2) was investigated in conjunction with CARAS' program 2, gross motor grip performance program [32,33]. The study participants who participated in these studies were all subacute stroke patients with a moderately to severely affected hand. ARAT values, as primary outcome measure taken at baseline in both studies showed that in a substantial part $(88 \%)$ of the participants no dexterity was observed. These studies featured a so-called single case experimental design with a baseline phase, an intervention phase and a follow-up phase. At point of admission, this particular group of patients was not able to work actively and independently with their affected arm and hand during training sessions. Prior to the intervention phase, patients from both studies were equipped with either one of the two dynamic hand orthoses.

At group level, patients in both studies showed significant improvements at arm-hand capacity during and after the training, as measured with the ARAT. In study 1 , the posttraining phase showed a decline of arm-hand capacity in a major part of the study participants. In study 2 , at group level, improvements between the intervention phase and follow-up phase were considerable, but not significant.

Both studies show that combining a dynamic hand orthosis (study 1), or a dynamic hand orthosis with ES (study 2) in conjunction with CARAS' task-oriented training program create training conditions which lead to improvements, although not always statistically significant and sustainable, in arm-hand capacity in patients with an uncertain prognosis regarding regaining dexterity. 


\section{Botulinum Toxin}

The presence of spasticity in stroke patients with a moderately or severely affected arm-hand may limits the development of voluntary movements in the affected arm and hand during rehabilitation and functional recovery may be delayed $[2,88]$. To determine the added-value of early spasticity reduction to improve AHF and AHSP in sub-acute stroke patients with a moderately to severely affected hand, all study participants who developed early signs of spasticity were treated with abobotulinum toxin-A (ABoNt-A) in the first 5 weeks of CARAS' gross motor grip performance program. After baseline trend correction, adjusting for spontaneous recovery and therapy-as-usual effects, group level data did not provide statistically significant results with respect to the application of ABoNt-A as an additional improvement of AHF and AHSP adjunct to therapy-as-usual, i.e. CARAS. However, at individual level, after baseline trend correction, 7 out of 10 patients improved on AHF, whereas 6/10 patients improved on AHSP. For sub-acute stroke patients who suffer from spasticity and display no dexterity at point of admission to CARAS' task-oriented training part, spasticity reduction may lead to improvements in arm-hand performance.

\section{Clinical implications}

As observed in regular stroke rehabilitation, up to $30 \%$ of the patients copes with a severely impaired arm-hand $[89,90]$ and 33 to $66 \%$ of stroke patients with a paretic arm-hand does not show any recovery on AHF six months after stroke [90]. Furthermore, it has been suggested that stroke patients who do not show any voluntary motor control after 5-9 days post-stroke have a low probability of achieving dexterity [91].

From a clinical point of view, it is of utmost importance to achieve a sufficient level of actual arm-hand performance in home-based situations. Currently, existing evidencebased arm-hand rehabilitation interventions (e.g. constraint-induced movement therapy, robot-assisted therapy) are foremost aimed at stroke patients with a mildly affected arm-hand [8, 92, 93]. For patients who cope with a moderately to severely affected arm-hand, evidence based interventions to improve AHF and AHSP and to maintain a sufficient level of AHF and AHSP post-rehabilitation are still scarce [8, 76, 94-96]. A reason for this may be that many studies aimed at ascertaining the 
effectiveness of arm-hand interventions in stroke patients, strict inclusion criteria were set in order to reduce, for example, within-group variance. This led to a well-defined, yet narrow bandwidth in terms of level of (arm-hand) impairment severity, most often limited to mildly affected stroke patients. From a methodological point of view this may be wise. However, the other (clinical) side of the story is that the development of novel treatments and the study of the efficacy of such treatments in patients with a moderately to severely affected arm-hand, and those that have more complex (co)morbidity, is slow. Perhaps the most urgent problem is that patients with moderate to severe arm-hand impairments are limited, or even unable to execute a functional grasp independently due to insufficient voluntary force generation. For them to participate in regular arm-hand skill training at the start of rehabilitation, working actively and independently with the affected arm-hand, is difficult or even impossible [97, 98]. The challenge lies in developing and implementing novel treatments for this patient category. CARAS has explicitly been designed and shown, to accommodate the heterogeneity of patients presented to rehabilitation centers, yet be specific enough to allow patients with different levels of impairment severity to be able to participate in meaningful arm-hand training.

The vast majority of a heterogonous group of stroke patients with an affected hand who were admitted to CARAS' gross motor grip performance program, improved considerably on AHF and AHSP (arm-hand capacity, self-perceived performance and actual arm-hand use) during rehabilitation. In addition, they were able to maintain these high levels during the post-rehabilitation phase $[61,75]$. Largest gains with respect to improvements in AHF and AHSP occurred in moderately and partly in severely impaired patients. It is most likely that the majority of these patients were able to (re)use their affected arm-hand in home-based situations. Furthermore, the application of an intensive arm-hand rehabilitation in conjunction with assistive devices in moderate to severely affected patients showed that it may be of added-value regarding improvements in AHF and AHSP. It was also shown that a part of moderately to severely affected patients responded well to the combined intervention of arm-hand therapy and ABoNt-A in terms of AHF and AHSP progression. 
From a clinical perspective there are several important aspects to discuss.

Firstly, CARAS can be used as a form of therapy-as-usual applied to a heterogeneous group of stroke patients with a moderately to severely affected arm-hand, typically seen in a regular stroke rehabilitation centre. Patients admitted to CARAS received a welldocumented form of 'therapy-as-usual'. Its theoretical framework will assist clinicians in the clinical management of motor control problems and set-up of training conditions and concomitant motor learning principles. CARAS accommodates appropriate evaluation methods from both the clinical perspective and patient's perspective. Data obtained from our single-armed prospective cohort study using CARAS may be used as reference data both in clinical practice and in future research as a reference database to contrast newly developed training interventions. This is especially important since clearly defining 'therapy-as-usual' (TAU) is a problem in AHSP research in stroke patients. In many studies evaluating newly developed training protocols, each of these new training approaches is contrasted to some kind of TAU, the latter of which may vary widely between clinics and institutes. Even worse, often TAU is not clearly defined at all.

Secondly, in order to deliver effective arm-hand therapy it is important to stratify patients based on their capacity regarding neural organization and recovery [3, 99]. However, recovery profiles of patients with a severely affected arm-hand do not fit in current existing proportional recovery models [53]. It is important to realize that a number of these so-called "non-fitters" may regain dexterity, although this is not mentioned by the authors [100].

The results of this thesis underline the importance of gaining insight in how (dis-)similar arm-hand severity levels, i.e. severely, moderately and mildly affected, are determined by different authors across literature [101-103], and how these levels are used in clinical practice. It is particularly important to realise that these levels are predominantly determined from a research point of view, and hardly ever from the perspective of the (individual) patient's perception. For example, a pianist, diagnosed with a mildly impaired arm and hand referred to CARAS' functional performance program, will most certainly perceive his arm-hand condition as being severely impaired.

The FMA is often used to determine the level of motor impairment of the affected arm and hand [104, 105]. To determine the level of arm-hand capacity, or so-called dexterity, the ARAT is often considered [104]. Some authors have argued that a low 
score (e.g., 10/57 on the Action Research Arm Test) may not reflect a meaningful arm function [90,91]. Based on this arbitrary view, patients are artificially allocated to one of two groups: patients who have no dexterity, and patients who have dexterity, leaving little room for nuances. From a clinical point of view, it is important to note that dexterity is a perquisite for arm-hand performance. However, in some patients who have no dexterity in the initial phase of arm-hand rehabilitation, training may still lead to improved AHF and AHSP levels, and possibly to meaningful daily arm-hand performance.

Easy applicable and valid outcome prediction models for moderately to severely affected stroke patients that may be commonly usable in stroke rehabilitation practice, are still under development [106-108]. Earlier prediction models use rather strict inclusion and exclusion criteria, and predominantly target first-ever mildly to moderately impaired stroke patients [52, 91, 100]. To identify individuals with potential for recovery among those patients who cope with severe (motor) impairments in the sub-acute phase post-stroke is challenging $[106,107]$. This is especially true in clinical rehabilitation settings as clinical outcome measures add little to the ability to predict expected recovery in severely affected patients [55].

The substantial amount of variability in characteristics between stroke patients typically seen in stroke rehabilitation leads to large variability in recovery patterns [100], especially among the more severely impaired patients [109]. This leaves us with the question how to provide the best possible care with respect to arm-hand rehabilitation for sub-acute stroke patients who lack any dexterity at point of admission to rehabilitation. Hoonhorst et al. and Woytowicz et al. suggested to use four distinct armhand impairment classification levels, i.e. severe, severe-moderate, moderate-mild and mild, based on the FMA [101, 102] and ARAT [101]. From a clinical point of view, these four levels allow for more nuances in both the content and set-up of training between the severe and severe-moderate sub-groups. This allows for the accommodation a more differentiated treatment of those who have no dexterity but who, at the initial phase of rehabilitation, have the prospect to regain (some) dexterity. Once admitted to CARAS, patients are stratified based on the severity of their armhand impairment for which the Utrecht Arm-hand Test (UAT) is used [14]. Looking back, it can be stated that this UAT-based classification adequately classified the moderately (UAT score: 2 - 3) and mildly affected patients (UAT score: $4-7$ ). In patients with a severely impaired hand, originally classified with an UAT score of $0-1$ 
at baseline, $25 \%$ ( 7 out of 28 ) showed more than the expected average progression in the affected arm-hand. Pending valid (novel) prediction models for the severely affected patients, the aforementioned findings show that stratification of the patient population based on the severity of arm-hand impairment, i.e. the UAT score, seems to be a viable, pragmatic and practical solution to the allocation of patients to an armhand rehabilitation regime, adjusted to their needs and possibilities.

Thirdly, one of the major challenges in stroke rehabilitation services is to deal with the absent or limited spontaneous use of the affected arm-hand in the patient's natural environment, i.e. at home [110]. The focus of arm-hand rehabilitation is to achieve and maintain actual arm-hand performance, outside the therapeutic environment. The ultimate goal is that the patient's affected arm and hand can be (and is) used to assist in the successful completion of a meaningful task.

Actual arm hand use of the affected arm-hand and what persons actually do with their affected hand is not necessarily correlated with AHF and AHSP measures currently applied in the clinic [111, 112]. In essence, arm-hand therapy is not about regaining or maintaining motor improvements or dexterity, neither it is about 'forcing' patients to perform tasks with their paretic hand using behavioural contracts or immobilising the unaffected arm [113]. Arm-hand therapy is about recognizing improvements in AHF and/or AHSP, which may encourage stroke patients to push their limits and create perspectives to re-use their affected hand more quickly in daily tasks. Once encouraged, patients will facilitate themselves to re-explore their motor capabilities in relation to real life skills and tasks. Ultimately, they create an optimal retention of learning to use the full potential of the affected hand outside therapeutic situations as quickly as possible and, eventually, in post-rehabilitation.

Nevertheless, compared to the rehabilitation regime of stroke survivors with an initially mildly impaired hand, the course towards regaining control over goal-directed voluntary movements of the moderately to severely affected hand and how to organize day-today arm-hand rehabilitation is more difficult. One of the main reasons for this is the variances in (motor) recovery patterns [90], and comorbidity [76] among these patients. To choose the most appropriate approach towards improvements in AHF and AHSP in the latter group demands extensive knowledge with respect to clinical management of motor control problems, training conditions and motor learning principles. It also 
demands extensively more therapy time and therapeutic effort. The design and content of arm-hand training for the moderately to severely affected patient is not 'one-sizefits-all'. It has to be patient-tailored. In the first week of CARAS' gross motor grip performance program, a stepwise clinical management procedure, in conjunction with self-efficacy-enhancing interventions [114, 115], is applied. This is done in order to assist the patient in choosing his most efficient strategy, leading to the patient experiencing improvements in task performance. If done properly, already in the first two weeks of arm-hand training the vast majority of moderately affected patients learn to recognize and to monitor improvements made in AHF and AHSP.

\section{Methodological considerations}

\section{Intention-to-treat procedure}

A study to evaluate the course of the affected arm-hand during and after rehabilitation was performed (dossier number NL35681.068.11). This study was divided in two parts: In the first part, the course of AHF and AHSP capacity has been evaluated [61], and in the second part actual arm-hand use has been evaluated [75]. A single-armed prospective cohort study was conducted in order to collect the necessary data. Based on their level of arm-hand impairment, stroke survivors were stratified into three different subgroups; severely, moderately and mildly impaired before being admitted to a training program fitted to their initial arm-hand impairment level [13]. This was done using the Utrecht Arm-hand Test [14].

Approximately one fourth of patients in the severely affected subgroup (subgroup 1) showed more than expected progressions measured at AHF level, AHSP level and actual arm-hand use level. One could argue that a part of these patients may have been allocated to the wrong subgroup and thereby to the wrong intervention, due to misclassification. However, this was not the case. Up to $30 \%$ of stroke patients cope with a severely impaired arm-hand [89, 90]. This severely affected sub-acute stroke group consists of patients who have roughly a $50 \%$ chance of (some) recovery of function versus no recovery at all [51]. Furthermore, currently used clinical measures may not be able to adequately predict expected recovery in patients with a severely impaired arm-hand [55, 116]. 
In both our studies, all data with respect to AHF, AHSP and actual arm-hand-use in the subgroups was analyzed according to the intention-to-treat principle. All subjects were analyzed according to the subgroups they were assigned to in the first place. The intention-to-treat procedure performed in this study provides an optimal reflection of treatment as provided in daily practice, and retains the initial comparison between the three subgroups [117]. As this could have led to a higher within-group variance and a lower between-group contrast regarding the outcome measures used, in the second study, also a per-protocol analysis of the data was performed, i.e. leaving out patients who, in view of their speed of progress, switched to another program of CARAS, to check if results regarding actual-arm-hand use differed significantly from the results obtained in the intention-to-treat analysis. However, no significant differences in outcomes were found between the intention-to-treat analyses and the per-protocol analyses.

\section{Ceiling effects in patients with a mildly affected hand}

With respect to between-group differences, largest gains in AHF and AHSP were observed in patients with an initially moderately affected hand. However, patients with an initially mildly impaired hand may have improved even further as to their AHF and AHSP which may not have been detected due to ceiling effects in the measures used. In two clinical scales encompassing arm-hand function and arm-hand capacity, i.e. the Fugl-Meyer Motor Assessment (FMA) and the Action Research Arm test (ARAT), the maximum of the scale was reached in some patients, thus constituting ceiling effects [71].

In order to measure the quality of motor performance, core clinical measures like the FMA and ARAT may be complemented by technological devices involving sensor technology. The application of sensor technology to monitor kinematics and kinetics can be used as additional parameters to objectively assessing arm-hand performance and the quality of arm-hand use in stroke trials, without being hampered by floor or ceiling effects [118-120]. Furthermore, kinematic assessments are assumed to provide information about physiologically relevant changes during the neurophysiological recovery episode. In the long term, this could lead to a better understanding of neurological mechanisms of sensorimotor recovery post-stroke [121]. Additionally, the use of multiple sensors may be useful in recognizing arm-hand activities which are 
performed by patients in their home environment [122], and for assessing the quality of arm-hand use in daily tasks, which may be incorporated as important outcome measures in arm-hand assessment procedures [123].

\section{Within-group variations}

In both AMUSE studies large within-group variations across time regarding AHF and AHSP both during and after rehabilitation were observed in all three subgroups. The purpose of the AMUSE study was to evaluate the course of AHF and AHSP in a heterogeneous group of stroke survivors in the sub-acute phase, typically seen in stroke rehabilitation. This entails that a) The study population covered a broad spectrum of hand severity levels (as measured at entry of the study), and contained a large heterogeneity as to recovery patterns of the affected arm-hand; b) Only a few inclusion and exclusion criteria were used, leading to a broad spectrum of patient characteristics, and co-morbidities; and c) As measures were taken at fixed, predetermined time points post-stroke, and patients' individual neurophysiological progressions differed, within-group variance may have increased as time post-stroke progressed. Pragmatically, in the CARAS program, this clinical variation in patients' status has been taken into account by introducing three distinct, yet cohesive training programs, keeping patients' variability clinically manageable.

\section{Measuring self-perceived performance}

Patients may fail to experience the positive impact of their arm-hand improvements due to e.g. problems regarding their mood and social participation, especially in the post-rehabilitation phase $[21,124]$. In a percentage of our study population, the level of self-perceived performance, as measured with the ABILHAND, deteriorated or remained the same over time. This percentage was higher relative to those concerning outcomes on more objectively measured outcomes at the level of AFH and AHSP capacity. The aforementioned mood and social participation levels may play a role here. Another plausible reason may be that the ABILHAND does not cover all aspects of self-perceived performance. For example, a patient may improve on a personally relevant (daily) skill, which is not covered by the ABILHAND. An alternative for the ABILHAND with respect to self-perceived performance may be the DASH (Disabilities of the Arm, Shoulder and Hand) [125]. This questionnaire helps to describe and rate 
the disability experienced by people with upper-limb disorders and also helps to monitor changes in symptoms and function over time.

\section{Single case experimental design versus randomized controlled trial}

In a number of the studies reported in this thesis, a single case experimental design (SCED) has been used to evaluate the added-value of new therapy elements added to CARAS, and to deal with multiple sources of variability in sub-acute stroke patients with a moderately to severely affected arm-hand [126]. Randomized controlled trails (RCTs) are considered the gold standard for evaluating efficacy in clinical research to constitute evidence for medical treatment $[127,128]$. Nevertheless, to translate RCT data stemming from (heterogeneous) group averages into individual patient-specific clinical practice is considered difficult $[127,129,130]$. Given the individual patient's characteristics, clinicians (and also patients) want to know / predict the extent to which (arm-hand) interventions may improve the individual patient's performance. The SCED methodology provides a solution in determining the effectiveness of rehabilitation interventions in the individual patient, which is coherent to personalized evidencebased medicine. However, the generalizability of SCED results towards larger populations is low. The SCED allows training of the individual patient in clinical practice while observing and recording his (behavioral) changes [131]. Observed changes, like for instance improvements in arm-hand capacity, are directly applicable to the patient's situation and thus easily interpretable. Consequently, the application of SCED studies have the potential to make a substantial contribution to the development of evidence based interventions [132].

Some specific threats to the internal validity make the SCED methodologically challenging [126, 132]. Featuring a within-subject design, participants serve as their own controls over the pre- and post-experimental phase. Main outcomes are to be measured systematically and frequently within and across all phases, in order to assess individual change across different conditions. Stability of the dependent variable during baseline conditions, or at least being able to adequately correct for effects of other treatment and effects of spontaneous recovery during baseline, is essential, because otherwise the detection of specific treatment effects will not be feasible [133].

Also, in order to adequately quantify baseline variability, the number of measurements taken during the baseline period should be extensive [134]. However, in some cases 
lengthening the baseline phase is undesirable, especially in conditions where changes in the "natural" course of recovery are to be expected, like in the sub-acute phase after a stroke. Furthermore, extensive repetition of measurements can also have an exhausting and demotivating effect on patients.

\section{Detrending for baseline trends}

In our SCED studies, linear de-trending has been applied for any (baseline) trend for each single subject in order to identify the unique contribution of the applied intervention. Nevertheless, the application of linear detrending may have led in some cases to a) An overestimation of spontaneous recovery and effects of therapy-asusual, especially in the long term post-training, thereby underestimating the effects of the experimental stimulus, i.e. the added-value of the application of a dynamic hand orthosis or botulinum toxin in the follow-up phase. For sub-acute stroke patients, an Sshaped (non-linear) model or at least a model 'levelling off over time' might have better fitted the reality of spontaneous recovery. However, gathering enough data to adequately fit such a model would have necessitated a much longer baseline phase, which, in turn, would inevitably have compromised the sub-acuteness of the patients' status during the ensuing intervention phase. Future research should focus on optimizing this model to better contrast spontaneous recovery with intermediate and long-term effects of (adjunct) therapy. 


\section{Future perspectives}

Apart from answering a number of clinical questions, this thesis has also raised a number of new questions that need attention.

Future rehabilitation of patients with a moderately to severely affected arm-hand A large number of studies on rehabilitation treatment outcomes aimed at the impaired arm-hand reported in literature has and still is focusing on sharply delineated subgroups of stroke patients of homogeneous composition, and have been only limiting taking the wider spectrum of co-morbidities associated with stroke into account [135]. This has led, among others, to less scientific attention for patients with a moderately to severely impaired arm-hand who typically experience high (betweensubject) variances in comorbidity, and, therefore, to less development of novel, efficacious arm-hand training interventions for these subgroups. What is needed is an integrated approach in which clinicians and researchers co-develop new arm-hand training interventions for these subgroups, and novel (statistical) methodologies that accommodate the between-subject versatility typically seen in (daily) clinical stroke rehabilitation practice in general, and in moderately to severely impaired stroke patient specifically. Such an approach would necessitate the set-up of an academic specialty, or network, thus facilitating the interaction of experts in different fields of rehabilitation, methodology, movement/health sciences, and technology.

Early identification of recovery potential in patients with a severely affected arm-hand The identification of severely impaired patients' eligibility to motor recovery at an early stage post-stroke is important for tailoring neuro-rehabilitation interventions. I.e., it is essential to be able to differentiate between severely impaired individuals, who have no prospect on arm-hand recovery, and those that may attain partial or even complete recovery of arm function. Current prediction models need optimisation as to this feature. There is a growing interest in using biomarkers to predict patients' motor recovery at AHF and AHSP level in severely affected patients. Further research into supporting biomarker-based arm-hand outcome prediction modeling is necessary. 
Appropriate allocation of patients to dedicated technology-assisted arm-hand rehabilitation In order to optimally exploit both training-induced and endogenous post-stroke neuroplasticity, typically seen in a limited time window, an early start of treatment is essential, even when no voluntary activity of the hand muscles has been observed yet. However, to participate in regular arm-hand skill training at the start of rehabilitation is difficult for these patients due to their insufficient ability to voluntarily generate force. The application of technological assistive devices, like, for instance, actuated dynamic hand-orthoses, may assist them in performing grasp and release movements independently. Once equipped with an actuated dynamic orthosis, they may engage in CARAS' gross motor grip training interventions early. Working actively with their nonfunctional arm-hand, using an actuated dynamic hand orthosis, may provide meaningful tactile and proprioceptive feedback associated with motor commands, enhancing sensorimotor learning. Patients with a severely affected hand who, potentially due to more precise biomarker-based recovery models, are properly admitted to a well-defined, task-oriented training method, equipped with assistive technology devices, may obtain substantial improvements in terms of AHF, AHSP capacity and actual arm-hand-use. Future research should focus on investigating the efficacy of such newly developed care pathways and training interventions. In order to contrast these (novel) interventions, the longitudinal database on the development of AHF and AHSP, as outlined in this thesis, can be used as a reference database.

Synergistic use of a task-oriented approach and technology in arm-hand treatment Given the sheer volume of stroke patients experiencing arm-hand problems and the decreasing (financial) means $[136,137]$ to provide adequate treatment to them, new solutions must be sought to maintain, optimize and improve treatment outcome. The use of technology as part of regular treatment definitively is a solution here. However, there are several challenges, ranging from "What works for whom?" and "How to adequately incorporate technology in assisting treatment?" to "How (cost-)effective is the use of technology incorporated in (daily) rehabilitation treatment?". It is advocated to start investigating how low-cost, non-actuated technology can be used in mildly affected stroke patients to free up therapist-assisted treatment time that can then be spent on patients with a moderately to severely affected arm-hand. Furthermore, technology-assistance for the latter groups to be developed, may also lead to further 
improvement of AHF and AHSP. Here, identifying/predicting, at an early stage poststroke, which kind of technology-assistance, e.g. robot-based or sensor-based technology, may work best for which kind of arm-hand severity subgroup is of paramount importance. For an adequate implementation of technology in regular treatment, implementation strategies should be developed. Also, in order to assess the (cost-)effectiveness of newly developed technology-assisted treatment regimes, health technology assessment studies should be performed. 


\section{References}

1. Lang C, Lohse K, Birkenmeier R. Dose and timing in neurorehabilitation: Prescribing motor therapy after stroke. Current opinion in neurology. 2015;28(6):549-55. doi: 10.1097/WCO.0000000000000256.

2. Demetrios M, Gorelik A, Louie J, Brand C, Baquley IJ, Khan F. Outcomes of ambulantory rehabilitation programmes following botulinum toxin for spasticity in adults with stroke. J Rehabil Med. 2014;46:730-7.

3. Bernhardt J, Borschmann K, Boyd L. Moving rehabilitation research forward: developing consensus statements for rehabilitation and recovery research. International Journal of Stroke. 2016;11:454-8.

4. Ballinger C, Ashburn A, Low J, Roderick P. Unpacking the black box of therapy - a pilot study to describe occupational therapy and physiotherapy interventions for people with stroke. Clinical Rehabilitation. 1999;13:301-9.

5. DeJong G, Horn SD, Gassaway JA, Slavin MD, Dijkers MP. Toward a taxonomy of rehabilitation interventions: Using an inductive approach to examine the "black box" of rehabilitation. Archives of Physical Medical Rehabililitation. 2004;85(4):678-86. PubMed PMID: 15083447.

6. Demetrios M, Brand C, Louie J, Khan F. More than a Black Box of Rehabilitation: Characterizing therapy programmes following Botulinum Toxin Injections for Spasticity in Adults with Stroke Journal of Rehabilitation Medicine. 2016;48:426-34.

7. Pollock A, Farmer SE, Brady MC, Langhorne P, Mead GE, Mehrholz J, et al. Interventions for improving upper limb function after stroke. The Cochrane database of systematic reviews. 2014;11:CD010820. Epub 2014/11/12. doi: 10.1002/14651858.CD010820.pub2. PubMed PMID: 25387001.

8. Veerbeek JM, van Wegen E, van Peppen R, van der Wees PJ, Hendriks E, Rietberg M, et al. What is the evidence for physical therapy poststroke? A systematic review and metaanalysis. PloS One. 2014;9(2):e87987. Epub 2014/02/08. doi: 10.1371/journal.pone.0087987. PubMed PMID: 24505342; PubMed Central PMCID: PMCPMC3913786.

9. DeJong G, Horn SD, Conroy B, Nichols D, Healton EB. Opening the black box of poststroke rehabilitation: stroke rehabilitation patients, processes, and outcomes. Archives of physical medicine and rehabilitation. 2005;86(12 Suppl 2):S1-S7. PubMed PMID: 16373135.

10. Richards LG, Latham NK, Jette DU, Rosenberg L, Smout RJ, DeJong G. Characterizing occupational therapy practice in stroke rehabilitation. Archives of physical medicine and rehabilitation. 2005;86(12 Suppl 2):S51-S60. PubMed PMID: 16373140. 
11. Bode K, Heinemann A, Semik P, Mallinson T. Patterns of therapy activities across length of stay and impairment levels: Peering inside the "black box" of inpatient stroke rehabilitation. Archives of Physical Medical Rehabililitation. 2004;85:1901-8.

12. Brewer L, Horgan F, Hickey A, Williams D. Stroke rehabilitation: recent advances and future therapies. An International Journal of Medicine. 2013;106(1):11-25. doi: 10.1093/qjmed/hcs174.

13. Franck JA, Halfens JAM, Smeets RJEM, Seelen HAM. Concise Arm and hand Rehabilitation Approach in Stroke (CARAS): A practical and evidence-based framework for clinical rehabilitation management. The Open Journal of Occupational Therapy. 2015;3(4):Article 10. doi: 10.15453/2168-6408.1164.

14. Kruitwagen-van Reenen ET, Post MW, Mulder-Bouwens K, Visser-Meily JM. A simple bedside test for upper extremity impairment after stroke: validation of the Utrecht Arm/Hand Test. Disability and rehabilitation. 2009;31(16):1338-43.

15. Brunnstrom $\mathrm{S}$. Motor testing procedures in hemiplegia: based on sequential recovery stages. Physical therapy. 1966;46:357 - 75.

16. Kwakkel G, Kollen B, Lindeman E. Understanding the pattern of functional recovery after stroke: facts and theories. Restor Neurol Neurosci. 2004;22(3-5):281-99. PubMed PMID: 15502272.

17. Winstein C, Lewthwaite R, Blanton S, Wolf L, Wishart L. Infusing Motor Learning ResearchInto Neurorehabilitation Practice: A Historical Perspective With Case Exemplar From the Accelerated Skill Acquisition Program. JNPT. 2014;38:190-200.

18. Arocha J, Patel V, Patel Y. Hypothesis generation and the coordination of theory and evidence in novice diagnostic reasoning. Medical Decision Making. 1993;13(3):198-211. doi: 10.1177/0272989X9301300305.

19. Elstein A, Shulman L, Sprafka S. Medical problem solving: An analysis of clinical reasoning. Cambridge, MA: Harvard University Press; 1978.

20. Shumway-Cook A, Woollacott M. Motor learning and recovery of function. In: Motor control Translating research into clinical practice. Philadelphia: Lippincott Williams \& Wilkins; 2007. p. 21-45.

21. Jones F, Rianzi A. Self-efficacy and self-management after stroke: a systematic review. Disability and Rehabililation. 2011;33(10).

22. Bandura A. Self-efficacy. Ramachaudran V, editor. New York: Academic Press (Reprinted in H. Friedman [Ed.]; 1994.

23. Kristensen H, Persson D, Nygren C, Boll M, Matzen P. Evaluation of evidence within occupational therapy in stroke rehabilitation. Scandinavian Journal of Occupational Therapy. 2011;18:11-25. 
24. Dashtipour K, Chen J, Walker H, Lee M. Systematic Literature Review of AbobotulinumtoxinA in Clinical Trials for Adult Upper Limb Spasticity. American Journal of Physical Medicine \& Rehabilitation. 2015;94(3):229 - 38. doi: 10.1097/PHM.0000000000000208.

25. Saposnik G, Levin M. Virtual reality in stroke rehabilitation: a meta-analysis and implications for clinicians. Stroke; a journal of cerebral circulation. 2011;42(5):1380-6. Epub 2011/04/09. doi: 10.1161/strokeaha.110.605451. PubMed PMID: 21474804.

26. Coscia M, Wessel M, Chaudary U, del R Millán J, Micera S, Guggisberg A, et al. Neurotechnology-aided interventions for upper limb motor rehabilitation in severe chronic stroke. Brain. 2019;142(8):2182-97. doi: doi.org/10.1093/brain/awz181.

27. Quandt F, Hummel F. The influence of functional electrical stimulation on hand motor recovery in stroke patients: a review. Experimental \& Translational Stroke Medicine. 2014;6(9):1-7.

28. Alon G. Functional Electrical Stimulation (FES): Transforming Clinical Trails to NeuroRehabilitation Clinical Practice - A forward perspective. J Nov physiother 2013;3(5):1-9.

29. Mehrholz J, Pohl M, Platz T, Kugler J, Elsner B. Electromechanical and robot-assisted armtraining for improving activities of daily living, armfunction, and arm muscle strength after stroke (Review). Cochrane Database of Systematic Reviews. 2018;(9). doi: 10.1002/14651858.CD006876.pub5.

30. Gassert R, Dietz V. Rehabilitation robots for the treatment of sensorimotor deficits: a neurophysiological perspective. Journal of neuroengineering and rehabilitation. 2018;15(46):1 - 15. doi: 10.1186/s12984-018-0383-x.

31. Bos R, Haarman C, Stortelder T, Nizamis K, Herder J, Stienen A, et al. A structured overview of trends and technologies used in dynamic hand orthoses. Journal of neuroengineering and rehabilitation. 2016;13(62):2 - 25.

32. Franck JA, Smeets RJEM, Seelen HAM. Evaluation of a functional hand orthosis combined with electrical stimulation adjunct to arm-hand rehabilitation in subacute stroke patients with a severely to moderately affected hand function. Disability and rehabilitation. 2018:1-9

33. Franck JA, Timmermans AA, Seelen HAM. Effects of a dynamic hand orthosis for functional use of the impaired upper limb in sub-acute stroke patients: A multiple single case experimental design study. Technology and Disability. 2013;25:177-87.

34. Carpaneto J, Micera S. Application of orthoses and neurostimulation in neurorehabilitation in: Oxford book of Neurorehabilitation. In: Christopher K, editor. First Edition ed. Oxford, United Kingdom: Oxford University Press; 2015. p. 263-73.

35. Berwick D. Dissiminating Innovations in Health Care. Journal of American Medical Association. 2003;289(15):1969 - 75. 
36. Langan J, Subryan H, Nwogu I, Cavuoto L. Reported use of technology in stroke rehabilitation by physical and occupational therapists. Disability Rehabilitation Assistive Technology. 2018;13(7):641 - 7. doi: 10.1080/17483107.2017.

37. Burridge $\mathrm{JH}$, Hughes AM. Potential for new technologies in clinical practice. Current opinion in neurology. 2010;23(6):671-7. PubMed PMID: 20962639.

38. Bhakta B. Management of spasticity in stroke. Br Med Bull. 2000;56(2):476-85.

39. Royal College of Physicians BSoRM, The Chartered Society of Physiotherapy, Association of Chartered Physiotherapists in Neurology and the Royal College of Occupational Therapists. Spasticty in adults: management of botulinum toxin national guidelines 2018 London: RCP; 2018.

40. Kinnear B, Lannin N, Cusick A, Harvey L, Rawicki B. Rehabilitation Therapies After Botulinum Toxin-A Injection to Manage Limb Spasticity: a Systematic Review. Physical Therapy. 2014;94:1569-81.

41. Devier D, Harnar J, Lopez L, Brashear A, Graham G. Rehabilitation plus OnabotulinumtoxinA Improves Motor Function over OnabotulinumtoxinA Alone in PostStroke Upper Limb Spasticity: A Single-Blind Randomized Trail. Toxins. 2017;9(216):1-9.

42. Andringa A, Port I, van Wegen E, Ket J, Meskers C, Kwakkel G. Effectiveness of Botulinum Toxin Treatment for Upper Limb Spasticity Poststroke Over Different ICF Domains: a Systematic Review and Meta-Analysis. Archives of physical medicine and rehabilitation. 2019. doi: doi.org/10.1016/j.apmr.2019.01.016.

43. Teasell R, Mehta S, Pereira S, Mclntyre A, Janzen S, Allen L, et al. Time to rethink longterm rehabilitation management of stroke patients. Top Stroke Rehabilitation 2012;19(6):457 - 62. doi: 10.1310/tsr1906-457.

44. Demain S, Burridge J, Ellis-Hill C, Hughes A, Yardley L, Tedesco-Triccas L, et al. Assistive technologies after stroke: self-management or fending for yourself? A focus group study. BMC health services research. 2013;13:1 - 12.

45. Hughes AM, Burridge JH, Demain SH, Ellis-Hill C, Meagher C, Tedesco-Triccas L, et al. Translation of evidence-based Assistive Technologies into stroke rehabilitation: users' perceptions of the barriers and opportunities. BMC health services research. 2014;14:124. Epub 2014/03/14. doi: 10.1186/1472-6963-14-124. PubMed PMID: 24620739; PubMed Central PMCID: PMCPMC4007558.

46. Veerbeek J, Langbroek-Amersfoort C, van Wegen E, Meskers C, Kwakkel G. Effects of Robot-Assisted Therapy for the Upper Limb After Stroke: A Systematic Review and Metaanalysis. Neurorehabilitation and neural repair. 2017;31(2):107 - 212. doi: $10.1177 / 1545968316666957$.

47. Herzlinger RE. Why innovation in health care is so hard. Harv Bud Rev. 2006;84(5):5866. 
48. Pham Q, Wiljer D, Cafazzo J, PEng P. Beyond the Randomized Controlled Trial: A Review of Alternatives in mHealth Clinical Trial Methods JMIR mHealth uHealth. 2016;4(3):1-20.

49. Kumar S, Nilsen W, Abernethy A, Atienza A, Patrick K, Pavel M, et al. Mobile health technology evaluation: the mHealth evidence workshop. American Journal of Prevention and Medicine. 2013;45(2):228-36.

50. Taub E, Uswatte G. Constraint-Induced Movement therapy: answers and questions after two decades of research. NeuroRehabilitation. 2006;21(2):93-5. PubMed PMID: 16917156.

51. Winters C, Kwakkel G, Nijland R, van Wegen E. When Does Return of Voluntary Finger Extension Occur Post-Stroke? A Prospective Cohort Study. PloS one. 2016;11(8):1-12. doi: 10.1371/journal.pone.0160528.

52. Prabhakaran S, Zarahn E, Riley C, Speizer A, Chong JY, Lazar RM, et al. Inter-individual variability in the capacity for motor recovery after ischemic stroke. Neurorehabilitation and neural repair. 2008;22(1):64-71. Epub 2007/08/10. doi: 10.1177/1545968307305302. PubMed PMID: 17687024.

53. Winters C, van Wegen E, Daffertshofer A, Kwakkel G. Generalizability of the Proportional Recovery Model for the Upper Extremity After an Ischemic Stroke. Neurorehabilitation and neural repair. 2015;29(7):614-22. Epub 2014/12/17. doi: 10.1177/1545968314562115. PubMed PMID: 25505223.

54. Buch E, Rizk S, Nicolo P, Cohen L, Schnider A, Guggisberg A. Predicting motor improvement after stroke with clinical assessment and diffusion tensor imaging. Neurology. 2016;86:1924 - 5.

55. Byblow W, Stinear C, Barber P, Petoe M, Ackerley S. Proportional Recovery After Stroke Depends on Corticomotor Integrity. ANN NEUROL 2015;78:848-59.

56. Krakauer J, Cortés J. A non-task-oriented approach based on high-dose playful movement exploration for rehabilitation of the upper limb early after stroke: A proposal. NeuroRehabilitation. 2018 43:31-40. doi: 10.3233/NRE-172411.

57. Daly J, McCabe J, Holcomb H, Monkiewicz M, Gansen J, Pundik S. Long-Dose Intensive Therapy Is Necessary for Strong, Clinically Significant, Upper Limb Functional Gains and Retained Gains in Severe/Moderate Chronic Stroke. Neurorehabilitation and neural repair. 2019. doi: $10.1177 / 1545968319846120$.

58. Bell A, Wolke M, Ortez R, Jones $T$, Kerr A. Training intensity impacts motor rehabilitation efficacy following unilateral ischemic insult of the sensorimotor cortex in C57BL/6 mice. Neurorehabilitation and neural repair. 2014;29(6):590-8. doi: 10.1177/1545968314553031 . 
59. Ward N, Brander F, Kelly K. Intensive upper limb neurorehabilitation in chronic stroke: outcomes from the Queen Square programme. cerebrovasc Dis. 2019;90:498-506. doi: 10.1136/jnnp-2018-319954.

60. Kwakkel G, van Peppen R, Wagenaar RC, Wood Dauphinee S, Richards C, Ashburn A, et al. Effects of augmented exercise therapy time after stroke: a meta-analysis. Stroke; a journal of cerebral circulation. 2004;35(11):2529-39. PubMed PMID: 15472114.

61. Franck JA, Smeets RJEM, Seelen H. Changes in arm-hand function and arm-hand skill performance in patients after stroke during and after rehabilitation. PloS one. 2017;12(6):1 $-18$.

62. Winstein CJ, Stein J, Arena R, Bates B, Cherney LR, Cramer SC, et al. Guidelines for Adult Stroke Rehabilitation and Recovery A Guideline for Healthcare Professionals From the American Heart Association/American Stroke Association. Stroke; a journal of cerebral circulation. 2016;47:0000.

63. Hunter S, Johansen-Berg H, Ward N, Kennedy N, Chandler E, Weir C, et al. Functional strength Training and Movement Performance Therapy

for Upper limb recovery early Poststroke efficacy, neural correlates, Predictive Markers, and cost-effectiveness: FasT-indicaTe Trial. Frontiers in Neurology. 2018;8(733):1 - 24. doi: 10.3389/fneur.2017.00733.

64. Combs SA, Kelly SP, Barton R, Ivaska M, Nowak K. Effects of an intensive, task-specific rehabilitation program for individuals with chronic stroke: a case series. Disability and rehabilitation. 2010;32(8):669-78. PubMed PMID: 20205581.

65. Bosch J, O'Donnell M, Barreca S, Thabane L, Wishart L. Does Task-Oriented Practice Improve Upper Extremity Motor Recovery after Stroke? A Systematic Review. ISRN Stroke. 2014:1 - 10. doi: 10.1155/2014/504910.

66. Waddel K, Strube M, Bailey R, Klaesner J, Birkenmeier R, Dromerick A, et al. Does taskspecific training improve upper limb performance in daily life post-stroke? Neurorehabilitation and neural repair. 2017;31(3):290 - 300.

67. Rijntjes M, Haevernick K, Barzel A, van den Bussche H, Ketels G, Weiller C. Repeat therapy for chronic motor stroke: a pilot study for feasibility and efficacy. Neurorehabilitation and neural repair. 2009;23(3):275-80.

68. Kwakkel G, Kollen BJ, Wagenaar RC. Long term effects of intensity of upper and lower limb training after stroke: a randomised trial. J Neurol Neurosurg Psychiatry. 2002;72(4):473-9. PubMed PMID: 11909906.

69. Uswatte G, Taub E, Bowman M, Delgado A, Bryson C, Morris D, et al. Rehabilitation of stroke patients with plegic hands: Randomized controlled trial of expanded ConstraintInduced Movement therapy. Restorative Neurology \& Neuroscience 2018;36(2 ):225 - 44. doi: 10.3233/RNN-170792. 
70. Meyer S, Verheyden G, Brinkmann N, Dejaeger E, De Weerdt W, Feys H, et al. Functional and motor outcome 5 years after stroke is equivalent to outcome at 2 months: follow-up of the collaborative evaluation of rehabilitation in stroke across Europe. Stroke 2015;46(6):1613 -9. doi: 10.1161/STROKEAHA.115.009421.

71. Gladstone DJ, Danells CJ, Black SE. The fugl-meyer assessment of motor recovery after stroke: a critical review of its measurement properties. Neurorehabilitation and neural repair. 2002;16(3):232-40. PubMed PMID: 12234086.

72. Hamilton GF, McDonald C, Chenier T. Meting van de grijpkracht: waarde en betrouwbaarheid van de bloeddrukmeter en de 'Jamar grijpdynamometer'. Stimulus. 1994;Volume 13(3):164-5.

73. Lyle RC. A performance test for assessment of upper limb function in physical rehabilitation treatment and research. Int J Rehabil Res. 1981;4(4):483-92. PubMed PMID: 7333761.

74. Penta M, Thonnard JL, Tesio L. ABILHAND: a Rasch-built measure of manual ability. Archives of physical medicine and rehabilitation. 1998;79(9):1038-42. PubMed PMID: 9749680.

75. Franck JA, Smeets RJEM, Seelen HAM. Changes in actual arm-hand use in stroke patients during and after clinical rehabilitation involving a well-defined arm-hand rehabilitation program: A prospective cohort study. PloS one. 2019:1-22. doi: http://doi.org/10.1371/journal.pone.0214651

76. Hayward K, Kuys S, Barker R, Brauer S. Can stroke survivors with severe upper arm disability achieve a clinically important change in arm function during inpatient rehabilitation? A multicentre, prospective, observational study. NeuroRehabilitation. 2014;35(1):17-23. Epub 2014/07/06. doi: 10.3233/nre-141096. PubMed PMID: 24990005.

77. Korpershoek C, van der Bij J, Hafsteinsdóttir T. Self-efficacy and its influence on recovery of patients with stroke: a systematic review. Journal of Advanced Nursing. 2011;67(9):1876-94. doi: 10.1111/j.1365-2648.

78. Maujean A, Davis P. The Relationship between Self-Efficacy and Well-Being in Stroke Survivors. International Journal of Physical Medicine \& Rehabilitation. 2013;1(7). doi: 10.4172/2329-9096.1000159.

79. Kong K, Loh Y, Thia E, Chai E, Yin C. The Relationship between Poststroke Depression and Upper Limb Recovery in Patients Admitted to a Rehabilitation Unit. Physical medicine and rehabilitation. 2017;1(1):1-7.

80. Rand D, Eng J. Predicting Daily Use of the Affected Upper Extremity 1 Year after Stroke. Journal of Stroke and Cerebrovascular Diseases. 2015;24(2):274 - 283.

81. Persson HC, Opheim A, Lundgren-Nilsson Å, Alt Murphy M, Danielsson A, Sunnerhagen KS. Upper extremity recovery after ischaemic and haemorrhagic stroke: Part of the 
SALGOT study. European Stroke Journal. 2016;1(4):310 - 9 . doi: 10.1177/2396987316672809.

82. Hendricks HT, van Limbeek J, Geurts AC, Zwarts MJ. Motor recovery after stroke: a systematic review of the literature. Archives of physical medicine and rehabilitation. 2002;83(11):1629-37. PubMed PMID: 12422337.

83. Broeks JG, Lankhorst GJ, Rumping K, Prevo AJ. The long-term outcome of arm function after stroke: results of a follow-up study. Disability and rehabilitation. 1999;21(8):357-64. PubMed PMID: 10503976.

84. Uswatte G, Foo WL, Olmstead H, Lopez K, Holand A, Simms LB. Ambulatory monitoring of arm movement using accelerometry: an objective measure of upper-extremity rehabilitation in persons with chronic stroke. Archives of physical medicine and rehabilitation. 2005;86(7):1498-501. PubMed PMID: 16003690.

85. Arya K, Verma R, Garg R, Sharma V, Agarwal M, Aggarwal G. Meaningful Task-Specific Training (MTST) for Stroke Rehabilitation: A Randomized Controlled Trial. Topics in Stroke Rehabilitation 2012;19(3):193 - 211. doi: 10.1310/tsr1903-193.

86. Doman C, Waddell K, Bailey R, Moore J, Lang C. Changes in Upper-Extremity Functional Capacity andDaily Performance During Outpatient Occupational Therapy for People With Stroke. The American Journal of Occupational Therapy. 2016;70.

87. Taub E, Uswatte G, Mark VW, Morris DM. The learned nonuse phenomenon: implications for rehabilitation. Eura Medicophys. 2006;42(3):241-56. PubMed PMID: 17039223.

88. Esquenazi A, Mayer N. Botulinum toxin for the management of muscle overactivity and spasticity after stroke. Current atherosclerosis reports. 2004;3:295-8.

89. Nakayama H, Jorgensen HS, Raaschou HO, Olsen TS. Recovery of upper extremity function in stroke patients: the Copenhagen Stroke Study. Archives of physical medicine and rehabilitation. 1994;75(4):394-8. Epub 1994/04/01. PubMed PMID: 8172497.

90. Kwakkel G, Kollen BJ, van der Grond J, Prevo AJ. Probability of regaining dexterity in the flaccid upper limb: impact of severity of paresis and time since onset in acute stroke. Stroke; a journal of cerebral circulation. 2003;34(9):2181-6. PubMed PMID: 12907818.

91. Nijland RH, van Wegen EE, Harmeling-van der Wel BC, Kwakkel G. Presence of finger extension and shoulder abduction within 72 hours after stroke predicts functional recovery: early prediction of functional outcome after stroke: the EPOS cohort study. Stroke; a journal of cerebral circulation. 2010;41(4):745-50. Epub 2010/02/20. doi: 10.1161/strokeaha.109.572065. PubMed PMID: 20167916.

92. Langhorne P, Bernhardt J, Kwakkel G. Stroke rehabilitation. Lancet. 2011;377:1693 - 702.

93. Van Bragt P, van Ginekken BT, Westendorp T, Heijenbrok-Kal MJ, Wijffels MP, Ribbers GM. Predicting outcome in a post acute stroke rehabilitation programme. International Journal of Rehabilitation Research. 2014;37:110 - 7. 
94. Hatem SM, Saussez G, Faille M, Prist V, Zhang X, Dispa D, et al. Rehabilitation of Motor Function after Stroke: A Multiple Systematic Review Focused on Techniques to Stimulate Upper Extremity Recovery. Frontiers in human neuroscience. 2016;10.

95. Barreca S, Wolf SL, Fasoli S, Bohannon R. Treatment interventions for the paretic upper limb of stroke survivors: a critical review. Neurorehabilitation and neural repair. 2003;17(4):220-6. PubMed PMID: 14677218.

96. Teasell RW, Kalra L. What's new in stroke rehabilitation: back to basics. Stroke; a journal of cerebral circulation. 2005;36(2):215-7. PubMed PMID: 15637318.

97. Canning CG, Ada L, Adams R, O'Dwyer NJ. loss of strength contributes more to physical disability after stroke than loss of dexterity. Clinical rehabilitation. 2004;18(3):300 - 8.

98. Taub E, Miller NE, Novack TA, Cook EW 3rd, Fleming WC, Nepomuceno CS, et al. Technique to improve chronic motor deficit after stroke. Archives of physical medicine and rehabilitation.1993;74(4):347-54. Epub 1993/04/01. PubMed PMID: 8466415.

99. Winters C, Kwakkel G, van Wegen E, Nijland R, Veerbeek J, Meskers C. Moving stroke rehabilitation forward: The need to change research. NeuroRehabilitation. 2018;43:19-30. doi: 10.3233/NRE-172393.

100.Kwah L, Herbert R. Prediction of Walking and Arm Recovery after Stroke: A Critical Review. Brain Sciences. 2016;6(53):1-16.

101. Hoonhorst MH, Nijland RH, van den Berg J, Emmelot CH, Kollen BJ, Kwakkel G. How Do Fugl-Meyer Arm Motor Scores Relate to Dexterity According to the Action Research Arm Test at 6 Months Poststroke. Archives of physical medicine and rehabilitation. 2015;96:1845 - 9.

102.Woytowicz E, Rietschel J, Goodman RN, Conroy SS, Sorkin JD, Whitall J, et al. Determining Levels of Upper Extremity Movement Impairment by Applying Cluster Analysis to Upper Extremity Fugl-Meyer Assessment in Chronic Stroke. Archives of Physical Medical Rehabilitation 2017;98(3):456 - 62. doi: 10.1016/j.apmr.2016.06.023.

103.Woodbury ML, Velozo CA, Richards LG, Duncan PW. Rasch analysis staging methodology to classify upper extremity movement impairment after stroke. Archives of Physical Medical Rehabilitation. 2013;94(8):1527 - 33.

104.van Wijck FM, Pandyan AD, Johnson GR, Barnes MP. Assessing Motor Deficits in Neurological Rehabilitation: Patterns of Instrument Usage. Neurorehabilitation and neural repair. 2001;15(1):23 - 30.

105.Velstra IM, Ballert CS, Cieza A. A systematic literature review of outcome measures for upper extremity function using the international classification of functioning, disability, and health as reference. PM R. 2011; 3(9):846-860.

106. Hayward KS, Schmidt J, Lohse KR, Peters S, Bernhardt J, Lannin N, et al. Are we armed with the right data? Pooled individual data review of biomarkers in people with severe 
upper limb impairment after stroke. Neuroimage Clinical. 2017;26(15):53 - 5. doi: 10.1016/j.nicl.2016.09.015.

107.Stinear CM, Byblow WD, Ackerley SJ, Smith MC, Borges VM, Barber A. PREP2: A biomarker-based algorithm for predicting upper limb function after stroke. Annals of Clinical and Translational Neurology. 2017;4(11):811 - 20. doi: 10.1002/acn3.488.

108. Hope TM, Friston K, Price C, Leff A, Rotshtein P, Bowman H. Recovery after stroke: not so proportional after all? . Brain. 2019;142(1):15 - 22. doi: 10.1093/brain/awy302.

109. Hayward KS, Lohse KR, Bernhardt, Lang CE, Boyd L. Characterising Arm Recovery in People with Severe Stroke (CARPSS): protocol for a 12-month observational study of clinical, neuroimaging and neurophysiological biomarkers. BMJ Open. 2018;8. doi: 10.1136/bmjopen-2018-026435.

110.Mayo NE, Wood-Dauphinee, Cote R, Durcan L, Carlton J. Activity, participation, and quality of life 6 months poststroke. Archives of physical medicine and rehabilitation. 2002;83(8):1035-42.

111.Sterr A, Freivogel S, Schmalohr D. Neurobehavioral aspects of recovery: Assessment of the learned nonuse phenomenon in hemiparetic adolescent. Archives of Physical and Medical Rehabilitation. 2002;83(12):1726-31.

112.Stewart J, Cramer SC. Patient-reported measures provide unique insights into motor function after stroke. Stroke; a journal of cerebral circulation. 2013;44(4):1111 - 6.

113.Taub E, Uswatte G. Constraint-Induced Movement Therapy: A Family of Neurorehabilitation Treatments that Harnesses the Plasticity of the Central Nervous System. Neurol Rehabil 2013;19(3):161-75.

114.Chen S. The influence of self-efficacy on recovery of spontaneous arm use in hemiparetic stroke. University of Southern California: California-DI; 2011.

115.Jones F, Mandy A, Partridge C. Changing self-efficacy in individuals following a first time stroke: preliminary study of a novel self-management intervention. Clinical rehabilitation. 2009;23:522-33.

116.Feng W, Wang J, Landsittel D. Corticospinal tract lesion load-an imaging biomarker for stroke motor outcomes. Annals of Neurology. 2015;78(6):860 - 70 . doi: 10.1002/ana.24510.

117. Yelland L, Sullivan T, Voysey M, Lee K, Cook J, Forbes A. Applying the intention-to-treat principle in practice: Guidance on handling randomisation errors. Clinical Trails. 2015;12(4):418-23.

118.Nordin N, Xie S, Wunsche B. Assessment of movement quality in robot- assisted upper limb rehabilitation after stroke: a review. Journal of neuroengineering and rehabilitation. 2014;11(137). 
119.Santisteban L, Teremetz M, Bleton J, Baron J, Maier M, Lindberg P. Upper Limb outcome measures used in stroke rehabilitation studies: a systematic literature review. Plos one. 2016;11(5). doi: 10.1371/journal.pone.0154792.

120.Thrane G, Emaus N, Askim T, Audney A. Arm use in patients with subacute stroke monitored by accelerometry: association with motor impairment and influence on selfdependence. J Rehabil Med. 2011;43:299-304.

121.Schwarz A, Kanzler C, Lambercy O, Luft A, Veerbeek J. Systematic Review on Kinematic Assessments of Upper Limb Movements After Stroke. Stroke; a journal of cerebral circulation. 2019;50:718 - 27. doi: 10.1161/STROKEAHA.118.023531.

122.Lemmens JM, Timmermans AA, Janssen-Potten YJM, Pulles S, Bakx WGM, Smeets RJEM, et al. recognizing complex upper extremity activities using body worn sensors. PloS one. $2015 ; 10(3)$.

123.Lemmens JM, Timmermans AA, Janssen-Potten YJM, Pulles SA, Geers RPJ, Bakx WGM, et al. Accelerometry measuring the outcome of robot-supported upper limb training in chronic stroke: a randomized controlled trail. PloS one. 2014. doi: 10.1371/journal.pone.0096414.

124.Kendall E, Catalanoa T, Kuipers P, Posnerb N, Buysa N, Charker J. Recovery following stroke: The role of self-management education. Social Science \& Medicine 2007;64:735 - 46.

125. Hudak PL, Amadio PC, Bombardier C. Development of an upper extremity outcome measure: the DASH (disabilities of the arm, shoulder and hand). The Upper Extremity Collaborative Group (UECG). American Journal of Industrial Medicine. 1996;29(6):602-8.

126.Barlow D, Nock MK, Hersen M. Single case experimental designs: strategies for studying behavior change. Cambridge: Pearson Publishing; 2008.

127.Reith C, Landray M, Devereaux PJ. Randomized clinical trials - removing unnecessary obstacles. New England Journal of Medicine. 2013;369:1061-5.

128.Spieth PM, Kubasch AS, Penzlin AI, Illigens BM, Barlinn K, Siepmann T. Randomized controlled trials - a matter of design. Neuropsychiatric Disease and Treatment. 2016;12 1341-9. doi: 10.2147/NDT.S101938.

129.Wunsch H, Linde-Zwirble WT, Angus DC. Methods to adjust for bias and confounding in critical care health services research involving observational data. Critical Care Journal. 2006;21:1 - 7 .

130. Henegan C, Goldacre B, Mahtani KR. Why clinical trial outcomes fail to translate into benefits for patients. Trails. 2017;12(122):1 - 7. doi: 10.1186/s13063-017-1870-2.

131.Radek KS. Single case designs in psychology practice. Health Psychology Research. 2014;2(1551):98 - 9.

132.Tate R, Perdices M, Rosenkoetter U, Wakim D, Godbee K, Togher L, et al. Revision of a method quality rating scale for single-case experimental designs and n-of-1 trials: the 15- 
item Risk of Bias in N-of-1 Trials (RoBiNT) Scale. Neuropsychology and Rehabilitation. 2013;23(5):619 - 38. doi: 10.1080/09602011.

133.Dallery J, Raiff B. Optimizing behavioral health interventions with single-case designs: from development to dissemination. Translational Behavioral Magazine. 2014;4:290-303. doi: 10.1007/s13142-014-0258-z.

134.Tate R, Perdices M, McDonald S, Togher L, Rosenkoetter U. The design, conduct and report of single-case research: Resources to improve the quality of the neurorehabilitation literature. Neuropsychology and Rehabilitation. 2014;24(3-4):315 - 31 . doi: 10.1080/09602011.

135.Nelson M, McKellar KA, Linda LY, Sarah K, Cott MC, Hall R, et al. Stroke rehabilitation evidence and comorbidity: a systematic scoping review of randomized controlled trials. Topics in stroke rehabilitation. 2017;24(5):374 - 80 . doi: 10.1080/10749357.2017.1282412.

136.O'Connor R. The economic benefits of rehabilitation for neurological conditions. In: Ward N, Dietz V, editors. Oxford Textbook of Neurorehabilitation. 1. Oxford: Oxford University Express; 2015.

137.Lui SK, Nguyen MH. Elderly Stroke Rehabilitation: Overcoming the Complications and Its Associated Challenges. Current Gerontology Geriatrics Research. 2018. doi: 10.1155/2018/9853837. 



\title{
Chapter
}

\author{
Valorization
}



In stroke, the loss of the ability to move the arm and hand is a very common, and often persistent and disabling symptom [1]. Stroke patients who have a prospect on recovery of arm-hand function should be facilitated in creating an optimal retention of how to use the full potential of their affected hand in their own daily life pursuits. Organizing and structuring clinical treatment supporting stroke patients with a moderately or severely affected hand in regaining an optimal level of arm-hand function and armhand skill performance is difficult.

This thesis provides a perspective on how to clinically manage sub-acute stroke patients with a moderately to severely affected arm-hand, regularly seen in stroke rehabilitation settings.

The findings of this thesis may contribute to an increase in quality, efficacy and efficiency of treatment from both the perspective of health care professionals and patients. Furthermore, this research also provides some suggestions how to systematically implement novel insights in daily clinical practice.

Most important deliverables of this thesis are:

1) a clearly defined clinical pathway, aimed at the moderately to severely affected arm-hand of subacute stroke patients, which may lead to a more efficient way of communication between clinicians and patients, as well as between clinicians and scientists with respect to how therapy content relates to the patients' goals, and to clearly describe and communicate the treatment steps that are actually taken during arm-hand therapy [2];

2) reference data, obtained from the single-armed prospective cohort study, describing how stroke patients, who were admitted to CARAS, actually perform during and after rehabilitation;

3) suggestions on how to incorporate and systematically investigate technological and/or pharmacological interventions within the existing CARAS treatment, which may facilitate patients and/or clinicians during arm-hand rehabilitation in improving patients' performance. In addition, the information on how technological and/or pharmaceutical interventions that are used in conjunction with the designed clinical care pathway can be assessed, may also be beneficial to other stakeholders like innovative companies and knowledge institutes. 


\section{Valorization potential towards clinical experts and scientists}

The multitude and complexity of information regarding the treatment of the patients' affected arm and hand in the post-stroke phase requires an efficient treatment policy. Facilitating knowledge exchange among clinical experts and between scientists is an important first step to take [3]. Here, four steps for further valorisation are proposed:

Step 1: Setting a reference frame

CARAS contains a well-defined form of therapy-as-usual, substantiated with outcome data at AHF and AHSP level [4, 5]. CARAS' transparent nature and related data may be used to, 1) strengthen insight into effects and changes at outcome level, process level and structure level, and 2) provide insight into complex arm-hand impairments and concomitant interventions. In summary, information obtained can be used as a frame of reference for clinicians and scientists to discuss clinical and scientific questions with respect to this field. Sharing knowledge and experiences may accelerate consensus on how to develop and/or improve knowledge, skills and outcomes with respect to arm-hand interventions that are currently offered in stroke rehabilitation practice. Experiences acquired in the stroke population may also be applicable in rehabilitation of patients who experience arm-hand problems due to other forms of central neurologic disorders.

Step 2: Combining expertise across disciplines

Strengthening the connection between care and research, and creating an on-going interaction between clinical experts and researchers is critical in order to facilitate the exchange of relevant knowledge with respect to the field [6]. To develop and adjust arm-hand interventions to the patient's individual needs, and to design the set-up of evaluation studies with respect to novel arm-hand interventions in moderately to severely affected patients is considered to be complex. This complexity reflects the importance and need for clinicians and (clinical) scientists to formalize exchange of knowledge and learning, which would necessitate the development of a (so-called) Specialty, focused on bringing together expertise on arm-hand rehabilitation in patients with a central nervous system disorder.

\section{Step 3: Education program}

Following chapter 2 of this thesis, in which CARAS has been described, an education and training program of arm-hand rehabilitation has been developed (in Dutch: “ Opleidingsplan arm-hand revalidatie na centraal neurologisch letsel" (unpublished)). The training program has primarily been built to support clinical experts in their clinical 
decision-making process in patients with different levels of arm-hand impairment after stroke. The main part of the training program contains 1) a module with respect to the analysis and observation of arm-hand skill performance, 2) a module containing the development of interventions in arm-hand skill performance training, and 3) a toolbox with supportive information, including various documents, such as the actual treatment program, a patient workbook, assignments and a manual.

Furthermore, throughout the Netherlands, the majority of the stroke survivors are discharged from the hospital to their home situation or are admitted to a nursing home [7]. Part of these patients also has to cope with a moderately to severely affected arm hand. Therefore, it is also relevant for clinical experts, who work in hospitals, nursing homes and/or home-based health-care centers to exchange and structure information with respect to the clinical management of the affected arm and hand in post-stroke phase. In fact, based on the complex nature of arm-hand rehabilitation, creating a network for arm-hand rehabilitation in central neurological disorders involving clinicians from a broad spectrum of rehabilitation care delivery organisations may optimize the quality of treatment, research and guidance of patients with an affected arm and hand. The description of CARAS and scientific output, as has been outlined in this thesis, may be used as supportive information in conjunction with the training course, thus valorizing the knowledge gathered in our research as presented in this thesis.

\section{Step 4: Consortium building}

Between 2014 and 2019 CARAS has been implemented in a number of stroke rehabilitation units in the Netherlands which now are united in a consortium (see Figure 1). The consortium provides a platform for discussion and learning methods with respect to topics related to arm-hand rehabilitation in post-stroke phase. Internationally, several stroke rehabilitation centers have implemented parts of CARAS. A step-wise implementation plan was developed in order to facilitate the implementation process of CARAS in other rehabilitation centers. An example of this implementation process is outlined in Figure 2. 


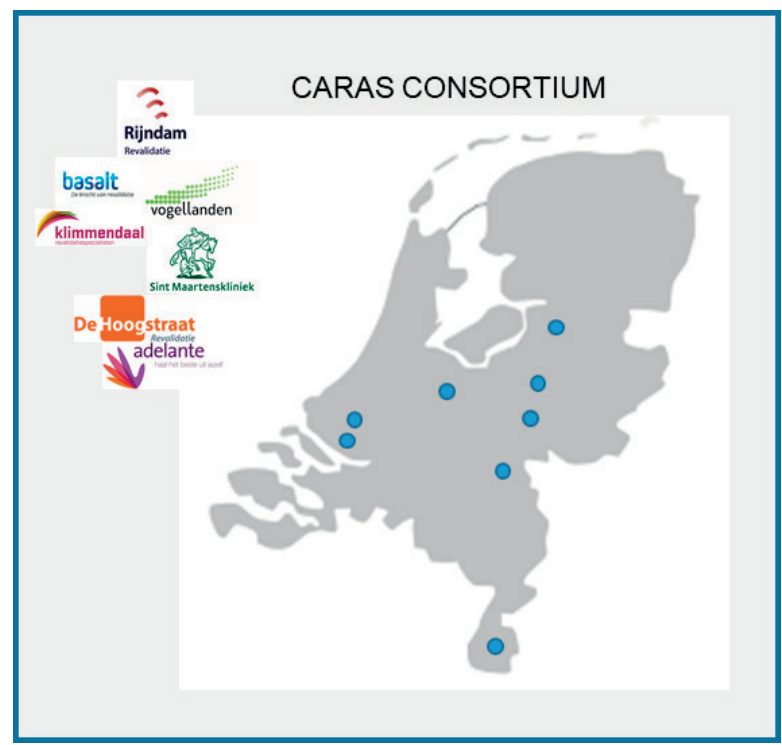

Figure 1. Rehabilitation centers in the Netherlands who joined the CARAS Consortium between 2014 and 2019

Organisation

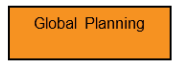
and arrangements

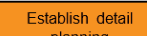

planning
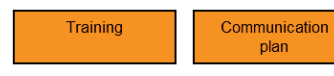

plan
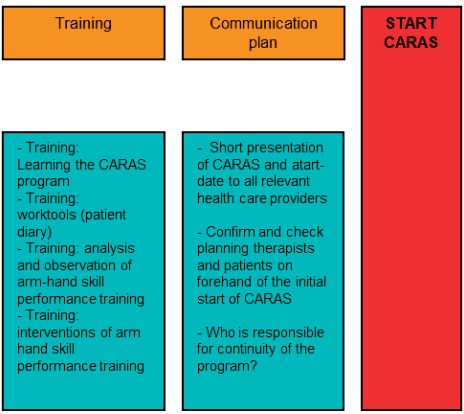

Controlling

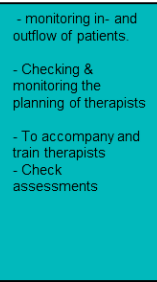

Figure 2. Example of an implementation schedule of CARAS. 


\section{Valorization regarding technological innovations}

The use of (rehabilitation) technology as part of regular treatment may be a solution in optimizing and improving treatment outcome in AHF and AHSP during rehabilitation $[8,9]$. From the perspective of manufacturers and distributors of technological and medical devices, there are several challenges with respect to the incorporation of technology in (daily) rehabilitation treatment. Many innovations do not mature and are not implemented in rehabilitation care. Also, impact assessments with respect to (cost) effectiveness often are missing and technology can be used erroneously.

To exchange knowledge and intensifying the collaboration between the development and manufacturing of technological devices with rehabilitation experts may lead to a more optimal use of a device and could even accelerate the approval process of a medical device.

A substantial part of technological devices, developed with the purpose to be used as a medical device are subject to a systematic clinical evaluation, according to the Medical Device Regulation (MDR) [10]. This procedure is required before the product is provided with a (CE) certificate. Only once the product is certified, it can be distributed to end-users, as for instance healthcare providers or patients. These regulatory requirements and obtaining the approval process, is likely to make the transition to stroke rehabilitation services a complicated and time-consuming process. Data with respect to the development of arm-hand function and arm-hand skill performance of patients who were allocated to CARAS can be used as a reference database and can be used as a route to (systematically) assess and evaluate new technological innovations with respect to arm-hand rehabilitation in post-stroke phase. An example of an international initiative in which the reference data of this thesis will be used for further (technological) developments is the European Interreg EMR project "Innovation and Implementation acceleration of Complex Rehabilitation Technology" (acronym: i2-CoRT) (https://www.i2-CoRT.eu/). For example, the CARAS program is being used in conjunction with a novel, 'remote handling concept'-based, task-oriented arm-hand skill performance training approach (ReHab-TOAT) for patients with a moderately to severely impaired arm-hand in the subacute and chronic stage after a stroke. Results from this combination of existing treatment (CARAS) and novel, technology-assisted training aspects may be used by companies developing new 
technologies in improving their product, bringing it in alignment with current training principles, thus valorising the (clinical) knowledge gathered.

\section{References}

1. Lai SM, Studenski S, Duncan PW, Perera S. Persisting consequences of stroke measured by the Stroke Impact Scale. Stroke; a journal of cerebral circulation. 2002;33(7):1840-4. PubMed PMID: 12105363.

2. Demetrios M, Brand C, Louie J, Khan F. More than a Black Box of Rehabilitation: Characterizing therapy programmes following Botulinum Toxin Injections for Spasticity in Adults with Stroke. Journal of Rehabilitation Medicine. 2016;48:426-34.

3. Oborn A, Barrett M, Racko G. Knowledge translation in healthcare: Incorporating theories of learning and knowledge from the management literature. Journal of Health Organisation and Management 2013;27(4):412 - 31. doi: 10.1108/JHOM-01-2012-0004.

4. Franck JA, Smeets RJEM, Seelen $H$. Changes in arm-hand function and arm-hand skill performance in patients after stroke during and after rehabilitation. PloS one 2017;12(6):1 18.

5. Franck JA, Smeets RJEM, Seelen HAM. Changes in actual arm-hand use in stroke patients during and after clinical rehabilitation involving a well-defined arm-hand rehabilitation program: A prospective cohort study. PloS one 2019:1-22.

doi: https://doi.org/10.1371/journal.pone.0214651.

6. Moore J, Shikako-Thomas K, Backus D. Knowledge Translation in Rehabilitation: A Shared Vision. Pediatric Physical Therapy 2017;29(3).

doi: 10.1097/PEP.0000000000000381. 
Summary 

Patients, suffering a stroke, rate improving their arm-hand function and incorporating their paretic hand in daily activities as a top priority in their rehabilitation. Nevertheless, even when stroke survivors experience some return of arm-hand function, it remains a common and significant source of long-term disability. For this reason, a wide range of different exercises or training methods, the use of equipment or techniques, and/or pharmacological interventions have been developed aimed at maximizing arm-hand recovery in order to improve functional ability and quality of life in post-stroke phase.

The set-up of most evaluation studies with respect to arm-hand interventions is protocol-based to serve a narrow band of well-described patients, and is less adaptable to accommodate patients with different levels of arm-hand impairment after stroke and/or patients with specific needs, as commonly seen in regular stroke rehabilitation centers.

Evidence-based interventions to improve and to maintain a sufficient level arm-hand function (AHF) and arm-hand skill performance (AHSP) in moderately or severely affected patients are scarce. Nevertheless, once associated with the prospect to dexterity, the opportunity to learn to use the full potential of their affected hand must be explored. In order to achieve this potential, patients have to be admitted to (intensive) arm-hand training interventions in the limited time window of maximum post-stroke neuroplasticity.

\section{CARAS}

The Concise Arm and hand Rehabilitation Approach in Stroke (CARAS) is designed for clinicians to structure and implement training of AHF and AHSP in stroke patients typically seen in every day clinical practice. Stratified based on the basis of their armhand severity level, i.e. mildly, moderately or severely impaired, patients are allocated to one of the three group-based training programs of CARAS. Severely affected patients are admitted to the program 'Taking care of the limb and prevention of complications' (program 1). Moderately affected patients were allocated to the 'Taskoriented gross motor grip performance program' (program 2), were they learn to use their affected hand to assist the non-paretic arm-hand during bimanual activities in daily life. Mildly affected patients admitted to the 'Functional AHSP training program' (program 3) participate in a task-oriented training program working immediately using more complex functional tasks. CARAS incorporates a stepwise clinical management 
procedure to elicit motor control problems and self-efficacy principles to assist patients to recognize and monitor improvements made in arm-hand performance. All three programs are well-documented and exist of time-delimited building blocks in order to systematically incorporate (new) technology and new evidence-based training elements swiftly.

\section{Changes in AHF and AHSP}

Once admitted to CARAS it was not well understood at what rate improvement (or deterioration) in AHF, AHSP and actual arm-hand use (as part of AHSP) occurred in sub-acute stroke patients with either a severely, moderately or mildly affected functional arm-hand, during and after their rehabilitation period. Nor was clear in which way AHF and AHSP differed between patients with a different levels of arm-hand severity.

The vast majority of patients admitted to CARAS' task-oriented training part, program 2 and program 3 respectively, improved considerably on AHF and AHSP. In addition, they were able to maintain these high levels during the post-rehabilitation phase. Largest gains with respect to AHF and AHSP occurred in moderately impaired patients. This means that also patients who lack any dexterity, i.e. finger extension at the start of program 2, are able to generate and maintain significant progressions in AHF and AHSP.

With respect to actual arm-hand-use it could be concluded that a vast majority of patients admitted to CARAS program 2 and program 3, improved on Intensity-of-use and Duration-of-use of their moderately or mildly affected arm-hand during rehabilitation. These results were also maintained across the post-rehabilitation phase. With respect to the ratio of Intensity-of-use between the affected and non-affected hand it can be stated that in mildly impaired patients the non-affected hand is used about one and a half times more than the affected hand in the post-rehabilitation period. Patients with a moderately affected hand use their non-affected hand about two and a half times more than their affected hand.

A minority of the severely affected patients, initially diagnosed with a severely impaired arm-hand, also improved considerably in terms of AHF and AHSP during and after the rehabilitation phase. Due to their progressions, these patients switched from program 1 to program 2, the task-oriented gross motor grip performance program in order to learn to use their affected arm-hand in daily performance tasks. 


\section{Interventions adjunct to CARAS}

In sub-acute stroke patients in who no or little dexterity in the initial phase of arm-hand rehabilitation was observed, arm-hand training led to improvements in AHF and AHSP. These improvements enlarged their possibilities to regain a sufficient level of dexterity in order to perform gross motor grip tasks and assist the non-paretic hand during simple bimanual activities in daily life.

In order to create an optimal retention of learning to use the full potential of the affected hand, patients' needs to push their limits by exploring actual arm-hand use. For this reason, patients have to work as independently as possible during therapy sessions, encouraged to use an efficient problem-solving strategy leading to an easier performance of a given task. To work actively and as independent as possible with the affected arm-hand in an (intensive) arm-hand skill training regime, these patients needed to be facilitated in generating sufficient voluntary force generation to execute a functional (gross motor) grasp.

In order to facilitate voluntary grasp-and-release movements in moderately to severely affected patients and to augment existing (intensive) therapies, the use and addedvalue of Abobotulinum toxin-A (ABoNt-A) and two non-actuated dynamic hand orthoses has been investigated. All three interventions were applied in conjunction with CARAS' gross motor grip performance program.

Besides a low motor ability level due to weakness, stroke survivors with a moderately to severely affected arm-hand may be hampered by post-stroke spasticity, which also may limit progressions in AHF and AHSP. A spasticity reduced treatment ABoNt-A was applied in the training phase in conjunction with CARAS' gross motor grip performance program, tailored to the patients' individual characteristics. At group level, the addedvalue of ABoNt-A on AHF and AHSP could not be confirmed. Results of the study showed that a minority of all study participants achieved significant additional improvements in arm-hand capacity. Half of the participants improved at the level of self-perceived performance. A majority of the participants demonstrated significant improvements in AHF. In a minority of patients, no beneficial effects from the contribution of ABoNt-A during arm-hand rehabilitation was observed. Patients who displayed improvements in the distal part of the arm before ABoNt-A was applied, may have obtained higher levels of arm-hand capacity, compared to the more severely 
affected subgroup. The application of botulinum toxin may have facilitated the reoccurrence of voluntary movements that were hampered by spasticity before.

In two separate studies, the added-value of two non-actuated dynamic hand orthoses, i.e. the Saeboglove, a tensioners-assisted hand orthosis, combined with electrical stimulation (ES) and the Saeboflex, a spring-loaded hand orthosis has been evaluated. The first study targeted moderately to severely sub-acute stroke patients who did not show any arm-hand dexterity prior to the intervention. Equipped with the orthosis and ES they were admitted to CARAS' program 2, gross motor grip performance program. Once they left the training phase, a vast majority of patients improved significantly on arm-hand capacity and maintained a certain level of arm-hand dexterity postrehabilitation.

In the second study, the majority of moderately to severely sub-acute stroke patients improved on arm-hand capacity. However, only half of the patients admitted to this study improved as to their self- perceived performance, relative to their baseline performance. Patients who showed little to no arm-hand improvements at an early stage post-stroke seemed to have benefitted more from the Saeboflex in conjunction with CARAS than those patients who showed clear improvements during the baseline phase. In both studies, patients' felt highly motivated to work with the orthosis in conjunction with a high-intensive task-oriented training regime. Also, the rated their sense of self-regulation as high.

\section{Conclusion}

Stroke patients with prospect regarding arm-hand recovery should be facilitated in creating an optimal retention in how to use the full potential of their affected hand outside therapeutic situations, i.e. in their own daily life pursuits as quickly as possible. CARAS has explicitly been designed to allow a heterogeneous group of stroke patients with a broad spectrum of arm-hand severity levels to participate in task-oriented practice, including patients with a moderately to severely affected arm-hand who display little or no dexterity the initial phase of rehabilitation. In the vast majority of mildly and moderately affected patients the training resulted in significantly improved AHF and AHSP levels, and possibly to meaningful daily arm-hand performance, also in post-rehabilitation. In addition, moderately to severely affected patients appeared to be facilitated by dynamic hand orthoses or botulinum toxin in conjunction with CARAS' 
gross motor program in order to provide functional grasp-and-release movements while reducing therapeutic assistance. 

Samenvatting 



\section{Samenvatting}

Revalidanten die, ten gevolge van een CVA, beperkingen ondervinden in een arm en hand beoordelen het verbeteren van de arm-handfunctie en het kunnen inzetten van de aangedane arm-hand in dagelijkse activiteiten als een topprioriteit.

Hoewel een deel van de revalidanten een bepaalde mate van herstel van de arm- en handfunctie ervaart, blijft de niet-inzetbare of beperkt inzetbare hand een belangrijke en frequent voorkomende oorzaak van handicap. Wereldwijd zijn inmiddels een aanzienlijke hoeveelheid trainingsmethoden en technologische en farmacologische interventies ontwikkeld die bedoeld zijn om het herstel van de aangedane arm-hand en de daarmee gepaard gaande kwaliteit van leven na een CVA te verbeteren.

De meeste evaluatiestudies met betrekking tot het beoordelen van de effectiviteit van arm-handinterventies zijn geprotocolleerd en gericht op een afgebakende populatie waarbij strikte inclusiecriteria worden gehanteerd. Dit bemoeilijkt de generalisatie van dergelijk trainingsprotocollen naar meer heterogene doelgroepen, zoals revalidanten met verschillende niveaus van arm-handfunctie en co-morbiditeit, frequent gezien in de reguliere revalidatie.

Evidence-based interventies, gericht op het verbeteren én het behouden van een voldoende niveau van arm-handfunctie en arm-handvaardigheid bij patiënten met een gemiddeld tot ernstig aangedane arm en hand, zijn schaars. Desalniettemin, eenmaal geassocieerd met het vooruitzicht tot enig herstel van de aangedane arm-hand, dienen ook revalidanten met een gemiddeld tot ernstig aangedane arm-hand de kans te krijgen om het volledig potentieel van de aangedane arm en hand te benutten. Door het toegankelijk maken van arm-hand trainingsinterventies voor deze specifieke doelgroep kan het volledig potentieel van arm-handfunctie en armhandvaardigheid eerder aangesproken worden, binnen de begrensde tijdsperiode waarin ook de plasticiteit van het brein optimaal benut kan worden. 


\section{CARAS}

De 'Concise Arm and hand Rehabilitation Approach in Stroke' (acroniem: CARAS) is ontwikkeld met als doel een brede doelgroep van revalidanten met een beperkte armhandfunctie ten gevolge van een CVA, optimaal te begeleiden tijdens het revalidatieproces. Binnen CARAS staat enerzijds het structureren van interventies, gericht op het behoud of het verbeteren van de aangedane arm-hand en anderzijds het implementeren van nieuwe trainingsmethodieken, die een bijdrage kunnen leveren aan het bespoedigen van het al dan niet partiële herstel van de arm-handfunctie en arm-handvaardigheid, centraal.

Eens verwezen naar CARAS, worden revalidanten, op basis van de ernst van de beperking van de aangedane arm en hand, gestratificeerd naar mild, matig of ernstig beperkte handfunctie. Vervolgens worden ze toegewezen aan één van de drie trainingsprogramma's in CARAS.

Patiënten met een ernstig aangedane arm-hand worden verwezen naar het programma waarin het verzorgen van de aangedane arm-hand en het voorkomen van complicaties centraal staat (programma 1). Revalidanten, gediagnosticeerd met een matig aangedane arm-hand worden verwezen naar programma 2, een taakgeoriënteerd training programma waarin het accent ligt op het leren van grofmotorische grijpvaardigheden. In programma 2 leren revalidanten de aangedane hand in te zetten als ondersteuning van de niet-aangedane arm-hand tijdens het uitvoeren van dagelijkse, veelal bimanuele, activiteiten. Revalidanten met een mild aangedane arm-hand doorlopen programma 3, een taak-georiënteerde training programma waarin geleerd wordt de aangedane arm-hand vrijwel direct in te zetten tijdens het uitvoeren van complexe functionele taken.

CARAS omvat een klinische procedure om de beperkte functionele inzetbaarheid van de aangedane arm-hand vast te stellen en vervolgens te trainen. Het structureel toepassen van self-efficacy principes ondersteunt patiënten tijdens de training in het tijdig (h)erkennen van de verbeterde functionele inzetbaarheid van de aangedane armhand. De programma's zijn duidelijk beschreven en bestaan uit in tijd afgebakende trainingsperiodes, hetgeen ook het implementeren van technologische, therapieondersteunende middelen en vernieuwende, evidence-based trainingselementen vereenvoudigt. 


\section{Veranderingen in arm-handfunctie en arm-handvaardigheid}

Nadat CARAS ontwikkeld was en toegepast werd in de kliniek ontstond de vraag in welke mate CARAS leidde tot verbetering (of achteruitgang) in arm-handfunctie, armhandvaardigheid en de daadwerkelijke inzet van de aangedane arm-hand (als onderdeel van arm-handvaardigheid).Tevens was het onduidelijk in welke mate een verandering van arm-handfunctie en arm-handvaardigheid plaatsvond bij revalidanten met een ernstig, matig of een mild aangedane arm-hand, in de sub-acute fase na CVA. Ook bleek niet duidelijk in welke mate en op welk moment deze veranderingen optraden en in welke mate de arm-handfunctie en arm-handvaardigheid verschilde tussen revalidanten met een ernstig, matig of mild aangedane arm-hand. Om op deze vragen antwoord te kunnen geven, is een grote prospectieve cohort studie opgezet.

De overgrote meerderheid van revalidanten die de taak-georiënteerde training van CARAS' programma 2 en programma 3 hebben doorlopen, vertoonde een significante verbetering ten aanzien van zowel arm-handfunctie als arm-handvaardigheid. De grote meerderheid van deze revalidanten behielden dit verbeterde niveau van armhandfunctie en arm-handvaardigheid tijdens de post-revalidatiefase. Meeste progressies in arm-handfunctie en arm-handvaardigheid werden geobserveerd bij revalidanten met een matig aangedane arm-hand. Dit betekende dat ook revalidanten die tijdens de aanvang van programma 2 géén inzetbare hand hadden toch significant konden verbeteren wat betreft arm-handfunctie en arm-handvaardigheid, leidend tot een inzetbare arm-hand.

Met betrekking tot de daadwerkelijk inzetbaarheid van de aangedane arm-hand tijdens de revalidatieperiode kan worden geconcludeerd dat het overgrote deel van de revalidanten die hebben deelgenomen aan programma 2 en programma 3 significant verbeterden met betrekking tot zowel de intensiteit als de duur van inzetbaarheid van de aangedane arm en hand. Deze resultaten bleven bestaan tijdens de postrevalidatiefase. Tevens bleek dat in de mild aangedane groep revalidanten de nietaangedane arm-hand anderhalf keer meer werd ingezet dan de niet-aangedane armhand. In revalidanten met een matig aangedane arm-hand bleek dat de nietaangedane arm-hand tweeënhalf keer meer werd ingezet vergeleken met de aangedane arm-hand. 
Van de revalidanten die in de initiële fase van de revalidatie gediagnosticeerd waren met een ernstig aangedane arm-hand, vertoonde een minderheid een aanzienlijke verbetering in arm-handfunctie en arm-handvaardigheid tijdens het revalidatieproces. Vanwege de doorgemaakte progressies verwisselden deze revalidanten programma 1 voor het intensieve taak-georiënteerde programma 2, met als doel de hand te leren inzetten tijdens de uitvoering van dagelijkse taken.

\section{Het toepassen van interventies, aanvullend op CARAS}

Bij een aantal revalidanten, waarbij in de initiële fase van de revalidatie nauwelijks tot geen handfunctie was vastgesteld, leidde deelname aan arm-hand training tot verbeteringen in zowel arm-handfunctie als arm-handvaardigheid. Deze opgedane verbeteringen leidden uiteindelijk tot de mogelijkheid om met de aangedane arm-hand grove motorische grijptaken uit te voeren, waardoor eenvoudige bimanuele activiteiten uitgevoerd konden worden.

In het taak-georiënteerde gedeelte van CARAS werden de revalidanten uitgedaagd zelf de inzetbaarheid van de aangedane arm-hand te exploreren binnen dagelijkse activiteiten met als doel een optimaal leereffect te creëren.

Tijdens de arm-hand therapiesessies leerden revalidanten zo onafhankelijk mogelijk te werken en werden ze aangemoedigd om een zo efficiënt mogelijke strategie te leren gebruiken, leidend tot een eenvoudige, comfortabele uitvoering van een bepaalde vaardigheid of activiteit.

Om revalidanten te stimuleren om de aangedane arm-hand in een (intensief) armhandvaardigheid training regime zo zelfstandig mogelijk in te zetten, dienden zij te worden gefaciliteerd in het genereren van voldoende handkracht om een functionele greep herhaaldelijk uit te kunnen voeren. De toegevoegde waarde van Abotulinum Toxine A (ABoNt-A) en twee niet-geactueerde dynamische handorthesen in het vereenvoudigen van zowel het grijpen als het loslaten door de matig tot ernstig aangedane arm-hand, is geëvalueerd. Deze drie interventies zijn toegepast in combinatie met programma 2 van CARAS.

Revalidanten met een gemiddeld tot ernstig aangedane arm-hand worden, naast het hebben van te weinig spierkracht, ook vaak belemmerd door een vorm van spasticiteit, hetgeen de progressie in arm-handfunctie of arm-handvaardigheid tijdens de arm- 
handtraining kan hinderen. Tijdens de trainingsfase is daarom, afgestemd op de specifieke eigenschappen van de revalidant, een spasticiteit regulerende behandeling, i.c. ABoNt-A, toegediend in combinatie met programma 2 van CARAS.

Op groepsniveau kon de toegevoegde waarde van ABoNt-A op arm-handfunctie en arm-handvaardigheid niet worden bevestigd. Een minderheid van alle revalidanten die aan deze studie deelnamen, toonden een significante verbetering in armhandcapaciteit. De helft van de revalidanten liet significante verbeteringen zien met betrekking tot de eigen perceptie van de mate van inzetbaarheid van de aangedane arm-hand tijdens de uitvoering van dagelijkse, veelal bimanuele activiteiten.

In de meerderheid van de revalidanten die deelnamen aan de studie werden significante verbeteringen met betrekking tot arm-handfunctie geobserveerd. Bij een minderheid van de revalidanten die deelnamen aan deze studie werden geen additionele effecten van $A B o N t-A$ op de arm-handfunctie waargenomen. Revalidanten waarbij enig herstel in het distale gedeelte van de arm was geobserveerd alvorens ABoNt-A werd toegepast, hebben, uitgaand van de verbeterde arm-handcapaciteit, mogelijkerwijs meer geprofiteerd van de toegevoegde waarde van ABoNt-A in vergelijking met de subgroep die nauwelijks tot geen herstel vertoonden bij aanvang van deze studie. Het toevoegen van ABoNt-A bovenop de gebruikelijke therapie, i.c. CARAS, lijkt een faciliterend effect te hebben gehad op het genereren van vrijwillige bewegingen in de aangedane arm-hand die voorheen werden verhinderd door de spasticiteit.

In twee onafhankelijke studies is de toegevoegde waarde van twee niet-geactueerde dynamische hand ortheses geëvalueerd.

De eerste studie was gericht op revalidanten in de sub-acute fase waarbij geen handfunctie was geobserveerd vóór aanvang van de arm-handtraining. Revalidanten hebben deelgenomen aan CARAS' programma 2, uitgerust met de Saeboglove orthese, een elastiek-ondersteunde handorthese, in combinatie met elektrostimulatie. In het merendeel van de deelnemers werd een significante verbetering ten aanzien van arm-handcapaciteit geobserveerd. Dit niveau werd behouden in de periode na de revalidatie.

Ook de tweede studie betrof revalidanten met een gemiddeld tot ernstig arm-hand in de sub-acute fase na CVA die, uitgerust met de Saeboflex, een veer-ondersteunde hand orthese, deelnamen aan programma 2 van CARAS. Het merendeel van deze 
groep ondervond een significante verbetering met betrekking tot arm-handcapaciteit. Echter bij slechts de helft van de deelnemers werd een significante verbetering waargenomen met betrekking tot hun eigen perceptie van de mate van inzetbaarheid van de aangedane arm-hand tijdens de uitvoering van dagelijkse, veelal bimanuele activiteiten. Revalidanten bij wie in een vroeg stadium na een beroerte weinig tot geen verbeteringen in de aangedane arm-hand was geobserveerd, leken meer baat gehad te hebben van de Saeboflex in combinatie met arm-handtraining dan revalidanten waar in de baselinefase wel duidelijke progressies in arm-handfunctie was waargenomen. In beide onderzoeken voelden revalidanten zich zeer gemotiveerd om met de orthese te werken in combinatie met het intensieve, taakgerichte trainingsprogramma. De mogelijkheid om de therapie naar eigen behoefte te kunnen aanpassen, gebaseerd op actuele en persoonlijke trainingsdoelen, werd door de deelnemers zeer positief beoordeeld.

\section{Conclusie}

Revalidanten die na het CVA enig vooruitzicht hebben op motorisch herstel van de aangedane arm-hand dienen te worden gefaciliteerd om het volledig potentieel van de aangedane arm-hand zo snel en zo optimaal mogelijk in te zetten tijdens de dagelijkse handelingen, buiten therapeutische situaties.

CARAS is expliciet ontworpen om taak-georiënteerde training toegankelijk te maken voor een brede doelgroep van revalidanten die, ten gevolge van een CVA, een beperkte arm-handfunctie ondervinden. Ook revalidanten die nauwelijks handfunctie of zelfs geen handfunctie hebben, kunnen deelnemen aan CARAS.

Bij het merendeel van de revalidanten met een mild tot matig aangedane arm-hand leidt de training tot een significante verbetering in zowel de arm-handfunctie als de arm-handvaardigheid. Ook leidt de training veelal tot een verbetering in het uitvoeren van betekenisvolle activiteiten in de fase na de revalidatie. Revalidanten met een matig tot ernstig aangedane arm-hand hebben in veel gevallen baat bij de ondersteuning door een dynamische hand-orthese of bij het toedienen van ABoNt-A ter vermindering van spasticiteit, in combinatie met programma 2 van CARAS. Deze combinatie faciliteert het zelfstandig kunnen grijpen en loslaten met de aangedane hand en leidt tevens tot een afname van de noodzaak voor therapeutische begeleiding. Het toepassen van de handortheses en ABoNt-A maakt taak-georiënteerde training 
toegankelijk voor doelgroepen waarvan in eerste instantie wordt gedacht dat deze niet zouden kunnen deelnemen aan taak-georiënteerde arm-hand training. 

Dankwoord 

Zowel uit de wetenschappelijke hoek als uit de revalidatiesector is er een behoorlijk aantal mensen betrokken geweest die mij door de jaren heen hebben geholpen om dit proefschrift succesvol af te ronden. Allereerst wil ik de mensen bedanken die gerevalideerd hebben op de afdeling CVA bij Adelante. In totaal hebben maar liefst 119 revalidanten meegewerkt aan dit proefschrift. Niet alleen tijdens hun revalidatietraject, maar zelfs ook tot een jaar daarna, namen zij de moeite om een aantal keer per jaar naar Adelante af te reizen.

Om in deze fase van het leven waarin je behoorlijk wat tegenslag moet verwerken, deel te nemen aan onderzoek is een teken van moed en doorzettingsvermogen.

Mijn promotoren Henk Seelen en Rob Smeets ben ik veel dank verschuldigd. Zij hebben in grote mate bijgedragen aan de inhoud van dit proefschrift. Henk, zonder jouw eindeloze geduld zou ik niet zo ver gekomen zijn. Dank voor je kritische houding en het stellen van de juiste vragen. Rob, je hebt me altijd heel goed ondersteund door me te helpen focussen wanneer dat nodig was en door mijn werk snel en van duidelijk commentaar te voorzien. Ook heb je me enorm geholpen met het werven van fondsen, zodat ik de laatste jaren door kon werken, zonder me daar heel druk over te hoeven maken. Yvan, ik wil jou graag bedanken voor de goede faciliteiten die het kenniscentrum mij heeft geboden. Jule en Leonie, dank jullie wel voor het regelen en uitvoeren van metingen en het bijhouden van het CRF in de laatste studie.

Een andere belangrijke inspiratiebron voor mij waren, en zijn nog altijd mijn collega's van Adelante. Jullie hebben allemaal op eigen wijze bijgedragen aan dit proefschrift. Een heel fijne bijdrage was het overnemen van metingen als ik verhinderd was. Milou, Inge, Renee, dank jullie wel hiervoor. Ondanks de werkdruk maakten jullie tijd om mij te ondersteunen. Ook ben ik veel collega's dankbaar voor de vele ideeën en uitspraken met betrekking tot de ontwikkeling van arm-hand revalidatie. Ze motiveerden tot nadenken en vaak uiteindelijk ook tot het (door-) ontwikkelen van interventies.

Jos en Will, jullie ervaring en tomeloze inzet voor deze groep revalidanten heeft mij vanaf het eerste uur enorm geïnspireerd. Jos, je hebt mij in die jaren meermaals geholpen in de zoektocht om het beste uit de revalidant en mijzelf te halen. Jij was het die mij in 2003 parkeerde in het voormalig IRV gebouw met een uitdraai van Pubmed met de zin: 'zoek eens uit of CIMT iets voor ons is'. Will, jouw respect voor deze doelgroep en jouw warme hart voor de revalidant was iets wat ik altijd veel van heb mogen leren. 
Paul, jouw kalmte, hulp en humor op de momenten dat ik weer eens te veel hooi op mijn vork nam hebben me echt geholpen. We kunnen inmiddels samen nog wel een boekje schrijven of weer eens een cursus geven. Boeiende onderwerpen met betrekking tot centraal neurologisch letsel zijn er genoeg. We kunnen we ook gewoon weer bier gaan drinken in een ver land. Jolanda, jij was het die in 2004 tegen mij zei; 'misschien moet iemand zich toeleggen op arm-hand training'. Dat idee heb ik toen met beide handen aangenomen. Huub, door alle jaren heen hebben we vaak samen revalidanten gezien. Het sparren met jou heeft mij geholpen om mijn eigen gedachten en acties beter af te stemmen op vragen en behoeftes vanuit het behandelteam. Dick, ook wij hebben samen in het verleden veel revalidanten samen begeleid. Jouw optimisme en jouw altijd positieve instelling is fantastisch.

Kim, je stelde vertrouwen in mij als collega, en je gaf me de ruimte daar waar het nodig was. Wip, een gesprek met jou, je positief kritische vragen, het geeft me nog steeds veel energie. Ik hoop dat we nog een hele tijd samen kunnen werken. Martine, je pragmatische kijk op de revalidatie, 'wat moet er geregeld worden', vond ik altijd heel verfrissend.

Peter, dank je wel voor je luisterend oor, de tijd die je nam om al filosoferend tegen de dagelijkse beslommeringen aan te kijken. Het gaf vaak een betere kijk op wat er nu toe doet, niet alleen in het werk, maar in het leven zelf. Karolien, pas in de laatste studie van dit proefschrift zijn we wat nauwer met elkaar gaan samenwerken. Ik hoop dat we nog jaren van onze samenwerking gebruik kunnen maken, zowel binnen de kliniek als binnen onderzoek.

Melanie, onze gesprekken over motorisch leren en hoe we dat praktisch het beste konden vormgeven binnen de klinische setting heb ik als heel waardevol beschouwd. Sonja, Margareth, Veronique, Jolanda, John, dank jullie wel, voor de ondersteuning, een grap en een glimlach en de snelle acties als ik weer eens iets snel geregeld moest hebben.

In het eerste uur van het consortium, opgericht in 2017, heb ik de gelegenheid gekregen om een aantal collega ergo- en fysiotherapeuten, eveneens werkzaam in de medisch specialistische revalidatie, beter te leren kennen. Eugenie, Martine, Cecile, Matthijs, Jolanda, Iris, Andreas, Lisaso, Ivanka, Yvonne, Paula, Sonja, Geert-Willem, Josta, Paula, Bart, Marlou, Simone, Anna, Elise, Emmy, Marike, Franny en Tilly. Ik wil jullie allemaal bedanken voor jullie positiviteit en het vertrouwen wat jullie in mij hebben gesteld de afgelopen jaren. Met het CARAS consortium hebben we de afgelopen jaren op een unieke 
manier van elkaars kennis en ervaring gebruik mogen maken. Ik ben er van overtuigd dat onze kijk op arm-hand revalidatie na CVA in deze tijd op positieve wijze is veranderd.

Tevens wil ik hierbij een aantal mensen bedanken die zowel vanuit een bedrijfstechnisch oogpunt, maar zeker ook als persoon betrokken zijn geweest bij de totstandkoming van dit proefschrift. Mary, Antoinette, Claudia (Ispen Pharmaceutica), ik dank jullie hartelijk voor alle ondersteuning de afgelopen drie jaar, jullie persoonlijke benadering en de fijne samenwerking. Freek, (Hankamp Revalidatie), dank je wel voor je snelle acties, je oprechte en duidelijke mening over therapie en de toepassing van technische middelen. Ook dank ik je voor je vertrouwen in mij. Ik hoop dat we in de toekomst nog een tijd samen mogen werken aan een aantal projecten. Gerrit, Desiree en Leon (Schwa-medico Nederland), dank jullie wel voor de hele fijne samenwerking de afgelopen jaren. Henry, (Saebo US) thanks for all your efforts, product delivery, our discussions and your hospitality in Charleston.

Mam, pap, ik kan jullie helaas niet meer in de ogen kijken en het jullie zeggen, maar bedankt. Dank je wel dat ik van jullie een hoeveelheid creativiteit en doorzettingsvermogen heb meegekregen. Dat is me de afgelopen jaren goed van pas gekomen. Jongens, dank jullie wel voor alle afleiding, stomme grappen, het bier, slechte én goede jaren '90-'00 muziek. We zijn er altijd voor elkaar, en jullie staan altijd voor me klaar. Anne-Claire, het laatst jaar was geen eenvoudig jaar voor mij. Dank je wel voor je liefde, je steun, geduld en je positieve kijk op het leven, waar ik (nog steeds) veel van kan leren. Florien en Elise, we hebben nu nog meer tijd om samen te stoeien, te knutselen, te koken, muziek te maken, muziek te luisteren of te sporten $($ ).

Dank jullie wel allemaal,

Han 

About the author 



\section{Biography}

Johan Anton (Han) Franck was born in Maastricht, The Netherlands in 1976.

In 2000 he received his bachelor degree as an occupational therapist at Zuyd University of Applied Sciences in Heerlen. From 2002 onwards he has been working as a therapist in neurological rehabilitation in adolescents and adults at Adelante Rehabilitation Centre in Hoensbroek. Over the years he developed a particular interest in the clinical management of arm-hand problems in the rehabilitation of patients who have suffered a stroke, with a special interest in stroke survivors suffering from a moderately to severely impaired arm and hand. In 2010 he received his master degree in Public Health, with a specialization in Health Services Innovation, at Maastricht University. In 2013 he started as a part-time PhD student at the department of Rehabilitation Medicine of the Research School CAPHRI at Maastricht University, Maastricht, the Netherlands.

Han Franck lives together with Anne-Claire Schrijnemaekers and his two children, Florien en Elise.

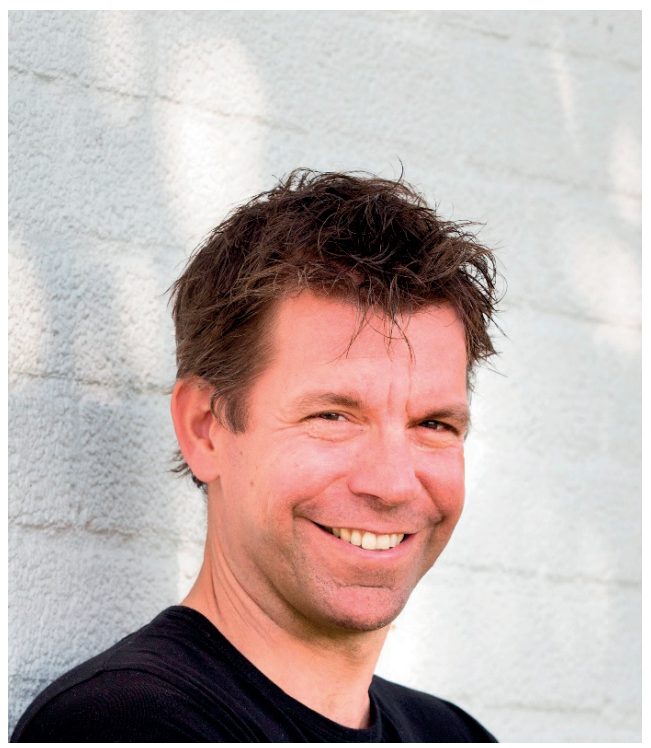





\section{List of publications}





\section{Publications}

Concise Arm and hand Rehabilitation Approach in Stroke (CARAS): A practical and evidencebased framework for clinical rehabilitation management. Johan A. Franck, Jos H.G. Halfens, Rob J.E.M. Smeets, Henk A.M. Seelen. Open Journal of Occupational Therapy, Issue 10, 2015.

Changes in arm-hand function and arm-hand skill performance in patients after stroke during and after rehabilitation. Reference data on 'therapy-as-usual'. Franck JA, Smeets RJEM, Seelen HAM. PLoS ONE 12(6) June 2017.doi.org/10.1371/journal.pone.0179453.

Changes in actual arm-hand use in stroke patients during and after clinical rehabilitation involving a well-defined arm-hand rehabilitation program: A prospective cohort study.

Franck JA, Smeets RJEM, Seelen HAM PLoS ONE 14 (4) April 2019. doi.org/10.1371/journal.pone.0214651.

Added-value of early post-stroke spasticity reduction during arm-hand rehabilitation in improving functional arm-hand skill performance: A multiple baseline single case experimental design study.

Franck JA, Smeets RJEM, Seelen HAM. International Journal of Neurorehabilitation 2018, 5:4. doi:10.4172/2376-0281.1000323.

Evaluation of a functional hand orthosis combined with electrical stimulation adjunct to armhand rehabilitation in subacute stroke patients with a severely to moderately affected hand function. Franck JA, Smeets RJEM, Seelen HAM. Disability and Rehabilitation.Jan 9: 1-9. doi.org/10.1080/09638288.2017.1423400.

Effects of a dynamic hand orthosis for functional use of the impaired upper limb in sub-acute stroke patients: a multiple single case experimental design study. Johan A. Franck, Annick A.A. Timmermans, Henk A.M. Seelen; Technology and Disability 25 (2013) 177-187. 
LIST OF PUBLICATIONS

\section{Submitted publication}

Added-value of spasticity reduction to improve arm-hand skill performance in sub-acute stroke patients with a moderately to severely affected arm-hand. Franck JA, Smeets RJEM, Seelen HAM. 

